\title{
Single-Chip Scanning Probe Microscopes
}

by

\section{Niladri Sarkar}

\author{
A thesis \\ presented to the University of Waterloo \\ in fulfillment of the \\ thesis requirement for the degree of \\ Doctor of Philosophy \\ in \\ Electrical and Computer Engineering
}

Waterloo, Ontario, Canada, 2013

(c) Niladri Sarkar 2013 


\section{AUTHOR'S DECLARATION}

I hereby declare that I am the sole author of this thesis. 


\begin{abstract}
Scanning probe microscopes (SPMs) are the highest resolution imaging instruments available today and are among the most important tools in nanoscience. Conventional SPMs suffer from several drawbacks owing to their large and bulky construction and to the use of piezoelectric materials. Large scanners have low resonant frequencies that limit their achievable imaging bandwidth and render them susceptible to disturbance from ambient vibrations. Array approaches have been used to alleviate the bandwidth bottleneck; however as arrays are scaled upwards, the scanning speed must decline to accommodate larger payloads. In addition, the long mechanical path from the tip to the sample contributes thermal drift. Furthermore, intrinsic properties of piezoelectric materials result in creep and hysteresis, which contribute to image distortion. The tip-sample interaction signals are often measured with optical configurations that require large free-space paths, are cumbersome to align, and add to the high cost of state-of-the-art SPM systems. These shortcomings have stifled the widespread adoption of SPMs by the nanometrology community. Tiny, inexpensive, fast, stable and independent SPMs that do not incur bandwidth penalties upon array scaling would therefore be most welcome.

The present research demonstrates, for the first time, that all of the mechanical and electrical components that are required for the SPM to capture an image can be scaled and integrated onto a single CMOS chip. Principles of microsystem design are applied to produce single-chip instruments that acquire images of underlying samples on their own, without the need for off-chip scanners or sensors. Furthermore, it is shown that the instruments enjoy a multitude of performance benefits that stem from CMOS-MEMS integration and volumetric scaling of scanners by a factor of 1 million.
\end{abstract}

This dissertation details the design, fabrication and imaging results of the first single-chip contact-mode AFMs, with integrated piezoresistive strain sensing cantilevers and scanning in three degrees-offreedom (DOFs). Static AFMs and quasi-static AFMs are both reported.

This work also includes the development, fabrication and imaging results of the first single-chip dynamic AFMs, with integrated flexural resonant cantilevers and 3 DOF scanning. Single-chip Amplitude Modulation AFMs (AM-AFMs) and Frequency Modulation AFMs (FM-AFMs) are both shown to be capable of imaging samples without the need for any off-chip sensors or actuators.

A method to increase the quality factor ( $Q$-factor) of flexural resonators is introduced. The method relies on an internal energy pumping mechanism that is based on the interplay between electrical, 
mechanical, and thermal effects. To the best of the author's knowledge, the devices that are designed to harness these effects possess the highest electromechanical Qs reported for flexural resonators operating in air; electrically measured $Q$ is enhanced from $\sim 50$ to $\sim 50,000$ in one exemplary device. A physical explanation for the underlying mechanism is proposed.

The design, fabrication, imaging, and tip-based lithographic patterning with the first single-chip Scanning Thermal Microscopes (SThMs) are also presented. In addition to 3 DOF scanning, these devices possess integrated, thermally isolated temperature sensors to detect heat transfer in the tip-sample region. Imaging is reported with thermocouple-based devices and patterning is reported with resistive heater/sensors.

An "isothermal electrothermal scanner" is designed and fabricated, and a method to operate it is detailed. The mechanism, based on electrothermal actuation, maintains a constant temperature in a central location while positioning a payload over a range of $>35 \mu \mathrm{m}$, thereby suppressing the deleterious thermal crosstalk effects that have thus far plagued thermally actuated devices with integrated sensors.

In the thesis, models are developed to guide the design of single-chip SPMs and to provide an interpretation of experimental results. The modelling efforts include lumped element model development for each component of single-chip SPMs in the electrical, thermal and mechanical domains. In addition, noise models are developed for various components of the instruments, including temperature-based position sensors, piezoresistive cantilevers, and digitally controlled positioning devices. 


\section{Acknowledgements}

I have only the fondest memories of graduate school, and for this I owe a debt of gratitude to some exceptional people.

I have been most fortunate to have Dr. Raafat Mansour as my advisor. Over the years I have learned that he is a man with many carrots, but no sticks. He provided access to state-of-the-art resources, gentle guidance, and conveyed a passion for engineering and discovery, all while being a role model for integrity.

The seed of this work was planted while I was a co-op student at Zyvex. Jim Von Ehr had created a unique situation there by assembling a team of scientists of the highest caliber, and offering them seemingly unlimited resources. Instead of thinking of "the intern" as a liability, these scientists took me under their wing. I was encouraged to let my curiosity run wild and to regard the unknown with a sense of adventure. I thank George Skidmore for teaching me the importance of being incisive when formulating and answering research questions, and for his friendship; Christof Baur for telling me to hold my breath while delicate measurements were in progress, and for countless miles in the Cessna; Mark Dyer for sharing his intricate knowledge of instrumentation; John Randall and Jim for supporting my research throughout the years as an employee and a consultant; and my colleagues Jeff, Zoran, Matt, Ken, Taylor, Mike, and Aaron for the teamwork and the good times. Quitting my job in order to pursue a PhD was certainly a difficult decision.

I learned much of what I know about CMOS-MEMS at Carnegie Mellon University under the guidance of Gary Fedder, an outstanding contributor to this field. Peter Gilgunn set an example of academic rigor, and was a pleasure to work with. Mike Abdel-Malek, Sarah Bedair and Zoran Jandric made my time in Pittsburgh enjoyable by experiencing the finer things in life with me.

At the CIRFE lab, I was fortunate to work with great mentors and colleagues. For their contributions and for creating a stimulating and enjoyable environment, I thank Bill Jolley, Kyle Trainor, Nino Zahirovic, Geoffrey Lee, Duncan Strathearn, Mahdi Olfat, Winter Yan and Siamak Fouladi. For the extra-curriculars, I thank Cmac, Kev, Pepi, Rohan, and Lynsi.

I appreciate the time and effort of my committee members Drs. Eihab Abdel-Rahman, Robert Gorbet, and Siva Sivoththaman; thank you for reading this dissertation and providing valuable feedback. I am particularly grateful to Professor Roger Howe for agreeing to be the external examiner, reading the thesis, and travelling to attend my defense. 
I am truly indebted to Lynsi, who did more than her share around the house and provided sustenance and motivation while I sat, atrophying at the computer.

This work was supported by the Ontario Research Fund - Research Excellence program (ORF-RE), the Defense Advanced Research Projects Agency (DARPA), and Integrated Circuit Scanning Probe Instruments (ICSPI Corp). I was fortunate to receive scholarships from the Natural Science and Engineering Research Council of Canada (NSERC), the Ontario Graduate Scholarship (OGS) Program, and the Waterloo Institute for Nanotechnology (WIN).

Finally, I wish to thank my family. Throughout my formative years, they nurtured my curiosity and fostered a passion for learning. My mother Gopa, father Nimai and sister Nindi have been an unwavering source of support, love and encouragement. 


\section{Dedication}

To Ma and Baba, you have always put my education ahead of yourselves. 


\section{Table of Contents}

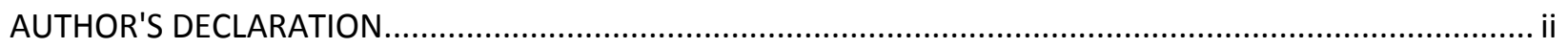

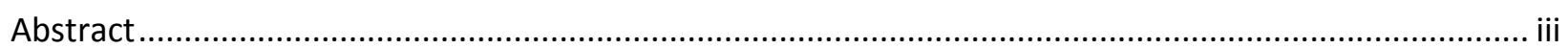

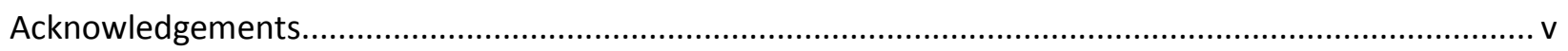

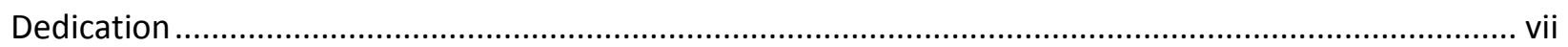

List of Figures

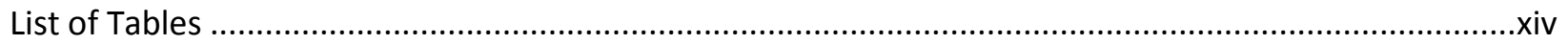

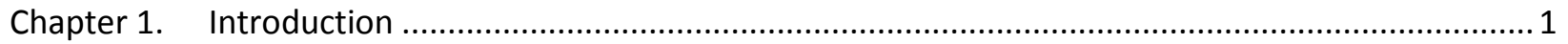

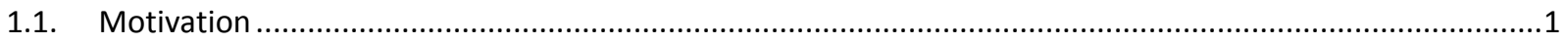

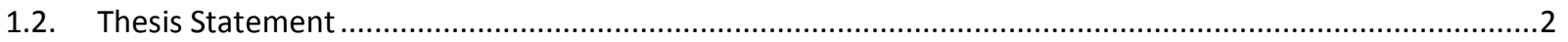

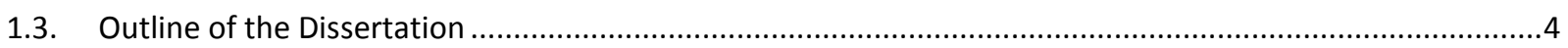

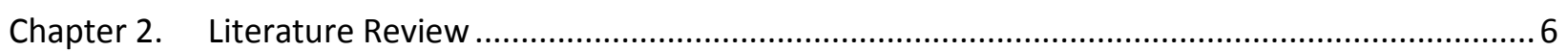

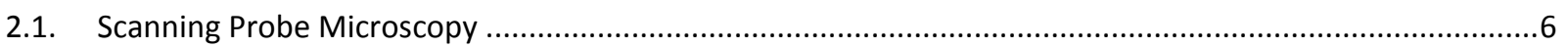

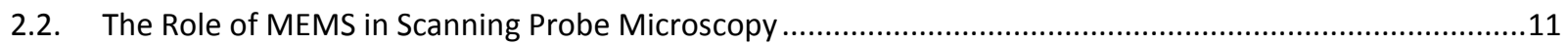

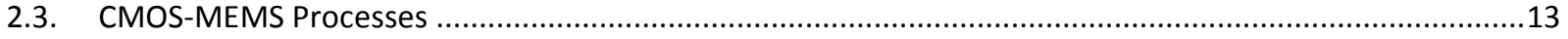

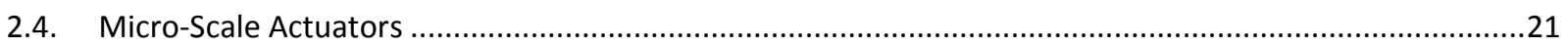

Chapter 3. Development of Coupled Electrothermomechanical Models...............................................2

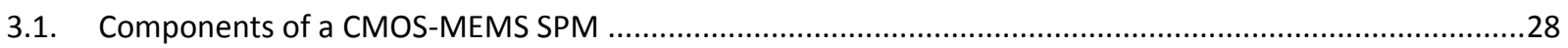

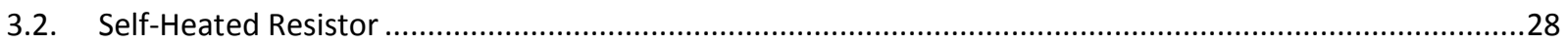

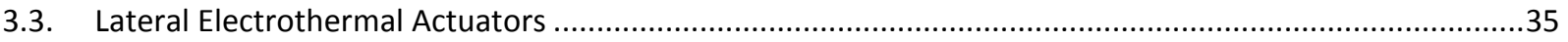

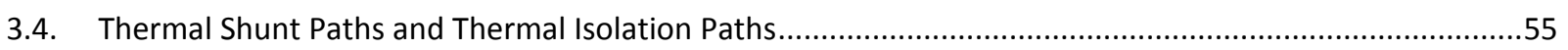

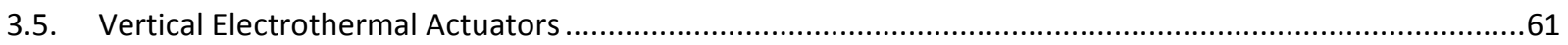

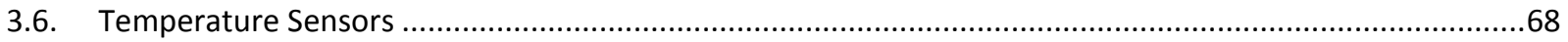

Chapter 4. Quality Factor Enhancement in Resonant Cantilevers with Integrated Piezoresistors ..... 73

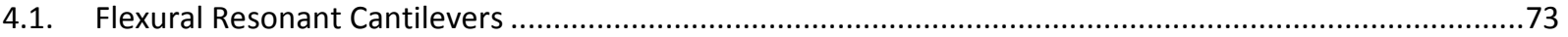

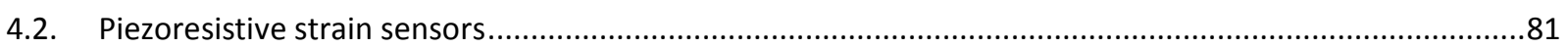

4.3. Electromechanical and Mechanical Quality Factor Enhancement .......................................................99

Chapter 5. Drive-Sense Strategies in the Presence of Noise .......................................................... 119

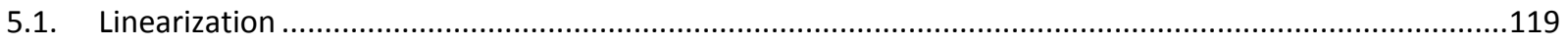

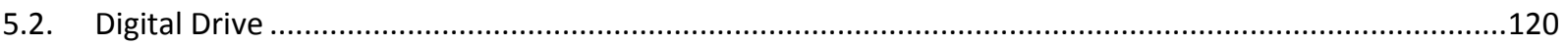

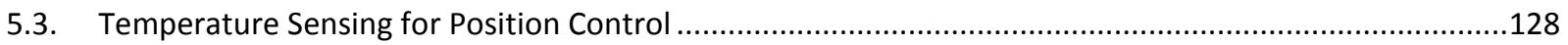

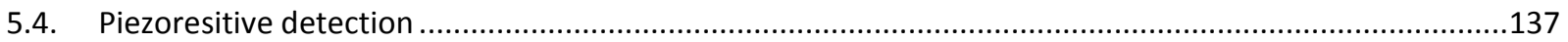

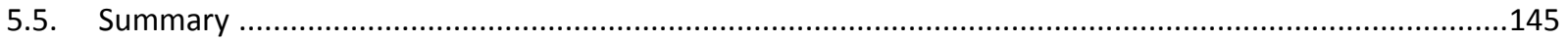

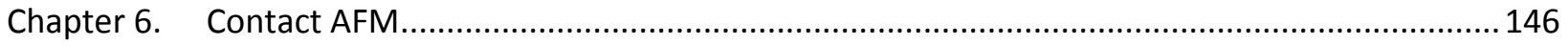

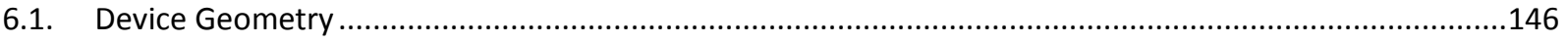

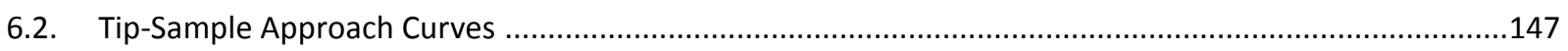




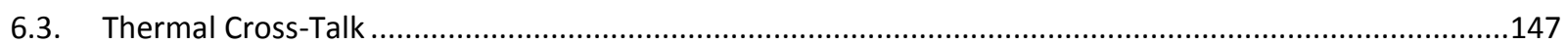

6.4. Forced Oscillation and Higher Harmonic Detection ...................................................................150

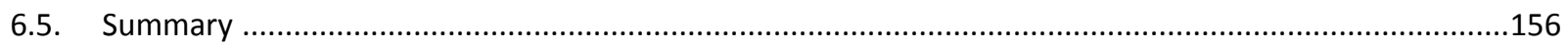

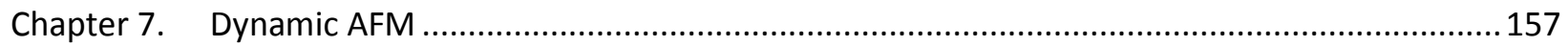

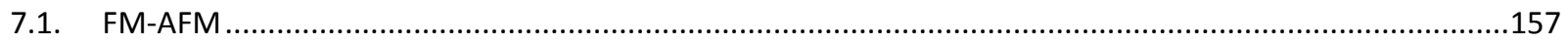

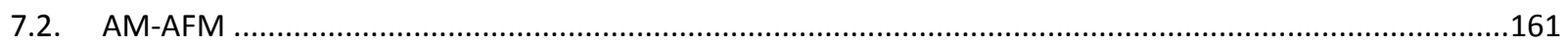

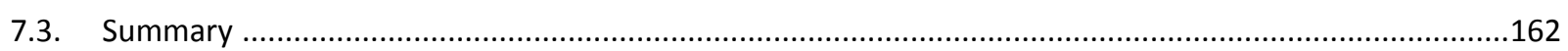

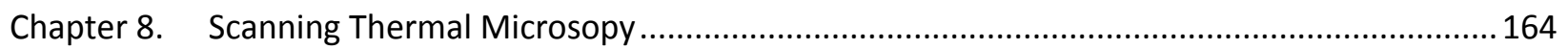

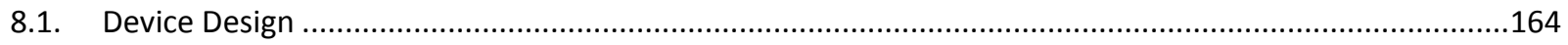

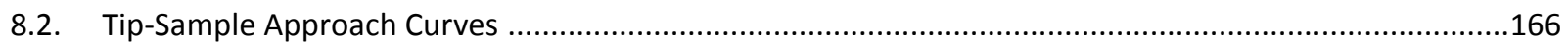

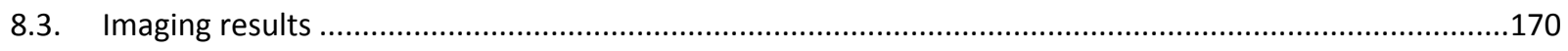

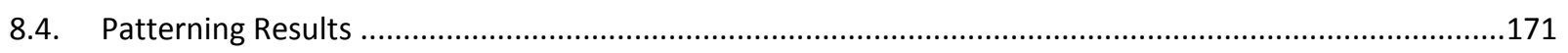

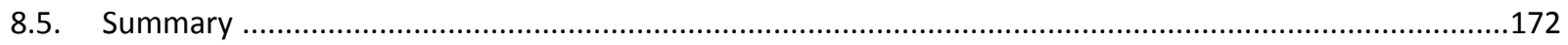

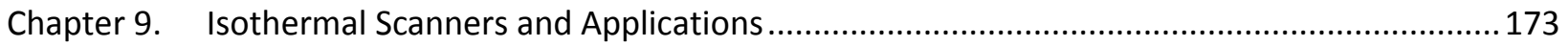

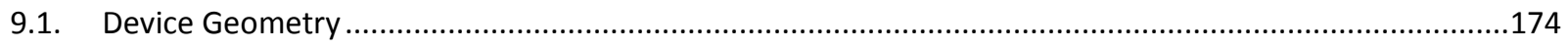

9.2. Isothermal Scanner Parasitic Effects and Mitigation....................................................................177

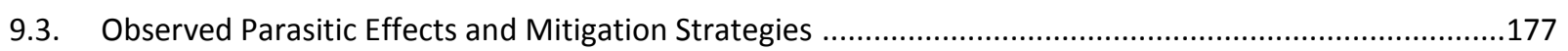

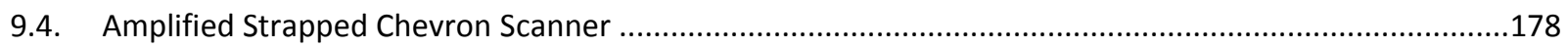

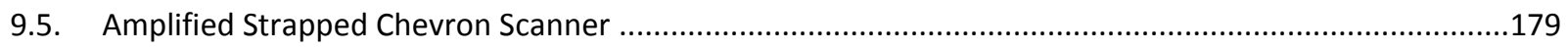

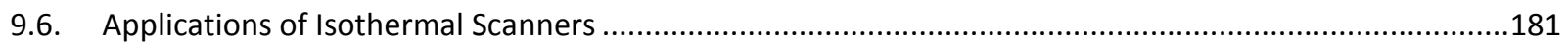

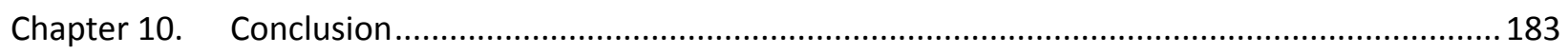

Appendix A MEMS Design Philosophy and Scaling Laws ...................................................... 186

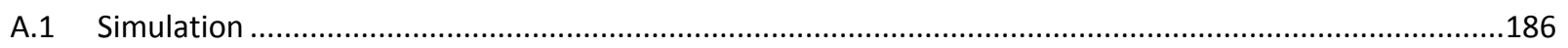

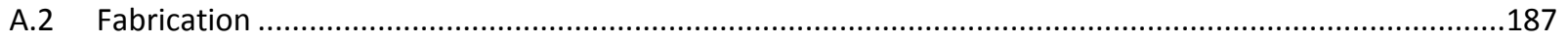

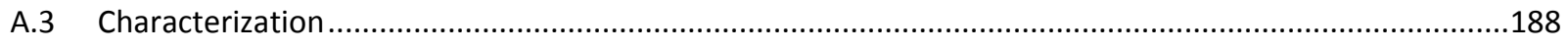

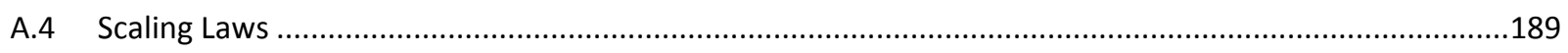

Appendix B Modeling of Electrothermal Actuators ............................................................ 195

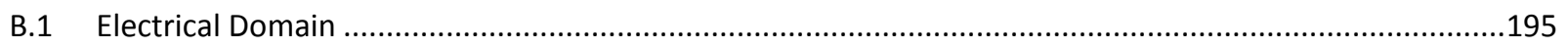

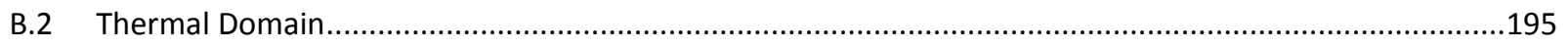

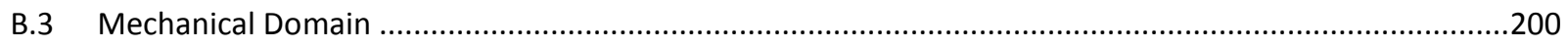

Appendix C Derivation of Lumped Parameter Component Values ...............................................207

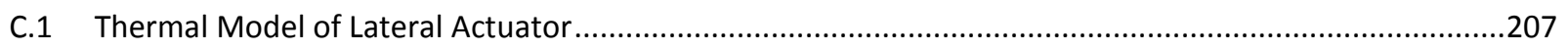

C.2 Mechanical Model of Lateral Electrothermal Actuators ................................................................211

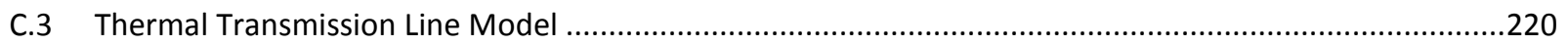

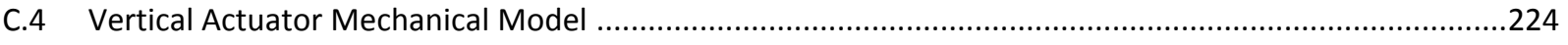

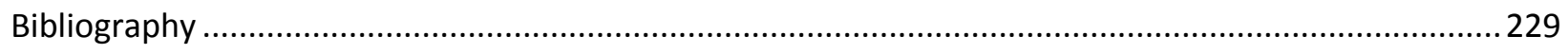




\section{List of Figures}

Figure 1: The ASIMPs (Application Specific Integrated MEMS Processes) CMOS-MEMS process results in released microstructures suspended over a cavity in the substrate. b) An accelerometer fabricated with

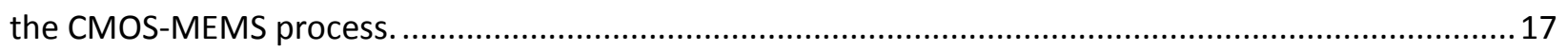

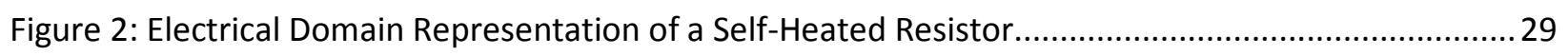

Figure 3: Thermal Domain Representation of a Self-Heated Resistor .................................................... 30

Figure 4: FEA Simulation of Bimorph Under Base (left) and Tip (right) heating ..................................... 31

Figure 5: Electro-Thermal Representation of a Self-Heated Resistor ..................................................... 32

Figure 6: The temperature coefficients of resistivity in CMOS polysilicon are strain-dependent...............34

Figure 7: Lateral Multimorph Geometry. The cross-section of the beam contains lower metal layers that are laterally offset from the top metal layers in order to create a bending moment. Beams are folded to

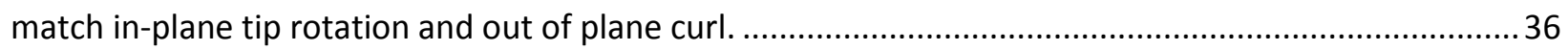

Figure 8: Thermal Domain Representation of a Lateral Electrothermal Actuator ................................... 40

Figure 9: Mechanical Domain Representation of a Lateral Electrothermal Actuator ................................ 40

Figure 10: Mechanical Domain Representation of Electrothermal Actuator Deflection........................... 42

Figure 11: Electro-Thermo-Mechanical Model of Deflection .............................................................. 43

Figure 12: Deflection of Thermally Isolated Bimorphs in Vacuum. (a) Test structure before actuation. (b) Test structure under an applied square wave voltage. Inset: Test structure after thermally induced plastic deformation.

Figure 13: Frequency Response (left) and Static Deflection (right) of Thermally Isolated Bimorphs in Air45 Figure 14: Scan Linearization with Square-Root Circuit. SEM image corresponds to a slow scan in the direction orthogonal to actuation. .46

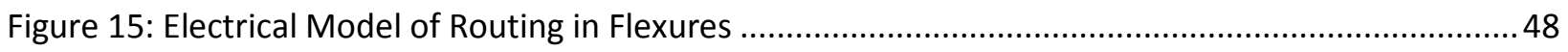

Figure 16: Mechanical Model of X-Y Flexural Suspension .................................................................. 50

Figure 17: Flexural Suspension for Cylindrical Coordinate Scanning ......................................................51

Figure 18: FEA of Cylindrical Scanner Flexural Suspensions. Top Left: S-shaped springs are compliant in the Z-direction. Center: Torsional spring is compliant due to angled segments. Right: Improved

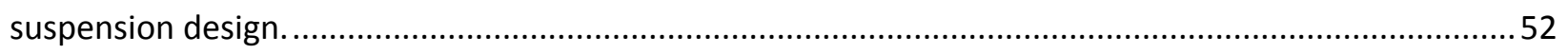

Figure 19: Mechanical Model of Cylindrical Scanner Flexural Suspension .............................................5 53

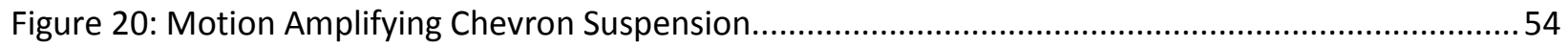

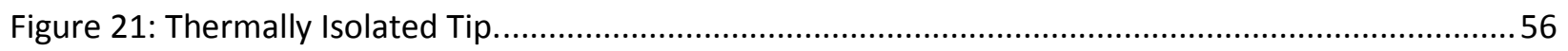




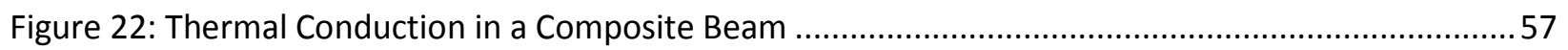

Figure 23: Thermal Domain Model of a Thermal Isolation Chain .........................................................58

Figure 24: Amplitude and Phase Dependence of Temperature in a Thermal Isolation Chain ...................59

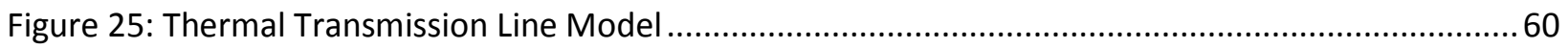

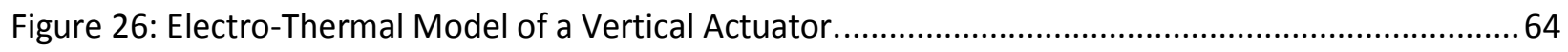

Figure 27: Transient Simulation of a Vertical Actuator at Different Input Frequencies...........................65

Figure 28: Electro-Thermo-Mechanical Model of a Vertical Actuator...................................................67

Figure 29: Resistive Temperature Sensor (Left) and Thermocouple (Right) ........................................69

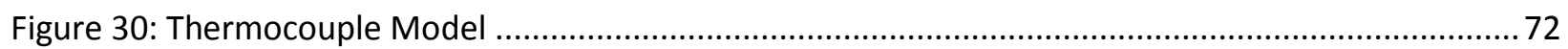

Figure 31: Externally Actuated Resonant Cantilevers .......................................................................... 76

Figure 32: Thermal Model of Externally Actuated Resonant Cantilever ................................................... 78

Figure 33: Thermal Domain Model of Externally Actuated Resonant Cantilever ..................................... 79

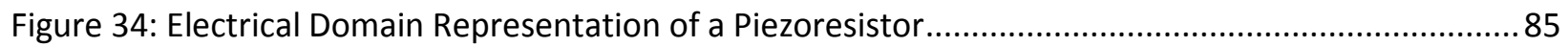

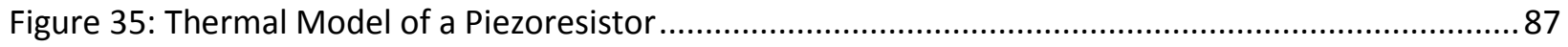

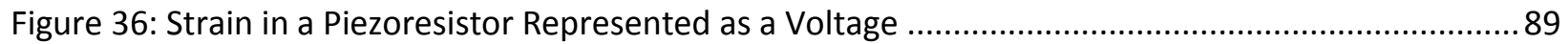

Figure 37: First Mode Shape and its Derivatives for a Resonant Cantilever........................................... 90

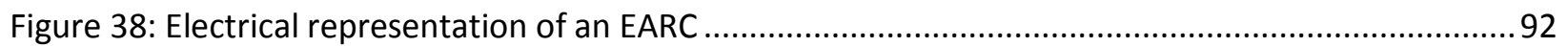

Figure 39: Loading Effect of Vertical Actuator on EARC Frequency Response .......................................93

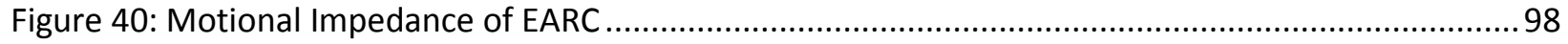

Figure 41: Modulation of the AC Amplitude of Vibration by a DC offset. ...............................................99

Figure 42: Hybrid Mode of Operation That Enhances Quality Factor .................................................100

Figure 43: Configuration of piezoresistive bridge circuit used for dynamic operation of cantilevers......101

Figure 44: Measurements of hybrid-mode operation reflect quantities in the electrical, thermal and

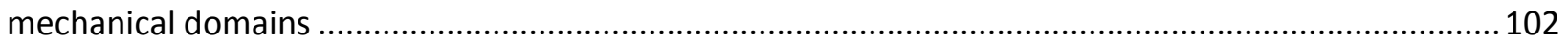

Figure 45: Temperature, strain and resistance change in piezoresistor (simulated) ............................ 104

Figure 46: Simulated Bode plots of sensor input, balance input, and differential measurement. ...........104

Figure 47: Comparison of simulations to data obtained by tuning the DC bias applied to the bridge .... 105

Figure 48: Quality Factor Enhancement as a Function of DC Bias Voltage in a Self-Actuated Resonant

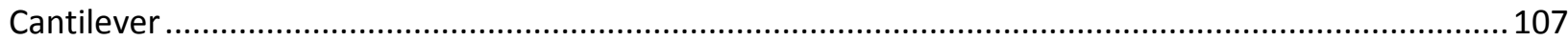

Figure 48: Quality Factor Enhancement Under Driving Force Control ................................................... 111

Figure 49: Thermal Runaway in the SARC Model. Time domain representation of the temperature in a resistor experiencing self-heating, with various values for the TCR ................................................. 116 
Figure 51: Left, linear response of electrothermal actuator to a PWM signal. Right, step response obtained in open-loop, with an analog PID controller, with closed-loop PWM, and with closed-loop Sigma-Delta drive. 121

Figure 52: Electronic R-2R DAC (a) and MEMS implementation (c). SEM image shows beams of compliance $\mathrm{C}$ and $2 \mathrm{C}$ in the network. A latch is present for power-off positioning at the output, and the LSB and MSB are indicated. The piezoresistive strain gauge is located within the load beam near the LSB. Hard stops are used to limit the input position to 2 distinct values (b) ..............................................122

Figure 53: Location of Piezoresistors to Measure Lateral Position in LSB ...........................................123

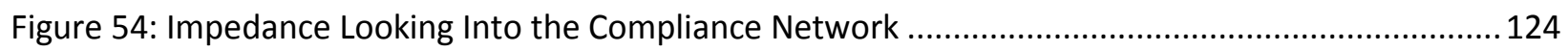

Figure 55: Finite Element Model, Lumped Element Model and measurement results. Significant DNL

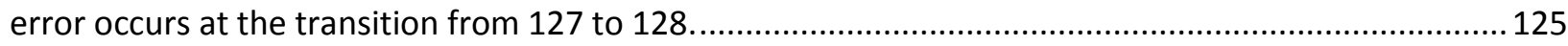

Figure 56: Coventor simulation of the compliance network in a state corresponding to input [01111110] (a). The MSB is the right-most bit connected to the output shuttle, and the LSB is the left-most bit. A 2D MEMDAC-based Scanner (b). The concept of performing a fine scan using the LSB in a grid defined by

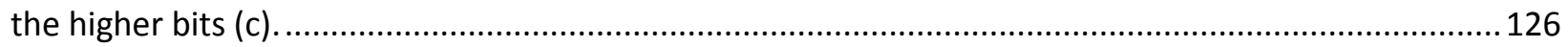

Figure 57: Noise Sources in the Wheatstone Bridge Circuit ............................................................ 130

Figure 58: Signal-to-Noise Ratio vs. Input Voltage in Temperature-Based Position Sensing ...................133

Figure 59: Magnified Plot of Signal-to-Noise Ratio vs. Input Voltage in Temperature-based Position

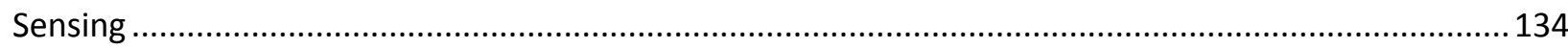

Figure 60: TCR-Based Position Sensing in the Vertical Actuator ....................................................... 135

Figure 61: PID Control of the Vertical Actuator in the Presence of Thermal Coupling............................ 136

Figure 62: Minimum Detectable Force for Various Piezoresistor Lengths .............................................139

Figure 63: Signal-to-Noise Ratio for Short Piezoresistors .................................................................. 140

Figure 64: Signal-to-Noise Ratio for Short Piezoresistors ….............................................................. 140

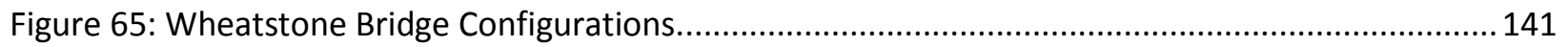

Figure 66: Placement of Balanced Piezoresistors in a Contact AFM Device........................................... 142

Figure 67: Cantilever Output Under Varying Contact Conditions........................................................ 143

Figure 68: Single-Chip Contact AFM Device Geometry.................................................................. 146

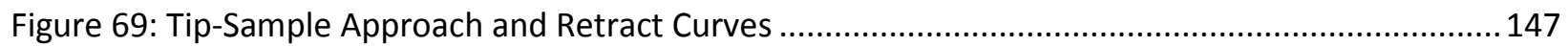

Figure 70: Measurement of Parasitic Coupling Between Lateral Actuators.......................................... 148

Figure 71: Temperature Coefficient of Resistivity-based Control of a Vertical Bimorph ........................ 149

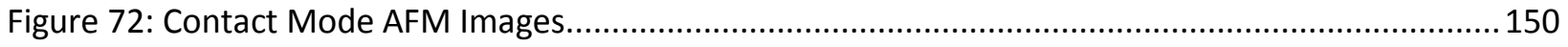


Figure 73: Data Obtained While the AFM is in Intermittent Contact with a Sample 151

Figure 74: Higher Harmonic Detection Concept

Figure 75: Packaging and Test Setup. a) released die after singulation, b) PCB carrier, c) packaged and wirebonded SPMs, d) Test Setup 153

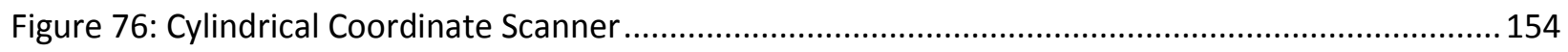

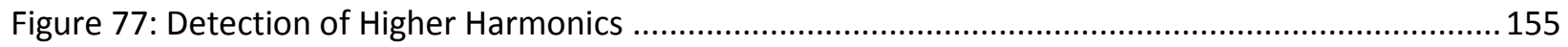

Figure 78: Images Captured at the Third Harmonic.......................................................................... 155

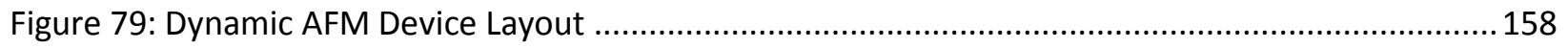

Figure 80: Close-up of Dynamic AFM Cantilever and Balanced Piezoresistors .....................................158

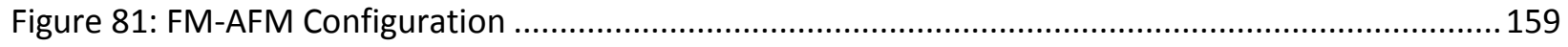

Figure 82: Q-Factor and Resonant Frequency of Dynamic AFM as a Function of Pressure ....................160

Figure 83: FM-AFM Images of a calibration grating (left) and Exposed Vias on a CMOS-MEMS chip......161

Figure 84: AM-AFM Images of a 90nm AFM Calibration Grating .......................................................... 162

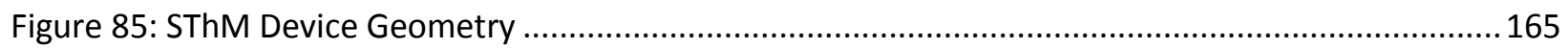

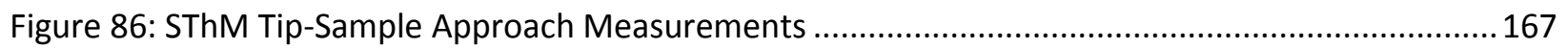

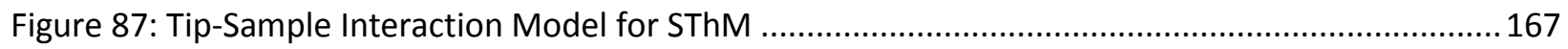

Figure 88: Time-Domain Temperature of SThM During Tip-Sample Approach..................................... 169

Figure 89: SThM Tip-Sample Approach. Left: Position of tip, Right: Temperature of tip....................... 169

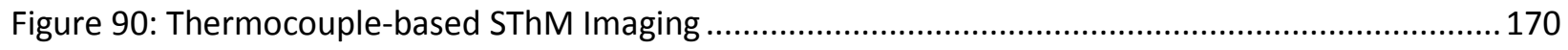

Figure 91: SThM Patterning With a Resistive Heater...................................................................... 171

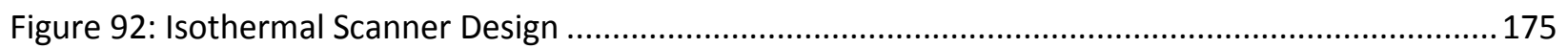

Figure 93: Amplified Strapped Chevron Actuator. FEA of Displacement and Blocked Force ..................179

Figure 94: Scanner Based on Amplified Strapped Chevron Actuators.................................................. 180

Figure 95: Depiction of a 2D Array of STM's (a), 1X5 Array of CMOS-MEMS STM's (b), Similar STM Array

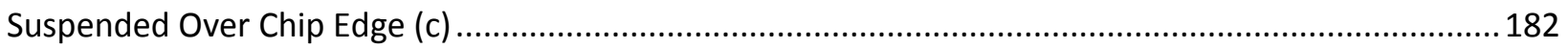




\section{List of Tables}

Table 1: The Development of Scanning Probe Microscopy Modes ........................................................ 10

Table 2: Material Properties Available in a CMOS-MEMS Process ..........................................................2 20

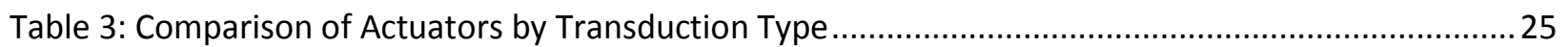

Table 4: FEA Simulation Results of Isothermal Scanner ................................................................... 176 


\section{Chapter 1. Introduction}

\subsection{Motivation}

The progress of technology from the Neolithic revolution to the Industrial revolution has always been concurrent with improvements in the precision and accuracy with which we are able to manufacture and inspect things. For roughly half a century, the microelectronics industry has enjoyed exponential scaling in critical dimensions, as predicted by the widely cited Moore's law [1]. Today the fabrication of features with dimensions of several hundreds of atoms has become routine, as semiconductor manufacturing has entered the nanometer regime. In contrast, conventional manufacturing, inspection, and metrology tools have not benefitted from dimensional scaling, and so they are encountering limitations in ultimate resolution, stability, and throughput. It is interesting to note that the cost-of-test for an individual transistor has exceeded the cost-of-manufacture per transistor, in part due to this disparity in scaling.

The concept of controlling the atomic structure of matter was proposed as early as 1959 by Richard Feynman in his renowned talk There's Plenty of Room at the Bottom:

"But I am not afraid to consider the final question as to whether, ultimately---in the great future---we can arrange the atoms the way we want; the very atoms, all the way down!"

Imaging individual atoms remained an elusive goal until the introduction of the scanning tunneling microscope (STM) in 1981 by Binnig, Rohrer, Gerber, and Weibel [2],[3]. This instrument provided for the first time the ability to view the atoms of flat samples in real space, and the history of its development was described by its inventors at their Nobel Prize lecture in 1987 [4]. In 1989, scientists marked a milestone in the ability to structure the world around us by demonstrating the ability to position individual atoms with atomic precision using an STM [5]. These landmark experiments ushered in numerous tip-based imaging modalities, known collectively today as the family of scanning probe microscopes (SPM's).

SPM's are widely regarded as the workhorse instruments of nanoscience. Their principle of operation is simple and elegant. A sharp tip is maintained in close proximity to a sample with a feedback controller that uses a tip-sample interaction measurement as its process variable. As the tip is scanned over the 
sample surface, the control effort is recorded. An image of the sample's surface structure thus emerges as a three dimensional plot of this control effort.

Conventional SPM's suffer from several drawbacks that present a barrier to their widespread adoption by industry. The most widely used SPM's are atomic force microscopes (AFM's). Commercially available AFM's rely on piezoelectric elements to scan a cantilever in 3-D. The use of these materials imposes several constraints on the system design. For instance, the strain produced by these actuators is small, so their size is several orders of magnitude larger than the achievable scan range. Large, bulky scanner designs are susceptible to thermal drift and poor vibration immunity. In addition, the mass of such scanners limits their mechanical bandwidth. Furthermore, the creep and hysteresis stemming from material properties in piezoelectric positioning systems manifest as image distortion. Although phenomenological and empirical models have been developed to mitigate creep, it remains a dominant parasitic effect at the nanometer scale. Closed-loop control of the scanners employing externally mounted capacitive position sensors can mitigate this issue; however, the tip-sample interaction is several millimeters away from the position sensor, introducing errors in positioning accuracy. The cantilever's position is typically read by a laser that is reflected from its rear surface. This imposes further constraints on the instrument design. For instance, if probes are to be operated in parallel to improve throughput, the system becomes prohibitively large due to the free-space paths of the optical configuration.

For SPM's to address the rigorous demands of metrology at the nanometer scale, they must be smaller, faster, and more stable.

\subsection{Thesis Statement}

This dissertation proposes a novel approach to the design and manufacturing of SPM instrumentation. More specifically, the thesis statement is:

All of the mechanical and electrical components that are required for a scanning probe microscope to capture an image can be scaled and integrated onto a single chip. The principles of electro-thermomechanical microsystem design can be applied to yield a chip-scaled instrument that independently acquires images of underlying samples, without the need for off-chip scanners or sensors. Furthermore, the performance such an instrument stands to benefit from integration and volumetric scaling of the scanner and sensor components by a factor of $1 \mathrm{E}-6$. 
The dissertation validates this thesis by reporting on the following contributions:

1) The development, fabrication and imaging results of the first single-chip contact-mode AFMs, with integrated piezoresistive strain sensing cantilevers and scanning in three degrees-offreedom (DOFs). The contact AFMs reported here may be divided into two categories based on their principles of operation:

a. Static AFMs that measure the topology of an underlying sample while maintaining a constant tip-sample interaction force

b. Quasi-static AFMs that use forced oscillation (off-resonance) of the cantilever and higher-harmonic detection of the signal from tip-sample interaction forces, to improve resolution in the presence of thermal disturbances

2) The design, fabrication and imaging results of the first single-chip dynamic AFMs, with integrated flexural resonant cantilevers and scanning in three DOFs. These dynamic AFMs may also be divided into two categories based on their principles of operation:

a. Amplitude Modulation AFMs (AM-AFMs) that maintain a constant amplitude of vibration in a resonant cantilever while in the presence of a force gradient from tipsample interaction

b. Frequency Modulation AFMs (FM-AFMs) that maintain a constant resonant frequency in an integrated cantilever while in the presence of tip-sample interaction force gradients.

3) A method to increase the quality factor ( $Q$-factor) of flexural resonators using an internal energy pumping mechanism that is based on the interplay between electrical, mechanical, and thermal effects. To the best of the author's knowledge, the devices that are designed to harness these effects possess the highest Qs reported for flexural resonators operating in air; $Q$ is enhanced from $\sim 50$ to $\sim 50,000$ in one exemplary device. A physical explanation for the underlying mechanism is proposed.

4) The design, fabrication, imaging, and tip-based lithographic patterning results with the first single-chip Scanning Thermal Microscopes (SThMs). These devices have 3 DOFs and integrated thermally isolated temperature sensors to detect heat transfer in the tip-sample region. Temperatures are measured with thermocouples and resistive temperature sensors.

5) The conception, fabrication and method of operation of an "isothermal electrothermal scanner." The mechanism, based on electrothermal actuators, maintains a constant temperature in a central location while positioning a payload over a range of $>20 \mu m$, thereby 
suppressing the parasitic thermal crosstalk effects that have thus far plagued thermally actuated devices.

6) The development of models to guide the design of single-chip SPMs and provide an interpretation of experimental results. The modelling efforts include:

a. Lumped element models for each component of single-chip SPMs in the electrical, thermal and mechanical domains.

b. Noise models for various components of the devices including temperature-based position sensors, piezoresistive cantilevers, and digitally controlled positioning devices.

\subsection{Outline of the Dissertation}

The remainder of this dissertation is outlined as follows.

Chapter 2 is a review of literature related to SPMs and MEMS. The origins of the types of SPMs that have been implemented with the CMOS-MEMS approach are discussed. Contributions from the MEMS community to scanning probe microscopy are then reviewed. The development of CMOS-MEMS processes is discussed.

Chapter 3 presents the models that are used throughout the design of chip-scaled SPM's. A lumped element model is derived for the following components of single-chip SPMs: self-heated resistors, lateral electrothermal actuators, flexural suspensions, thermal shunt paths and thermal isolation paths, vertical electrothermal actuators, flexural resonant cantilevers, piezoresistive strain sensors, and temperature sensors. The constitutive equations that govern the behaviour of these devices are discussed in Appendix $B$, and the details of the derivations for lumped element parameter values are discussed in Appendix C.

Chapter 4 presents experimental results of the $\mathrm{Q}$ enhancement that has been observed in the CMOSMEMS flexural resonant cantilevers used in single-chip SPMs. The models developed in Chapter 3 are used to interpret the $\sim 1000 \times$ Q-enhancement that has been observed under ambient conditions.

Chapter 5 discusses the driving and sensing strategies for closed-loop control of SPMs. Various methods to drive actuators are introduced. Methods to compensate for thermal crosstalk and to use temperature as a measure of position are introduced, and the relevant noise sources are quantified. The noise behaviour of piezoresistive sensors is also discussed. 
Chapter 6 focuses on contact AFM. Specifically, the design of devices for contact and higher-harmonic detection-based imaging is described. This chapter also aims to identify the deleterious effects that limit the resolution of single-chip SPMs. Once the effects are identified, the goal is to develop design strategies to maximize the signal-to-noise ratio (SNR) of the tip-sample force measurement. Characterization data for the instruments and images acquired in each mode are presented.

Chapter 7 focuses on dynamic AFM. Devices that are intended to operate as FM-AFMs and AM-AFMs are introduced. These devices contain incremental improvements that were implemented based on the observations from Chapter 6. Measurements of the device performance and images acquired in each mode are presented.

Chapter 8 introduces two types of SThMs. The first type makes use of thermocouples that are based on the Seebeck effect, while the second type is based on the TCR effect. The design specifics of SThM instruments are explained. Data from the operation of these instruments is presented and discussed, along with an image obtained with the thermocouple-based instrument. The TCR-based SThM is used to pattern a medium with a low melting temperature.

Chapter 9 reports on the operation of an "isothermal scanner" that may be used to improve the performance of single-chip SPMs. The design is based on modified chevron actuators with figures of merit that are an order of magnitude greater than previously reported CMOS-MEMS electrothermal actuators. When operated with the intended drive signals, an isothermal scanner maintains a constant average temperature over its entire positioning range. Operation of the isothermal scanner is reported, and its use for other types of SPM's is proposed.

Chapter 10 concludes with a comparison of the design principles and measured performance of CMOSMEMS scanners and SPM's. Potential applications of arrays of SPM's for patterning and metrology are discussed. Finally, methods to improve the resolution of each type of SPM are proposed for future work. 


\section{Chapter 2. Literature Review}

The contributions presented in this dissertation are multidisciplinary in nature; concepts are borrowed from a range of academic disciplines including surface science, electrical engineering, thermodynamics, and mechanical engineering. This literature review identifies specific works that form a foundation for the design of chip-scale SPM's, while pointing the reader to more comprehensive reviews of areas that are out its scope.

The chapter begins with a brief history of scanning probe microscopy, with emphasis on the modes that are implemented in this work. Then, some contributions from the field of MEMS to the surface science community are highlighted. The development of CMOS-MEMS processes like the one used in this work is then reviewed. Methods of actuation in MEMS are discussed and compared. The methods that have been developed to model electrothermal MEMS devices are then reviewed.

\subsection{Scanning Probe Microscopy}

The fundamental concept behind scanning probe microscopy is akin to the action of "feeling" an object; the idea is so intuitive that it is difficult to point to its origin. In an SPM, a sharp tip is moved in three dimensions over an object while measuring its interaction with the surface. The measured quantities are used to control the tip-sample separation, and they are stored and processed to reveal an image of the underlying sample. The physical implementation is reminiscent of the phonograph, invented in 1877 by Thomas Edison [6] and possibly the earliest example of a system that could track topology using a stylus to recover information. In 1928, Synge introduced the concept for a scanning probe near-field optical microscope [7]. In 1933, the concept of quantitative measurements of surfaces using stylus profilometry was introduced by Abbott and Firestone [8]. In 1972, the Topografiner [9] yielded the first measurements of surface microtopography using electronic tip-sample interactions. Imaging was performed using field emission currents, although tunneling currents were measured in a static mode of operation. The Topographiner was not sufficiently isolated from ambient vibration to produce STM images. Also in 1972, Ash and Nicholls used microwave radiation to demonstrate super-resolution imaging; a resolution of $150 \mu \mathrm{m}$ was obtained using radiation with $\lambda=3 \mathrm{~cm}$ (that is, $\lambda / 200$ ) [10].

The invention of the scanning tunneling microscope in 1982 [2] was quickly followed by the publication of atomic resolution images of silicon by its inventors [11]. Binnig and Rohrer received the Nobel Prize 
for their invention in 1986, and they are generally credited for the explosive growth of SPM's that followed. In the STM, a sharp, conductive tip is biased with respect to the sample to give rise to a

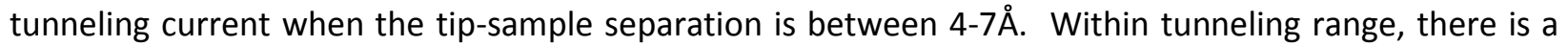
highly non-linear dependence of tunneling current on this gap, and this phenomenon is exploited to track topology. $A \sim 1 \AA$ change in the tip-sample separation results in a $\sim$ tenfold change in the tunneling current. Reviews of calculation methods for the tunneling current are presented in [12]. STM's require that the sample has a clean conductive surface, and they have been largely relegated to vacuum operation because many materials grow a native oxide under ambient conditions. Nevertheless, a large number of semiconductors and metals have been investigated using this invaluable tool [13].

In 1986, the AFM was invented by Binnig [14] and the first images acquired with the instrument were published by Binnig, Quate and Gerber [15]. In the first AFM, a diamond tip placed on a gold cantilever interacted with the sample. The tip-sample interaction force deflected the cantilever, and modulated a tunneling current that flowed between the cantilever and a sharp tip that was mounted on top of it. Although the inventors anticipated that the instrument could achieve atomic resolution, it took five years before Giessibl et. al. demonstrated it [16]. In addition to studies of insulating samples, the AFM can achieve atomic resolution under ambient conditions, unlike the STM which requires ultra-high vacuum except for a few special cases such as highly oriented pyrolytic graphite (HOPG). This is due to the fact that in air, the surface layer of solids constantly changes due to adsorption and desorption of atoms and molecules. Since there are no conductivity requirements in AFM, one can image virtually any flat surface without the need for surface preparation. Giessibl [17] provides a review of the common modes of operation in AFM, while Poggi [18] reviews several applications of the instrument.

The scanning thermal microscope (SThM) was invented in 1985 [19], and several means of tip-sample interaction measurement have been implemented [20]. The first SThM was based on the thermoelectric effect in a thermocouple sensor fabricated at the conical tip of a probe. Electrical resistance thermometry was introduced in [21], using a Wollaston wire shaped like a cantilever, enabling simultaneous thermal and AFM imaging. A surface micromachined thermal probe with integrated 1D electrostatic actuation was reported in 1997 [22]. A CMOS thermal probe with integrated piezoresistive detection is described in [23]. In contrast to the thermocouple probes, resistive probes can be used in passive and active modes. In the passive mode, a constant current is applied to a bridge circuit incorporating the sensor. The bridge voltage is recorded to measure the temperature. In the active mode, large currents flow through the resistor to induce joule heating. In the constant current mode, 
the tip-sample heat flux is detected as a change in probe resistance. In the constant temperature mode, a controller is used to maintain either constant resistance. This method has the advantage of improved bandwidth, since the probe does not have to reach thermal equilibrium. An interesting capability that was demonstrated in this mode is subsurface imaging [24]. A different technique for SThM involves thermal expansion measurements. This technique does not rely on temperature sensors that are located in the tip-sample region. In 1994, a bimorph cantilever was used as a sensor; temperature changes induced a bending moment in the cantilever that was measured using a conventional AFM. In the modes presented thus far, the tip-sample heat conduction reduces with tip sharpness. Scanning Joule Expansion Microscopy (SJEM) [25] is a mode that does not rely on tip-sample heat transfer, so there are no restrictions placed on the sharpness of the tip. In SJEM, a conductive sample is jouleheated with a periodic signal, and the material expansion is captured using lock-in techniques, with a conventional probe. Heated AFM [26] is yet another mode related to thermal tip-sample interactions; however, it does not directly reveal information about the heat transfer at the tip. Initially designated as an AFM cantilever for thermomechanical data storage [27], the authors have also reported topographic imaging with similar probes [28]. In heated AFM, the sharp tip is in contact with the sample, and is not used for heat transfer measurements. A large resistive heater with an appreciable TCR is located in the cantilever, and is cooled through the air gap to the sample. The local topology of the sample modifies this gap, which in turn affects the cooling of the heater. Thus, the resistance of the heater is used as a measure of topology. When the tip is sufficiently hot, samples may undergo a phase transition that modifies the air gap, indirectly revealing information about heat transfer at the tip [29]. This mode does not work in vacuum, and its vertical resolution is affected by the nature of sample topology under the heater.

The Scanning Microwave Microscope is the only type of SPM that can quantify local dielectric and conductivity measurements at radio frequencies. A microwave signal is fed to the tip, and the impedance of the tip-sample region changes the reflected signal to reveal images of the local electromagnetic properties of the sample. Ash and Nicholls [10] showed that the near-field resolution was determined by the size and geometry of the probe rather than wavelength, and that the Abbe barrier for resolution ( $\lambda / 2$ diffraction limit) could be broken in the near-field. One popular type of SMM employs a sharp tip protruding from the open end of a coaxial resonator with the other side coupled to a microwave source [30]. The frequency shift and change in quality factor were used to quantify electrical properties (dielectric constant and loss tangent or resistivity) of materials. Concurrently, this technique was augmented by shielding the open side of the coaxial resonator and opening a small area 
for the tip; this way, the electrical field could be confined near the tip and the parasitic capacitance is reduced to increase measurement sensitivity [31]. M. Tabib-Azar et al. used a stripline resonator and chemically etched a sharp tip to achieve $0.4 \mu \mathrm{m}$ resolution[32]. Lai et al. shielded the microwave signal using a stripline transmission line to improve spatial resolution by decreasing non-localized tip-tosample interaction [33]. Recently, Huber et al. introduced a calibrated SMM based on standard calibration samples for both capacitance and dopant profile measurements [34].

A large number of SPM's have been developed based on a range of tip-sample interaction mechanisms. Table 1 from [35] below contains a chronologically ordered listing of SPM modalities. Although a discussion of each type of SPM is beyond the scope of this dissertation, it should be noted that most of these modalities can be integrated in a CMOS-MEMS process. In fact, many of the modes are supported by the devices that are presented herein. For example, a CMOS-MEMS AFM with a shielded conductive path to its tip can be used as a scanning capacitance microscope, scanning attractive force microscope, "frictional" force microscope, electrostatic force microscope, scanning electrochemical microscope, or a Kelvin probe force microscope. Although the measurement setup for each type of microscope may vary, a similar device may be used in all modes. This example underscores the versatility of the CMOS-MEMS manufacturing process, which is the subject of section 2.3 . 
Table 1: The Development of Scanning Probe Microscopy Modes

\begin{tabular}{|c|c|c|c|}
\hline 1981 & Scanning tunneling microscope & G. Binnig, H. Rohrer & $\begin{array}{l}\text { Atomic resolution images of } \\
\text { conducting surfaces }\end{array}$ \\
\hline 1982 & $\begin{array}{l}\text { Scanning near-field optical } \\
\text { microscope }\end{array}$ & D. W. Pohl & $\begin{array}{l}50 \mathrm{~nm} \text { (lateral resolution) optical } \\
\text { images }\end{array}$ \\
\hline 1984 & $\begin{array}{l}\text { Scanning } \\
\text { microscope }\end{array}$ & J. R. Matey, J. Blanc & $\begin{array}{l}500 \mathrm{~nm} \text { (lateral resolution) images } \\
\text { of capacitance variation }\end{array}$ \\
\hline 1985 & Scanning thermal microscope & $\begin{array}{llll}\text { C. C. Williams, H. } & \text { K. } \\
\text { Wickramasinghe } & & \\
\end{array}$ & $\begin{array}{l}50 \mathrm{~nm} \text { (lateral resolution) thermal } \\
\text { images }\end{array}$ \\
\hline 1986 & Atomic force microscope & C. Binnig, C. F. Quate, Ch. Gerber & $\begin{array}{l}\text { Atomic resolution } \\
\text { conducting/non-conducting } \\
\text { surfaces }\end{array}$ \\
\hline 1987 & $\begin{array}{lll}\text { Scanning attractive force } \\
\text { microscope }\end{array}$ & $\begin{array}{l}\text { Y. Martin, C. C. Williams, H. K. } \\
\text { Wickramasinghe }\end{array}$ & $\begin{array}{l}5 \mathrm{~nm} \text { (lateral resolution) non- } \\
\text { contact images of surfaces }\end{array}$ \\
\hline 1987 & Magnetic force microscope & Y. Martin, H. K. Wickramasinghe & $\begin{array}{l}100 \mathrm{~nm} \text { (lateral resolution) images } \\
\text { of magnetic bits/heads }\end{array}$ \\
\hline 1987 & "Frictional" force microscope & $\begin{array}{l}\text { C. M. Mate, G. M. McClelland, S. } \\
\text { Chiang }\end{array}$ & $\begin{array}{l}\text { Atomic-scale images of lateral } \\
\text { ("frictional") forces }\end{array}$ \\
\hline 1987 & tic for & $\begin{array}{l}\text { Y. Martin, D. W. Abraham, H. K. } \\
\text { Wickramasinghe }\end{array}$ & $\begin{array}{l}\text { Detection of charge as small as } \\
\text { single electron }\end{array}$ \\
\hline 1987 & $\begin{array}{l}\text { Inelastic tunneling spectroscopy } \\
\text { STM }\end{array}$ & D. P. E. Smith, D. Kirk, C. F. Quate & $\begin{array}{l}\text { Phonon spectra of molecules in } \\
\text { STM }\end{array}$ \\
\hline 1987 & Laser driven STM & L. Ar & $\begin{array}{l}\text { Imaging by non-linear mixing of } \\
\text { optical waves in STM }\end{array}$ \\
\hline 1988 & $\begin{array}{l}\text { Ballistic electron emission } \\
\text { microscope }\end{array}$ & W. J & $\begin{array}{l}\text { Probing of Schottky barriers on a } \\
\text { nanometer scale }\end{array}$ \\
\hline 1988 & photoemission & $\begin{array}{l}\text { J. H. Coombs, J. K. Gimzewski, B. } \\
\text { Reihl, J. K. Sass, R. R. Schlittler }\end{array}$ & $\begin{array}{ll}\text { Luminescence } & \text { spectra } \\
\text { nanometer scale } & \\
\end{array}$ \\
\hline 1989 & Near-field acoustic microscope & $\begin{array}{l}\text { K. Takata, T. Hasegawa, S. Hosaka, } \\
\text { S. Hosoki, T. Komoda }\end{array}$ & $\begin{array}{l}\text { Low frequency acoustic } \\
\text { measurements on } 10 \mathrm{~nm} \text { scale }\end{array}$ \\
\hline 1989 & Scanning noise microscope & $\begin{array}{l}\text { R. Moller, A. Esslinger, B. } \\
\text { Koslowski }\end{array}$ & $\begin{array}{l}\text { Tunneling microscopy with zero } \\
\text { tip-sample bias }\end{array}$ \\
\hline 1989 & $\begin{array}{l}\text { Scanning } \\
\text { microscope }\end{array}$ & $\begin{array}{l}\text { Y. Manassen, R. Hamers, J. } \\
\text { Demuth, A. Castellano }\end{array}$ & $\begin{array}{l}1 \mathrm{~nm} \text { (lateral resolution) images of } \\
\text { paramagnetic spins }\end{array}$ \\
\hline 1989 & ion-conductance & $\begin{array}{l}\text { P. Hansma, B. Drake, O. Marti, S. } \\
\text { Gould, C. Prater }\end{array}$ & $\begin{array}{l}500 \mathrm{~nm} \text { (lateral resolution) images } \\
\text { in electrolyte }\end{array}$ \\
\hline 1989 & electrochemical & $\begin{array}{l}\text { O. E. Husser, D. H. Craston, A. J. } \\
\text { Bard }\end{array}$ & \\
\hline 1989 & $\begin{array}{l}\text { Absorption } \\
\text { microscope/spectroscope }\end{array}$ & J. Weaver, H. K. Wickramasinghe & $\begin{array}{l}1 \mathrm{~nm} \text { (lateral resolution) absorption } \\
\text { images/spectroscopy }\end{array}$ \\
\hline 1990 & $\begin{array}{l}\text { Scanning chemical potential } \\
\text { microscope }\end{array}$ & $\begin{array}{l}\text { C. C. Williams, H. } \\
\text { Wickramasinghe }\end{array}$ & $\begin{array}{l}\text { Atomic scale images of chemical } \\
\text { potential variation }\end{array}$ \\
\hline 1990 & Photovoltage STM & R. J. Hamers, K. Markert & $\begin{array}{l}\text { Photovoltage images } \\
\text { nanometer scale }\end{array}$ \\
\hline 1991 & Kelvin probe force microscope & $\begin{array}{l}\text { M. Nonnenmacher, M. P. O'Boyle, } \\
\text { H. K. Wickramasinghe }\end{array}$ & $\begin{array}{l}\text { Contact potential measurements } \\
\text { on } 10 \mathrm{~nm} \text { scale }\end{array}$ \\
\hline 1994 & $\begin{array}{l}\text { Apertureless near-field optical } \\
\text { microscope }\end{array}$ & $\begin{array}{l}\text { F. Zenhausern, M. P. O'Boyle, H. } \\
\text { K. Wickramasinghe }\end{array}$ & $\begin{array}{l}\text { Optical microscopy at } 1 \mathrm{~nm} \\
\text { resolution }\end{array}$ \\
\hline
\end{tabular}




\subsection{The Role of MEMS in Scanning Probe Microscopy}

The high volume, high yield manufacturing precision achieved by the semiconductor industry in the fabrication of integrated circuits (ICS) remains unsurpassed by any other technological endeavour. At the time of this writing, the production of $<20 \mathrm{~nm}$ thin lines is underway. The deposition, lithography and etching techniques borrowed from the IC industry have also enabled the batch fabrication of micromechanical components with exquisite dimensional control. Just as the improvement in IC performance is incremental [1], so too are the resolution of fabrication and metrology tools that address the challenges at this length scale. SPMs are the highest resolution tools available for imaging surfaces, and their performance is no exception; the instrument's speed, resolution, and stability all depend on improvements manufacturing precision of its constituents. For example, as cantilevers and scanners are miniaturized, the speed and stability of SPMs improves. In Ando [36], a small scanner with a first resonance of $60 \mathrm{kHz}$ and small MEMS cantilevers with resonance frequencies from $450 \mathrm{kHz}$ to $650 \mathrm{kHz}$ were used to generate video rate $(80 \mathrm{~ms} /$ frame) images of samples to capture the motion of molecules.

Progress in MEMS has had a profound impact on the SPM community, as a natural consequence of the laws of scaling that are discussed in Appendix A. Micromachining technology has played a significant role in the progress of AFM's, since the tip-sample interaction force is detected by the deflection of a cantilever beam. The first micromachined cantilevers were built at Stanford [37] out of $\mathrm{SiO}_{2}$ and $\mathrm{Si}_{3} \mathrm{~N}_{4}$. Today, single crystal silicon cantilevers based on a design presented in 1991 [38] are most common. In 1993, atomic resolution AFM images were obtained using a cantilever with an integrated piezoresistive strain sensor [39]. The optimization of a piezoresistive cantilever for AFM is discussed in [40]. MEMS cantilevers with integrated optical gratings are discussed in [41]. The FIRAT probe is a micromachined membrane that is actuated electrostatically, and includes an integrated diffraction grating. These gratings are illuminated by a laser, and the far-field patterns are measured with a photodetector. Cantilevers with integrated electrostatic actuation and capacitive detection are discussed in [42]. Heated AFM probes for thermomechanical data storage and topology imaging were presented in [43] and [44]. A piezoelectrically transduced cantilever was presented in [45]. A MEMS-based ring resonator with integrated transduction and $>1 \mathrm{MHz}$ natural frequency was discussed in [46]. Ultra-sharp tips can be integrated into silicon cantilevers with various methods, including oxide sharpening and anisotropic wet etching.

Probes that have been developed to support other modes of SPM also enjoy the benefits of microfabrication. A surface micromachined Polyimide thermocouple probe for SThM was presented in 
[47]. A silicon micromachined probe for KPFM was presented in [48]. Probes for MFM are discussed in [49] and [50]. Several probes for SMM have been described in the literature as well [51], [52], [53], [54], [55], [56], [33].

One approach to improving the bandwidth of SPM's is to use a fixed array of probes that are collectively scanned using a piezoelectric actuator. In this configuration, the probe usually incorporates a sensor for tip-sample interaction measurements, since the laser-based beam-bounce technique is not amenable to array operation. A $5 \times 5$ array of AFM's was implemented in a SOI (silicon on insulator) process incorporating piezoresistive sensing for each cantilever [57]. Data storage applications were investigated as part of the "Millipede" program at IBM [58]. A PZT cantilever array with piezoresistive detection was reported in [59]. The Millipede represents the largest and densest 2-D array (32X32, 1024 cantilevers) of AFM cantilevers to date. Electrostatically [60] and electrothermally actuated dip-pen nanolithography arrays have also been reported. Arrays of thermocouple probes were reported in [61]. A stand-alone single-chip AFM unit including an array of cantilevers with individual 1-DOF actuation, detection and control has been reported as well [62], [63]. It is important to note that all of these methods ultimately rely on a conventional AFM for scanning.

Several attempts have been made to incorporate the scanning and sensing functions on-chip. The first microfabricated STM device was a millimeter-scale piezoelectric multimorph with 3 DOF actuation, reported in [64]. This scanner achieved atomic resolution on an HOPG sample, with an impressive 20 frames/second imaging bandwidth. The maximum scan range of the device was $\sim 45 \mathrm{~nm} \times 300 \mathrm{~nm} x$ $7500 \mathrm{~nm}$, and its stiffness in the tip-sample approach direction was $3 \mathrm{~N} / \mathrm{m}$. Another approach is based on the SCREAM (Silicon Crystal Reactive Etching And Metallization) process developed at Cornell. The approach is based on high aspect ratio electrostatic comb drives for actuation and selective oxidation for the formation of tips and for lateral electrical isolation of suspended microstructures [65]. A scan range of $200 \mathrm{~nm} \times 200 \mathrm{~nm}$ was reported, along with an image of a step feature on a grating structure. Since these devices were intended for STM measurements, no sensors were required on the chips - ie, they are examples of scanners only. It is therefore not surprising that these were the first miniature SPM's. To the best of the author's knowledge, no other mode of SPM operation has been reported without the use of a conventional SPM for scanning. A 2-axis electrostatically driven kinematic stage with an integrated cantilever is discussed in [66]; the device does not incorporate a sensor, so imaging results were not achieved. A 2-DOF probe with an integrated JFET was presented in [67]. A CMOS-MEMS probe with open-loop 3-DOF positioning and integrated force sensing was presented in [68]. 


\subsection{CMOS-MEMS Processes}

There is a rich body of literature on various aspects of CMOS technology, including several texts on fabrication aspects [69],[70] . Process technology from a MEMS perspective is also the subject of several texts and review articles [71], [72], [73]. By appropriately combining the deposition, lithography and etch steps in CMOS with those needed for MEMS, it is possible to fabricate an integrated microsystem in a single process sequence. The devices we consider in this research are based on the co-fabrication of CMOS and MEMS, an area that is the subject of a number of commercial and academic research thrusts [74], [75]. A review of the development of CMOS-MEMS processes, with emphasis on strategies for robust co-integration, has been published by Fedder et. al. [76], and a book on the subject was published by Baltes et. al. [77] Mansour [78] provides an exhaustive review of CMOS-MEMS technology applied to RF applications.

When compared to conventional MEMS processes, the integrated approach offers several advantages. The excellent lithographical resolution in CMOS processes offers sub-micron minimum feature sizes that can be exploited by micromechanical structures. The local integration of pre-amplifiers with piezoresitive, temperature or force sensing tips stands to improve the signal-to-noise ratio of the measurement. Integrated electronics may be used to multiplex signals to and from the SPM's to reduce pin count, thus enabling the practical realization of large arrays of SPM's. The unique capability to integrate temperature and position sensors within actuators is used for closed-loop scanning operation.

The development of CMOS-MEMS approaches can be broadly categorized into three process integration strategies: MEMS-First processes, MEMS-Last processes, and MEMS in the CMOS Back End of Line (BEOL) stack (CMOS-MEMS processes). These are the subjects of next sections.

\subsubsection{MEMS-First processes}

The earliest applications that leveraged the integration of released microstructures and integrated electronics were pressure sensors fabricated using a MEMS-first approach[79]. In this strategy most of the MEMS-specific fabrication steps were performed prior to the electronics fabrication steps with the exception of the final device release steps. The release is typically the last process step since released microstructures would not withstand yield losses during standard semiconductor fabrication steps such as spin-coating of photoresist during the various patterning steps. Single crystal silicon (SCS) was used as the structural material, from which a diaphragm was etched using a wet anisotropic etchant. Transistors, resistors and interconnections were present on the device side of the wafer for increased 
signal transduction and reduced pin-count. Both capacitive and piezoresistive approaches were implemented.

In parallel with the pressure sensor development, a 10-mask process for micromachined silicon neural probes with integrated signal conditioning and multiplexing circuitry was also under development [80]. A heavily boron-doped region were used as an etch-stop to define the probe geometry, and dielectric passivation layers are used to protect the electronics from the wet etch. Recently, this process has been enhanced to fabricate wireless, RF-powered pressure sensors used to monitor arterial blood flow in what is considered to be one of the most sophisticated microsystems ever demonstrated [81].

MEMS-first processes consisting of more complex single crystal silicon microstructures combined with CMOS electronics have been enabled by the widespread availability of SOI wafers combined with the advent of DRIE processes for silicon [82]. In one early example, a DRIE step defined thick poly-Si structures over a buried oxide layer. SCS was epitaxially grown in the surrounding area, and bipolar transistors and interconnects were subsequently defined [83]. The fabrication of thick SOI microstructures with integrated electronics was greatly facilitated by the introduction of a trench-refill isolation process [84], [85]. In this approach, a first DRIE step defines trenches that are refilled with oxide. The wafer is then planarized prior to the CMOS fabrication steps. Upon completion of the electronics, a second DRIE step defines the microstructures that are finally released when the sacrificial (buried) oxide is removed. The SOl structures are left suspended from the walls of the isolation trench.

Thin film polysilicon structures can also be integrated with electronics using the MEMS-first approach [86]. The strategy adopted here begins with the definition of a trench in which the MEMS devices are deposited using surface micromachining techniques. The trench is then filled with a sacrificial oxide, and the wafer is planarized prior to CMOS fabrication. A final release step results in suspended microstructures that reside in the trench.

A desirable feature of MEMS-first approaches is for the MEMS devices to be released and encapsulated prior to the fabrication of electronics. This strategy prevents the CMOS from being exposed to the MEMS release steps, while protecting the released MEMS devices from subsequent CMOS processing. This feature has been implemented using LPCVD (conformally deposited) oxide or polysilicon to seal the holes in a shell that encapsulates the released MEMS device [87], and has yielded extremely stable resonators [88]. Microshell encapsulation was first demonstrated in the late 1980's using epitaxial 
silicon growth to seal a microresonator in vacuum, and several alternative approaches have been implemented [89].

\subsubsection{MEMS-Last Processes}

An alternate approach to the integration of CMOS and MEMS involves surface micromachining of the structural layers after completion of the CMOS back-end-of-line (BEOL) process steps. This strategy is truly modular and should be compatible with any IC fabrication process. Process options such as thick metallization for integrated RF passives have been available to designers for many years; MEMS-last processes are intended to have as negligible an impact on the CMOS front-end process as an additional interconnect layer. This way, circuit designs do not need to be modified. Storage [90] and display [91] applications have leveraged this process integration strategy. The damascene approach, commonly used in CMOS BEOL processes, has also been used to manufacture MEMS devices [92] and is being commercialized by companies like Microfabrica to enable prototyping of complex three-dimensional geometries using a variety of structural materials. This approach eliminates surface topology that would result from conformal deposition processes, and allows for many layers to be fabricated reliably as long as stress effects can be mitigated.

The primary constraint in MEMS-last processes is the thermal budget of the MEMS process. Although the CMOS transistors can withstand significant thermal processing, the metal interconnect layers suffer from increased contact/via resistance due to thermal stress and annealing effects. For a $0.25 \mu \mathrm{m}$ process, a thermal budget limit of $10 \mathrm{~h}$ at $425^{\circ} \mathrm{C}$ has been demonstrated [93]. For a $0.35 \mu \mathrm{m}$ process it was shown that the CMOS could tolerate up to 90 minutes at $525{ }^{\circ} \mathrm{C}$ [94]. Another constraint is the selection of a sacrificial material, since the CMOS passivation materials may be attacked in the release process. For instance, if $\mathrm{SiO} 2$ is used as a sacrificial material to be etched with $\mathrm{HF}$, the intermetal dielectric and passivation layers such as SiNx and polyimide would be attacked as well. Some alternative sacrificial materials are photoresist, polyimide, germanium and molybdenum.

The selection of structural materials varies by application. In the case of DLP technology, a metal (Al) structural layer with desirable optical properties was selected [91]. Gold has also been used for RFswitch applications and is being studied as a structural material for applications requiring low loss at high frequencies [78]. Piezoelectric materials have been integrated on top of the CMOS stack for RF applications such as surface acoustic wave (SAW) mechanical filters, thin-film bulk acoustic resonators (FBARs) or bulk acoustic wave (BAW) resonators [95], [96]. Zinc-oxide piezoelectric films have been 
shown to be compatible with CMOS processing, as demonstrated in an early multisensor chip [97]. Amorphous silicon (a:Si) devices have been integrated with CMOS in a MEMS-last approach using lowtemperature LPCVD [98] and PECVD [99]. In order to improve electrical conductivity, the LPCVD material was encapsulated in titanium-tungsten. PECVD a:Si technology has been developed to deposit transistors on plastic substrates at temperatures as low as 100 ㄷ. Polysilicon MEMS devices have also been deposited at high temperatures. The CMOS BEOL process had to be modified to withstand the high thermal budgets involved and unfortunately this approach resulted in unreliable source-drain contacts [100]. Polycrystalline germanium and polycrystalline silicon-germanium films were introduced as MEMS structural materials that could be deposited at low temperatures in the late 1990's as well [101]. Several other structural materials such as silicon nitride [102], vanadium oxide [103], polymeric structural materials [104], and carbon nanotubes [105] have been integrated with CMOS using the MEMS-last strategy.

\subsubsection{MEMS in the BEOL CMOS Stack (CMOS-MEMS)}

The existing materials in the CMOS metal-interconnect stack can be used as a structural material for MEMS devices, as was initially demonstrated in 1988 [106]. By stacking the via-cuts and omitting the underlying metal layers, an opening could be made in the dielectric stack all the way down to the substrate. A timed wet etch was then performed on the substrate in order to release devices. This process resulted in monolithic mechanical structures that could contain multiple electrically isolated metal layers in addition to gate and capacitor polysilicon. Early applications that utilized this integration strategy include thermal micro-hotplates with embedded polysilicon resistive heaters for chemical sensors [107] and infrared detectors with polysilicon/aluminum thermopiles [108].

Design rules in conventional CMOS processes do not allow the via-cut approach since they enforce fixedsize vias that must be overlapped by interconnect metal. A method introduced in 1996 [109] involved post-CMOS reactive ion etching of the dielectric stack and obviated via cuts through the use of the uppermost exposed metal layer as a mask. This method has been used on scaled CMOS down to the $0.18 \mu \mathrm{m}$ node with aluminum and copper interconnects, and is illustrated below in Figure 1 . The process is available through multi-wafer prototyping services with reasonable pricing and turnaround times. One variant of this process includes the addition of microfluidic channels in the BEOL layers by removing the metal with a liquid etchant [110]. Another enhancement incorporates the CMOS silicon substrate into microstructures. The most recent example of this approach made use of silicon DRIE to make bulk CMOS-MEMS. Front-side dielectric RIE defined etch pits to the substrate and backside DRIE was used to 
thin the wafer to a $\sim 40 \mu \mathrm{m}$ target thickness in selected areas [111]. This process allows the designer to mitigate stress-induced curvature of the BEOL layers by reinforcing plate structures with an SCS backing.

In this research, we have adopted a CMOS-MEMS process flow that includes a backside window through the use of diced grooves using a conventional dicing saw, or trenches using a laser dicing process. One advantage of this approach is its simplicity - a 2-step, maskless process is used to define structural layers and perform the release. Chips are shipped with the backside grooves already defined by dicing. Furthermore, the BEOL layers in a standard foundry CMOS process contain significantly more options than conventional bulk or surface micromachining processes. For instance the designer can define a 2 $\mu \mathrm{m}$ wide beam structure that contains a piezoresistive position sensor, a temperature sensor, a resistive heater, an actuator, and multiple electrically isolated signal lines. This level of integration is of great utility in the design of SPM instrumentation and is uniquely available in CMOS-MEMS processes.

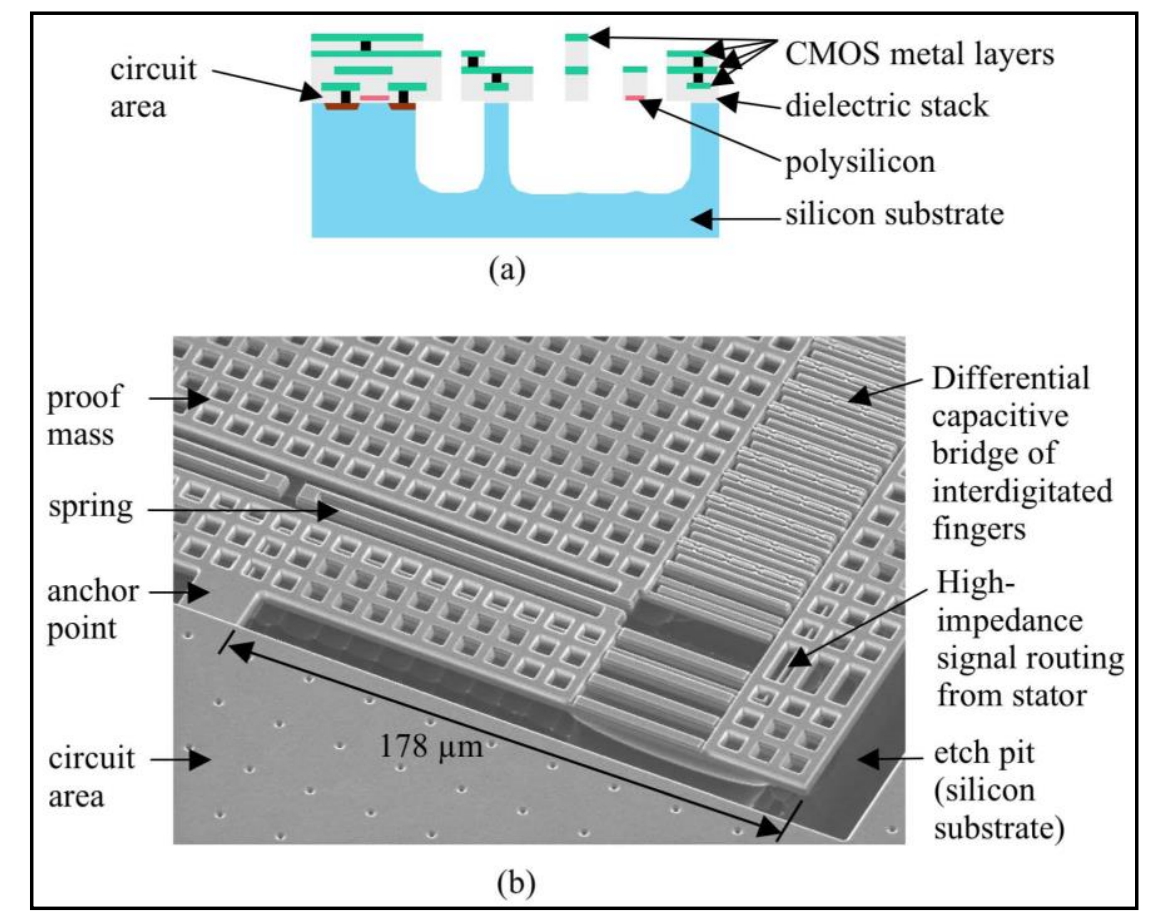

Figure 1: The ASIMPs (Application Specific Integrated MEMS Processes) CMOS-MEMS process results in released microstructures suspended over a cavity in the substrate. b) An accelerometer fabricated with the CMOS-MEMS process.

The disadvantages of the process include run-to-run and die-to-die variations in material properties such as stress. Stress reduction in newer processes ( 8 " wafers and beyond) combined with design patterns 
to compensate for stress-induced curvature are used to mitigate this issue. Another issue is the turnaround time for receiving released devices - although 3 months is reasonable for a process of this complexity, it is often extended to 4 months and impacts the design cycle significantly. Many foundries are beginning to offer CMOS-MEMS process options for the first time and several iterations will be required to identify issues and achieve consistently high yields for a given device.

\subsubsection{CMOS-MEMS for Single-Chip SPMs}

Chip-scaled SPM instruments rely on the concerted operation of various sub-components, each of which exploits a range of material properties. In this section, we illustrate that every aspect of the design of a CMOS-MEMS SPM leverages the material properties offered by the process, and conclude with some remarks about the values of the material properties in question.

Coefficient of thermal expansion: Electrothermal bimorph actuation is used to position the tip in three dimensions. Two materials with different coefficients of thermal expansion (CTE's or $\alpha$ ) are required. The larger the difference, the better the performance of the actuator. A figure of merit for the material selection in single material electrothermal actuators has been expressed in [112]: $F O M=\sigma / k E$, where $\sigma$ is the thermal expansion coefficient, $\mathrm{k}$ is the thermal conductivity, and $\mathrm{E}$ is the Young's Modulus.

Sheet Resistance: The resistive material that is used for joule-heating in actuators should have appropriate sheet resistance. The value of the resistor determines the amount of voltage that is required to drive the actuator. CMOS compatible voltages are desirable, since the drive circuitry can be implemented on-chip. At a fixed voltage, a lower value resistor will generate more heat because Joule heating process is a function of power, and $P=V^{2} / R$. In addition, the resistance of a sensor impacts its noise performance, as will be shown in Chapter 5.

There are conflicting constraints on the temperature coefficient of resistivity (TCR). For joule-heating, this value should be as low as possible to prevent "thermal runaway" conditions and nonlinearities in the transfer function of the actuators, which will be discussed in Chapter 5. However, for the purpose of resistive temperature sensing, this value should be maximized.

The Seebeck coefficient gives rise to a temperature-related voltage at the junctions between different conductors. This quantity should be maximized for temperature sensitivity in SThM, but minimized to mitigate thermal noise in sensors. A figure of merit for thermopower in materials is given in [113], $\mathrm{x}=\mathrm{S}^{2} / \mathrm{k} \rho$. 
The piezoresistivity of a material should be maximized to sense strain in a beam for application in AFM. In other types of sensors, a low piezoresistivity may be desirable to suppress strain-induced drifts.

Both dielectric and conductive materials are needed in order to route signals to sensors and actuators in a chip-scaled instrument. The dielectric materials should have low leakage currents, high breakdown voltages, and good mechanical properties if they are to be used as structural materials. The conductors should have low resistance and good mechanical properties.

The Young's Modulus is an important mechanical material property that influences the stiffness of a beam and hence its natural frequency. Although a high Young's modulus is desirable in some aspects of the design of chip-scaled SPM's, it lowers the actuation range. The relative Young's modulus of materials in a bimorph is used to set the geometric ratio of the layers. If the Young's moduli are the same, both materials can be designed to have the minimum feature size in the case of a lateral actuator.

The thermal conductivity $\kappa$ affects thermal time constants related to heating or cooling a volume of material. Insulating materials are needed to reduce power consumption at the expense of bandwidth. The opposite is true for conductive materials. The specific heat capacity $c_{\rho}$ of a material has the opposite effect; it is proportional to the amount of heat required to raise the temperature of the material. The thermal diffusivity combines these material properties $D=\kappa / \rho c_{\rho}$, and may be thought of as a thermal analogy to an RC time constant.

A native oxide layer grows on many materials under ambient conditions. Noble metals are an exception, and are desirable for applications that require conductive junctions like ohmic contacts for probing, or STM tips. CMOS process do not generally offer noble metals as an option.

Electrical noise in semiconductors is a multivariable effect. The resistivity, size, and cleanliness of an electrical resistor all contribute to the noise spectrum of an electrical resistor.

The residual stress in deposited materials should be kept low in order to mitigate out-of-plane deflection and buckling of flexures and actuators that are intended to operate in-plane. However, stress gradients are useful in the self-assembly of out-of-plane actuators.

Table 2 is a list of these material properties, along with their values in several MEMS processes. 
Table 2: Material Properties Available in a CMOS-MEMS Process

\begin{tabular}{|c|c|c|}
\hline Design Feature & Material Property & $\begin{array}{c}\text { Range of Values in CMOS-MEMS } \\
\text { Processes }\end{array}$ \\
\hline Lateral and vertical bimorphs & CTE, $\alpha^{1}$ & Al: 23.1, Oxide: $0.5, \mathrm{~W}: 4.5{ }^{*} \mu \mathrm{K}^{-1}$ \\
\hline Vertical bimorphs & Residual Stress, $\sigma_{0}{ }^{2}$ & $\begin{array}{l}\text { Oxide: }-485 \text { to }+250, \mathrm{Al}:+73 \text { to } \\
+232 \text {, Poly: }-583 \text { to }+203 \mathrm{Mpa}\end{array}$ \\
\hline Lateral Flexures, Resonant Cantilevers & Youngs Modulus E ${ }^{3}$ & $\begin{array}{l}\text { Si: 155-180, Poly: } 130-175, \text { Al: 69, } \\
\text { Oxide: } 57-70 * \text { Gpa }\end{array}$ \\
\hline Lateral Flexures, Lateral Actuators & Aspect Ratio $^{4}$ & Up to $10: 1$ (released) \\
\hline Resistive heater & Resistivity $^{5}$ & 0.76 to $90 * 10^{-3} \Omega \mathrm{cm}$ \\
\hline Resistive Thermometer & $\mathrm{TCR}^{6}$ & -5.0 to $+4.38 * 10^{-3} / \mathrm{K}$ \\
\hline Thermocouple (Seebeck) & $\alpha_{\mathrm{s}}$ & $\begin{array}{c}\text { Poly: }-366.9 \text { to }+380, \mathrm{Al}:-1.7 \\
\qquad \mu \mathrm{V} \mathrm{K}^{-1}\end{array}$ \\
\hline Integrated Piezoresistor & Gauge Factor, GF & Poly: 5-17 PPM $\varepsilon^{-1}$ \\
\hline Dielectric structural layer (isolation) & Dielectric Breakdown Voltage $^{7}$ & Intermetal, >200V, Gate: >9V \\
\hline Thermal shunt path, isolation path & Thermal Conductivity $\mathrm{K}^{89}$, & $\begin{array}{l}\text { Oxide: } 0.5 \text { to } 1.5 \text {, Poly: } 18 \text { to } 45.6 \text {, } \\
\text { Al: } 237, W: 174,{ }^{*} \mathrm{~W} \mathrm{~m}^{-1} \mathrm{~K}^{-1}\end{array}$ \\
\hline Sharp, Conductive tips & Native Oxide & All materials have native oxide \\
\hline Electrothermal actuators & Volumetric Heat Capacity, $\rho c$ & $\begin{array}{l}\text { Al: 2.4. Oxide: } 1.25 \text { to } 1.5 \\
\quad * 10^{6} \mathrm{~K} \mathrm{~m}^{-3} \mathrm{~K}^{-1}\end{array}$ \\
\hline Bulk Piezoresistor & Piezoresistive coefficient & $\begin{array}{l}\pi_{44}: p-S i: 62 \text { to } 138, n-S i:-19 \text { to }-9.7 \\
\pi_{11}: n-S i,-38 \text { to } 102.2 p-S i, 1.1 \text { to } 6.6\end{array}$ \\
\hline
\end{tabular}

\footnotetext{
${ }^{1}$ A large difference in CTE causes a greater bending moment upon heating

${ }^{2}$ Residual stress is needed to generate a self-assembled out-of-plane displacement. This is required for the tip to engage the sample in one type of approach.

${ }^{3}$ A high Young's Modulus improves the out-of-plane stiffness of lateral flexures and increases the resonant frequency of cantilevers

${ }^{4}$ A high aspect ratio increases the linear range of flexures and prevents out-of-plane buckling under moderate loads

${ }^{5}$ The resistivity determines whether the desired heater resistance can be obtained in a reasonable footprint

${ }^{6}$ The TCR of a resistive heater should be low to avoid thermal runaway and non-linearity in the transfer function of the actuator

${ }^{7}$ A structural insulating material is critical as it allows for electrical routing throughout the instrument to various sensors and actuators

${ }^{8}$ A material with high thermal conductivity can be used to provide thermal shunt paths from "hot-spots" to the substrate, and reduce thermal coupling to nearby sensors

${ }^{9}$ A material with low thermal conductivity can be used to thermally isolate structures from one another, or from the substrate (to improve efficiency at the cost of bandwidth)
} 
In addition to material properties, there are constraints on the device geometry.

The device thickness has profound impacts on performance. A thin layer is useful to concentrate stress in piezoresistive regions. Thick layers are used to improve out-of-plane stiffness and to suppress curling effects.

The layer-to-layer registration is critical in the design of lateral bimorph actuators. Several layers must be aligned in order to generate a common thermal moment to deflect a beam.

The minimum feature size of a released structure has profound consequences, as it bounds the scaling factor and the aspect ratio that can be achieved. The aspect ratio of structures in a MEMS process is of fundamental importance in the design of flexures. A high aspect ratio enables in-plane flexures with appreciable linear ranges, and also suppresses buckling effects.

To summarise, the design of single-chip SPMs exploits a range of material properties and features that are available in CMOS-MEMS processes. However, there is an important caveat: none of these properties are optimized with SPMs in mind. The goal of a CMOS process is to improve the quality and yield of CMOS electronics, which are intended to perform consistently over a wide range of temperature and be insensitive to strain from packaging. These requirements dictate a low TCR and gauge factor. The residual stress in CMOS processes must be lowered to mitigate bowing issues as wafer sizes scale upwards. These are examples of CMOS requirements that are in contradiction with the needs of singlechip SPMs. Furthermore, the values that are tabulated above are not all available in a single process. They represent the overall range that is available in a variety of MEMS processes, as measured and presented in [77]. Thus, although piezoresistivity, a Seebeck coefficient, some residual stress and a TCR are all available for the MEMS designer to exploit, none of these values are comparable to those obtained in a process that has been optimized for sensing these effects. This reality contributes challenges to the design of CMOS-MEMS SPMs.

\subsection{Micro-Scale Actuators}

Actuators enable MEMS to perform physical functions and interact with their environments by altering geometry at the micron scale; they are the point at which energy is converted into force and motion. In most cases, an input is converted from the electrical domain to a non-electrical output signal in the radiant, magnetic, thermal, mechanical, or chemical domains. 
In the design process of a microsystem that incorporates actuation several parameters are specified, including: output force, displacement, rise-time, power consumption and overall size. Some metrics that have been used to evaluate existing techniques include the maximum strain that can be produced by the transduction mechanism, and the maximum work-to-volume ratio that can be attained. The designer must consider all actuators that satisfy the design constraints and make the optimal selection.

Reviews of existing actuator techniques have categorized and discussed their driving principles [114], [115]. Here we briefly discuss transduction principles, and evaluate and compare the performance of the actuators.

Common electrothermal methods of actuation include bimorph actuators (single and multi-material), bent-beam or chevron actuators [116], [117], topology optimized structures, and various types out-ofplane actuators [118], [119]. These devices are further discussed in later sections as they were selected for the SPM implementations that are the focus of this research.

The volumetric expansion associated with phase changes in materials has been exploited in a large number of MEMS actuators. Microactuation using thermally driven phase changes to induce volume expansion has been shown to generate high forces in small volumes; both liquid-air and solid-liquid material systems have been explored [120], [121]. Both devices make use of resistive heaters to induce the phase transformation, and a flexible membrane to enclose the material while allowing displacements.

Another means of actuation that makes use of phase transformations is the shape memory effect, which is exhibited by shape memory alloys (SMA) such as TiNi [122], [123]. This thermally induced crystalline transformation between a ductile (Martensitic) phase and a high strength (Austenitic) phase has been harnessed in MEMS with the use of thin film deposition techniques such as dual target sputtering. It is important to note that at the micro scale, the limiting factor in the response time of these devices is not the thermal mass, but rather the time constant associated with the phase transformation. Nevertheless, some benefits of scaling are still evident, such as reduced power consumption. SMA devices exhibit some of the highest work-to-volume ratios among micro-actuators. In the design of SMA actuators, a restoring force must be integrated in order to deform the device while it is in the martensitic phase to prepare for the next cycle of operation. 
Optical actuation using photo-induced phase transition materials such as polydiacetylene can exhibit significant induced strains [124]. The resulting bimorph-like actuation can generate forces and displacements, but with limited efficiency.

Thermopneumatic actuation based on volumetric expansion in a single phase has been used to produce valves that can sustain differential pressures of $\sim 1 \mathrm{MPa}$ [125], with Fluoroinert ${ }^{\mathrm{TM}}$ liquid as the working material.

Photothermal actuation achieves localized heating with incident light that is focused onto a radiationabsorbing microstructure [126]. A large surface area is typically required to convert optical energy from a focused beam into thermal energy, and this reduces the work/volume ratio. The conversion process is reported to have an efficiency of $\sim 50 \%$ due to Fresnel reflection losses and mismatches between the laser wavelength and finite absorption peaks of the material.

Actuators based on electrostatic principles include scratch drive actuators (SDA's) [127], [128] comb drives [129], [130], parallel plate designs [131],[132] membrane deformation actuators [133], [134], Sshape flexures [135], distributed force actuators [136], [137], and torsional/gimbaled actuators [138]. The SDA can produce high forces over large distances due to the untethered nature of the device; however, the lifetime of the device is severely limited to $\sim 600 \mathrm{~mm}$ of travel due to the quality of thin films that are used to electrically isolate the substrate from the device layers, as well as catastrophic failure due to stiction caused by tribocharging. The work-to-volume ratio of these devices is the same as parallel plate devices, since the field geometry is similar. Distributed force actuators such as the integrated force array can produce larger displacements at the expense of a higher volume. Although the work-to-volume ratio of comb-drives is moderate, their resonant amplitude, efficiency, and frequency of operation make them suitable for many applications that do not require mechanical work, such as accelerometers, optical devices and RF devices. Finally, the torsional actuator is used in micromirror arrays incorporated in commercial products such as projection displays. The lifetime of the mirrors exceeds billions of cycles, and switching speeds are on the order of milliseconds.

Impact drive mechanisms [139], [140], [141] and electromagnetic actuators [142], [143] have been implemented at micron scales as well. Magnetically anisotropic materials such as nickel-iron films and polymer magnets can be electroplated or otherwise deposited onto microstructures and subsequently actuated by external fields [144], [145]. Magnetostrictive materials experience strains when an external 
magnetic field is applied. The strains that are produced by this phenomenon have been used to deflect a bimorph [146], [147], [148].

Table 3 has been adapted from Krulevitch [123], Carlen [121], and Fujita [114] in order to quantitatively compare the various actuation methods and provide some additional details. When actuators are designed to do mechanical work, a useful figure of merit is the work per unit volume, W. The static displacement and force exerted by an actuator are used to calculate work, and divided by the volume occupied by the device. Resonant motion amplitude is not considered since the actuator is intended to bear a load. It should be noted that for many RF and optical applications of MEMS, the frequency of operation is critical and a resonant mode of operation is desired, since the interaction with electromagnetic fields does not load the actuator.

The properties of CMOS-MEMS materials support various electrostatic, electrothermal, and electromagnetic actuation options. Among these options, only electrothermal and electromagnetic actuators can provide the desired displacements at CMOS-compatible drive voltages. Electromagnetic actuators made of CMOS-MEMS materials require an external magnetic field. Thus, among all types of MEMS actuation, the requirements for integration are uniquely possessed by electrothermal actuation.

This research employs lateral and vertical thermal bimorph actuators comprising aluminum and silicon dioxide as the driving materials. An important advantage of this approach is its low voltage requirement, which is suitable for CMOS integration; on-chip circuits can provide the voltages $(<5 \mathrm{~V})$ and currents $(>1 \mathrm{~mA})$ that are needed. Another consideration is that the available materials for electrothermal actuation have favorable properties. Al and $\mathrm{SiO}_{2}$ have similar Young's Moduli, a large difference in thermal expansion coefficients, and a large difference in thermal conductivity. The implications of these properties will be emphasized in Chapter 3.

The lateral electrothermal actuators used in this work can provide a large (20 $\mu \mathrm{m})$ static displacement; this quantity impacts the achievable scan-range of the SPM. With the use of certain design patterns, the out-of-plane actuators can achieve $>40 \mu \mathrm{m}$ range. The out-of-plane range of motion sets the maximum vertical elevation of sample features that can be imaged. Since the vertical range scales with the square of length $\left(L^{2}\right)$, while the stiffness scales with $1 / L^{3}$, there is a tradeoff between range and stiffness. It is common to combine several vertical actuators in parallel to increase stiffness while maintaining the same range of motion. The position resolution of the actuator is also inversely related to its range of motion. This has profound consequences on the operation of the instrument. A high speed, high resolution instrument requires a stiff actuator with limited vertical scan range. At the 
Table 3: Comparison of Actuators by Transduction Type

\begin{tabular}{|c|c|c|c|c|}
\hline Actuator type & Implementation Details & $\begin{array}{l}\text { Work-per-volume } \\
\text { equation }\end{array}$ & $\mathrm{W} / \mathrm{V}\left(\mathrm{J} / \mathrm{m}^{3}\right)$ & $\begin{array}{l}\text { Frequency of } \\
\text { operation }(\mathrm{Hz})\end{array}$ \\
\hline \multirow[t]{6}{*}{ Phase Change } & Solid-liquid & $\frac{1}{-}\left(\frac{\Delta V}{v}\right)^{2} \kappa$ & $1.0 \times 10^{7}$ & 1 \\
\hline & Liquid-vapour & $\frac{1}{3}\left(\frac{\Delta V}{V}\right)^{2} \kappa$ & $2.03 \times 10^{4}$ & \\
\hline & Austenite-Martensite & $\sigma \cdot \epsilon$ & $2.5 \times 10^{7}$ & 75 \\
\hline & Austenite-Martensite & $\sigma \cdot \epsilon$ & $6.0 \times 10^{6}$ & \\
\hline & Microbubble & $\frac{F \cdot \delta}{V}$ & $3.4 \times 10^{2}$ & 100 \\
\hline & Optically induced & $\sigma \cdot \epsilon$ & $4.74 \times 10^{1}$ & \\
\hline Thermopneumatic & External pump source & $\frac{F \cdot \delta}{V}$ & $1.2 \times 10^{6}$ & 5 \\
\hline \multirow[t]{4}{*}{ Thermal } & $\begin{array}{ll}\text { Single } & \text { material } \\
\text { expansion } & \\
\end{array}$ & $\frac{1}{2} E(\alpha \cdot \Delta T)^{2}$ & $4.6 \times 10^{5}$ & \\
\hline & Bimorph & $1 K_{\text {bimorph }} \cdot \delta^{2}$ & $4.0 \times 10^{4}$ & \\
\hline & & $V$ & & \\
\hline & Optically heated & $\frac{1}{2} E(\alpha \cdot \Delta T)^{2}$ & $4.74 \times 10^{1}$ & \\
\hline \multirow[t]{3}{*}{ Electromagnetic } & Ideal & $\frac{-M_{S}^{2} \cdot A \cdot \delta}{2 \mu \cdot V}$ & $4.0 \times 10^{5}$ & $1 \times 10^{5}$ \\
\hline & Permalloy & $\frac{-M_{S}^{2} \cdot A \cdot \delta}{2 \mu \cdot V}$ & $2.8 \times 10^{4}$ & $2 \times 10^{2}$ \\
\hline & Solenoid & $\frac{N}{V}$ & $1.6 \times 10^{3}$ & $1 \times 10^{3}$ \\
\hline Magnetostrictive & Cylinder & $\frac{3}{2} \frac{\lambda_{S} \sigma}{V}$ & $1.0 \times 10^{7}$ & $1 \times 10^{3}$ \\
\hline \multirow[t]{3}{*}{ Electrostatic } & Parallel plate, SDA & $\frac{\varepsilon v^{2} A \delta}{2 g^{2} V}$ & $1.8 \times 10^{5}$ & $1 \times 10^{5}$ \\
\hline & Comb drive & $\frac{\varepsilon v^{2} l \delta}{g V}$ & $3.4 \times 10^{3}$ & $1 \times 10^{4}$ \\
\hline & Integrated force array & $\frac{\varepsilon v^{2} A \delta}{2 g^{2} V}$ & $7.0 \times 10^{2}$ & $2.5 \times 10^{4}$ \\
\hline \multirow[t]{2}{*}{ Piezoelectric } & PZT & $\frac{1}{2}\left(d_{33} E_{f}\right)^{2} E$ & $1.2 \times 10^{5}$ & $1 \times 10^{6}$ \\
\hline & Zno & $\frac{1}{2}\left(d_{33} E_{f}\right)^{2} E$ & $1.8 \times 10^{2}$ & $1 \times 10^{7}$ \\
\hline Muscle & & $\frac{F \cdot \delta}{V}$ & $1.8 \times 10^{4}$ & 5 \\
\hline
\end{tabular}

$\boldsymbol{V}$ : material volume

$\Delta \boldsymbol{V}: \quad$ change in material volume

$\boldsymbol{\kappa}$ : bulk modulus

$\boldsymbol{\sigma}$ : mechanical stress

$\boldsymbol{\epsilon}: \quad$ is the mechanical strain

$\boldsymbol{F}$ : $\quad$ external driving force

$\boldsymbol{\delta}$ : displacement under actuation

$\boldsymbol{E}: \quad$ Young's Modulus

$\alpha: \quad$ coefficient of thermal expansion

$\Delta \boldsymbol{T}: \quad$ temperature change

$\boldsymbol{K}_{\text {bimorph }}$ : bimorph stiffness
$\boldsymbol{M}_{\boldsymbol{S}}$ : $\quad$ permanent magnetic bias

$\boldsymbol{A}: \quad$ electrostatic electrode area

$\boldsymbol{\mu}$ : $\quad$ permeability

$N$ : number of turns

$\lambda_{s}: \quad$ magnetostriction

$\varepsilon$ : permittivity

$\boldsymbol{v}$ : voltage potential

$\boldsymbol{g}$ : $\quad$ gap between electrostatic electrodes

$\boldsymbol{l}$ : $\quad$ electrostatic electrode width

$\boldsymbol{d}_{33}$ : piezoelectric coefficient

$E_{f}$ : $\quad$ electric field 
system level, the vertical scan range of the device places a constraint on the position resolution of the coarse approach mechanism. In addition to the aforementioned qualities, electrothermal actuators also enjoy small footprints and ease of fabrication in a CMOS-MEMS process.

This work relies heavily on the performance of electrothermal actuators. The final geometry of a given design is influenced by a range of constraints, including: range of motion, mechanical bandwidth, stiffness, operating temperature, position resolution, power efficiency, footprint, sensitivity to thermal crosstalk, and sensitivity to external vibrations. A model of the electrothermal actuator should therefore be capable of predicting these quantities, and their sensitivity to design parameters. Appendix $B$ provides a review of the methods that have been used by various authors to capture the behaviour of electrothermal actuators. Chapter 3 provides an overview of the lumped element models that have been developed over the course of this work. 


\section{Chapter 3. Development of Coupled Electrothermomechanical Models}

This section develops models that predict quantities in the electrical, thermal and mechanical domains for various components of chip-scaled scanning probe microscopes. Each of the many parts of a chipscaled SPM has performance specifications that are influenced by effects in these three domains. To build a model for a single element, one must consider solutions of the constitutive equations in each domain, and the nature of the coupling between domains (intra-domain coupling). The components are further interconnected to one-another in various ways; the SPMs consist of many elements that are coupled within each of the domains (inter-domain coupling). In this chapter, lumped element models of various components are developed using analytical expressions in Appendix B as a starting point. When a lumped element model fails to capture important distributed effects, the models are enhanced. The models are then interconnected to shed light on how the coupling between elements impacts device performance. A comprehensive model of the SPM therefore seeks to capture the interplay of the components within various domains; the model ultimately serves to guide the design process to enhance the desired effects and suppress the parasitic ones.

Every component of the CMOS-MEMS SPMs can be modeled using lumped element equivalent circuits according to the principles described by Senturia [149]. In the electrical domain, the use of the $e \rightarrow V$ convention implies that the voltage is the across variable, and the current is the through variable. In the mechanical domain, the $e \rightarrow V$ convention implies that the force is the across variable, and the velocity is the through variable. In a circuit representation, a capacitor replaces the compliance of the spring that stores potential energy, while an inductor is the analog of the mass that stores kinetic energy. The effort and flow variables are conjugate power variables, meaning that their product in a given circuit element is equal to the power in that element. In the thermal domain, the thermal convention implies that the temperature is the across variable, and the heat current is the through variable. A thermal capacitor stores heat energy, and a thermal resistor represents the reciprocal of thermal conduction. The effort and flow variables in the thermal convention are not conjugate power variables, so one must use the heat energy to keep track of energy flow.

This chapter begins with a discussion of the components of a CMOS-MEMS SPM. For each component, the dominant effects contributing to device performance are first identified from analytical expressions, and then directly incorporated into a lumped-element representation. Experimental results are the most quantitative way to evaluate these devices, followed perhaps by Finite Element Analysis (FEA), both of which were heavily used over the course of this work. However, it is often the most simplistic 
model with a minimal set of parameters that can provide the required insight to guide the design process. The focus of this chapter is to develop such models and to elucidate the effects that contribute to and detract from the performance of single-chip SPMs.

\subsection{Components of a CMOS-MEMS SPM}

At a glance, while a CMOS-MEMS SPM is scanning a sample, the nature of each distinct building block in the instrument is obscured by its monolithic construction undergoing continuous, coordinated flexural deformations. The behaviour of such a system, simultaneously responding to a multitude of inputs and generating a multitude of outputs, would be difficult to capture in a single continuum model. In fact, the meshing process alone for such a device could take several hours. The problem becomes tractable when it is divided into various sub-components that are each intended to serve a single purpose.

The elements of a scanning probe microscope that are modeled in this work are: self-heated resistors, lateral quasi-static electrothermal actuators, flexural suspensions, thermal shunt paths and thermal isolation paths, vertical dynamic electrothermal actuators, resistive and thermocouple-based temperature sensors, flexural resonant cantilevers, and piezoresistive strain sensors. The last two components in this list are used in conjunction to produce the effects that are the focus of Chapter 4 , so their modeling is deferred to the next chapter.

A description of the model development for each component follows.

\subsection{Self-Heated Resistor}

Joule heating that occurs in self-heated resistors is the source of power for all of the actuators used in this work. The heaters are treated as localised sources of heat, and their placement within the actuator influences the time and position dependent temperature distribution. Because the polysilicon material used in CMOS processes possesses a TCR, the local temperature influences the electrical resistance. This is a source of feedback from the thermal domain to the electrical domain, and it results in a nonlinearity in the voltage-to-position transfer function of the device. A more significant non-linearity stems from the fact that the heat power is proportional to the square of the voltage input. Both of these effects are captured in the lumped element model.

\subsubsection{Electrical Model}

The electrical domain representation of the resistor is simply a voltage source and two resistors. The first resistor value is the room-temperature value of the polysilicon resistor that is embedded within an 
actuator. The second resistor value captures the temperature dependence of the polysilicon material. A circuit diagram of the electrical domain representation is shown in Figure 2.

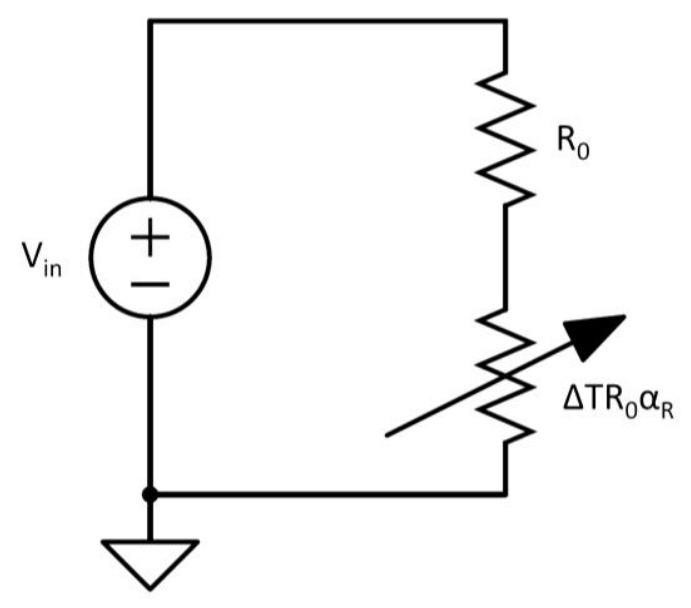

Figure 2: Electrical Domain Representation of a Self-Heated Resistor

\subsubsection{Thermal Model}

The full analytical solution of the heat equation in the thermal domain reveals the temperature distribution in the resistor. The heat conduction problem is solved with a spatially constant source term that represents Joule heating, fixed temperature boundary conditions that represent conductive cooling to the substrate, and a temperature-dependent sink term that represents convection to the atmosphere.

For the development of compact models, the time-dependent temperature distribution in a self-heated resistor may be calculated using several methods. One approach is to discretize the one-dimensional heat equation using finite difference methods. In the DC steady-state, a network of resistors may be used to model heat conduction. This approach reveals the steady-state temperature distribution, but does not capture the thermal time constant of actuators under quasi-static operation. With the addition of heat source elements (voltage sources) and heat capacity elements (capacitors), a large number of elements may predict the temperature distribution and frequency response. Yet another approach is to calculate the impulse response of the heater, expressed in terms of eigenfunctions that match the boundary conditions. A lumped RC circuit and impulsive source can represent each mode. A small number of modes can accurately predict behaviour up to moderate frequencies. However, when the heater is loaded with a thermal mass or more complex boundary conditions are imposed, the eigenfunctions need to be recalculated. Thus, this method is most useful for simple geometries. Finally, 
the device may be represented by a single resistor to account for conductive cooling, a single resistor to account for convection, and a single capacitor for the heat capacity of the device. Although this approach may accurately predict the average temperature of the heater, it carries no spatial information about the temperature distribution.

In the lateral and vertical actuators used throughout this work, the heater resistors may be considered as localised sources of heat current, so the simple lumped element representation is appropriate because the spatial information is not important. A model that includes these thermal elements is shown in Figure 3. Although this method provides reasonable estimates of the heat flow, the actual temperature is dependent on the placement of the resistor within the actuator. The model must reflect this, as illustrated in the following example.

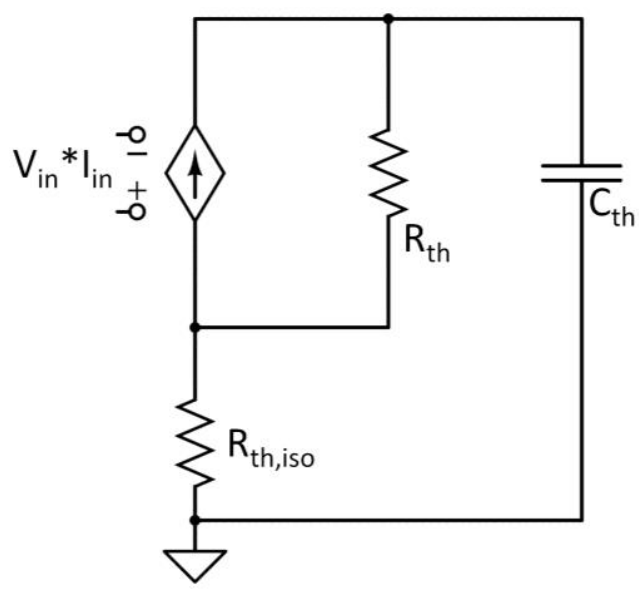

Figure 3: Thermal Domain Representation of a Self-Heated Resistor

Consider the vertical bimorph actuators with embedded heater resistors shown in Figure 4. The two devices are identical, except for the resistor placement. In the first example, the heater is located near the base of the actuator, such that the thermal resistance to the substrate is low. In the second example, the heater is located near the tip, such that the thermal resistance is high. For the purpose of illustrating temperature distribution under DC steady-state conditions, the bimorph itself may be modeled simply as a thermal resistor. The tip heater and base heater are placed $100 \mu \mathrm{m}$ and $1 \mu \mathrm{m}$ away from the substrate anchor, so the resistance values are $100 \Omega$ and $1 \Omega$ respectively.

The results of static FEA are shown in Figure 4 along with values for the temperature and deflection of each device. The maximum temperature, average temperature and overall deflection of the tip-heated 

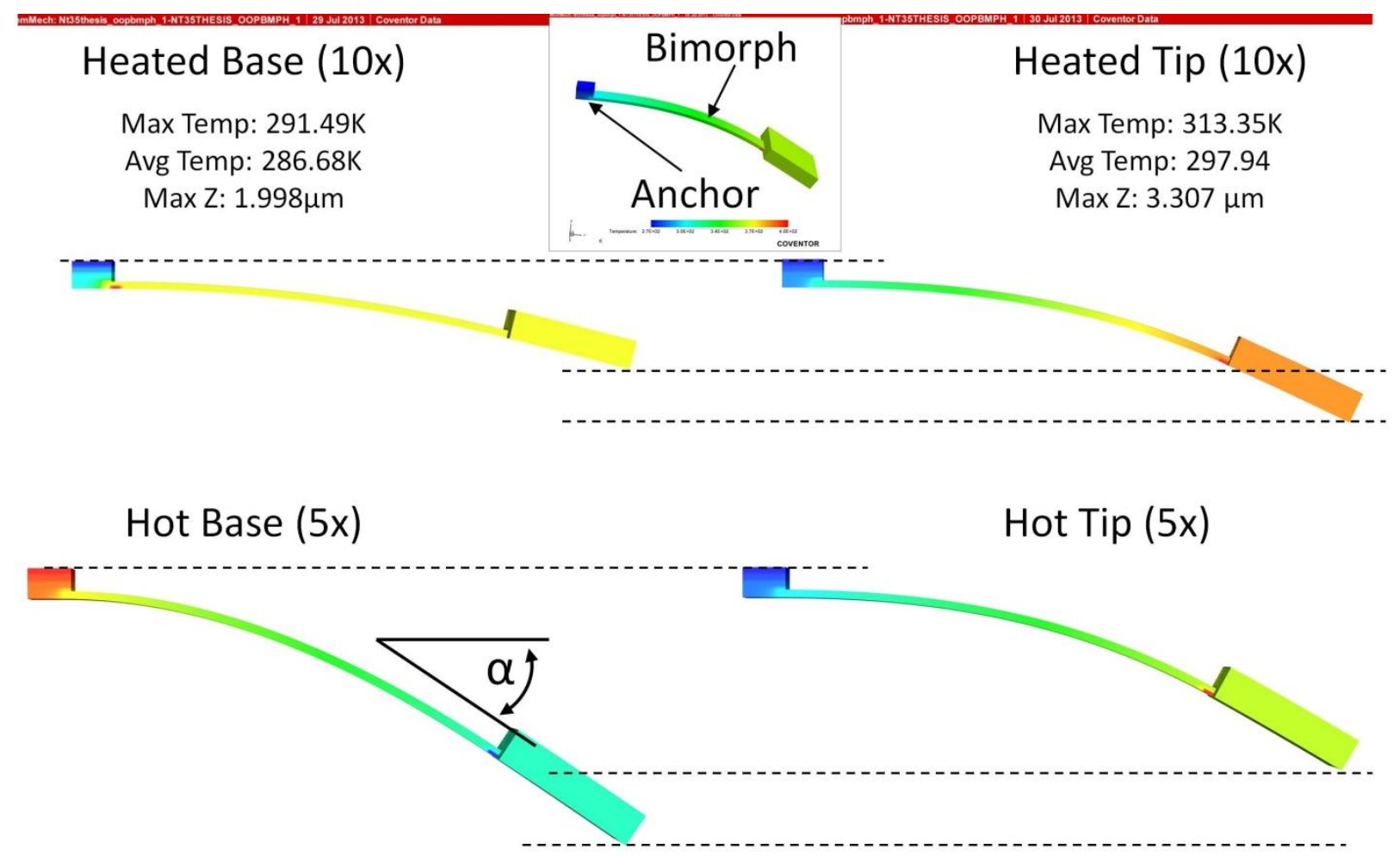

Figure 4: FEA Simulation of Bimorph Under Base (left) and Tip (right) heating

device are higher than those of the base-heated device. Since the electrical power input is identical, the tip-heated device is more efficient under DC steady state conditions.

It is interesting to note that if the heat sources are replaced with fixed temperature boundary conditions, the behaviour of the actuator is reversed; that is, the bimorph with the hot tip is less efficient than the bimorph with the cold tip. Although both bimorphs have almost the same average temperature of $\sim 325 \mathrm{~K}$, the device with the hot tip deflects $8 \mu \mathrm{m}$, while the other device deflects $11.3 \mu \mathrm{m}$. In the figure, the two situations are denoted as "heated" vs. "hot" conditions, where heated refers to the presence of a heat source and hot refers to a temperature boundary condition. Note also that the tip angles in the "hot" case are very close, implying that if one were only interested in the tip angle (as in micromirror applications), the average temperature would be a sufficient parameter to simulate.

The cold tip / hot base condition may seem like an unlikely scenario, since the anchor to the substrate is usually the dominant cooling path. However in this work, there are two scenarios in which this temperature distribution occurs. Under dynamic operation, the AC component of the temperature decays exponentially away from the heater due to the thermal capacitance of the beam. This effect is pronounced when the frequency of operation is higher than the corner frequency of an actuator, as in 
the case of resonant cantilevers. Another scenario is when vertical actuators are placed at the distal end of lateral actuators. If the vertical actuator is off while the lateral actuator is on, convective cooling dominates for the vertical actuator, so the tip may be colder than the base. This DC case will be considered in the development of vertical bimorph actuator models, where it will be shown that the temperature gradient over the length of the actuator must be considered. The AC case will be considered in the development of resonant cantilevers.

\subsubsection{Voltage-source Drive}

The electrothermal model of a self-heating resistor is shown below in Figure 5. A voltage source provides the input to the device.

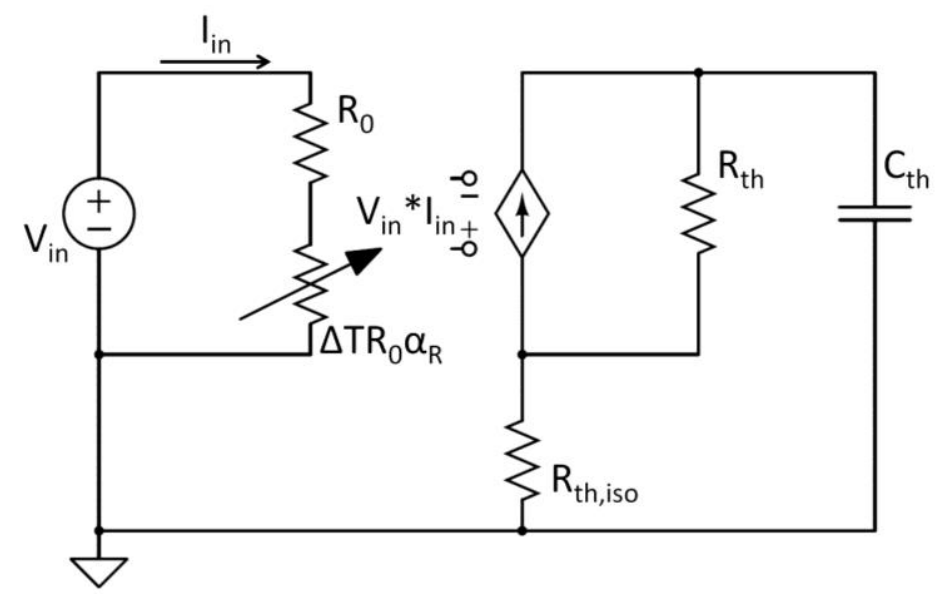

Figure 5: Electro-Thermal Representation of a Self-Heated Resistor

The governing equation of this first order system is [149]:

$$
C_{t h} \frac{d T_{R}}{d t}=-\frac{T_{R}}{R_{t h}}+\frac{V^{2}}{R_{0}\left(1+\alpha_{R} T_{R}\right)}
$$

This expression contains two non-linearities that affect the voltage-to-temperature (and therefore voltage-to-position) transfer function of actuators that are used in the SPM's. The first non-linearity is the $V^{2}$ dependence, which may be removed with a non-linear inversion by taking the square root of the voltage signal before it is applied to the heater. The second non-linearity results from the presence of the temperature term in the denominator below $V^{2}$. Since the TCR is small, the following approximation can be made: 


$$
\frac{V^{2}}{R_{0}\left(1+\alpha_{R} T_{R}\right)} \approx \frac{V^{2}}{R_{0}}\left(1-\alpha_{R} T_{R}\right)
$$

Substituting back into (3.1) and collecting terms gives:

$$
\frac{d T_{R}}{d t}=-\frac{1}{R_{t h} C_{t h}}\left(1+\frac{\alpha_{R} R_{t h} V^{2}}{R_{0}}\right) T_{R}+\frac{V^{2}}{R_{0}}
$$

The thermal time constant for this system, assuming a constant $V^{2}$, is:

$$
\tau_{V}=\frac{R_{t h} C_{t h}}{\left(1+\frac{\alpha_{R} R_{t h} V^{2}}{R_{0}}\right)}
$$

which depends on the value of the input voltage. The steady-state temperature rise of this linear system with negative feedback is:

$$
T_{s S}=\frac{\frac{R_{t h} V^{2}}{R_{0}}}{\left(1+\frac{\alpha_{R} R_{t h} V^{2}}{R_{0}}\right)}
$$

If the resistor has a positive temperature coefficient, the value of the denominator term increases with applied voltage, implying that the slope of $T_{S S}(V)$ decreases, and stable operation is possible. For negative temperature coefficients, the situation is reversed, and there is a possibility of "thermal runaway" at sufficiently high voltages. The term "thermal runaway" is used to describe the situation in which there is positive feedback between the input voltage and the temperature, making it possible for the temperature to rise at a fixed input voltage. Beyond a characteristic threshold voltage, this would result in catastrophic device failure due to overheating.

Figure 6 below shows measured results of the resistance variation of self-heated resistors as a function of applied voltage. A resistive ladder circuit consisting of a fixed resistor and the device under test is used to measure the current and voltage simultaneously to compute the unknown resistor value. In one device, the resistor is encased in a rigid plate that does not experience significant out-of-plane deflection. The temperature coefficient of voltage (TCV) of this device is negative. In another device, the resistor is placed in a thin beam that curls upon the application of heat (only the Metal 1 layer is used, resulting in a bimorph actuator). The TCV in this case is positive, even though the polysilicon material is the same. The reason for this discrepancy is the fact that the second device experiences 
compressive stress as a result of the bending in the beam, an effect that will be discussed further in Section 4.2. Thus, the TCR of the material cannot be inferred from self-heating if the resistor experiences heat and strain simultaneously.

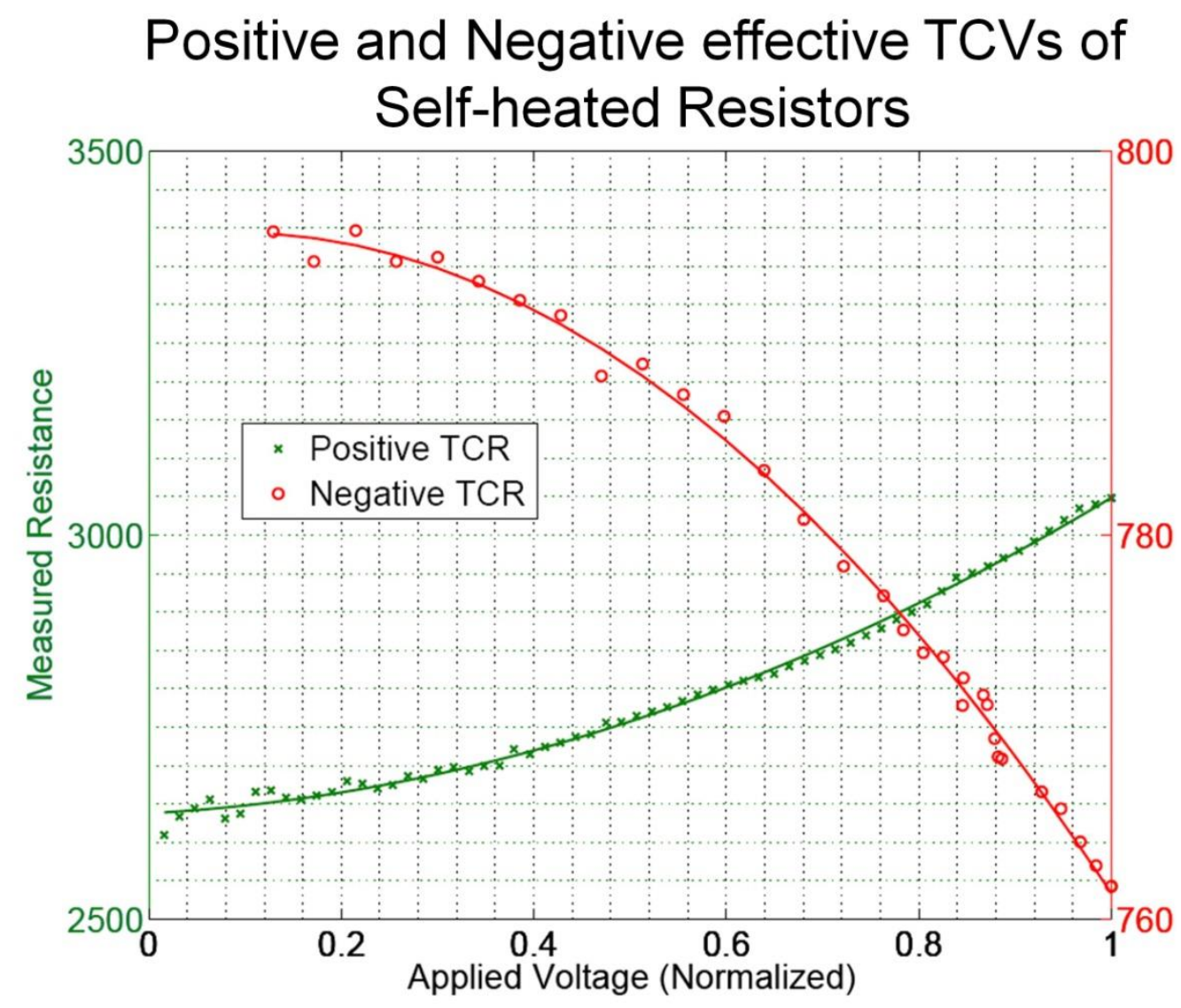

Figure 6: The temperature coefficients of resistivity in CMOS polysilicon are strain-dependent

Based on the data, one can extract meaningful quantities related to the TCR and piezoresistivity of the material as follows. To measure the TCR, the stress-free resistor is placed in a microclimate oven. The temperature dependent data is matched to the second order polynomial fit from Figure 6 to obtain a power coefficient of resistivity (PCR) for stress-free material. This coefficient relates the linear dependence of temperature on the applied power. To extract piezoresistance, one must first calculate the strain in the beam that is to be measured. In most cases, a Metal-1 beam is used for piezoresistive sensing, so the placement of the polysilicon layer with respect to the neutral axis of the beam is known. The second order polynomial fit of the bimorph data from Figure 6 can then be used to extract a PCR for strained materials. In the case of this material in the Metal-1 bimorph geometry, the effect of piezoresistivity is $2.3 x$ higher than that of the TCR, with the opposite sign. Thus, for a bimorph beam in 
static deflection, the strain dependence dominates over the temperature dependence and reverses the TCP. It is important to note that the magnitude of compressive strain in the resistor increases with temperature (and applied power), because the bimorph bends downwards and the polysilicon layer is placed below the neutral axis of the beam. Therefore, the coefficient of piezoresistivity of the material is negative, since it is defined with respect to tensile stress.

\subsection{Lateral Electrothermal Actuators}

Lateral electrothermal actuators are used to scan the tip over the sample's surface. Two types of actuators have been investigated in this work: multimorph actuators that are based on the bimorph effect, and chevron actuators that are based on single-material expansion. The methods developed in this section may be applied to both types of actuators, by choosing the appropriate conversion from the thermal to the mechanical domain. This discussion focuses on the multimorph actuator implementation shown in Figure 7. The composite beam consists of materials that are present in the BEOL steps of the CMOS fabrication process. In the layout of the beam, the lower metal layers are intentionally offset from the top metal layers in order to obtain the cross-section shown in the figure. Since the TEC's of Aluminum and $\mathrm{SiO}_{2}$ are different, a temperature increase results in a thermally generated mechanical moment along the length of the beam, causing the multimorph to deflect in the plane of the substrate (laterally). The moment is the effort source, and mechanical potential energy is stored in the deflected beam.

A single bimorph beam is not sufficient to provide the force and out-of-plane stiffness that are required for SPM operation. It is therefore desirable to couple several beams together. Because the tips of the bimorphs deflect in an arcuate fashion, it is not possible to simply connect them with a plate at the distal end, since this would impose a zero-moment constraint and eliminate any deflection from bending. The design pattern in Figure 7 is used to cancel the rotation at the tips by combining the beams in a symmetrically reflected fashion, such that they may be attached. The overall deflection achieved with such a design is $1 / 2$ of what can be achieved with a full-length bimorph. Because of the vertical asymmetry in the cross section of the beams, a vertical deflection arises due to a thermal moment in the y-direction. This parasitic out-of-plane motion is undesirable because it is in the tipsample direction, and could result in significant image distortion. The out of plane bending is canceled by way of another reflection, as shown. The end result is a lateral actuator with stiffness and output force that scales with the number of cascaded beams in the design. The lumped element model may be 
adjusted with multiplicative factors to incorporate the increased number of beams and the effects of reflections on the design.

Before delving into the model development, it should be noted that several unique design constraints are applied to lateral actuators for SPM scanners.

1. Resonant Frequency: The objective of the scanner is to raster-scan the tip of the SPM in the plane of the sample surface. The regime of operation of the actuators is referred to as "quasistatic" because they produce large static displacements at high scanning speeds (relative to conventional scanners), but they do not operate in mechanical resonance; nevertheless, a high mechanical resonant frequency is desirable to suppress the excitation of vibrations from external sources. In dynamic AFM, a source of vibration is the resonance of the vertical actuator, which will be discussed in section 3.5. External sources of vibration include building vibrations $(<1 \mathrm{kHz})$, acoustic vibrations $(<10 \mathrm{kHz})$, and vibrations from laboratory equipment $(<1 \mathrm{kHz})$. The resonant frequency of the lateral actuators used in this work is above $50 \mathrm{kHz}$.

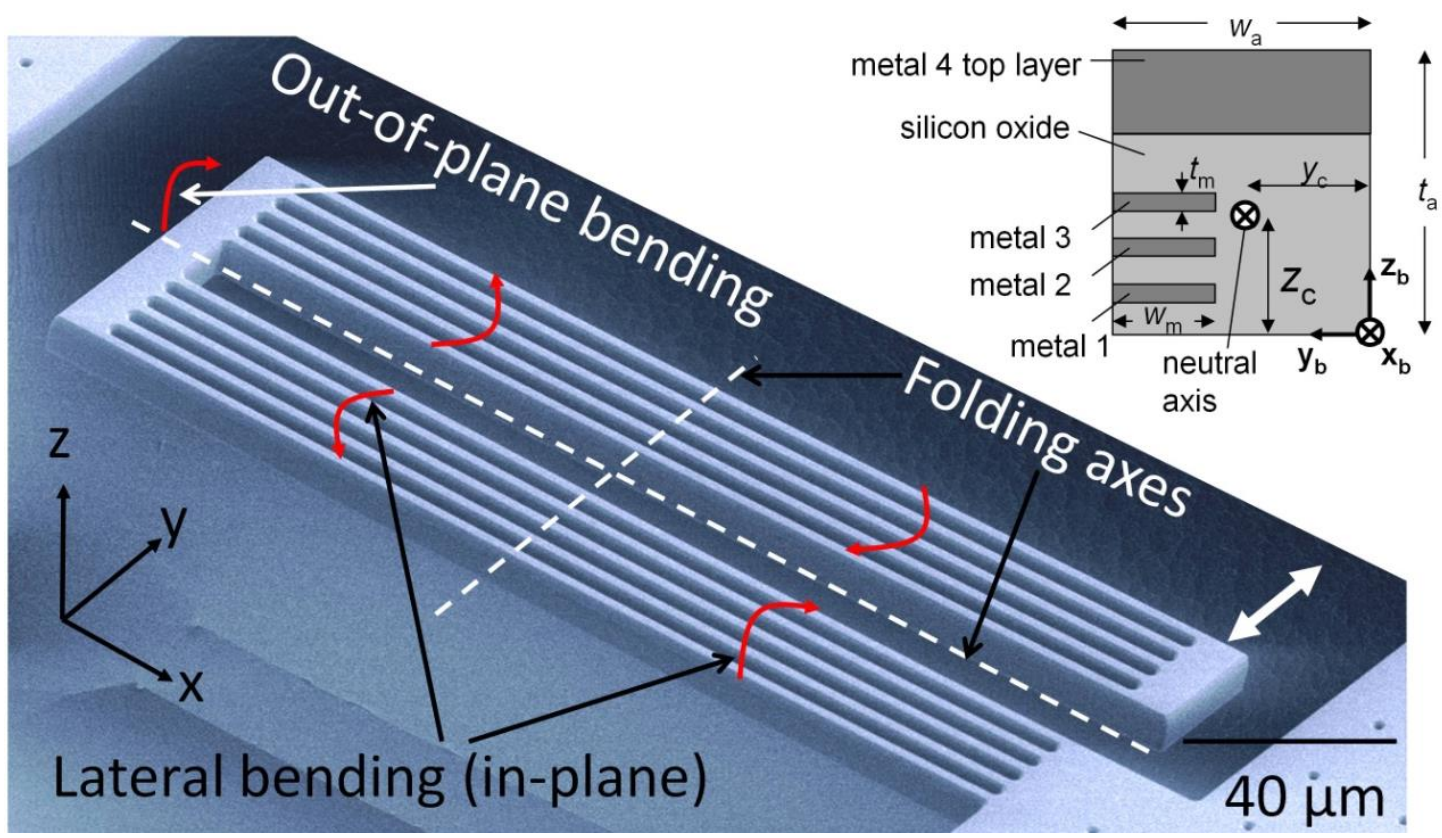

Figure 7: Lateral Multimorph Geometry. The cross-section of the beam contains lower metal layers that are laterally offset from the top metal layers in order to create a bending moment. Beams are folded to match in-plane tip rotation and out of plane curl. 
2. Thermal time constant: Another constraint is that the velocity of the scan is should be controllable, since the user may wish to increase the scan speed without introducing aberrations or reducing the scan window. Thus, the actuators should be capable of a range of operation frequencies without compromising their range or linearity. The maximum frequency of operation of thermal actuators is limited by their thermal time constants, which are related to how quickly heat can be added or removed from their thermal mass. The thermal time constants of the actuators used in this work range from $0.1 \mathrm{~ms}-10 \mathrm{~ms}$, depending on their thermal mass and thermal resistance. This translates to maximum frequencies of operation of $100 \mathrm{~Hz}$ to $10 \mathrm{kHz}$, and thus enables video-rate scan speeds: a $10 \mathrm{kHz}$ actuator can produce an image with 800 lines at 25 frames per second. The rate limiting factor for imaging then becomes the bandwidth of the tip-sample interaction measurement, instead of the scanner dynamics that may be a bottleneck in conventional instruments.

3. Range of motion: A third constraint is introduced if the user wishes to zoom to a particular location in the image, such that the actuators must be capable of maintaining a static offset deflection. The multimorphs used here can produce up to $20 \mu \mathrm{m}$ of static deflection. If larger static deflections are required, flexures may provide a geometric advantage to increase the scan range; however, this reduces the overall stiffness and resonant frequency of the SPM.

4. The out-of-plane stiffness places a fourth constraint on the lateral actuators, as they are in the mechanical path between the tip and substrate. Depending on the type of tip-sample interaction, the overall stiffness requirements of the system may range from $0.1 \mathrm{~N} / \mathrm{m}$ (contact AFM) to $10-50 \mathrm{~N} / \mathrm{m}$ (STM, FM-AFM).

5. A fifth constraint is that the operating voltage should be compatible with the CMOS process, so that driver circuitry can be integrated on-chip. The actuators require $<5$ volts, and the value of the heater resistor can be reduced easily to lower the operation voltage further at the expense of increasing current consumption.

6. Another constraint is that the maximum operating temperature of the actuators cannot exceed thermal budgets of $\mathrm{CMOS}$ processes, since the BEOL materials will delaminate at high temperatures (above $\sim 350^{\circ} \mathrm{C}$ ).

7. The power efficiency influences many aspects of the performance of electrothermal actuators. A high efficiency actuator will reach greater temperatures at a given input power. Efficiency may be increased by thermally isolating the actuator from the substrate. It will be shown in 
section 5.3 that improving the efficiency of the actuator lowers the minimum detectable displacement in the presence of noise.

8. Sensitivity to thermal crosstalk is related to power efficiency. If an actuator is isolated from the substrate but not from neighboring devices, it will be more sensitive to thermal crosstalk. To improve signal to noise ratio, actuators should have a short thermal path to ground and be isolated from one another.

\subsubsection{Electrical Model}

The electrical model of the actuator calculates the amount of joule heating in a resistor that is subject to an applied voltage. The temperature-dependence of this resistor is captured through feedback from the thermal domain. The $\mathrm{V}^{2}$ dependence of heat generation is captured in the thermal domain. This is identical to the model of a self-heated resistor embedded in a rigid plate, as discussed in the preceding section.

\subsubsection{Thermal Model}

The thermal model for a lateral actuator must capture the temperature distribution along the length of the beam. Several observations can be made from the solution to the heat problem[150]. First of all, the solution takes the form of a dissipating thermal wave with a propagation constant and penetration depth. As the frequency increases, the penetration depth decreases. We denote the turnover frequency $f_{t}$ as the frequency at which the thermal wave does not reach the end of the beam. At this frequency, the beam is considered "thermally long." The constriction of the temperature distribution is the reason that thermal actuators have a limited bandwidth, beyond which the amplitude rolls off like a single-pole RC transfer function. A second observation is that the phase of the temperature oscillations varies along the length of the beam. As the frequency is increased, the phase along the length of the beam may vary by 180 degrees, meaning that the moment applied at the base of the beam is opposite in phase when compared to the smaller moment applied at the tip. This detracts further from the attainable deflection at higher frequencies. A third observation is that the amplitude of the temperature variation near the tip of the beam is much smaller than at its base. This intuitive result implies that sensors prone to thermal disturbances should be located far away from the heaters, especially when heaters are operated at a frequency for which the beam is thermally long.

The development of a lumped element model that captures thermal effects begins with a finite difference approximation of the 1-D heat equation as detailed in Appendix B.2. The beam is assumed to 
have a constant cross-section with isotropic thermal conductivity $\kappa$, density $\rho$, and heat capacity $c_{p}$. For the beam with the geometry shown in Figure 7, the values for these parameters are calculated as:

$$
\begin{gathered}
\kappa=\frac{1}{w_{a} t_{a}} \sum_{\text {members }} \kappa_{i} \Delta \mathrm{y}_{i} \Delta \mathrm{z}_{i} \\
\rho=\frac{1}{w_{a} t_{a}} \sum_{\text {members }} \rho_{i} \Delta y_{i} \Delta z_{i}, \quad c_{p}=\frac{1}{w_{a} t_{a}} \sum_{\text {members }} c_{p_{i}} \Delta y_{i} \Delta z_{i}
\end{gathered}
$$

A capacitor captures the time dependence of the temperature, implemented as the current through a capacitor with a value of:

$$
C_{t h}=2 A \rho c_{p} \Delta x
$$

The thermal conductivity between nodes is represented as a resistance with the value:

$$
R_{12}=R_{23}=R_{\text {Cond }}=(\Delta x) / \kappa A
$$

A third resistor represents heat loss through convection:

$$
R_{\text {conv }}=\frac{T_{a}-T_{2}}{i_{c o n v}}=\frac{1}{2 w \rho c_{p} h_{c o n v} \Delta x}
$$

One section of the thermal model shown in Figure 8 does not include any heat sources, since the selfheated resistors are placed in discrete locations outside of the bimorph beams. The heaters cannot be integrated within the slender actuator beams for several reasons. The width of the beams must be minimized in order to achieve appreciable deflections from the bending moments. Such narrow beams do not provide enough sidewall protection for the polysilicon resistors, which would be exposed and etched away during the silicon etch step of the release process. In addition, long thin resistors with large resistance values would require voltages that exceed the CMOS process specifications. Furthermore, the piezoresistivity of the material would contribute additional nonlinearities upon deformation. In fact, the combination of thermal and piezoresistive effects in slender beams has been exploited to create self-sustaining mechanical oscillators [151]. Vertical actuators do not need to be narrow, since the bending moments generate deflections in the out-of-plane direction. Thus, a resistor 
embedded within the actuator would have sufficient sidewall protection and could possess a reasonable resistance value. Nevertheless, such a configuration would still suffer from piezoresistive nonlinearities.

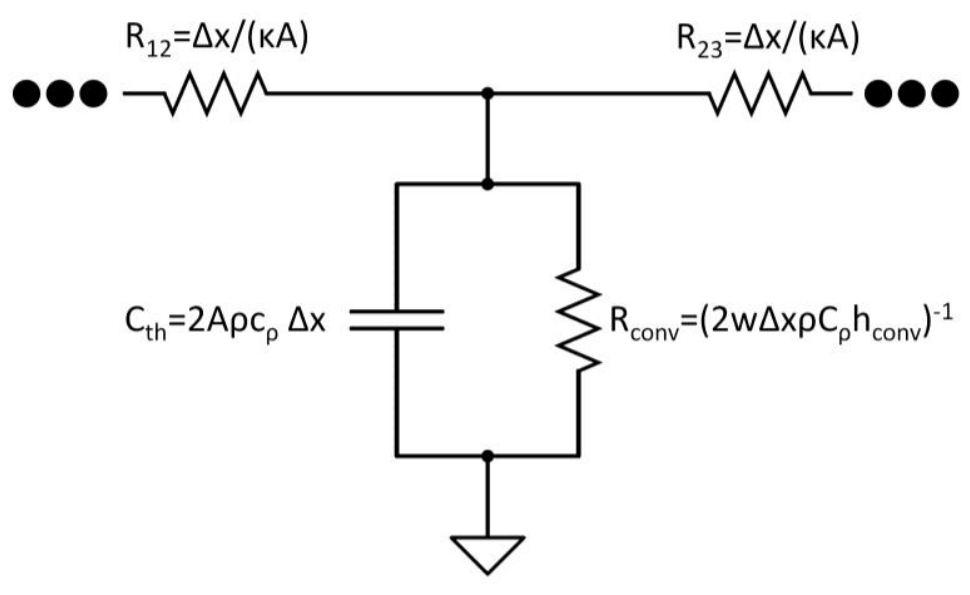

Figure 8: Thermal Domain Representation of a Lateral Electrothermal Actuator

\subsubsection{Mechanical Model}

The dynamic temperature distribution from the thermal model is coupled to the mechanical domain in order to calculate bending moments and deflection. The thermal dynamics are responsible for the lowfrequency roll-off that is observed in the transfer functions of these components. The actuators are intended to operate well below their mechanical resonant frequency to provide quasi-static displacements, so the dynamics in the mechanical domain are not considered in the model. For static mechanical analysis, the model of a lateral actuator consists of voltage sources representing the moments from thermal expansion and residual stress, and a spring (capacitor) that stores potential energy, as shown below in Figure 9.

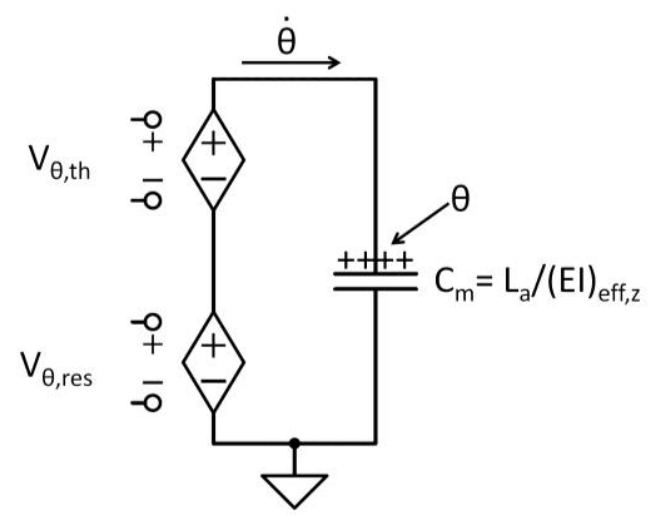

Figure 9: Mechanical Domain Representation of a Lateral Electrothermal Actuator 
The static Euler-Bernoulli beam equation is used to calculate the deflection in a beam under a constant moment load, as detailed in C.2. Under these conditions, which represent the simplest case, the value of the voltage source representing the moment from residual stress is:

$$
\begin{gathered}
V_{\text {res }, \theta}=M_{z, r e s} \\
V_{t h, \theta}=S_{M_{-} z, t h} \Delta T
\end{gathered}
$$

Where a scaling factor $S_{M_{z} z, t h}$ was introduced to express the voltage source in terms of temperature. The value of the capacitor that represents the flexural rigidity of the actuator beam is:

$$
C_{m}=\frac{1}{k}=\frac{L_{a}}{(E I)_{e f f, z}}
$$

If the deflection of the beam is required, the tip angle must be integrated. Since the force exerted by (or on) the actuator is at the tip, it is desirable to choose a spring constant and force source that reflect the end-point load geometry. This represents the second case. Values for the capacitors and voltage supplies are derived based on energy methods in order to properly represent the strain in the beam under a thermal moment, while referring the force to the tip. The new voltage sources are:

$$
\begin{gathered}
V_{z, r e s}=\frac{y_{\text {res }}}{C_{m, y}}=\frac{2 M_{z, r e s}}{L} \\
V_{z, t h}=\frac{y_{t h}}{C_{m, y}}=\frac{2 S_{M_{-z}, t h}}{L} \Delta T
\end{gathered}
$$

The new capacitor has a value of:

$$
C_{m, y}=\frac{L^{3}}{4(E I)_{e f f, z}}
$$

The model shown below in Figure 10 produces the correct displacement while appropriately scaling the moment such that the blocked force at the tip of the actuator is accurately represented by the new voltage source. 


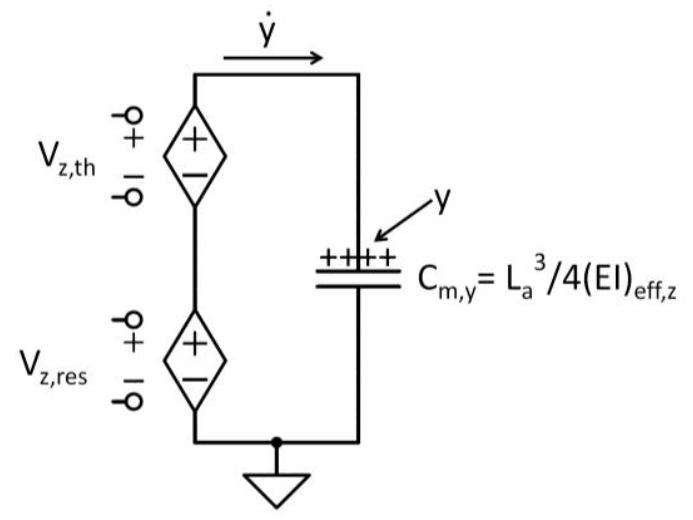

Figure 10: Mechanical Domain Representation of Electrothermal Actuator Deflection.

The difference between these models points out a subtle property of lateral electrothermal bimorph actuators. It is tempting to assume that if an actuator beam is loaded by a beam with the same geometry, its deflection will reduce by $50 \%$, since the load is matched to the source. However, this is only the case if the load is experiencing the same moment as the bimorph. If the load experiences a tipreferred force from a cantilever with the same geometry, then the bimorph will produce 4/7 of its initial displacement. This favorable scenario arises from the discrepancy between the apparent stiffness of the beam and the lower stiffness of a fixed-free cantilever, which is $3(E I)_{e f f, z} / L^{3}$. However, the situation is degraded when the symmetric configuration of 2 opposed bimorphs is adopted, as was done in this work. In this configuration, only $1 / 2$ of the strain energy in the beam is used to displace the load, since the other half of the beam is actuating in the opposite direction. The situation is exacerbated if the loading beam is in the guided-end configuration. This issue will be revisited in Chapter 9.

The third and most relevant case considers a temperature gradient over the length of the beam, which was shown to be an important effect in the FEA analysis of Figure 4. In this case, the thermal moment is a function of position, so the values of the capacitors and voltage sources are recalculated by direct integration of the beam equation in section C.2. The slope of the temperature between nodes of the thermal model is represented by:

$$
m_{t h}=\frac{\Delta T_{t i p}-\Delta T_{\text {base }}}{L}
$$

The new value for the tip angle is calculated by direct integration of the beam equation with a gradient in the moment load. Integrating once again yields the tip displacement. Energy methods are used to calculate the effective linear spring constant of the beam, which results in a capacitance of: 


$$
C_{y, m_{-} t h}=\frac{1}{k}=\frac{L^{3}}{4 E I}\left[\frac{1}{3}+\frac{L m_{t h}+2}{\left(L^{2} m_{t h}^{2}+3 L m_{t h}+3\right)}\right]
$$

The corresponding voltage source is:

$$
V_{t h, m_{-} t h}=\frac{q}{C}=2 S_{M_{-} z, t h} \frac{L^{2} m_{t h}{ }^{2}+3 L m_{t h}+3}{L\left(L m_{t h}+3\right)} \Delta T
$$

The complete quasi-static model of the electrothermal bimorph is shown in Figure 11 . The model accounts for thermal gradients and provides a realistic estimation of the tip-referred force and deflection by considering the strain energy produced by thermal bending moments. The quasi-static nature of the model means that it ignores the dynamics in the mechanical domain, which is why there is no lumped resistor to represent damping and no lumped capacitor to represent mass. The charge on the capacitor instantaneously follows the voltage on the source, scaled by a judiciously chosen compliance factor. Note that the resistor used to capture convection is tied directly to ground, since it is not affected by thermal isolation.

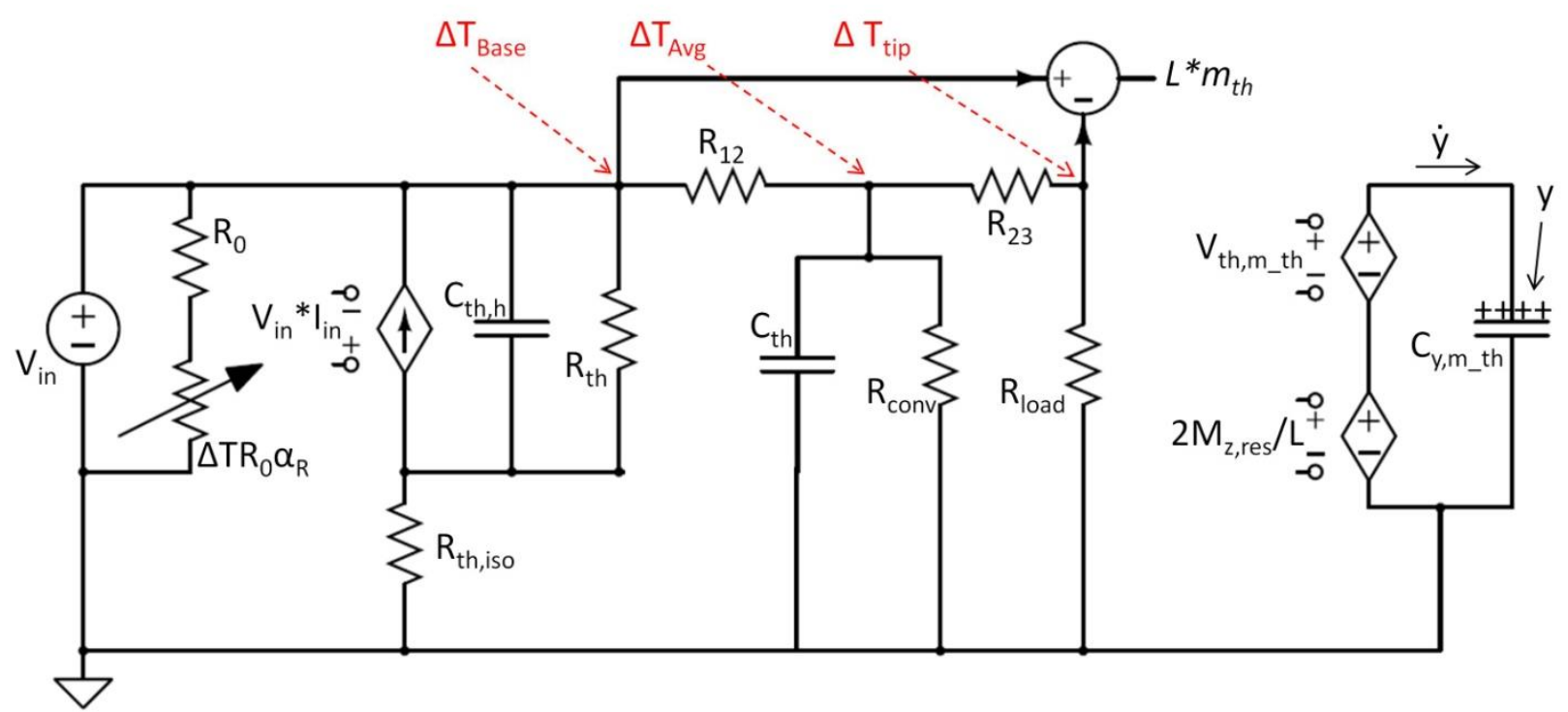

Figure 11: Electro-Thermo-Mechanical Model of Deflection

The test structure in Figure 12 is used to quantify the thermal isolation achieved by increasing the length of the thermal path between the heater and substrate. The static deflection of the bimorph beams is first captured in vacuum to quantify the thermal conductivity of the isolation chains materials. The figure shows the first set of vacuum measurements which was taken at a high power in order to obtain appreciable deflections to improve the measurement resolution. Unfortunately, at these high 
temperatures and stresses, the bimorphs plastically deform and do not return to their original positions. Note that the bimorph without thermal isolation does not experience this deformation, because it does not reach a high enough temperature and deflection. Because of this effect, the increase in isolation chains does not seem to produce a linear increase in deflection. At lower voltages, with a new test structure, a linear increase was observed. With this data, the model is validated without convective effects. An FEA model that captures the temperature distribution in thermal isolation chains of various lengths will be discussed in section 3.4.

\section{Deflection of Thermally Isolated Bimorphs in Vacuum}
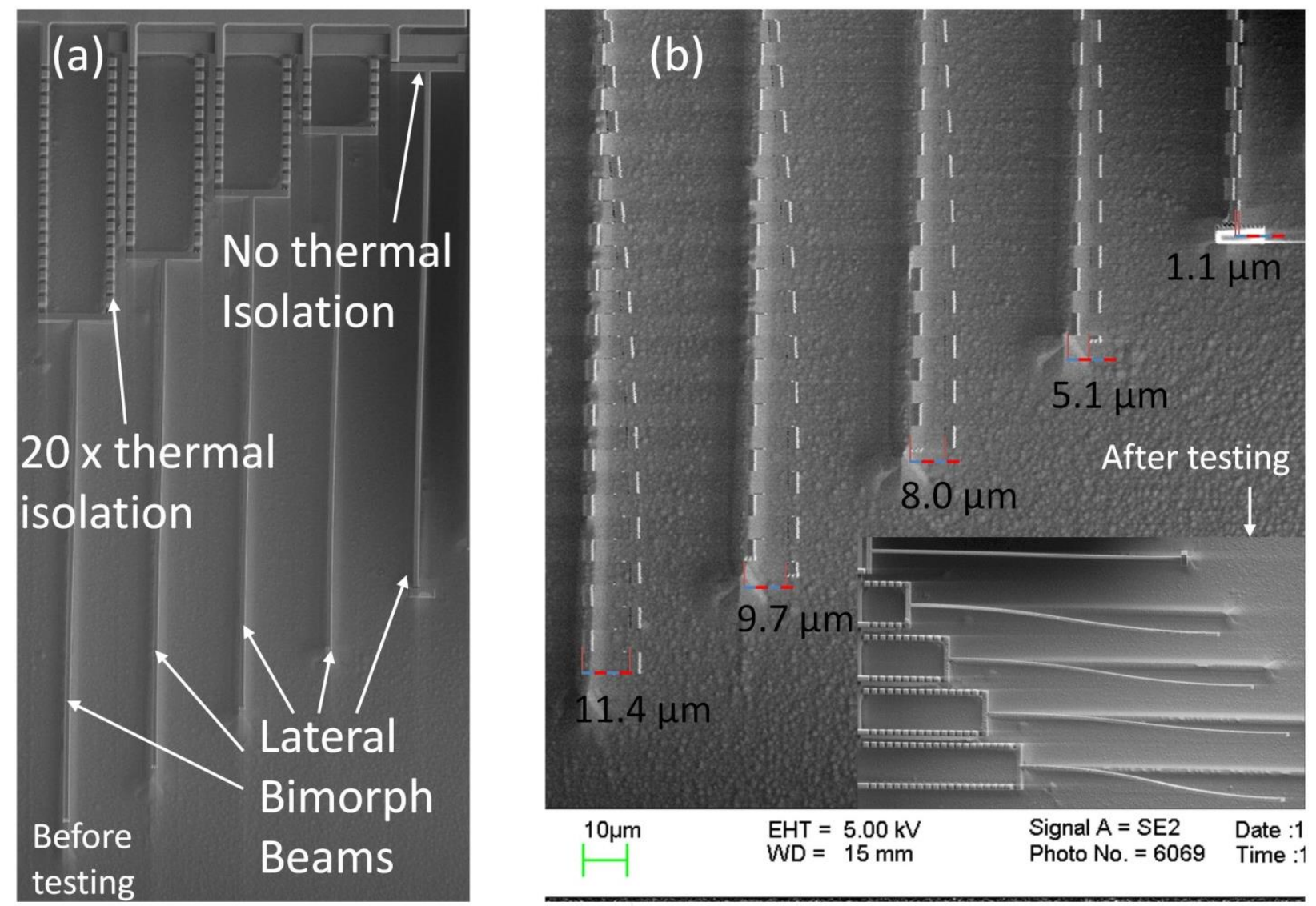

Figure 12: Deflection of Thermally Isolated Bimorphs in Vacuum. (a) Test structure before actuation. (b) Test structure under an applied square wave voltage. Inset: Test structure after thermally induced plastic deformation.

Next, static deflection data is obtained over a range of voltages in air. These results are used to quantify the convective heat transfer quantities. Data was captured with the same devices using a stroboscopic motion capture system, and is presented below in Figure 13. After 10 thermal isolation links, convective 
losses begin to dominate and establish a point of diminishing returns - the addition of more isolation at this point is futile. Note that in vacuum, since there are only conductive losses, such a point of diminishing returns does not exist.

\section{Thermal Isolation in Air}
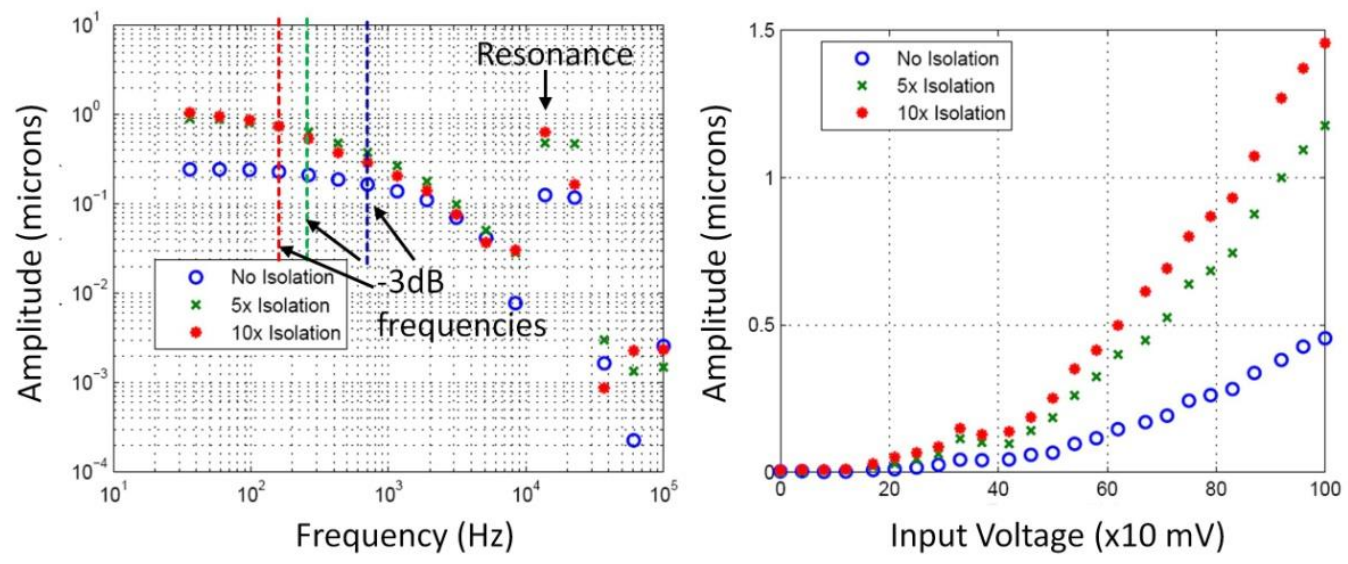

Figure 13: Frequency Response (left) and Static Deflection (right) of Thermally Isolated Bimorphs in Air

Finally, a dynamic frequency sweep is performed to capture the locations of the poles in the thermal RC transfer function. These values are used to set the thermal capacitances in the model. The static model eventually fails when mechanical dynamics come into play at high frequencies, where resonance is clearly observed.

In this section, it has been shown that the mechanical deflection of thermally actuated beams is linear in proportion to temperature. In fact, even when there is a thermal gradient along the length of the beam, the deflection at the tip is linear with the temperature and the thermal gradient. It is therefore important to seek a driving scenario in which the temperature can be controlled in a linear fashion, in order to guarantee the controllability of the actuators. A commercially available precision IC multiplier (AD532) was configured to take the square root of an applied input voltage, then apply it to the actuator. The linearization of the output position with respect to the input voltage is demonstrated by measurements taken in a scanning electron microscope (SEM), shown in Figure 14. A high-contrast image of the edge of the actuator was first obtained, and then aligned with the slow-scan direction of the SEM. Upon actuation, the deflection of the actuator was observed in direction of the SEM's fastscan axis. In order to capture quasi-static behaviour, the SEM scan rate was set to 30 seconds, and the actuator input signal was chosen with a period of 10 seconds. 

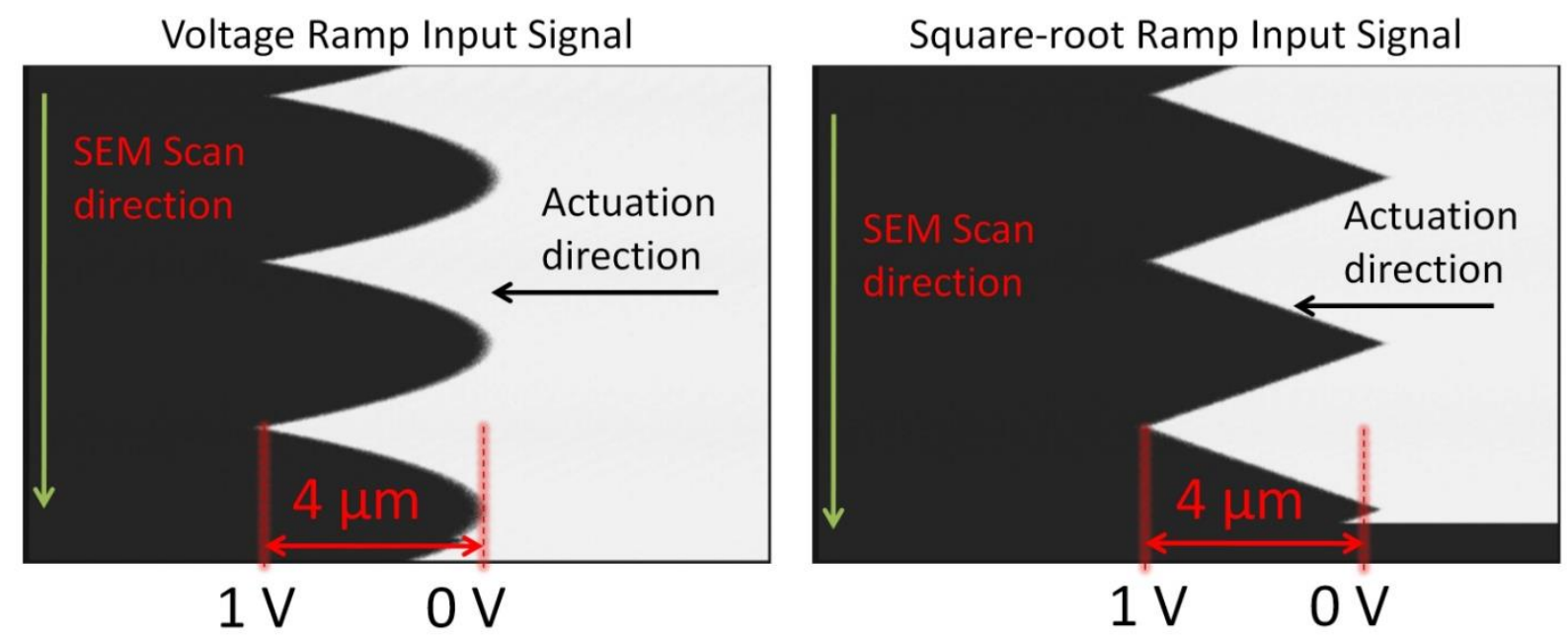

Figure 14: Scan Linearization with Square-Root Circuit. SEM image corresponds to a slow scan in the direction orthogonal to actuation.

\subsubsection{Flexural suspensions}

Flexures are used throughout the design of CMOS-MEMS SPM's to connect components to one another mechanically. In the electrical domain, flexures may be used to carry signals between components. Thermal coupling between components is an unavoidable consequence when the flexures are used for electrical routing. The design of compliant structures is an area of significant interest in the MEMS community, and there are methods to optimize the shape of flexures for mechanical or geometric advantage, or for a desired motion path [152], [153].

In this work, the design constraints on flexures are simple: for example, in-plane flexures are intended to be rigid in the direction of applied force, and compliant in the orthogonal direction. In addition, they are constrained by the overall stiffness budget of the instrument. Furthermore, Manhattan-style geometries are generally enforced in the design-rule-checking process by the CMOS foundries. To circumvent this restriction for simple beam geometries, a Cadence script was written to generate beams with arbitrary lengths and angles that comply with the design rules. Thus, the range of flexure designs is limited to:

1. Guided-end beams that are used to provide high stiffness and high compliance in orthogonal directions. A crab spring is a good example of such a flexure mechanism.

2. Serpentine and spiral designs are used to provide rigidity in a linear direction and compliance in a rotary direction. These flexures also provide motion amplification, but there is a linear tradeoff between mechanical advantage and geometric advantage. 
3. Chevron designs are used to provide rectilinear motion amplification. These designs also involve a direct trade-off between mechanical and geometric advantage.

\subsubsection{Electrical Domain}

Several electrical routing paths may be incorporated into a single flexure to enable the interconnection of sensors and resistive heaters throughout the instrument, and to provide a signal path to the tip. At low frequencies, the electrical path is simply modeled as a series resistor. Although the CMOS interconnect Aluminum has excellent conductivity, the flexure geometry is typically long and thin, resulting in a series resistance that cannot be ignored for sensor applications. The resistance should also be considered when large currents are routed through flexures to power actuators. For example, in one design, a low resistance $(<100 \Omega)$ vertical actuator requires $5 \mathrm{~mA}$ routed through a long, thin $(300 \mu \mathrm{m}$ $\mathrm{x} 2 \mu \mathrm{m}$ ) flexure. This is a violation of the foundry design rules, and its severity is increased by the fact that the beam is thermally isolated from the substrate. The self-heating in the flexure causes plastic deformation in the metal of the flexures at high temperatures and strains, resulting in permanent deformation of the device [154]. To lower this resistance, one may use all of the metal layers in parallel to route critical signals. In addition, for differential signals that are intended to operate in a Wheatstone bridge configuration, the series resistance should be matched. The problematic nature of series resistance in the shared ground path of sensors will be encountered in section 0 which discusses "ground bounce."

The situation is exacerbated by the presence of leakage currents that are captured in the model by a shunt resistor. The leakage currents are particularly problematic when the tip-sample interaction signal is a low current signal, as in the case of STM or in the characterization of nano-scale electronic devices. The use of a triax configuration or driven shield configuration is common practise in these scenarios.

At moderate frequencies, a signal routed through a flexure may experience capacitive coupling to the ground plane on the top and/or bottom metal layers. A measured bode plot reveals the effect of this impedance on the transfer function at high frequencies. This situation introduces challenges in the measurement of small AC signals, such as those encountered in KPFM. The triax method may be used here, but only up to the bandwidth of the driver circuit, which is inversely proportional to its transimpedance gain - in other words, low signal currents are associated with low bandwidths. The resonant frequency of the KPFM may be lowered to remedy this symptom, but the imaging bandwidth will suffer as a consequence. 
Compliant flexures are used to route a microwave signal to the tip of the SMM instrument. At frequencies of $3-20 \mathrm{GHz}$, the impedance of the transmission line may be controlled to $50 \Omega$ to match the impedance of the rest of the system, thereby preventing energy loss through reflections which would occur at points of impedance mismatch. A micro-coaxial design provides controlled impedance and prevents capacitive coupling of the propagating signal to the sample surface. Using microwave transmission line test structures, it was determined that for the short distances of propagation encountered in the SMM, impedance matching is less of a concern than capacitive coupling to the substrate. Of greater importance are the series resistance of the transmission line, which should be minimized in order to conserve the reflected power from the tip-sample region, and the capacitance to ground. The mechanical requirements of the flexure's compliance (i.e. thin and long) are therefore at odds with the desire for a low series resistance (i.e. wide and short). In addition, it may be desirable to use only the top and bottom metal layers for routing, in order to decrease the capacitance to ground. The model in Figure 15 may be used to represent the routing of electrical signals through a flexure up to $\mathrm{GHz}$ frequencies, when impedance matching is not a concern.

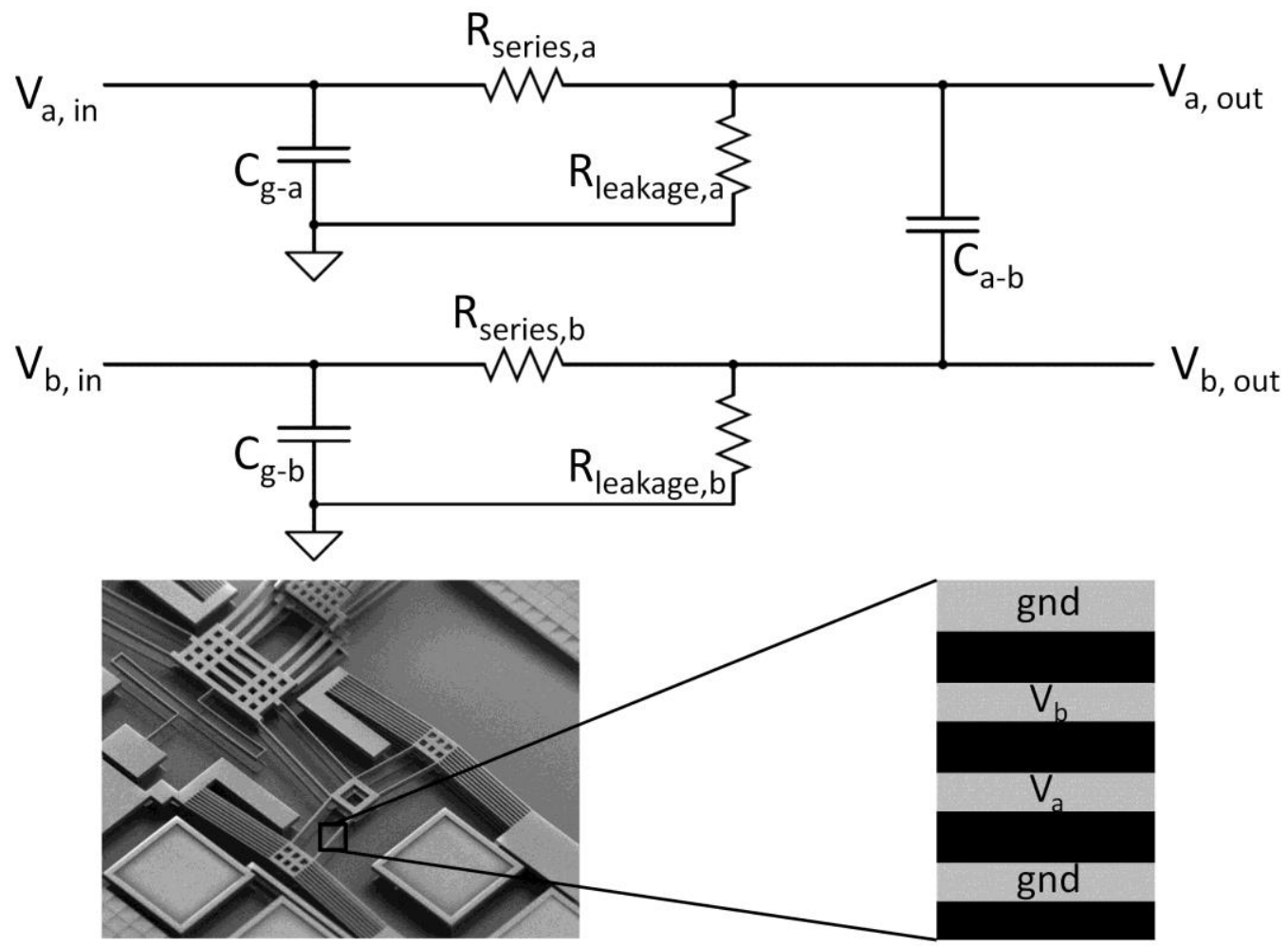

Figure 15: Electrical Model of Routing in Flexures 


\subsubsection{Thermal Domain}

The mechanical design of flexures favors long and slender beams to reduce the loading on actuators. These components are considered to be "thermally long" as well, so the high frequency actuation of resonant cantilevers does not result in the transmission of AC heat through them. Instead, each end of the flexure assumes a temperature that is equal to the sum of the quasi-static temperature (from low frequency lateral actuation) and the RMS temperature from high frequency actuation. The beams present a thermally conductive path between interconnected devices, so the model must capture this DC and quasi-static heat flow from end-to-end. The model from section 3.3.2 may be used, with a single capacitor to capture the small heat capacity of the beam and two resistors to capture the thermal conductance. The value of the capacitor is:

$$
C_{t}=2 A \rho c_{p} L
$$

The value of the resistors is:

$$
R_{12}=R_{23}=R_{\text {Cond }}=L / \kappa A
$$

The effective density, specific heat capacity, and thermal conductivity of the beam are calculated from equations (10.59) and (10.60). The cross section of a flexure is typically simpler than that of a lateral bimorph, since the internal metal layers are not offset. The number of members in the equations is therefore reduced to a maximum of 8 , as shown in Figure 15 above, from the electrical model.

\subsubsection{Mechanical Domain - Orthogonal Scanners}

The mechanical model of a flexure must capture its quasi-static deflection and the loading force that it applies. The three flexural suspensions designs that are used in this work are orthogonal suspensions, cylindrical suspensions, and chevron suspensions.

An XY stage that employs guided-end flexures to decouple orthogonal in-plane actuators is shown in Figure 16, along with its mechanical lumped element model. The loading effect of the flexures is captured by a capacitor that is placed in series with the actuator's mechanical capacitor. It should be noted that the flexure that is physically connected to the $x$-direction actuator (the $x$-flexure) presents a load to the y-direction actuator, and vice versa. The deflection of the shuttle is the same as the deflection of the actuators since the flexures are rigid in their axial directions. The out-of-plane compliance of the flexures detracts from the overall vertical stiffness of the device. In the Z-direction, 
the mechanical path from the tip to the substrate consists of 2 identical flexure springs and 2 identical actuator springs, which may be modeled as a combination of 3 springs for convenience. The two flexure springs are combined into a single capacitor, which is connected in series with the actuator capacitors.

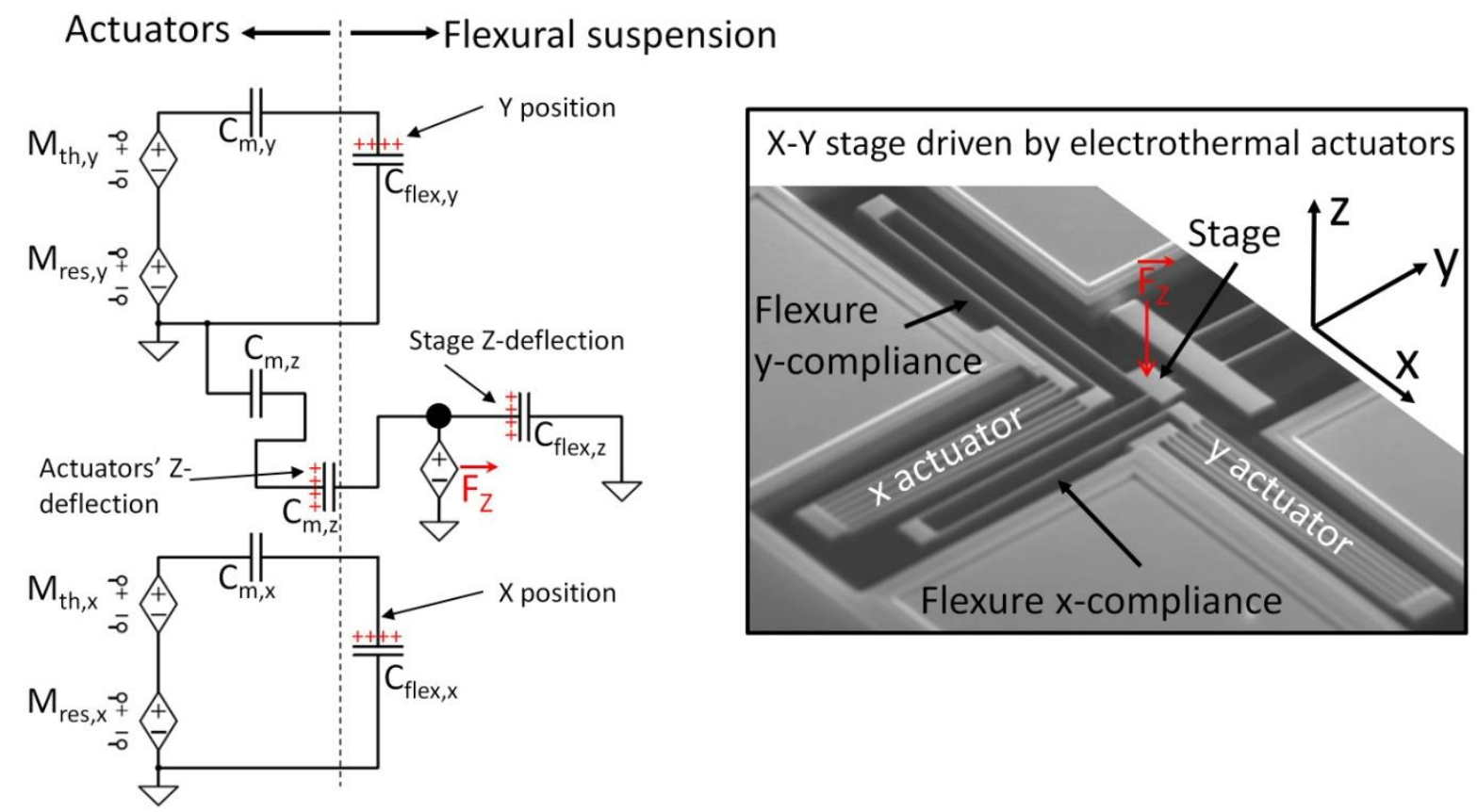

Figure 16: Mechanical Model of X-Y Flexural Suspension

Since the beams are folded into a U-shape, axial loading can be neglected. The out-of-plane stiffness of the flexural suspension, from the stage to the connection points on the actuators, may be modeled as the parallel combination of 2 pairs of series-connected guided-end beams. The capacitor has a value of:

$$
k_{z}=\frac{E w t^{3}}{L^{3}}, \quad C_{z}=\frac{L^{3}}{E w t^{3}}
$$

And the in-plane stiffness and capacitance values are:

$$
k_{x, y}=2 \frac{E w^{3} t}{4 L^{3}}, \quad C_{x, y}=\frac{2 L^{3}}{E w^{3} t}
$$

The trade-off that must be considered in the design of orthogonal guided end suspensions is that the out-of-plane stiffness and in-plane stiffness are linearly related, for a given beam cross-section. Thus decreasing the length of the flexure will increase the out-of-plane stiffness at the expense of adding a load to the lateral actuator, reducing the scan-range of the instrument. The beam should have a high aspect-ratio to minimize out-of-plane buckling and to improve the linearity of its in-plane stiffness. 


\subsubsection{Mechanical Domain - Cylindrical Coordinate Scanners}

An SPM that employs a suspension for cylindrical coordinate scanning is shown in Figure 17. Actuators are placed in parallel such that the tip motion can be controlled in 3 degrees of freedom. Commonmode actuation results in tip displacements in the radial direction $r$. Differential actuation results in tip displacements in the angular direction $\theta$. A coordinate transformation is used to convert the rectilinear displacements of the in-plane actuators to the cylindrical motion of the tip. The vertical actuator in this design makes use of a design pattern similar to lateral electrothermal actuators in order to cancel the tip-rotation so that the motion of the tip is in the z-direction.

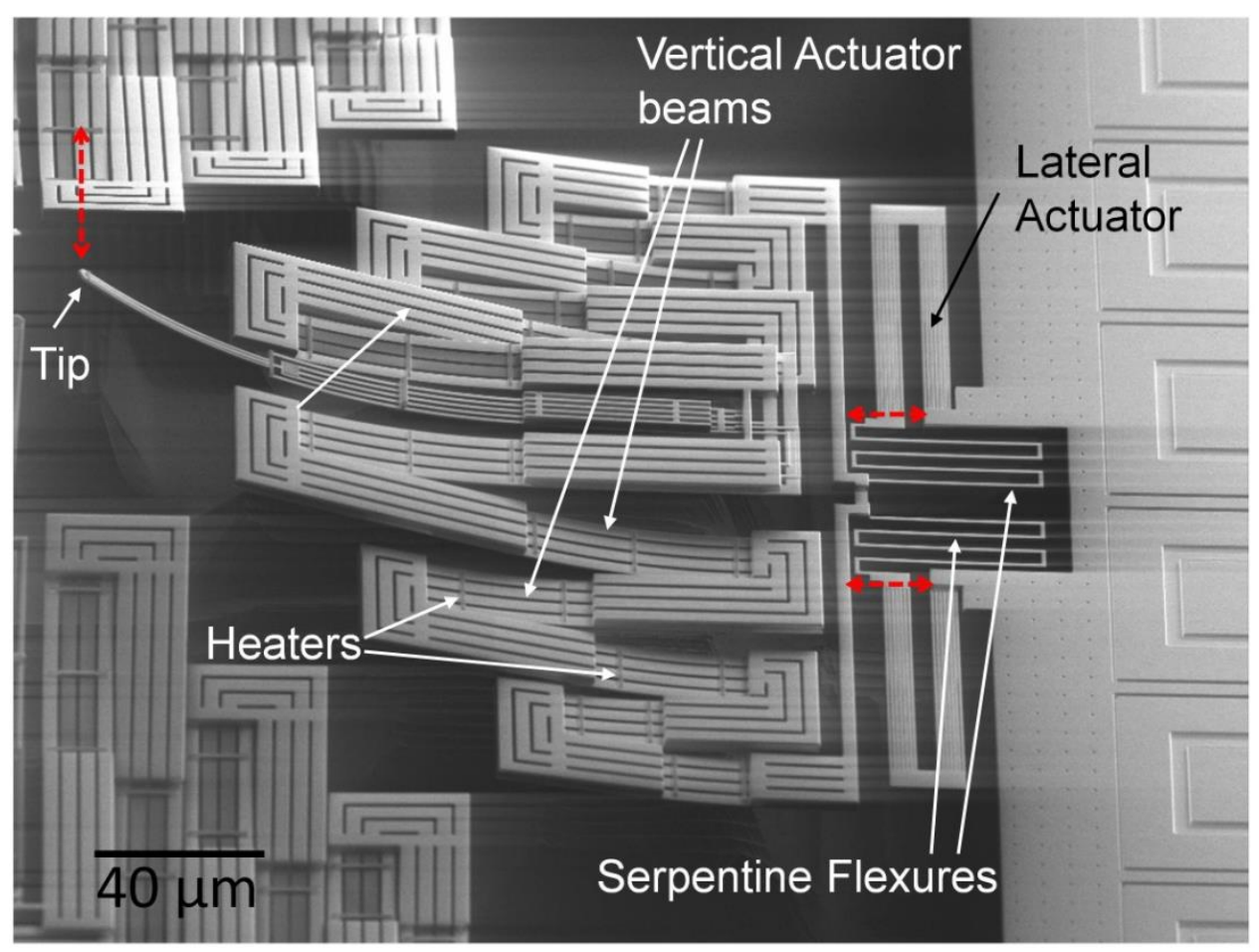

Figure 17: Flexural Suspension for Cylindrical Coordinate Scanning

The serpentine spring in a cylindrical suspension is responsible to converting the linear motion of the actuators into rotation about the z-axis of the chip. The spring should be designed to have low torsional rigidity to minimize the load to the lateral actuators, and to reduce the mechanical coupling between them. In addition, the tip-referred out-of-plane stiffness should not be overly compromised. In other words, the torsion spring should be compliant to in-plane torque, but stiff to out-of-plane torque. These designs are subject similar stiffness-to-range tradeoffs as other flexural suspensions. The device in Figure 17 above has a lateral range of $120 \mu \mathrm{m}$ and a vertical range of $>50 \mu \mathrm{m}$, but it is too compliant to use in the SPM modes discussed here. 
A simple geometric model to calculate the position of the tip based on the in-plane deflections of the lateral actuators is:

$$
\theta=\tan ^{-1} \frac{y_{2}-y_{1}}{d_{12}}, \quad r=L_{t-b}+\frac{y_{2}+y_{1}}{2}
$$

where $d_{12}$ is the distance between the actuators and $L_{t-b}$ is the length from the tip to the base of the zactuator. This model is valid for small deflections and a large separation between flexures. In reality, the linear motion of the actuator gives rise to a reaction force and a torque at the base of the torsion springs, so one must balance the force and moment equations. FEA is used to determine the effective spring constants for various geometries, as shown in Figure 18.
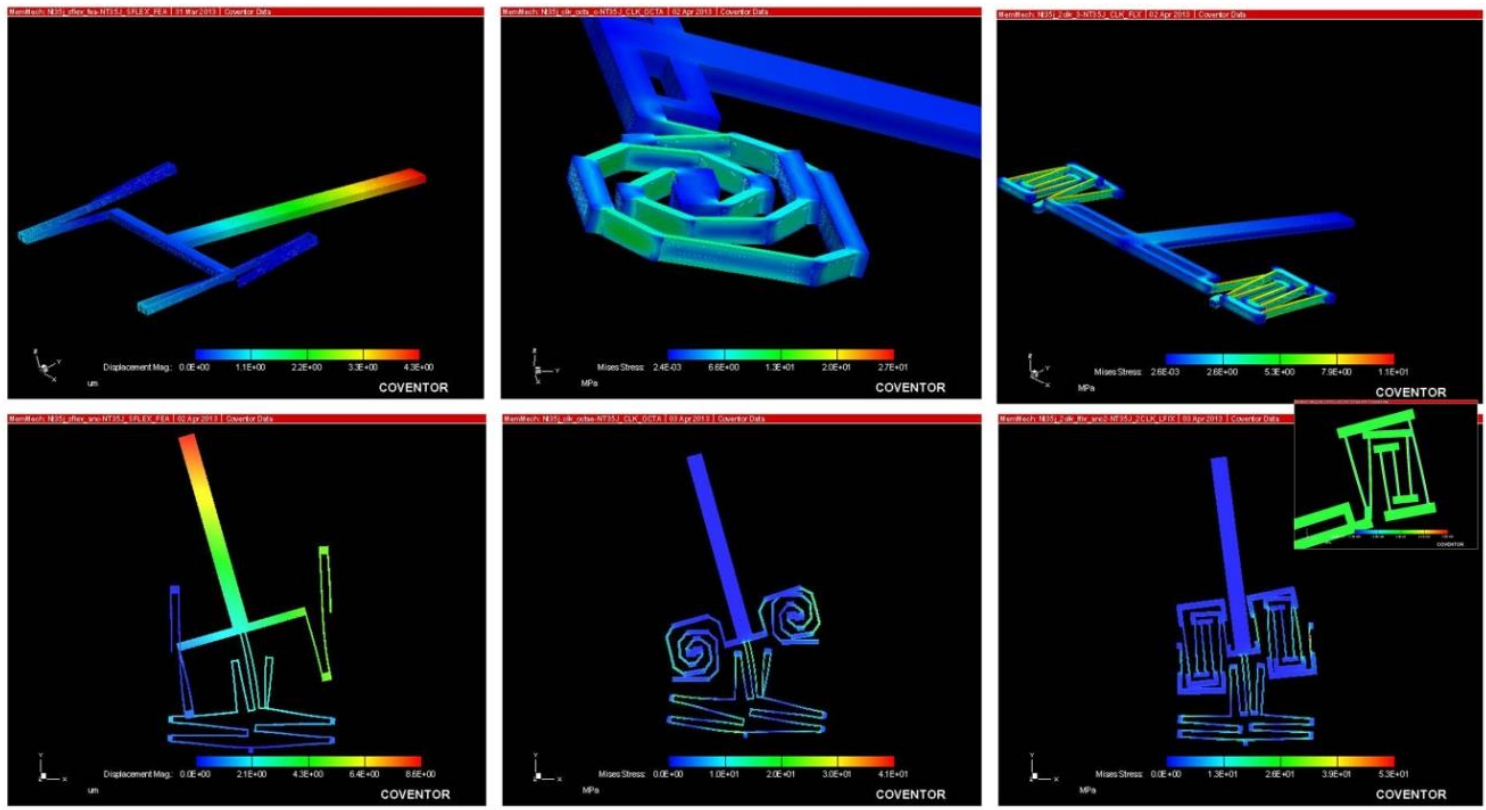

Figure 18: FEA of Cylindrical Scanner Flexural Suspensions. Top Left: S-shaped springs are compliant in the Z-direction. Center: Torsional spring is compliant due to angled segments. Right: Improved suspension design.

The lumped element model for cylindrical scan flexural suspensions (Figure 19) considers the mechanical coupling between the actuators that is caused by the torsion springs. In the model it is assumed that the left actuator exerts a torque on the right spring, and vice versa. The two serpentine flexures are lumped into a single torsion spring that is modeled with a capacitor that stores strain energy upon bending. The charge on this capacitor represents the angular deflection of the tip. The model reflects the fact that the torsion springs experience opposite bending moments from the left and right actuators, by choosing different polarities for the voltage sources. The thermal moment in the actuators 
must now be shared between the flexural rigidity of the actuator beams, the torsional stiffness of the suspension, and the compliance of the opposite actuator due to coupling through the flexures. When one actuator is fully extended, it "pulls" the other actuator in the same direction, potentially causing unwanted distortions in the scan pattern of the tip. An effective way to reduce the coupling is to increase the distance $d_{12}$ between flexures; however, this reduces the scan range in the $\theta$ direction, as indicated by equation (3.24). This loading is reflected in the wiring of the model. When both actuators are actuated by the same amount, there is no loading, and thus no charge on the capacitor that represents the torque spring.

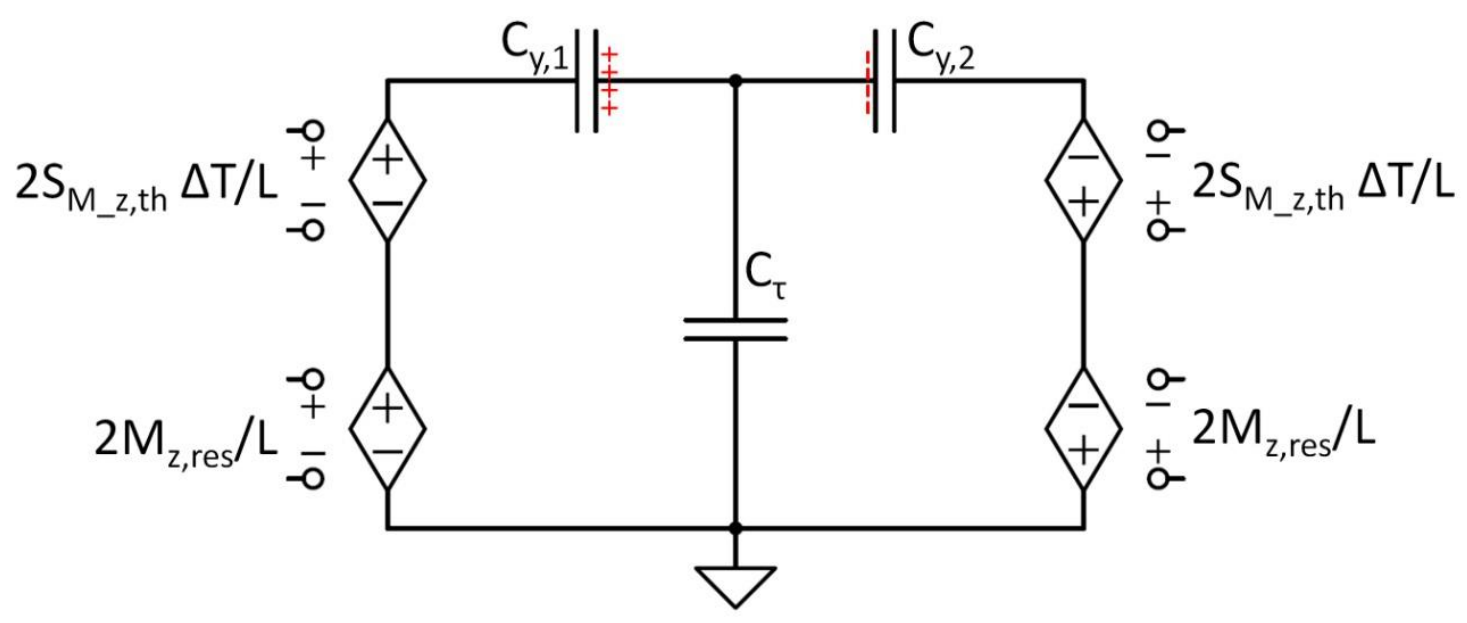

Figure 19: Mechanical Model of Cylindrical Scanner Flexural Suspension

The torsional rigidity of the suspension is first converted to a linear spring constant. For small angles, the angular deflection of the spring is:

$$
\frac{F d_{12}}{k_{\theta}}=\theta
$$

Where $k_{\theta}$ is the torsion spring constant. The linear restoring force from the torsion spring may be expressed in terms of geometric parameters. Under the small angle approximation,

$$
\theta d_{12} \approx y_{2}-y_{1}
$$


The effective linear spring constant and capacitance are:

$$
k_{e f f, y}=\frac{k_{\theta}}{{d_{12}}^{2}} \quad C_{\tau}=\frac{d_{12}^{2}}{k_{\theta}}
$$

Flexures may also be used to provide a geometric or mechanical advantage. The chevron or "saggital spring" suspension shown in Figure 20 is used to provide a geometric advantage to amplify the motion of a chevron actuator.

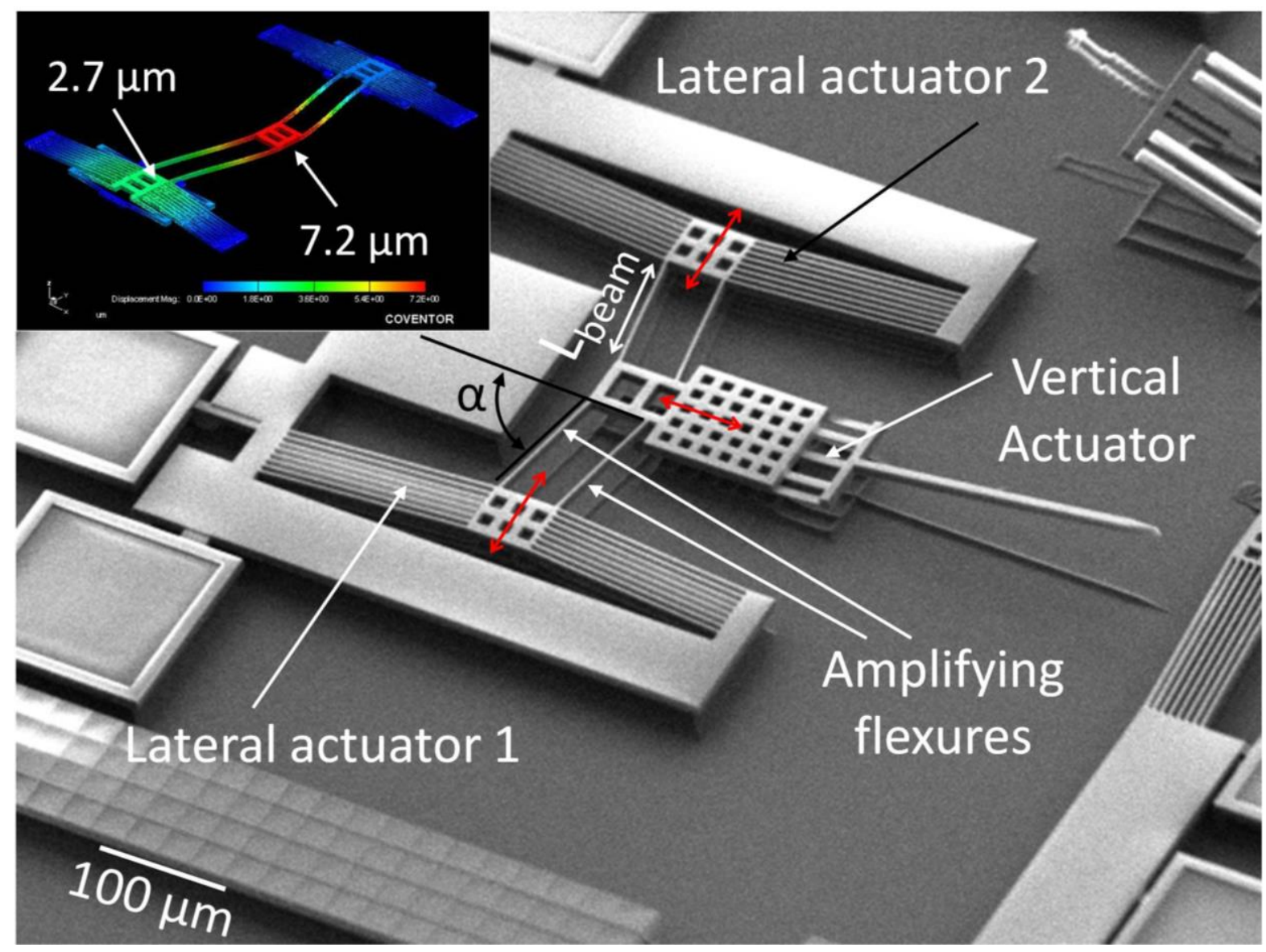

Figure 20: Motion Amplifying Chevron Suspension

The stiffness of such a structure in the direction of the actuator represents its load capacitance [155]:

$$
K_{x}=\frac{12 E I_{z}}{l_{\text {beam }}^{3} \cos ^{2} \alpha}
$$


The stiffness in the orthogonal direction is:

$$
K_{y}=\frac{12 E I_{z}}{l_{2}^{3} \sin ^{2} \alpha}
$$

These expressions consider bending only. The geometric advantage, or gain of the structure can be calculated by equating the potential energies in both directions and solving for the ratio of deflections:

$$
\frac{1}{2} K_{x} x^{2}=\frac{1}{2} K_{y} y^{2} \quad \therefore \quad \frac{y}{x}=\frac{\sin \alpha}{\cos \alpha}=\tan \alpha
$$

In other words, for an input in the $x$ direction of $1 \mu \mathrm{m}$, the output deflection in the $y$ direction is $\tan \alpha$ $\mu \mathrm{m}$. The lumped element model of a motion amplification flexure includes a capacitor $C_{x}$ placed in series with the actuator's compliance to capture the loading effect on the actuator. A second capacitor $C_{y}$ may be used to capture the output-referred stiffness of the device. The values of the corresponding gain and capacitances are:

$$
A=\tan \alpha \quad C_{x}=\frac{1}{K_{x}} \quad C_{y}=\frac{1}{K_{y}}
$$

\subsection{Thermal Shunt Paths and Thermal Isolation Paths}

Chip-scaled SPM's have many localized heat sources that must operate independently in order to scan the tip, and to measure tip-sample thermal interactions. In addition, many of the sensors distributed throughout the structure and at the tip are sensitive to temperature. Therefore, thermal management is of great importance.

A thermal shunt path should provide a short thermal path to the substrate, which acts as a heat sink. This way, heat is redirected to the substrate instead of to other parts of the SPM. The mechanical loading of the element should be minimized, and its electrical conductivity can be used for signal routing.

Thermal isolation paths provide a robust mechanical connection between parts, with a high resistance in the thermal domain. In many cases, an electrical path is required as well. One example of such a structure is shown in Figure 21, where a temperature sensor is intended to measure the heat transfer in the tip-sample region. In this case, the heat flow to the sample should dominate over heat flow to the rest of the SPM. 


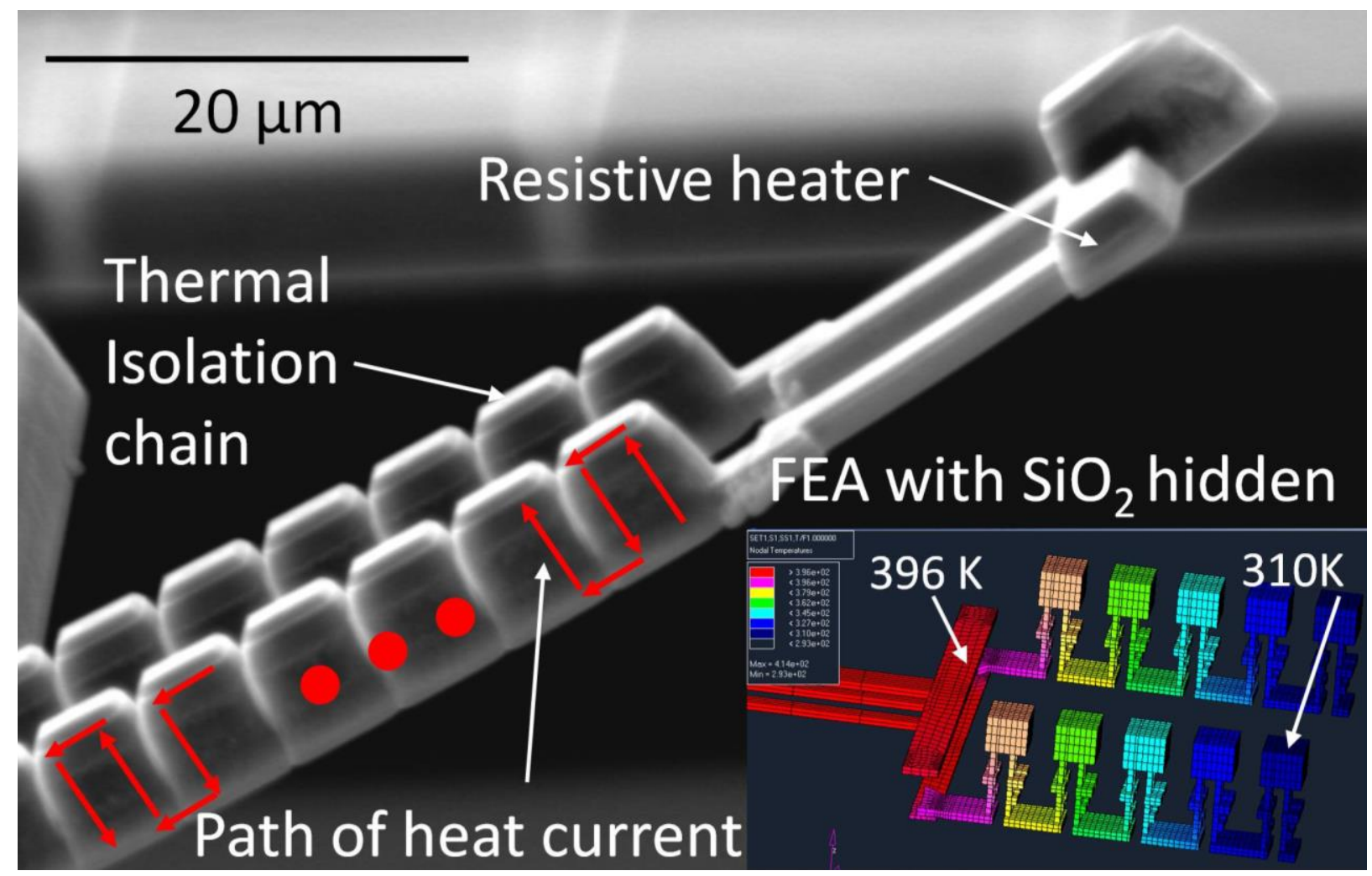

Figure 21: Thermally Isolated Tip.

In SThM, the dynamics of the thermal shunt path become important. The phase and amplitude of the temperature at the tip location must be obtained in order to extract information about the local thermal conductivity and heat capacity in the tip-sample region.

\subsubsection{Electrical Domain}

The electrical domain representation for a thermal shunt path is a single resistor. Only the low frequency and moderate frequency behaviour need to be captured, since these elements are not used to carry high frequency signals.

In order to increase the thermal resistance in the device of Figure 21, a long and tortuous path is introduced between the ends of the structure. Heat and electrical current are forced to flow through tungsten vias that have small cross-sections of $\sim 450 \mathrm{~nm} x \sim 450 \mathrm{~nm}$. The electrical resistance per via and the sheet resistance of the metal layers are provided by the foundry and incorporated into the lumped electrical resistance in the model: 


$$
R_{\text {elec }}=N R_{\text {via }}+\sigma_{\text {elec }, A l} \sum_{\text {segments }} \frac{L_{i}}{A_{i}}
$$

When evaluating the summation, the lengths $L_{i}$ and cross-sectional areas $A_{i}$ are taken with respect to the direction of current flow.

\subsubsection{Thermal Domain}

Shunt paths are represented in the same way as flexures in the thermal domain. Isolation paths, however, must include the additional thermal resistance introduced by the Tungsten $(w)$ vias which have lower thermal conductivity. In the DC temperature distribution of a thermal isolation path, most of the thermal gradient occurs along the length of the vias, since they are narrow and have a high aspect ratio. This can be observed in the FEA results from Figure 21, since there is a noticeable temperature difference between the top and bottom of the unit cell, but not along its length. If electrical routing is not required, a purely mechanical thermal isolation path may be used by removing the vias to force heat to flow through the $\mathrm{SiO}_{2}$. In Figure 22 it is shown that heat flux through the Aluminum layers is $\sim 5 \mathrm{X}$ higher than conduction through the $\mathrm{SiO}_{2}$. The device has a $150 \mathrm{~K}$ temperature difference between the heater and anchor.
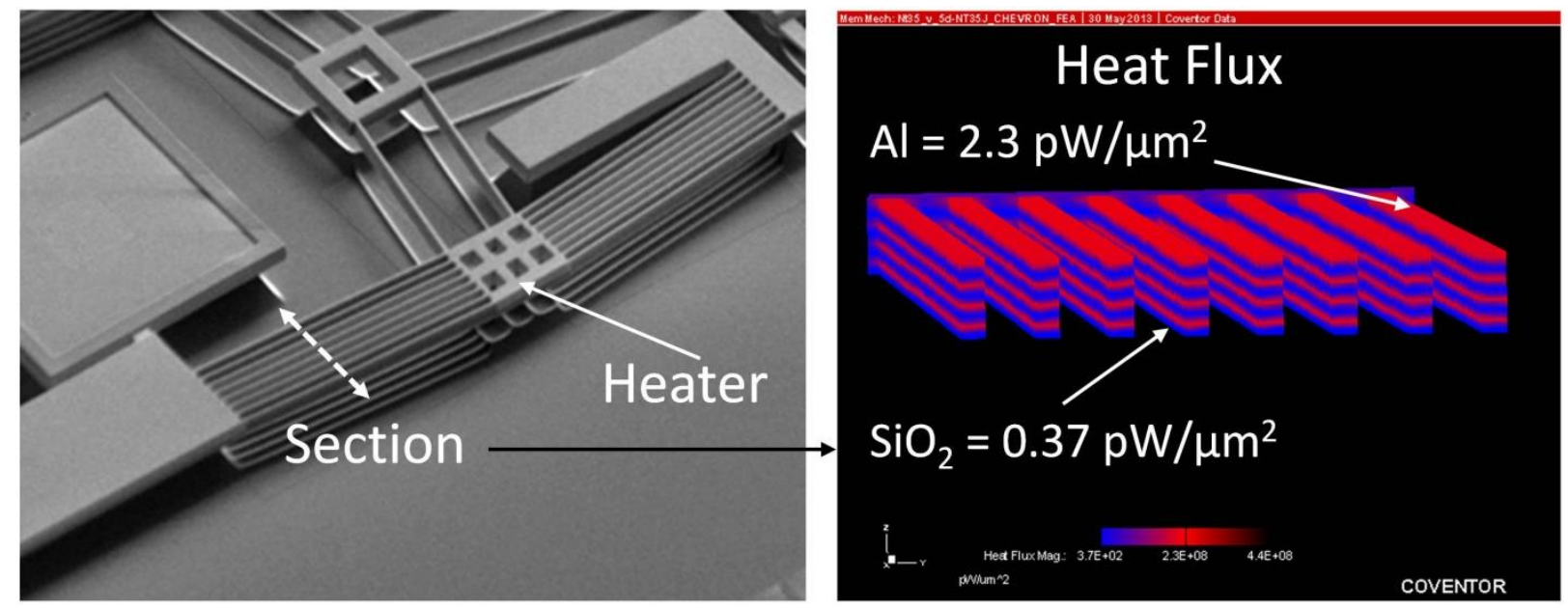

Figure 22: Thermal Conduction in a Composite Beam

When vias must be used for electrical routing, each unit in the thermal isolation chain is treated as a lumped model with the representation derived in section 3.3.2. The series resistance of each unit is: 


$$
R_{t h}=N \kappa_{w} \frac{L}{A}+\kappa_{A l} \sum_{N+2} \frac{L_{i}}{A_{i}}
$$

When evaluating the summation, the lengths $L_{i}$ and cross-sectional areas $A_{i}$ are taken with respect to the direction of heat flow. The thermal conduction of $\mathrm{SiO}_{2}$ is neglected. The thermal capacitance for a unit is evaluated by:

$$
C_{t h}=N\left(\rho c_{p}\right)_{w} V_{v i a}+\sum_{\text {segments }}\left(\rho c_{p}\right)_{i} V_{i}
$$

where $N$ is the number of vias, and the summation is over the Aluminum and $\mathrm{SiO}_{2}$ segments in the unit. Since these structures have more surface area than a monolithic beam, the resistor that captures convection considers the vertical surfaces:

$$
R_{\text {conv }}=\frac{T_{a}-T_{2}}{i_{\text {conv }}}=\frac{1}{2 w \rho c_{p} h_{\text {conv }}\left[L_{\text {unit }}+t_{\text {unit }}\right]}
$$

where $t_{\text {unit }}$ represents the total thickness of the beam. A model for a chain of 3 elements is shown in Figure 23.

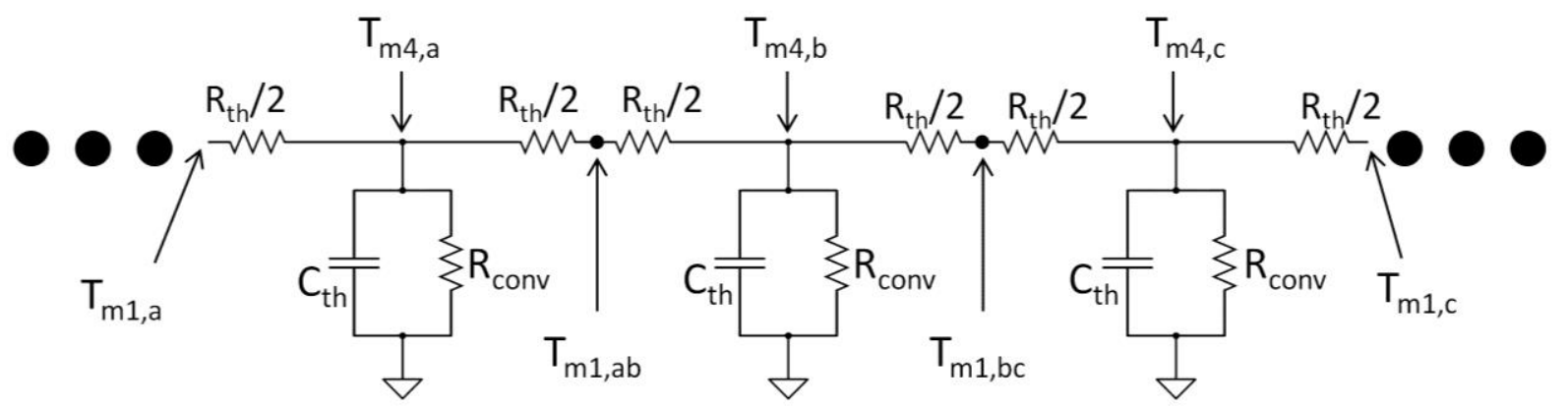

Figure 23: Thermal Domain Model of a Thermal Isolation Chain

A transient simulation showing the temperature at 5 different elements of the thermal isolation chain is shown in Figure 24. This plot reveals the amplitude and phase dependence of temperature along the beam, showing that the amplitude decays and the phase lags along the length of the beam as expected. 


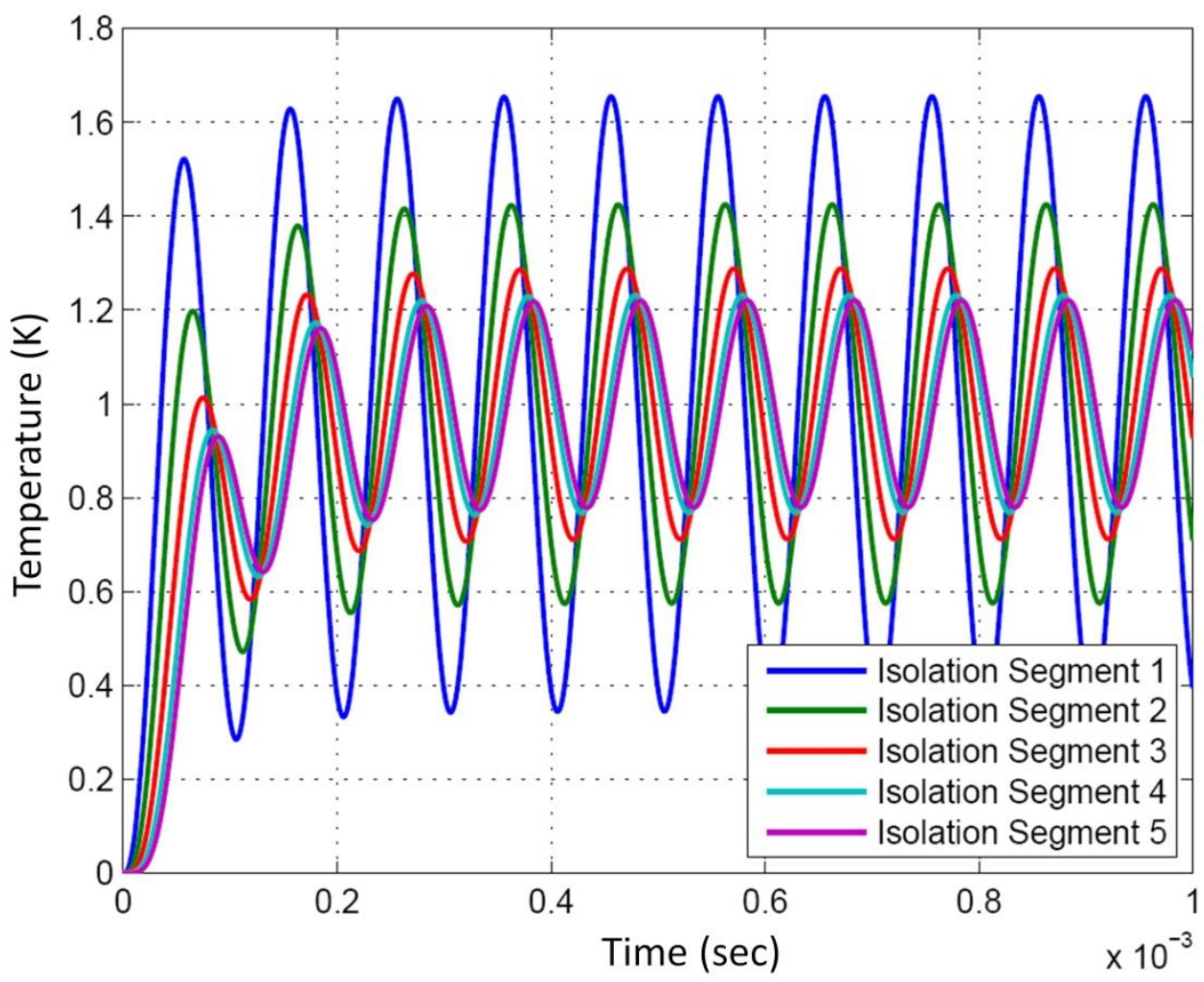

Figure 24: Amplitude and Phase Dependence of Temperature in a Thermal Isolation Chain

\subsubsection{Thermal Transmission Line Model}

Lumped parameter models like the one presented above are useful when the wavelength of interest is larger than the geometry being considered. As the frequency of the thermal excitation source is increased, the full solution to the heat equation shows that the AC temperature has an exponential dependence on position, as well as a phase dependence. To capture this behaviour, a distributed parameter model should be developed. The methodology shown here is borrowed from the concept of transmission lines in the field of microwave engineering. The lumped parameter models for a microwave transmission line and the thermal isolation path are the same, except for the additional series inductance of the microwave transmission line. Details on the derivation of the distributed parameter model are included in section C.3.

The model begins with a section of the thermal conduction path with the values of the components scaled to match its length, as shown in Figure 25 below. After Kirchoff's Voltage law is applied to this model, one takes the limit as the length of the section approaches zero. The value for the characteristic impedance of the line is: 


$$
Z_{0}=\frac{V_{0}^{+}}{I_{0}^{+}}=\frac{V_{0}^{-}}{I_{0}^{-}}=\frac{R_{t h}}{\gamma}=\sqrt{\frac{R_{t h}}{G_{t h}+j \omega C_{t h}}}
$$

Where $\gamma=\alpha+j \beta=\sqrt{R_{t h}\left(G_{t h}+j \omega C_{t h}\right)}$ is the complex propagation constant. The series impedance in the model is:

$$
Z_{A}=\frac{R_{t h}}{\gamma} \frac{e^{\gamma L}-1}{e^{\gamma L}+1}=Z_{0} \tanh \frac{\gamma L}{2}
$$

The value for the shunt impedance is:

$$
Z_{B}=\frac{R_{t h}}{\gamma} \frac{2 e^{\gamma L}}{e^{\gamma L}-1}=\frac{Z_{0}}{\sinh \gamma L}
$$
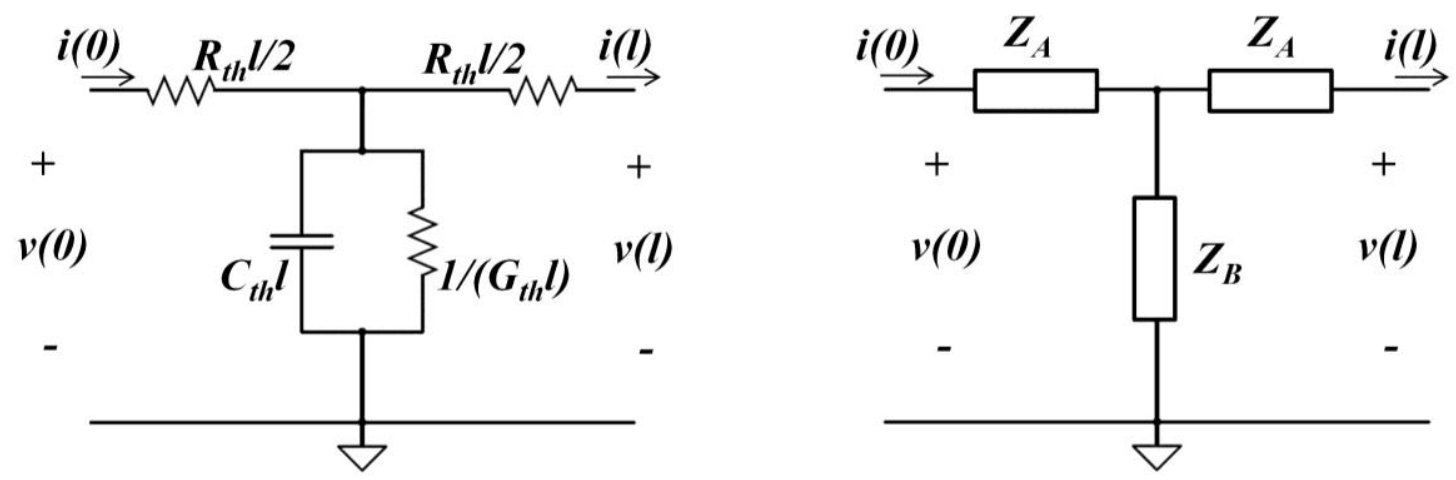

Figure 25: Thermal Transmission Line Model

The voltage waveform representing the temperature on the line in the time domain is:

$$
V(x)=\frac{I(0) R_{t h}}{\gamma} e^{-\gamma x}
$$

It is assumed that the current $I(0)$ is known and expressed in terms of the forward voltage in the line. The voltage may be evaluated at any number of points along the line and its value may be applied to the corresponding nodes in the mechanical domain.

It is possible to obtain the time and position dependent temperature in a beam under $\mathrm{AC}$ excitation with FEA software; however, the FEA solver used in this work requires a transient thermal boundary condition at each frequency of interest, and many time-steps would be required for the model to reach steady state so that the amplitude and phase can be measured. The utility of the distributed parameter 
model is underscored by its rapid convergence, enabling parametric optimization during the design process. The model also provides insight into measurements obtained with scanning thermal microscopy.

\subsubsection{Mechanical Domain}

The mechanical model for a thermal shunt path is the same as a flexure of similar shape. The moment of inertia of the beam varies along its length, since the segments are joined with thin beams. In the mechanical model, the length-weighted average of the thin sections and thick sections can be used to evaluate the value of a single lumped capacitor, because the segments are short. For example, for a cantilever with point-loading in the $z$ direction,

$$
E I_{(e f f, y)}=\frac{E I_{(y, t h i n)} \sum_{\text {segments }} L_{\text {thin }}+E I_{(y, \text { thick })} \sum_{\text {segments }} L_{\text {thick }}}{\sum_{\text {segments }} L_{\text {thin }}+\sum_{\text {segments }} L_{\text {thick }}}
$$

The stiffness and capacitance of such a structure would then be

$$
k=\frac{3 E I_{(e f f, y)}}{L^{3}}, \quad C_{m}=\frac{L^{3}}{3 E I_{(e f f, y)}}
$$

Different values for stiffness may be similarly derived based on geometry and loading conditions.

\subsection{Vertical Electrothermal Actuators}

Vertical electrothermal actuators are used to adjust the tip-sample separation. The static deflection of the vertical actuator is an important quantity, since it is plotted to reveal sample topology. Vertical actuators in this work are based on the thermal bimorph principle; they are a simplified version of the lateral multimorphs discussed in section 3.3.

In many ways, the design requirements of vertical actuators are more demanding than those of lateral actuators. The following are some examples of design constraints.

1. The out-of-plane stiffness of the z-actuator is typically the "weakest link" in the overall out-ofplane stiffness budget, because the actuator must be compliant enough to deflect in this direction. Depending on the type of SPM, $k_{z}$ may vary from $<1 \mathrm{~N} / \mathrm{m}$ to $>50 \mathrm{~N} / \mathrm{m}$.

2. The range of motion of the z-actuator has several impacts on the instrument's performance. A large deflection in the z-direction increases the height of topographical features that can be imaged, and also relaxes the constraint on the resolution of the coarse approach mechanism. A 
large range of motion may be achieved at the cost of stiffness. The range-to-stiffness trade-off can be illustrated for the case of a constant-temperature, tip-loaded bimorph:

$$
z_{\max }=\frac{M L^{2}}{2 E I}, \quad k_{z}=\frac{3 E I}{L^{3}}
$$

In other words, if the length is increased, the range increases as $L^{2}$ while the stiffness decreases as $\frac{1}{L^{3}}$.

3. The bandwidth of the z-actuator is necessarily the highest among all actuators in the SPM. For instance, in order to track topology, the z-actuator must deflect several times to track sample topology within a single line-scan. In addition, for dynamic modes of operation, the actuator must excite a resonant mode in a flexural cantilever, which is usually above the bandwidth of the actuator. The bandwidth may be defined as the location of the thermal pole set by the thermal $\mathrm{RC}$ time constant, which can also be related to the length of the actuator.

$$
f_{-3 d B}=\frac{1}{2 \pi \tau}=\frac{1}{2 \pi R_{t h} C_{t h}}=\frac{\kappa}{2 \rho c_{p} L^{2}}
$$

4. The open-loop position resolution can be defined simply as

$$
\Delta z=\frac{z_{\max }}{2^{n}}
$$

where $z_{\max }$ is the maximum achievable deflection, and $n$ is the number of bits of resolution of the digital-to-analog converter (DAC) that is driving the actuator. This is an oversimplification that does not consider noise contributions to the position resolution of a closed-loop system, as will be shown in section 5.3.1. Nevertheless, the trade-off between position resolution and range is revealed in this expression.

These constraints are intended to illustrate that the z-actuator performance is the result of design tradeoffs that are defined on a case-by-case basis for each type of SPM. Some additional constraints are hard limits that are defined by the process, like the thermal budget and operating voltage. There are, of course, various other constraints that may be defined in terms of material properties and geometry. Some examples are power efficiency, work-per-unit-volume, thermomechanical noise, maximum strain, and damping (squeeze film and thermoelastic). 


\subsubsection{Electrical Domain}

The electrical model of the actuator calculates the amount of joule heating in a resistor that is subject to an applied voltage, and the temperature-dependence of this resistor is captured through feedback from the thermal domain. The input voltage may be divided into a DC component and an AC component. The DC component is applied to track topology, while the AC component is used in dynamic modes. In FM-AFM and AM-AFM, the AC component places the cantilever in resonance to increase the tip-sample force sensitivity. An AC signal may also be used to force a non-resonant oscillation, and subsequently demodulate and filter the output in order to improve noise performance. A schematic of the lumped parameter model in the electrical domain is included in the thermal model in Figure 26 below.

\subsubsection{Thermal Domain}

For quasi-static operation, the thermal model is similar to the lateral actuator model. Following the development of the lumped model from equations (10.49)-(10.61), the values for the components in the circuit are:

$$
\begin{gathered}
C_{t h}=2 A \rho c_{p} \Delta x \\
R_{12}=R_{23}=R_{\text {Cond }}=(\Delta x) / \kappa A \\
R_{\text {conv }}=\frac{T_{a}-T_{2}}{i_{\text {conv }}}=\frac{1}{2 w \Delta x \rho c_{p} h_{\text {conv }}}
\end{gathered}
$$

The schematic of the thermal model with a single lumped capacitor is shown in Figure 26 . If the temperature gradient is sought, the difference between the endpoints of the model may be used to determine the slope of the temperature function. 


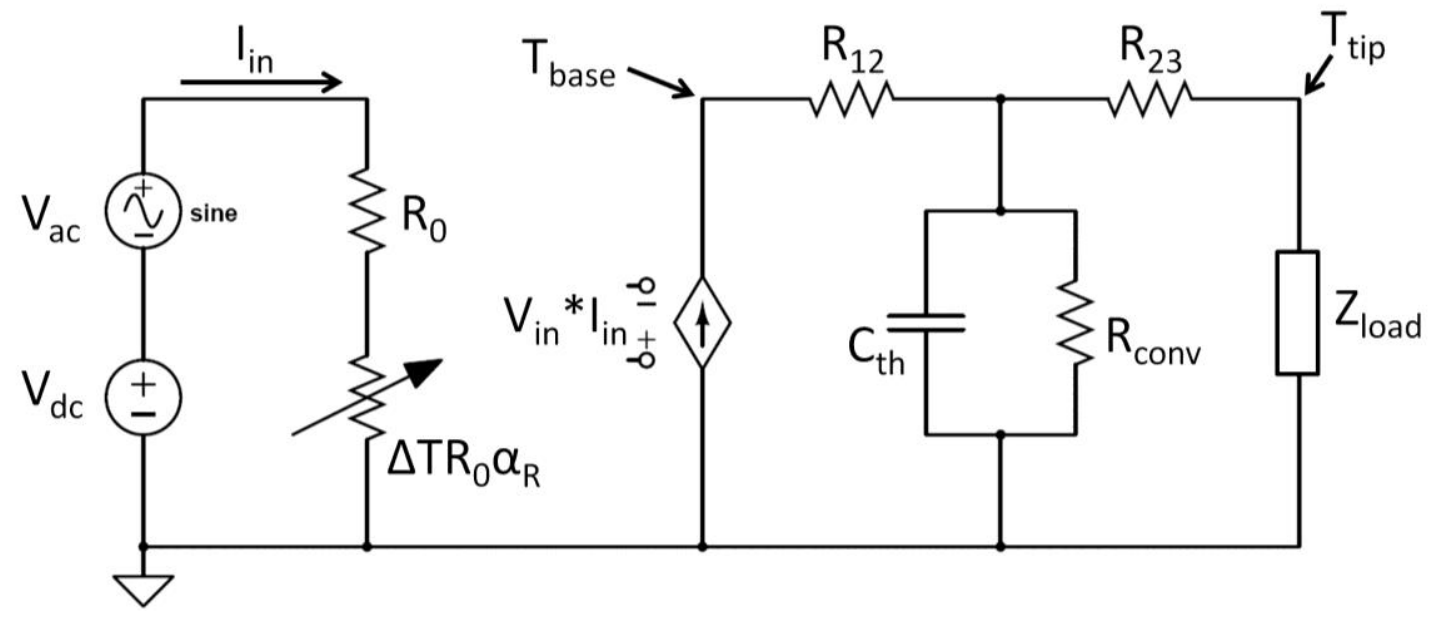

Figure 26: Electro-Thermal Model of a Vertical Actuator.

The introduction of an AC source must be handled in accordance with the power dissipation in the thermal domain. At low frequencies, the contributions of the $A C$ and $D C$ sources may be calculated separately. Because the temperature is proportional to the power dissipated, the instantaneous input voltage is squared to calculate the value of the effort source in the thermal domain. With no DC term, the frequency of the $A C$ temperature is doubled. To avoid this in practise, the minimum value of $V_{d c}$ is chosen to be $1 / 2 \mathrm{~V}_{\mathrm{ac}}$. At high frequencies, well beyond the thermal cutoff, the AC component of the output temperature can be neglected. However, power is still being dissipated in the resistor. In this frequency range, the RMS value of the electrical AC term must appear as a DC term at the thermal output. The temperature of the actuator is proportional to this DC term, so that there is a static displacement at the output. In the intermediate frequency regime, where the AC component is attenuated but still present, the RMS value of the electrical AC signal is split into an AC thermal component and a DC thermal component. The intermediate frequency regime is relevant when a thermal actuator is used to excite mechanical resonance in a cantilever, since the mechanical resonant frequency is usually higher than the thermal cutoff frequency for actuators that are used in this work. Most solvers for lumped element models consider the $A C$ and $D C$ cases in separate simulation environments. The DC component at the output must therefore be calculated for a given operating frequency:

$$
\frac{V_{a c, e l e c}}{\sqrt{2}}=V_{a c, t h}+V_{d c, t h}
$$


Alternatively, a transient simulation may be performed to extract this information, as shown in Figure 27.

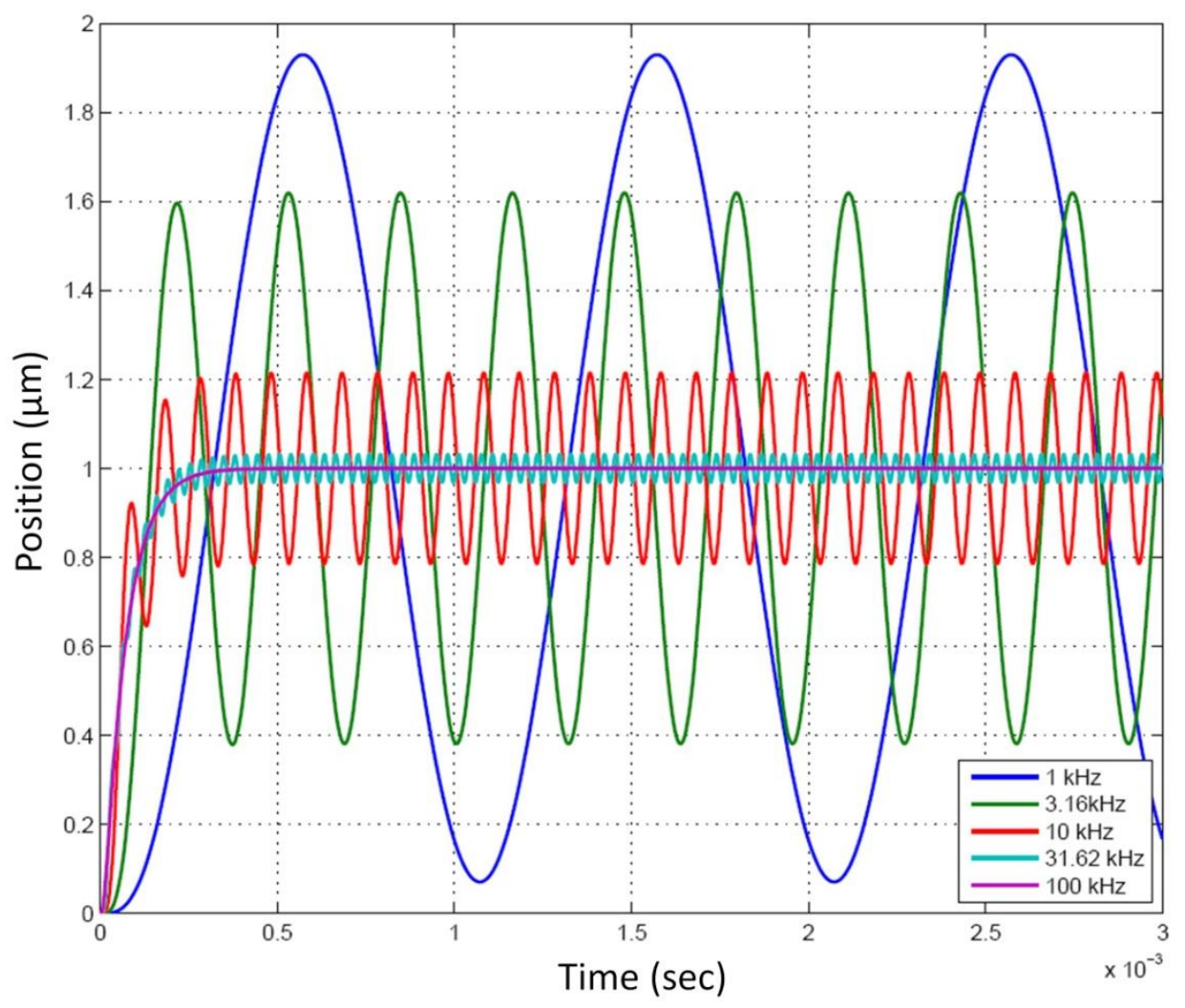

Figure 27: Transient Simulation of a Vertical Actuator at Different Input Frequencies.

Simulation output from transient analysis of thermal transmission line LEM in circuitlab.

\subsubsection{Mechanical Domain}

The temperature distribution from this model is coupled to the mechanical domain via the moment or force source, similar to the lateral actuator of section 3.3. The procedure is simplified for the bimorph case, by virtue of the fact that there are only 2 layers to consider in the cross-section of the beam. First, the location of the neutral axis of the beam is calculated as the modulus-weighted centroid of the beam. Then, the effective bending stiffness of the beam is calculated. Moments from residual stress and thermal stress are then expressed in terms of the material properties and beam geometry. Finally, an expression for the deflection is presented. Details of this derivation process are included in Appendix C.2. As with the lateral actuators, there are three cases that are derived for the mechanical model of the vertical actuator. The most relevant case captures the position dependence of the actuator when it 
is experiencing a temperature gradient. The capacitor that represents the flexural rigidity of the beam is used when tip angle is desired:

$$
C_{\theta}=\frac{1}{k_{\theta}}=\frac{L}{(E I)_{e f f, y}}
$$

The voltage source representing the thermal moment is:

$$
V_{t h, \theta}=\frac{L^{2} \Delta T}{2}\left[S_{M_{-} y, t h}+\frac{m_{t h} L}{3 \Delta T}\right]
$$

If the model seeks to determine the tip deflection, energy methods are used to capture the strain energy in the beam. The stiffness of the beam in this case is represented by a capacitor with a value of:

$$
C_{z}=\frac{1}{k_{z}}=\frac{L^{3}}{4(E I)_{e f f, y}}\left[\frac{1}{3}+\frac{L m_{t h}+2}{\left(L^{2} m_{t h}^{2}+3 L m_{t h}+3\right)}\right]
$$

And the voltage is:

$$
V_{t h, z}=\frac{q}{C_{z}}=2 S_{M_{-} y, t h} \frac{L^{2} m_{t h}^{2}+3 L m_{t h}+3}{L\left(L m_{t h}+3\right)} \Delta T
$$

In the model shown in Figure 28 below, the displacement at the tip of the actuator is represented by the charge $q$ on the capacitor $C_{z}$. At frequencies above the thermal RC cutoff, the temperature distribution along the length of the actuator becomes important. In order to couple temperature values from multiple points in the thermal model to corresponding points in the mechanical model, one must consider both the tip angle and displacement, since the initial angle of a given segment contributes to the deflection at the end of the segment. The overall deflection is thus weighted by the product of the angle and distance to the tip, as shown in the complete model. 


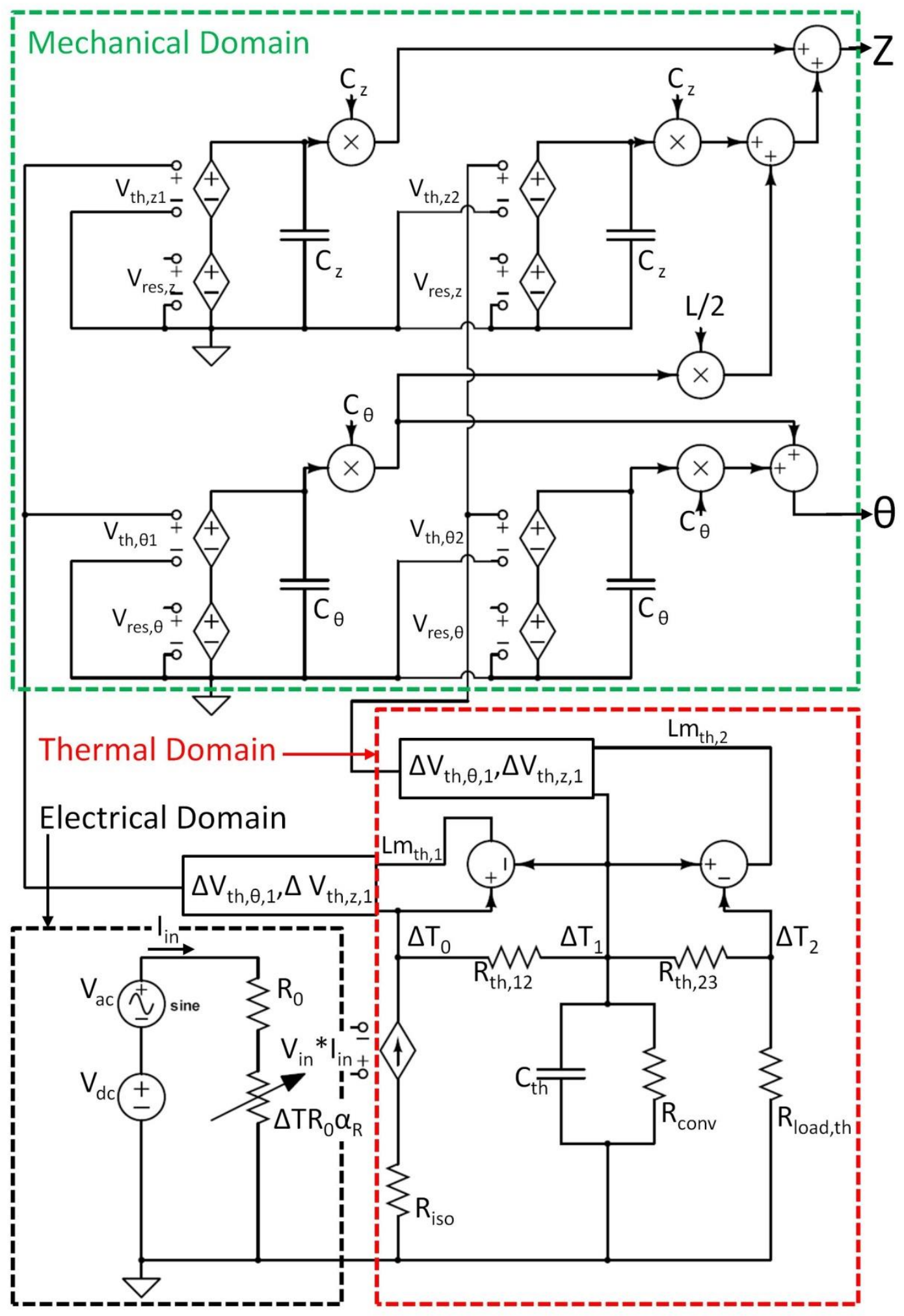

Figure 28: Electro-Thermo-Mechanical Model of a Vertical Actuator 


\subsection{Temperature Sensors}

The influence of local temperatures on the operation of single-chip SPM's has been described in several of the component models presented so far. The model of a self-heated resistor presented in section 3.2 illustrates the coupling between the electrical and thermal domains, and methods to linearize the output temperature and mitigate thermal runaway issues. In addition, it was observed that for bimorph geometries in which the resistor experiences both strain and heat, the piezoresistance effect may dominate over the TCR.

Temperature sensors are used to measure heat transfer at the tip-sample region in Scanning Thermal Microscopy. The sensors may use either the TCR of the polysilicon material, or the Seebeck effect at the polysilicon-to-metal thermocouple. The electrical domain representation of the resistive temperature sensor is similar to that of a joule-heating resistor. The thermocouple is modeled as a temperaturedependant voltage source in the electrical domain. The heat capacity of the temperature sensor is important when harmonic temperature variations occur at the tip-sample interface. Thus the thermal domain representations must capture dynamic effects.

\subsubsection{Resistive Temperature Sensors}

The design considerations for resistive temperature sensors are different from those for heaters. The model of a resistive temperature sensor is the similar to that of a self-heated resistor placed in a bridge circuit to detect the change in resistance. A self-heating resistor should be in good thermal contact with an actuator in order to improve efficiency, whereas a resistive temperature sensor should be thermally isolated to improve sensitivity to an input temperature. In the model, the input temperature to be measured is that of the sample, and the heat flow occurs in the tip-sample region. Ideally, heat flow from the rest of the instrument should be minimized, which is the motivation behind the thermal isolation chain. Another difference is that the heater resistor circuit should be designed to prevent thermal runaway at high temperatures, whereas the resistive sensor circuit should be designed to improve resolution at low temperatures. In the model of the resistive sensor, a current source is used in conjunction with a negative TCR resistor. The differential equation governing temperature in the current driven case is

$$
\frac{d T_{R}}{d t}=-\left(\frac{1-\alpha_{R} R_{0} R_{t h} I^{2}}{R_{t h} C_{t h}}\right) T_{R}+\frac{I^{2} R_{0}}{C_{t h}}
$$


which is different from the voltage driven case in equation (3.3). In this case, the thermal time constant is:

$$
\tau_{I}=\frac{R_{t h} C_{t h}}{\left(1-\alpha_{R} R_{0} R_{t h} I^{2}\right)}
$$

The steady state temperature can now be expressed as:

$$
T_{S S}=\frac{R_{0} R_{t h} I^{2}}{1-\alpha_{R} R_{0} R_{t h} I^{2}}
$$

which may be recognized as a system with negative feedback for negative $\alpha_{R}$. Since $\alpha_{R}$ is small in the resistive sensors used here, one may choose to drive the sensor with a voltage source in order to benefit from positive feedback. However, the voltage setting should still be limited to prevent thermal runaway.

\subsubsection{Thermocouples}

The CMOS-MEMS process may also be used to implement temperature sensors based on the thermoelectric effect, using a pair of junctions between materials with different Seebeck coefficients. When the junctions are at different temperatures, a potential difference is generated. SEM images of a design with a resistive temperature sensor and one with a thermocouple are shown in Figure 29 for comparison. The routing for the thermocouple must use the same materials that are at the junction, otherwise the thermovoltages would cancel. Only one thermal isolation chain is required, since the polysilicon is beneath the metal layers used in the chain.
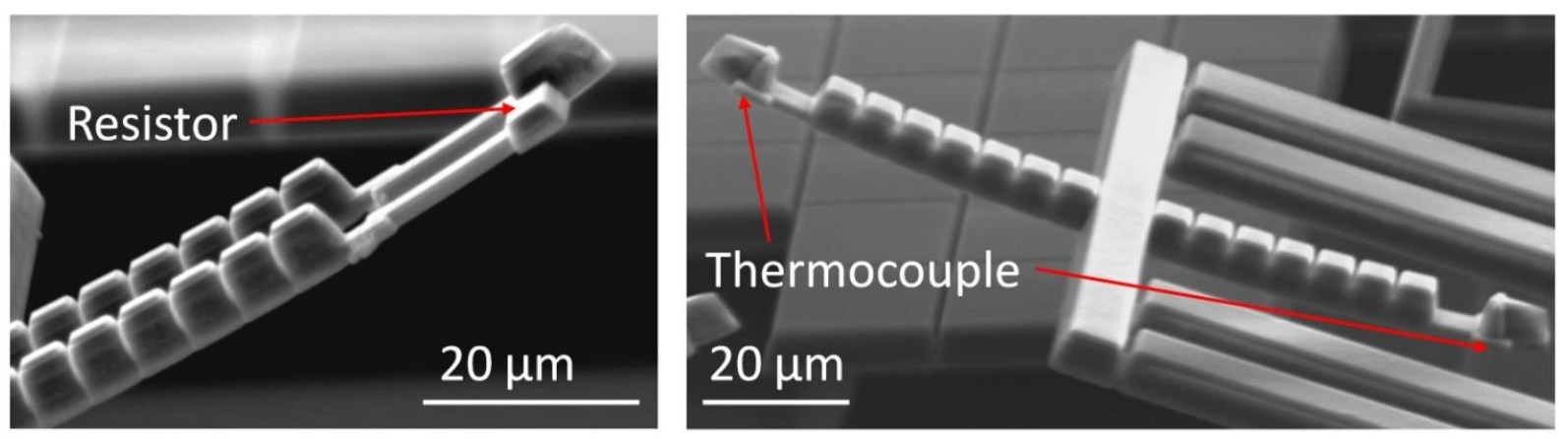

Figure 29: Resistive Temperature Sensor (Left) and Thermocouple (Right) 


\subsubsection{Electrical Domain}

The voltage generated by the thermocouple arises from the coupled flow of electrical and heat currents, which is described by irreversible thermodynamics [149]. In a CMOS process, there are several materials with different Seebeck coefficients $\alpha_{s}$ that may be coupled to form junctions in the design process. Thermocouples of high resistivity polysilicon and Aluminum are used in this work. The voltage generated by the thermocouple is:

$$
V_{T C}=\int_{T_{C}}^{T_{h}}\left(\alpha_{\text {poly }}-\alpha_{A l}\right) d T
$$

The value of the voltage source depends on the difference in temperature between the two junctions on either end of the thermocouple. The temperature at the tip is influenced by tip-sample heat conduction, whereas the temperature at the base is influenced by heat from the remainder of the instrument. In practise, a self-heated resistor may be placed at the base of the thermal isolation chains, in order to maintain a fixed reference temperature above the sample temperature. In this case, the tip is the "cold" junction. This heater may be operated in a feedback mode, in order to compensate for temperature variations in the device. Alternatively, the heater may be used to provide an AC heat current which modulates the "hot" junction temperature, such that lock-in techniques may be used to extract the temperature difference. It is important to note that in this case, AC modulation does not offer immunity to the temperature variations in the remainder of the device. This is because the temperature variations disturb value of the sensor's output intrinsically, as opposed to adding random noise. Thus, upon demodulation, these temperature variations will reappear directly in the signal of interest. Therefore, modulation of the base temperature has the same effect as modulating the drive current of a resistive temperature sensor: it limits the bandwidth of the Johnson and $1 / f$ noise contributions to the output signal. Sensitivity is further improved by the addition of thermal isolation between junctions at either end of a thermocouple. The electrical domain model of the thermocouple is simply a temperature dependent voltage source.

\subsubsection{Thermal Domain}

The temperature at either end of the thermocouple is required in order to calculate the voltage that is generated in the electrical domain. A heater resistor is placed near the base of the device in order to modulate temperature to enable lock-in measurement techniques. The thermal path from the base to the tip is modeled using a thermal transmission line, because the phase and amplitude difference 
between these points contributes directly to the output signal. It is important to consider the frequency dependence of the thermal transmission line when selecting an operating frequency for SThM measurements. Ideally, the thermal impedance of the transmission line should be matched to the tipsample impedance to maximize the sensitivity of the measurement. In ambient conditions, with a relatively large tip radius ( $100 \mathrm{~nm})$, the CMOS-MEMS process may achieve good impedance matching; however, for very sharp tips in vacuum operation, process modifications may be needed to achieve this goal. The impedance of the thermocouple may therefore be a limiting factor in the temperature and spatial resolution of an SThM instrument.

\subsubsection{Mechanical Domain}

The position of the tip of the thermocouple is an important quantity, since it influences the amount of heat transfer to the sample. The position of the base of the thermal isolation chain is set by the vertical actuator to which it is connected. A small additional deflection occurs due to the bimorph-like behaviour of the thermal isolation chain. The temperature distribution from the thermal domain may be used to calculate the thermal moment in the thin (metal_1) segments of the chain. The bending stiffness of the chain is also dominated by the thin connecting segments. In the model below, the rotation of the tip is obtained simply by considering the only the lengths of the metal_1 segments in the capacitor. To calculate the vertical deflection of the tip, one would include the angular projection over the length of the thick segments. It is assumed that the small angular deflection of the tip directly modulates the tip-sample gap, as indicated by the variable load impedance in the thermal domain.

A complete model of the thermocouple is included below in Figure 30. 


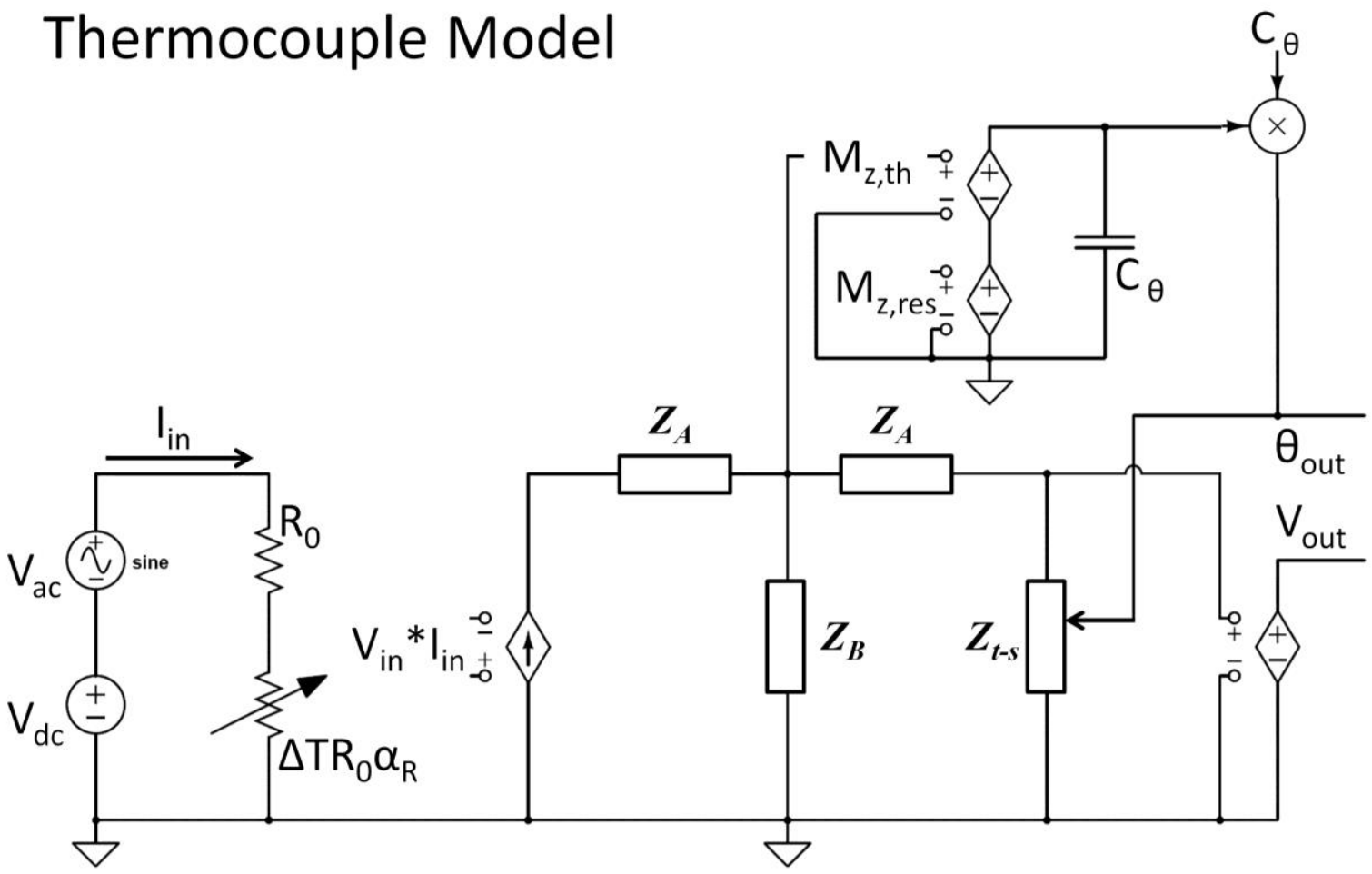

Figure 30: Thermocouple Model 


\section{Chapter 4. Quality Factor Enhancement in Resonant Cantilevers with Integrated Piezoresistors}

The components that have been modeled in Chapter 3 may be combined into scanners that can be used to position a sharp tip over sample, which is a fundamental requirement in the operation of SPM's. The measurement of the tip-sample interaction is based on a sensor that can preferentially detect quantities that are of interest. In AFM, the tip-sample interaction force is detected by measuring the deflection of a cantilever. If the cantilever is placed in resonance, its sensitivity to an applied force is enhanced, as will be shown in this chapter.

In a single-chip AFM, the piezoresistive effect is used to detect the deflection of a cantilever. A polysilicon resistor with modest piezoresistivity is integrated into a composite beam of Aluminum and Silicon Dioxide. Because the beam is made of two materials with dissimilar thermal expansion coefficients, it deflects in response to a temperature rise, producing unwanted strain in the piezoresistor that detracts from tip-sample interaction measurements. The dynamics of the beam are determined by its shape and the material properties of its constituents. In this work, the beam itself is modeled first, since the thin polysilicon layer has minimal influence on its mass, stiffness, and resonant frequency. Once the dynamics of the beam are uncovered, the piezoresistor is modeled. The coupling between the two occurs in the thermal domain through self-heating, and in the mechanical domain through strain from resonance and from bimorph actuation. Once the piezoresistor is modeled, it will be shown that under certain circumstances, the presence of a piezoresistor may have a significant impact on the dynamics of the cantilever.

\subsection{Flexural Resonant Cantilevers}

Flexural resonant cantilevers are used to improve the sensitivity of the piezoresistors to tip-sample interaction forces. The sensitivity of the force measurement is improved by the Q-factor of the resonator, up to a factor of $Q^{2}$ in the case where the displacement sensor noise dominates over the cantilever's thermomechanical noise [156]. This section focuses on the electrical, thermal and mechanical models of the flexural resonators themselves, while section 4.2 discusses the piezoresistors that are embedded inside them.

There are two approaches to resonant cantilevers that are explored in this work. The self-actuated resonant cantilever (SARC) consists of a bimorph beam with an integrated heater and piezoresistor. In 
essence, the SARC is a vertical electrothermal actuator that uses the same polysilicon resistor for piezoresistive detection and Joule heating for vertical actuation to track topology. The temperature distribution in the vertical actuator may be modeled using the finite difference method, with a dependant resistance that is modulated by the TCR and the coefficient of piezoresistivity. This is problematic because heat from thermal actuation couples directly to the strain sensors, and obscures the signal from tip-sample force interaction. The term "feedthrough" is often used to describe similar coupling problems resulting from capacitive coupling in the electrical domain, and in this case the phenomenon is more accurately described as "thermal feedthrough." Separating the heaters from the sensor and placing them at opposite ends of the cantilever may mitigate the thermal feedthrough issue, at the expense of either the efficiency of the actuator, or the sensitivity of the piezoresistor. If the piezoresistor is placed at the base and the heater at the tip, the efficiency of the actuator is reduced. Conversely, with the piezoresistor at the tip and the heater at the base, the force sensitivity is compromised. Some insight into the SARC design pattern will be provided in section 4.2 .5 , which discusses the dynamic model of piezoresistive strain sensors.

Some recent research effort has focused on electrothermally actuated, piezoresistively detected micromechanical resonators [157]. These "I2BAR" devices with resonant frequencies up to $176 \mathrm{MHz}$ use the same resistors for heating and piezoresistive sensing, which implies that they experience both thermal and electrical feedthrough. At such high frequencies, electrical feedthrough is more significant than thermal feedthrough because the thermal time constants are so short that the AC temperature amplitude is low. In this work, the frequencies are low enough that thermal feedthrough is significant, but electrical feedthrough may be eliminated by the use of separate resistors for heating and sensing.

Based on the preceding discussion of the drawbacks of the SARC pattern, conventional wisdom would guide the designer towards a design in which the heater and sensor are separate. Nevertheless, it was desirable to investigate the effects of thermo-mechanical coupling within a self-heated, strain-sensing resistor. As it turns out, the Q-factor of the SARC may be enhanced by internal feedback mechanisms that result from the interplay of quantities in the electrical, thermal, and mechanical domains. In addition, the use of such a design pattern in a Wheatstone bridge configuration provides an excellent match at a frequency near the mechanical resonance. For a single-chip SPM to utilize this enhancement, a separate vertical actuator would be required for tracking topology, so the design is referred to as a hybrid mode of SARC operation. This phenomenon will be discussed in section 4.2 below. 
The approach discussed in this section is the externally actuated resonant cantilever (EARC), which uses a vertical bimorph actuator or a rotating actuator to excite a flexural mode within a beam that has an integrated piezoresistor, as shown in Figure 31. The external vertical actuator may be thought of as a "shaker," similar to the piezoelectric actuators used in conventional AFM's. The actuator is designed with multiple beams to increase its stiffness, resulting in a higher resonant frequency than the flexure. A higher resonant frequency also improves the loaded $Q$ of the flexural resonator by undercoupling the resonators. To further separate the resonant frequencies of these components, a proof mass may be added to the tip of the flexural resonator. The EARC design has several advantages over the SARC design, which were observed over the course of this work:

1) The EARC has improved thermal immunity, since the piezoresistor may be located further from the heat source. In addition, at high frequencies, the thermal wave from the z-actuator constricts, resulting in further suppression of heat coupling. By contrast, the thermal bending moment that is responsible for exciting the flexural resonance is collocated with the piezoresistor in the SARC.

2) The EARC is more robust to tip-crashes, since the stiffness of the vertical actuators can be high enough to overcome pull-in forces without compromising the force sensitivity of the sensor that is in a separate, more compliant cantilever. By contrast, in the SARC, if the vertical actuator is stiffened, the force sensitivity of the integrated strain sensor is reduced.

3) The EARC design pattern supports higher resonant frequencies. As the resonant frequency of the flexural cantilever is increased (by reducing its length or the tip mass), the thermal envelope in the stiff vertical actuator is compressed further, improving the thermal immunity of the strain sensor. In the SARC design, the strain sensor shrinks along with the thermal envelope, and so it is still co-located with the high AC temperature region.

It should be noted that the EARC may be operated in a hybrid mode in which the piezoresistive sensors are used for resonant actuation and the external vertical actuator is used to track topology. This mode benefits from the Q-factor enhancement of the SARC with reduced thermal coupling from the external actuator that tracks topology.

\subsubsection{Electrical Domain}

The electrical model of the EARC is simply a conductive path that does not contribute to its thermal or mechanical behaviour. This conductor is often the only path that may be used to route signals to the tip 
of the instrument. Since the signals to the tip are often low current signals that are susceptible to noise, a capacitor may be included to capture the coupling between the underlying piezoresistor and the EARC conductor. An AC current arises in the piezoresistor at resonance because the change in resistance modulates the DC bias that is applied to it. The model that considers these coupling effects is similar to the electrical routing model in a flexure that was shown in Figure 15. In the EARC model, the cross section of the beam only consists of 1 metal layer and the piezoresistor, and the metal layer may be used to route a signal to the tip. This signal may be an AC signal as required by Scanning Capacitance Microscopy (SCM), Scanning Thermal Microscopy (SThM), Scanning Gate Microscopy (SGM) or Kelvin Probe Force Microscopy (KPFM); or it may be a DC signal, as required by Scanning Spreading Resistance Microscopy (SSRM), Conductive AFM, or Scanning Voltage Microscopy (SVM). In these cases, the parasitic capacitance couples the tip signal to the piezoresistor signal, contributing parasitic AC feedthrough in both directions. There are two solutions to the feedthrough problem. The first is to rely on frequency domain techniques to separate the signals, since the resonant frequency of the AFM may be different from the signal to the tip. This method does not always work, since it may be desirable to use lock-in detection techniques based on the modulation of the tip signal by the cantilevers resonance. Another solution is to route the tip signal in a separate flexure that is placed in parallel with the EARC. This solution increases the effective stiffness of the system, thereby reducing the strain captured by the piezoresistor. Examples of both approaches are shown in Figure 31.
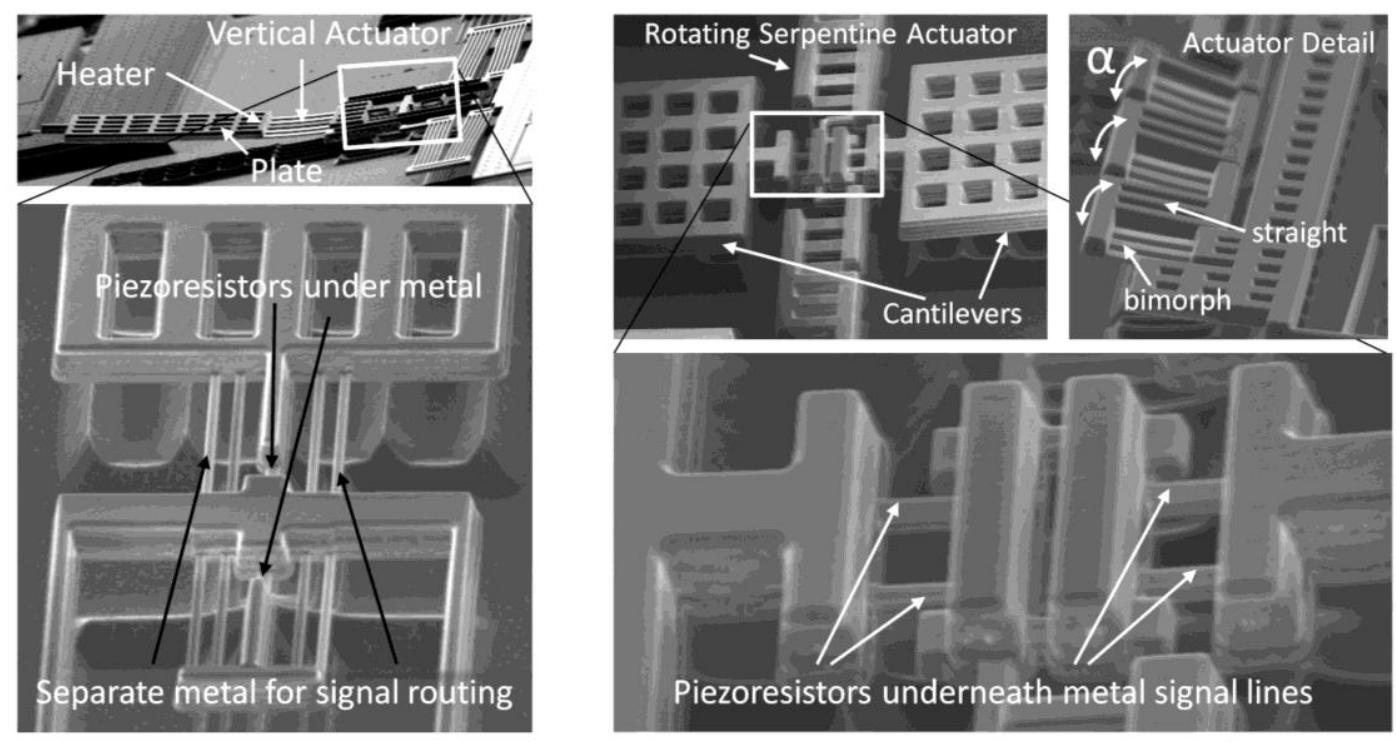

Figure 31: Externally Actuated Resonant Cantilevers 


\subsubsection{Thermal Domain}

In the thermal domain, the EARC model and the embedded piezoresistor model are inexorably tied together, because it is assumed that the temperature remains constant over the cross-section of the beam. This assumption is verified by FEA. In the cross section of Figure 22 the heat flux through the members in the beam was shown to vary as a function of their thermal conductivity. In the same analysis, the temperature range over the cross section of the beam was found to remain within $\sim 5 \mathrm{~K}$, while there is a $150 \mathrm{~K}$ temperature difference over the length of the beam.

The temperature in the EARC is influenced by heat entering or leaving from either end, and by the heat generated in the piezoresistor. If a thermal gradient exists along the beam length, the model is split into segments with dimensions that are based on the location of the piezoresistor within the beam. One may choose to represent the component with a transmission line model or a lumped parameter model. Because the heater is located at a distance from the piezoresistor in a well-designed EARC, the temperature variations at the location of the piezoresistor have a small amplitude and a different phase compared to the signal from resonance. Under these conditions, the lumped parameter model suffices. The distal end of the EARC may be extended by a plate, as shown in Figure 31. The convection from this plate is captured by a load resistor placed at the distal end of the model. When the tip approaches a sample, heat is conducted through the gap between the plate and the sample. This conductive transfer is modeled with a separate resistor with a value that depends on the geometry of the air gap and the material properties of the sample. Assuming that the thermal impedance of the substrate-air gap is large compared to the thermal impedance in the substrate, the value of the resistor that captures conductive heat transfer is:

$$
R_{\text {gap }}=\frac{\frac{l_{p l t}}{2} \sin \theta+d_{t-s}}{k_{\text {air }} w_{p l t} l_{p l t}}
$$

where $l_{p l t}$ and $w_{p l t}$ are the length and width of the plate, $\theta$ is the angle between the plate and sample, $d_{t-s}$ is the tip-sample distance, and $k_{\text {air }}$ is the thermal conductivity of air. Here, it has been assumed that the Raleigh number (from equation (10.28)) is small, so that only conductive effects are considered in the plate-sample gap. The thermal model of the EARC is shown in Figure 32. 


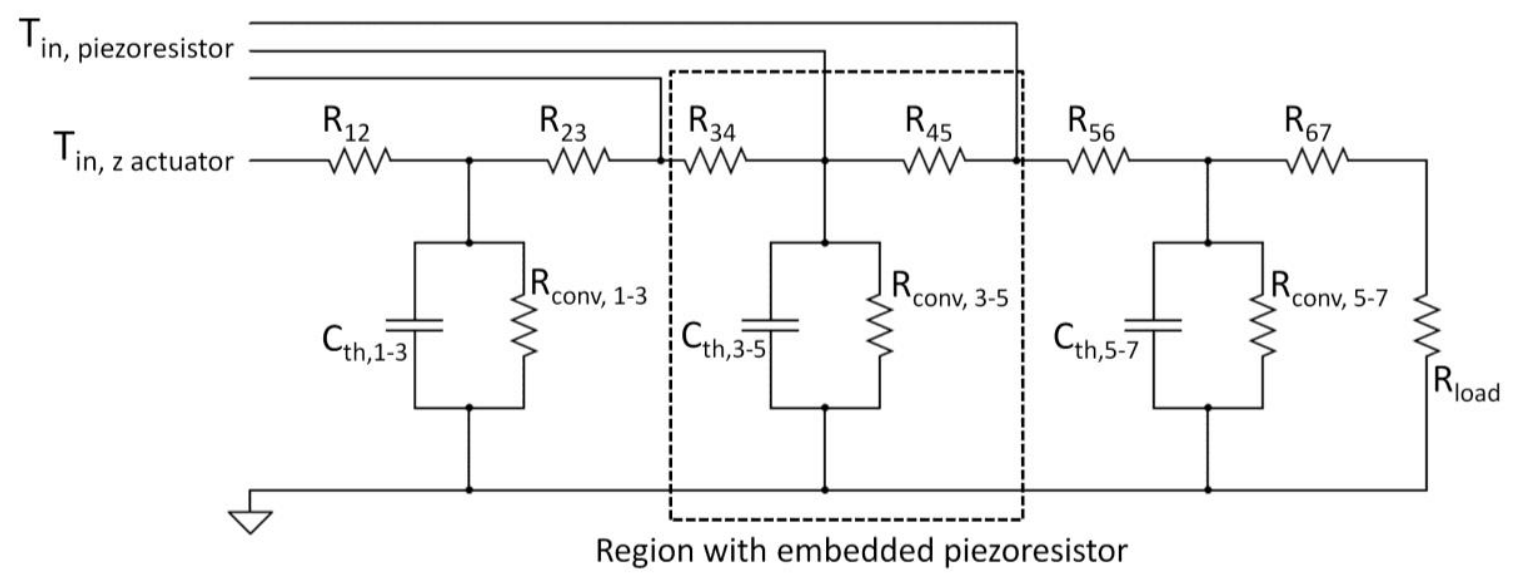

Figure 32: Thermal Model of Externally Actuated Resonant Cantilever

\subsubsection{Mechanical Domain - Quasi-Static Model}

The cross section of the EARC is similar to that of a vertical electrothermal bimorph actuator, except in the region where the piezoresistor is embedded. In that region, the multimorph formulation from equation (10.67) may be used to calculate the effective bending stiffness, with the coordinate system rotated to match out-of-plane bending.

$$
(E I)_{e f f, y}=\sum_{m e m b e r s} \int_{y_{i}}^{y_{i}+\Delta y_{i}} \int_{z_{i}}^{z_{i}+\Delta z_{i}} E_{i}(z) z^{2} d z d y
$$

where the members include the oxide, polysilicon, and metal layers. In the regions where there is no polysilicon, equation (10.133) may be used to calculate the effective bending stiffness. The beam experiences a moment from residual stress, a moment from thermal stress, and a moment from tipsample interaction. The moment from tip-sample interaction is:

$$
M_{y, F_{t-s}}(x)=F_{t-s}\left(L_{c a n t}-x\right)
$$

The other moments can be calculated using equations (10.134)-(10.135), where it is assumed that the thin, short polysilicon sensor with a thermal expansion coefficient close to that of $\mathrm{SiO}_{2}$ (when compared to that of Aluminum) does not contribute to deflection. The self-heating of the sensor does contribute to the thermal moment in its section of the beam, as indicated by the coupling to the thermal domain. Since the temperatures from the thermal model of the piezoresistor consider self-heating, the calculation of the thermal moment is straightforward. The values of the voltage sources and capacitors corresponding to the moments and stiffness of the beams are calculated using (10.144)-(10.145). 
Values for the capacitors and voltage sources in the tip-referred case may be calculated using (10.147)(10.148). A scaling factor must be used for the moment from tip-sample interaction force because the capacitor used in the model does not represent the cantilever spring constant from a tip load. The scaling factor accounts for the difference between tip-loading and moment-loading from thermal actuation, as discussed in section 3.3.3. Like the thermal model, the mechanical model is subdivided into sections based on the location of the strain sensor. The moments and deflections are therefore calculated at various points along the beam. The quasi-static model of the EARC in the mechanical domain is shown below in Figure 33. This model only considers 2 segments, corresponding to the case where the piezoresistor is located at the base of the beam.

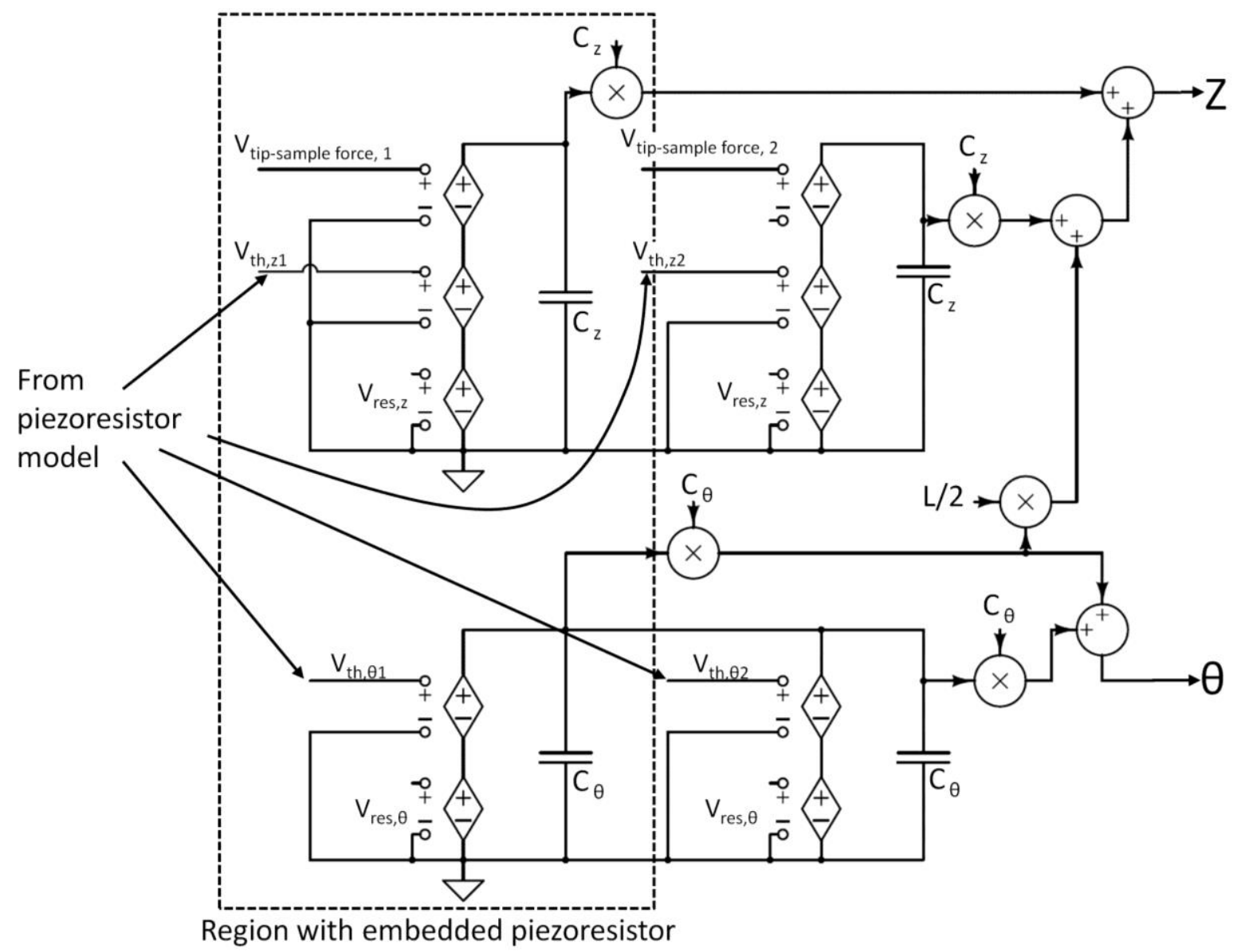

Figure 33: Thermal Domain Model of Externally Actuated Resonant Cantilever

\subsubsection{Mechanical Domain - Dynamic Model}

When the beam is excited at an AC signal close to its resonant frequency, its mechanical dynamics come into play. The EARC is excited at its base by a vertical bimorph actuator that provides a linear 
displacement with a small rotation. In addition, the beam's DC temperature contributes a constant thermal moment that pre-stresses it. FEA shows that for a beam at room temperature with the dimensions considered here, the natural frequency is $34981 \mathrm{~Hz}$. The frequency is reduced to $34216 \mathrm{~Hz}$ when the temperature is increased to $400 \mathrm{~K}$, presumably due to the temperature coefficient of elasticity, combined with the flattened shape of the beam. Although these factors contribute to a shift in the resonant frequency, the beam is assumed to be in free-vibration to calculate the natural frequency.

The lumped element model of the dynamic EARC consists of a resistor to represent damping, a capacitor to represent compliance, and an inductor to represent the effective mass. To obtain values for these quantities, the natural frequency of the mode of interest is first obtained. The time-dependent EulerBernoulli beam equation may be solved using the method of separation of variables with the following boundary conditions:

$$
\begin{aligned}
& E I\left(\frac{\partial^{4} w}{\partial x^{4}}\right)=-\mu \frac{\partial^{2} w}{\partial t^{2}} \\
& \text { at } x=0: \quad w=0, \quad \frac{d w}{d x}=0 \\
& \text { at } x=L: \quad \frac{d^{2} w}{d x^{2}}=0, \quad \frac{d^{3} w}{d x^{3}}=0
\end{aligned}
$$

where $\mu$ is the mass per unit length of the beam. Separating $w(x, t)$ into position-dependent and timedependent functions:

$$
w(x, t)=X(x) T(t)
$$

The beam equation can now be separated into two ordinary differential equations:

$$
\frac{\partial^{2} T(t)}{\partial t^{2}}+\omega^{2} T(t)=0, \quad \frac{\partial^{4} X(x)}{\partial x^{4}}-a^{4} X(x)=0
$$

where the dimensionless wavenumber $a$ is related to the angular frequency $\omega$ by:

$$
a^{4}=\frac{\mu \omega^{2}}{E I}
$$

The solution to the position dependent function is: 


$$
X(x)=C_{1} \sin (a x)+C_{2} \cos (a x)+C_{3} \sinh (a x)+C_{4} \cosh (a x)
$$

where $C_{i}$ constant coefficients. The solution to the time dependent function is:

$$
T(t)=d_{1} \sin (\omega t)+d_{2} \cos (\omega t)
$$

where $d_{i}$ are constant coefficients. Applying the boundary conditions results in the following expression for the mode shape:

$$
w_{n}(x)=\frac{A}{2}\left\{\cosh \left(\frac{\lambda_{n} x}{L_{\text {cant }}}\right)-\cos \left(\frac{\lambda_{n} x}{L_{\text {cant }}}\right)+C_{n}\left[\sin \left(\frac{\lambda_{n} x}{L_{\text {cant }}}\right) \sinh \left(\frac{\lambda_{n} x}{L_{\text {cant }}}\right)\right]\right\}
$$

where $\lambda_{n}$ and $C_{n}$ are integration constants. For the first mode, $n=1, \lambda_{1}=1.875$ and $C_{n}=0.734$.

The natural frequency (or the first mode) of the beam is:

$$
\omega_{1}=\left(\frac{\lambda_{1}}{L}\right)^{2} \sqrt{\frac{E I}{\mu}}
$$

The dynamic model represents discrete modes that are obtained from the time-dependent solution of the Euler-Bernoulli beam equation. Several modes can be connected in parallel if one wishes to capture higher modes in the model. The value of the capacitor is:

$$
k_{e f f, 1}=\frac{3 E I}{L^{3}} \quad C=\frac{L^{3}}{3 E I}
$$

The effective mass can be calculated from:

$$
\omega_{1}=\sqrt{\frac{k_{e f f, 1}}{M_{e f f, 1}}} \quad \Rightarrow \quad M_{e f f, 1}=\frac{3 \mu L}{\lambda_{1}^{4}}
$$

And the resistance $R$ that represents damping in the system may be fitted from experimental results. The unloaded Q-factor of the EARC may be extracted from measurements using the concepts from [158].

\subsection{Piezoresistive strain sensors}

Piezoresistivity is a material property whereby the bulk resistivity of a material changes under applied strain. Piezoresistive strain sensors are used in AFM and related SPM modes that must detect small tip- 
sample forces. The piezoresistance of polysilicon has been widely investigated [159],[160],[161][162]. The design of piezoresistive-based MEMS sensors has been analyzed from a system perspective, to quantify the achievable dynamic range while considering noise contributions from various components in the system [163]. The optimization of single-crystal-silicon piezoresistive u-shaped cantilevers for AFM application has also been reported in [164]. The sensitivity was optimized by varying the length, width, and thickness of the cantilever, as well as the dopant depth, dopant concentration, and surface treatment and anneal process parameters. Within a CMOS-MEMS process, the foundry controls the thickness and vertical position of the polysilicon layer, as well as all the process parameters. The polysilicon material is therefore not optimized for piezoresistivity, and the optimization problem is reduced to the sizing (width and length) and placement of the sensor within a flexure.

The piezoresistors are fabricated using the high-value polysilicon material, since this material usually has the highest gauge factor among the released layers in the CMOS-MEMS process [68], [165]. When the grain orientation is random, the material can be considered isotropic. In addition, since the piezoresistive layer is thin, it is assumed that it does not contribute to the effective stiffness $(E I)_{e f f}$ of the beam. The vertical placement of the sensor near the bottom surface of a flexure means that when the beam is bending, the sensor experiences longitudinal strain. These considerations imply that the change in resistance is proportional to the local bending moment.

Piezoresistivity is defined as:

$$
\rho_{\sigma}=\frac{\left(\frac{\partial \rho}{\rho}\right)}{\epsilon}
$$

where $\partial \rho$ represents the change in resistivity, $\rho$ is the original resistivity of the material, and $\epsilon$ is the applied (uniaxial) strain. In addition to piezoresistive effects, the resistor value changes due to its deformed geometry under strain. For a material with no piezoresistivity, the change in resistance is:

$$
\frac{\Delta R}{R}=\epsilon(1+2 v)
$$

The direction of current flow may be in the same direction as the applied strain (longitudinal), or in the perpendicular direction (transverse). The Gauge Factor (GF) of a material is defined as the fractional change in resistance, $R$, of a material per unit strain, $\epsilon$. For the longitudinal case, GF is given by: 


$$
G F_{l}=\frac{\Delta R}{R \epsilon}=2 v+\rho_{\sigma}
$$

Because the piezoresistive layer is thin and embedded within the beam material, only the last term in equation (4.16) will be used to calculate the resistance change due to strain in a flexure. Another way to express the fractional resistance change is to use the piezoresistive coefficients $\pi$, which are related to stress:

$$
\frac{\Delta R}{R}=\pi_{l} \sigma_{l}+\pi_{t} \sigma_{t}
$$

It is desirable to exclusively measure tip-sample interaction forces with these sensors; however, several other factors contribute to the sensor's output signal [150]. First of all, the magnitude of the TCR in the material is so high that it may obscure the signal from strain. In fact, for a given tip deflection, the signal from thermal effects may be $20 x$ greater than the signal from strain effects. Exacerbating this problem is the fact that the piezoresistive coefficient itself is sensitive to temperature - the sensitivity of the piezoresistor is reduced as the temperature is increased. This results in a modified expression for the piezoresistivity:

$$
\rho_{\sigma}=\rho_{\sigma_{o}}\left(1+\alpha_{\rho_{\sigma}}\right)
$$

In addition to the parasitic thermal effects, the sensor is embedded in a bimorph beam. Even if the bimorph is not intended to operate as an actuator, it will deflect in response to local temperature variations, and the resulting strain is captured by the piezoresistor. A third source of parasitic coupling is capacitive coupling from signals routed over the sensor. If the thermal actuator signal is routed over the piezoresistor, the coupled signal is at the same frequency as the desired measurement. Finally, if the sensors share a ground wire with other components of the system, the finite resistance of the wire causes coupling of the shared signals into the sensor output. This phenomenon is known as "ground bounce." The model of the piezoresistor must therefore account for electrical, thermal and mechanical effects. All of these sources contribute to the sensor output even when there is no tip-sample interaction. It is therefore important to include these parasitic sources in the model, and to use the model to guide the design process of the instrument with the objective of reducing these effects.

A bias voltage is applied to a Wheatstone bridge circuit that contains the piezoresistor. The power in the piezoresistor causes "self-heating," which is source of the thermal feedback. In addition, since the piezoresistor must be placed inside a bimorph due to process constraints, its temperature causes 
parasitic strain from deformation, which is the source of the mechanical feedback. Since the length of the piezoresistor is short, distributed thermal effects are negligible. In the mechanical domain, a capacitor is used to represent the compliance of the cantilever. The strain that is experienced by the piezoresistor can be captured by considering the charge on this capacitor. The dynamic behaviour of the beam containing the piezoresistor is important when it experiences flexural resonance. In this case, a mass-spring-damper model may be used. The strain in the piezoresistor in the resonant case must be calculated based on the resonant mode shape. A tip-sample interaction force can be approximated with a linear spring (capacitor) and damping resistor.

\subsubsection{Electrical Domain - Quasi-Static Model}

The piezoresistors respond to capacitive coupling and ground bounce in the electrical domain, temperature from the thermal domain, and strain from actuation and tip loading in the mechanical domain. The quasi-static model applies to DC and AC conditions when the beam is not in mechanical resonance. Modeling of the piezoresistor in the electrical domain is accomplished with separate resistors that experience feedback from the thermal and mechanical domains. The initial value of the resistor is $R_{0}$. The value of the resistor that captures thermal effects is:

$$
R_{t h}=R_{0} \alpha_{t h} \Delta T
$$

where $\alpha_{t h}$ is the temperature coefficient of resistivity and $\Delta T$ is coupled from the thermal domain. The value of the resistor that captures strain is calculated by taking the average strain in the sensor, and multiplying by the piezoresistive coefficient:

$$
R_{\epsilon}=\frac{R_{0} \rho_{\sigma}\left(1+\alpha_{\rho_{\sigma}} \Delta T\right)}{L_{p z r} t_{p z r}} \int_{x_{0}}^{x_{0}+L_{p z r}} \int_{z_{0}}^{z_{0}+t_{p z r}} \epsilon_{x}(x, z) d z d x
$$

where $L_{p z r}$ is the length of the piezoresistor, $t_{p z r}$ is the thickness of the piezoresistor, and $\epsilon_{x}$ is the strain in the longitudinal direction $x$. Equation (4.20) assumes that the piezoresistance is linear, which is a valid assumption for small strains. The strain itself can be expressed in terms of the bending moments, whether they are due to residual stress, thermal stress, or point-loading:

$$
\epsilon_{x}(x, z)=\left(z_{c}-z\right) \frac{M_{y}(x)}{(E I)_{e f f, y}}
$$


where $z_{c}$ is the position of the neutral axis of the beam. The capacitance $C_{m p}$ between the metal routing layer and the polysilicon below it is calculated based on measured parameters provided by the foundry design kit. Alternatively, the value may be extracted directly from the layout within the Cadence design environment. In many cases, the routing of the signal from the piezoresistor is in close proximity to other signal lines, which adds another parasitic capacitance that may also be extracted from layout. The electrical domain representation of a piezoresistive strain sensor is shown in Figure 34.

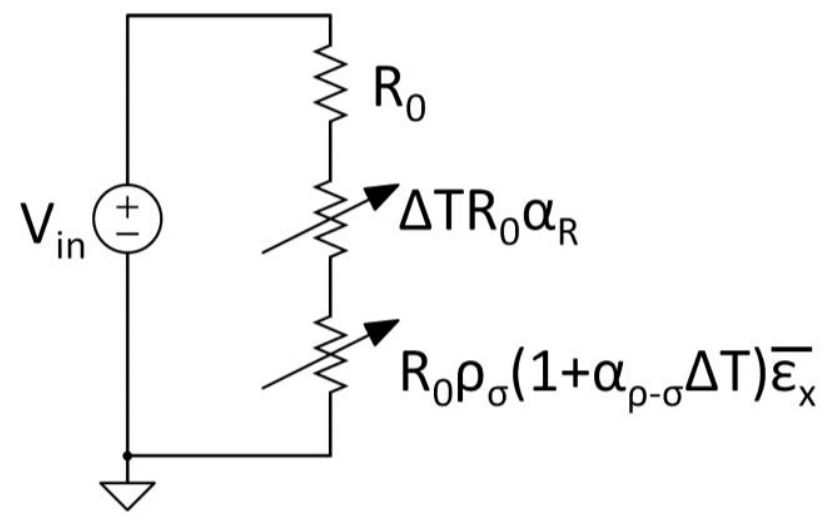

Figure 34: Electrical Domain Representation of a Piezoresistor

\subsubsection{Voltage or Current Drive - Quasi-Static Case}

Thus far, it has been shown that the static model of a piezoresistive strain sensor includes feedback from both the mechanical and the thermal domains, to the electrical domain. In addition, there are feedforward paths from the electrical domain to the thermal domain (through joule heating), and from the thermal domain to the mechanical domain (through thermal expansion of the bimorph). It is interesting to note that static quantities in the mechanical domain may therefore indirectly influence the temperature of the sensor, which is elucidated in the following scenario.

Consider a cantilever with an integrated piezoresistive strain sensor that has a positive coefficient of piezoresistivity. When the cantilever experiences static strain from tip-loading (a mechanical domain effect), the resistance of the sensor is increased. With constant-voltage drive, this implies that less power will be dissipated in the sensor, which in turn cools down the bimorph. This new, lower temperature may result in several problematic effects. First of all, the bimorph's deflection will decrease due to its lowered thermal moment, resulting in a possible tip-crash. Second of all, the lower bending moment reduces the strain in the beam, which counters the signal from the tip-sample interaction, thereby reducing the sensitivity of the instrument. The severity of these problems is 
compounded by the cooling of the bimorph due to tip-sample heat transfer. When the bimorph is cooled, its deflection decreases; this increases the likelihood of a tip crash. In addition, if the temperature coefficient of resistivity is also positive, the cooling will increase electrical resistance and reduce power even further. If, instead, the sensor is driven with a constant current source, these problems are mitigated. In fact, the use of a current source results in positive feedback and provides some gain to the tip-sample strain signal. Of course, the current must be limited to prevent thermal runaway, but this requirement is aligned with the general objective of minimizing self-heating, in light of the negative temperature coefficient of piezoresistivity. This example illustrates that the sign and magnitude of $\alpha_{t h}$ and $G F_{l}$ are of importance to the performance of the system, and that the type of source may be judiciously chosen to counter problematic feedback effects. The insight provided by this model is useful when interpreting results of contact-mode AFM.

\subsubsection{Thermal Domain}

The temperature distribution in a polysilicon strain sensor is the result of a combination of self-heating and thermal coupling from other parts of the instrument. In the thermal domain, the sensor and the flexure within which it is located must be connected in parallel, since it is assumed that the small crosssectional area of the beam is isothermal. A thermal model similar to the finite difference model developed for lateral actuators may be used (section 3.3.2), with the addition of source terms that represent self-heating. Alternatively, the transmission line model developed in 3.4.3 may be used, if the precise time and position dependence of temperature is required. In both cases, the thermal model of the flexure must be partitioned such that it can be connected to nodes in the sensor model. In Figure 35 below, the finite difference approximation is used. The heat current from the piezoresistor is injected into the flexure, and the temperature distribution from the flexure model is connected in parallel with the piezoresistor. Since the piezoresistor is embedded within the flexure beam, convective cooling may be ignored. Instead, the temperature nodes of the piezoresistor are directly connected to the temperature nodes in the flexure model, which are connected to convection resistors. Also, because the sensor is designed to operate at the mechanical resonant frequency of the beam, several nodes are present in the model to capture the dynamic temperature dependence along the length of the beam. To calculate the rate of local heat generation in the piezoresistor, the heat current source computes electrical power using the local resistance and the current from the electrical domain. To obtain the local resistance value, the electrical domain representation must also be partitioned into several seriesconnected resistors. 


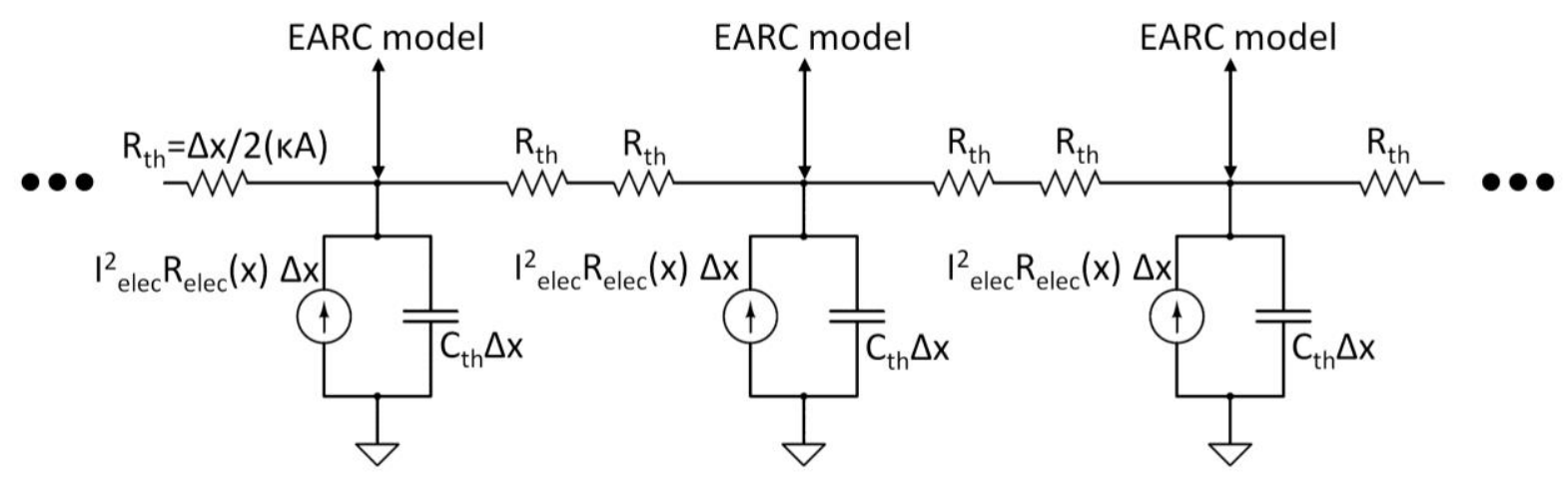

Figure 35: Thermal Model of a Piezoresistor

\subsubsection{Mechanical Domain - Quasi-Static Model}

The polysilicon strain sensor is embedded within a flexure, so the strain that is experiences can be obtained from the mechanical model of the flexure. Although the flexure itself does not contain a heater resistor, its cross-section is that of a bimorph, so a thermal moment exists. Other moments that contribute to strain in the cantilever are the moment from residual stress, and the moment from tipsample interaction. Since the moments are known from the vertical bimorph models, and are present as voltage sources in therein, it is convenient to substitute the individual moments into (4.21) to calculate the average strain in the piezoresistor:

$$
\epsilon_{x_{-} a v g}=\frac{1}{L_{p z r} t_{p z r}} \int_{x_{0}}^{x_{0}+L_{p z r}} \int_{z_{0}}^{z_{0}+t_{p z r}} \frac{\left(z-z_{c}\right)}{(E I)_{e f f, y}}\left[M_{y, r e s}(x)+M_{y, t h}(x)+M_{y, F_{t-s}}(x)\right] d z d x
$$

where $M_{y, F_{t-s}}(x)$ is the moment due to an applied force at the tip (tip-sample force), which can be expressed as:

$$
M_{y, F_{t-s}}(x)=F_{t-s}\left(L_{c a n t}-x\right)
$$

where $L_{\text {cant }}$ is the length of the cantilever (flexure) and the coordinates in are taken with respect to the beam origin, such that the placement of the piezoresistor within the flexure influences the strain in the sensor. Each of the contributing moments is available as a voltage source, so it is useful to separately evaluate their strain contributions individually, and then sum them to obtain the total strain. The average strain from the constant residual moment is: 


$$
\begin{gathered}
\epsilon_{x_{a v g}, r e s}=\frac{M_{y, r e s}}{t_{p z r}} \int_{z_{0}}^{z_{0}+t_{p z r}} \frac{\left(z-z_{c}\right)}{(E I)_{e f f, y}} d z=\frac{M_{y, r e s}}{(E I)_{e f f, y}}\left[t_{p z r}+2 z_{0}-2 z_{c}\right] \\
=M_{y, \text { res }} K_{\text {res }}
\end{gathered}
$$

Since the residual moment is constant, there is no need to include a dependent variable in the longitudinal $(x)$ direction.

The average strain from the thermal moment, including a thermal gradient, is:

$$
\begin{gathered}
\epsilon_{x_{a v g}, t h}=\frac{1}{L_{p z r} t_{p z r}} \int_{x_{0}}^{x_{0}+L_{p z r}} \int_{z_{0}}^{z_{0}+t_{p z r}} \frac{\left(z-z_{c}\right)}{(E I)_{e f f, y}} M_{0}\left[1+m_{t h} x\right] d z d x \\
=\frac{M_{0, t h} L_{p z r}}{(E I)_{e f f, y}}\left[-t_{p z r}-2 z_{0}+2 z_{c}\right]\left(1+m_{t h} x_{0}+\frac{m_{t h} L_{p z r}}{2}\right) \\
=M_{0, t h} K_{t h}
\end{gathered}
$$

The average strain from the tip-sample interaction force is:

$$
\begin{gathered}
\epsilon_{x_{a v g}, t-s}=\frac{1}{L_{p z r} t_{p z r}} \int_{x_{0}}^{x_{0}+L_{p z r}} \int_{z_{0}}^{z_{0}+t_{p z r}} \frac{\left(z-z_{c}\right)}{(E I)_{e f f, y}} F_{t-s}\left(L_{c a n t}-x\right) d z d x \\
=\frac{F_{t-s}}{2(E I)_{e f f, y}}\left[t_{p z r}+2 z_{0}-2 z_{c}\right]\left(2 L_{c a n t}-2 x_{0}-L_{p z r}\right) \\
=F_{t-s} K_{t-s}
\end{gathered}
$$

Equations (4.24)-(4.26) express the average strain in terms of geometric parameters and quantities that are available from the mechanical model of the flexures (constants that are lumped into $K_{r e s}, K_{t h}$, and $\left.K_{t-s}\right)$, and a single source term that corresponds to a voltage source (also from the flexure model). Since the moments and forces are already represented as voltage sources elsewhere, there is no need to incorporate them or the capacitors that they feed into the piezoresistor model.

Strain is a dimensionless quantity and its value in this model is used only to adjust the value of the electrical resistor. It is convenient to express strain as a voltage, because a voltage-controlled resistor is easy to implement in Spice-like solvers. The voltage sources corresponding to the moments and forces are scaled by the gains $K_{r e s}, K_{t h}$, and $K_{t-s}$ from (4.24)-(4.26), and then summed. This value is then used to adjust the resistor in the electrical domain, which will be shown in the complete model of the sensor. The quasi-static mechanical domain model of the piezoresistor is shown in Figure 36 . This model is 
easily partitioned to match the other models if the local piezoresistive change is required. In the partitioned model, the residual strain term would remain constant, the thermal moment would be applied from the corresponding segment in the thermal domain, and the tip-sample moment would come from the mechanical domain, in which the local voltage sources are scaled according to their distance from the tip.

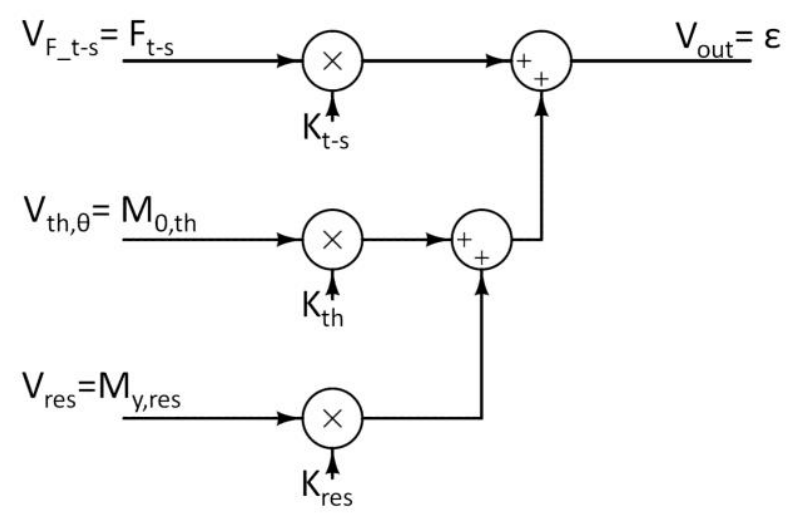

Figure 36: Strain in a Piezoresistor Represented as a Voltage

\subsubsection{Mechanical Domain - Dynamic Model}

When the flexure is in resonance, an additional source of strain dominates over the thermal, residual, and tip-sample force-related strains, in a properly designed system. This strain relates to the modeshape of the resonance of the flexure, and may be obtained from the displacement in the flexure's mechanical domain model.

The average strain may be obtained by evaluating equation (4.21) at the vertical position corresponding to the center of the sensor

$$
\epsilon_{x, a v g}(x, z)=\left(z_{c}-\frac{2 z_{0}+t_{p z r}}{2}\right) \frac{1}{L_{p z r}} \int_{x_{0}}^{x_{0}+L_{p z r}} \frac{M_{y, \omega r e s}(x)}{(E I)_{e f f, y}} d x
$$

Where $M_{y, r e s}(x)$ is the moment due to resonance. But it is known from equation (10.62) that:

$$
A \frac{M_{y, \omega r e s}(x)}{(E I)_{e f f, y}} \approx \frac{d^{2} w_{n}}{d x^{2}}
$$

where $A$ is the resonance amplitude. Thus, the second derivative of the mode shape may be substituted into the integral of equation (4.27). 


$$
\frac{d^{2} w_{n}}{d x^{2}}=\frac{A \lambda_{n}^{2}\left[\cosh \left(\frac{\lambda_{n} x}{L_{\text {cant }}}\right)\left(2 C_{n} \cos \left(\frac{\lambda_{n} x}{L_{\text {cant }}}\right)+1\right)+\cos \left(\frac{\lambda_{n} x}{L_{\text {cant }}}\right)\right]}{2 L_{\text {cant }}^{2}}
$$

A plot of the strain in the flexure is shown in Figure 37 for the first mode, normalized to the length $L$.

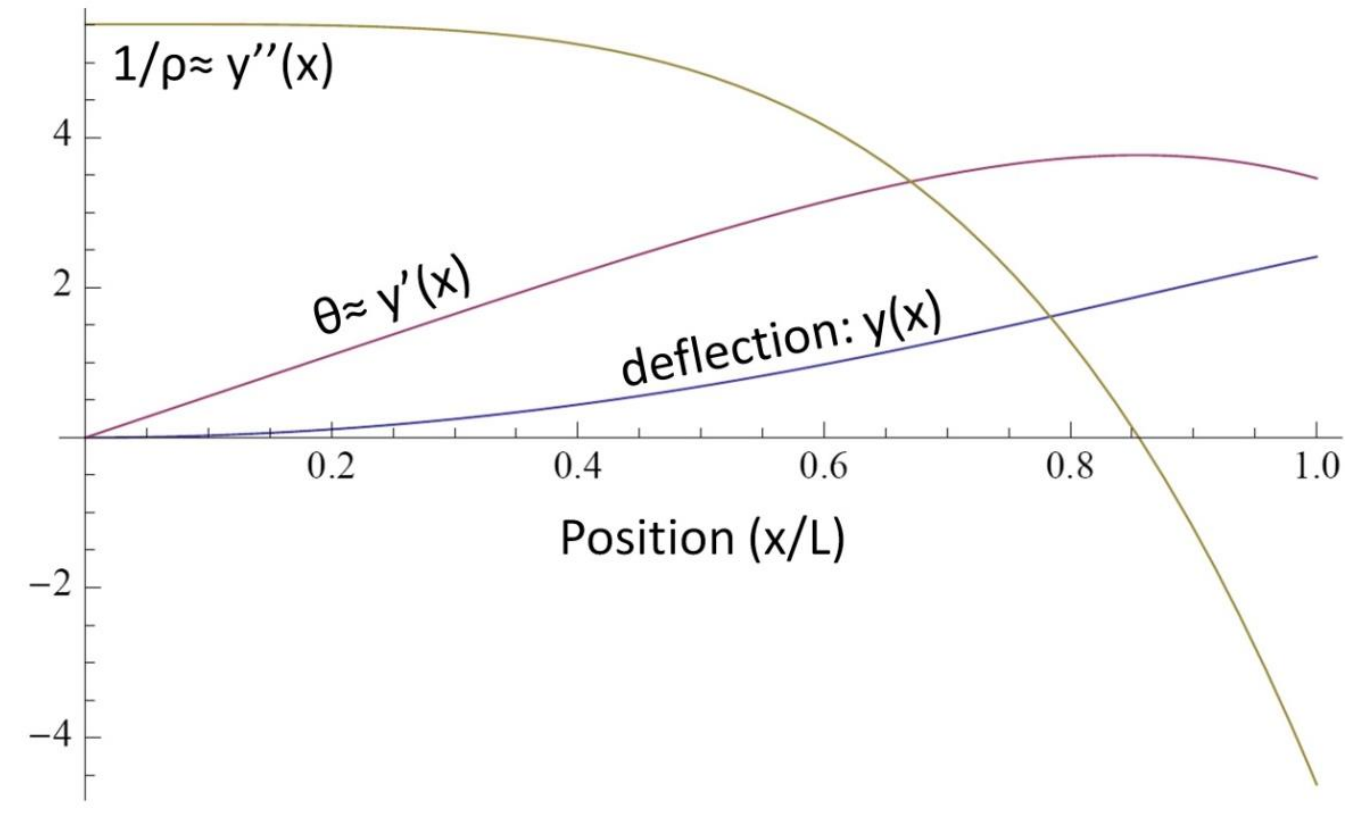

Figure 37: First Mode Shape and its Derivatives for a Resonant Cantilever

The piezoresistor is placed to capture the strain from resonance, which is almost constant for the first $1 / 3$ of the beam. Thus, the average value can be taken simply as the value at the center of the sensor in the longitudinal direction. That is:

$$
\epsilon_{x, \text { avg }}(x, z)=A\left(z_{c}-\frac{2 z_{0}+t_{p z r}}{2}\right) \frac{M_{y, \omega r e s}\left(\frac{x_{o}+L_{p z r}}{2}\right)}{(E I)_{e f f, y}}
$$

This is an important result, because it implies that the piezoresistor need not be placed at the base of the beam in order to preferentially capture the strain from resonance. A larger piezoresistor will produce less noise, while the strain remains close to its maximum value. In addition, the base of the actuator is where the parasitic thermal moment is highest, since the heat source is placed at the base of the beam for maximum efficiency. By offsetting the placement of the piezoresistor, one may increase immunity to the parasitic thermal signal to preferentially capture strain from resonance. This is in stark 
contrast to static AFM operation, where the moment (and strain) from tip-loading increases linearly and reaches a maximum at the base of the cantilever such that the piezoresistor must be placed where the temperature is highest.

\subsubsection{Electrical Domain - Dynamic Model}

Micromechanical resonators have been developed for electronic timing and filtering applications in part because their high Q-factors can provide good frequency stability, electrostatic actuation consumes little power, and batch fabrication offers lowered costs. For circuit designers, it is convenient to capture the resonant behaviour of the system exclusively in the electrical domain, using a lumped parameter model that does not need to explicitly reveal quantities in the mechanical or thermal domains. This circuit simplification is performed by incorporating the intra-domain coupling elements (for example, transformers in the case of electrostatic resonators) into the electrical lumped element values, which then become the "motional capacitance," "motional resistance," and "motional inductance." In [166], a 3-port model for a comb-drive resonator was developed using this method. In [157], a 1-port circuit model was developed for electrothermally actuated resonators with piezoresistive readout. In this section, a dynamic electrical model of is developed to facilitate the design of the electronics for dynamic AFM modes. In the model the EARC is considered as a flexural resonator with an integrated piezoresistive sensor that is excited through inertial forces by a vertical actuator with a separate resistive heater. There are two ports, one for the heating resistor of the actuator and another for the piezoresistor. Both the input voltage to the heater resistor port and its input impedance remain constant (aside from the TCR effect, which may be ignored for small signal AC analysis), while the piezoresistor port's complex impedance captures all the dynamics. In addition, there is no direct feedthrough in the electrical domain because the components are electrically isolated. To reduce the circuit to the electrical domain, the values of the lumped parameters are scaled to account for thermal feedthrough, thermal and mechanical dynamics, and the effect of the AC amplitude at the heater resistor input. The procedure for uncovering these values consists of determining the overall transfer function from input to output, one energy domain at a time, yielding an overall value for the transconductance of the circuit, $g_{m}$. Expressions for the $\mathrm{R}, \mathrm{L}$ and $\mathrm{C}$ values are then obtained as a function of $g_{m}$.

In the circuit, the vertical electrothermal actuator model from section 3.5 is connected to the flexural resonant cantilever model from section 4.1, which is in turn connected to the piezoresistor model. The AC deflection at the tip of the actuator is applied as a body force to the cantilever, which is assumed to 
be in resonance. A DC input applied to the piezoresistor terminals will therefore give rise to an $A C$ current, modulated by piezoresistor.

The electrical to thermal transfer function of the actuator may be expressed in the Laplace domain as:

$$
H_{t h}(s)=\frac{T_{a c}(s)}{V_{a c}(s)}=\frac{R_{t h}}{R_{0}\left(1+R_{t h} C_{t h} s\right)}
$$

where the square root circuit has been used to linearize the system, and it is assumed that a DC bias offsets the $\mathrm{AC}$ voltage to a positive value. The temperature to vertical displacement transfer function may be derived from the simplified circuit below in Figure 38.

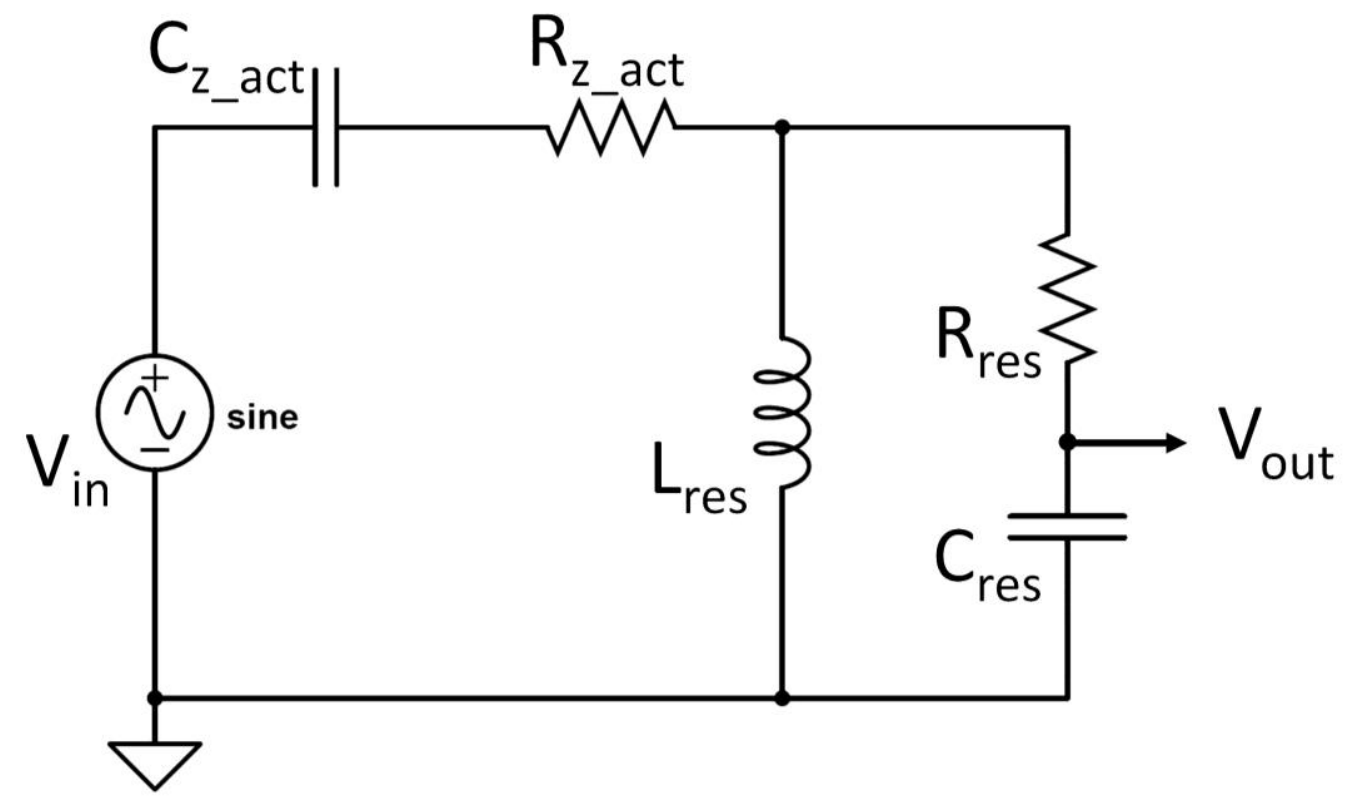

Figure 38: Electrical representation of an EARC

The force generated by the thermal moment $\left(V_{i n}\right)$ is shared between the deflection of the vertical actuator and the acceleration of the mass of the resonator, so they are connected in series in the $e \rightarrow V$ convention. On the other hand, since the inertial forces of the resonator (and the load mass) are deflecting the spring, these components are connected in parallel. The scaling factor from temperature to force is related to the material properties and geometry of the actuator, as discussed in section 3.5.3 for the case of vertical bimorphs. Since the linear deflection of the actuator is of interest, and the force should be referred to the tip, equation (4.31) may be scaled using equation (10.142) to obtain the transfer function from input voltage to output force: 


$$
H_{V-F}(s)=\frac{V_{t h}(s)}{T_{a c}(s)} \frac{T_{a c}(s)}{V_{a c}(s)}=\frac{2 S_{M_{\_} y, t h}}{L} \frac{R_{t h}}{R_{0}\left(1+R_{t h} C_{t h} s\right)}
$$

Several observations can be made about this circuit and how it relates to device performance. The goal of the design is for the actuator to produce a static deflection to track topology, while providing a dynamic deflection to excite the resonator. Thus, the DC voltage of the force source is used exclusively to deflect the actuator (by charging its capacitance), and no current flows into the resonator. At resonance, some $A C$ current flows through the actuator, and the resulting charge in the resonator's capacitance represents the instantaneous tip deflection. The resonance of the two systems should be decoupled to avoid the excitation of unwanted vibrations in the actuator, and to minimize energy loss from the resonator to the rest of the instrument. Intuitively, if the actuator has a high stiffness-toweight ratio (high resonant frequency) and the resonator is designed to have a low resonant frequency, the two resonators will be undercoupled. The effect of a stiff actuator, represented by a small capacitor, is to attenuate the transfer functions of both components while reducing the loading on the resonator, as shown in Figure 39 below.
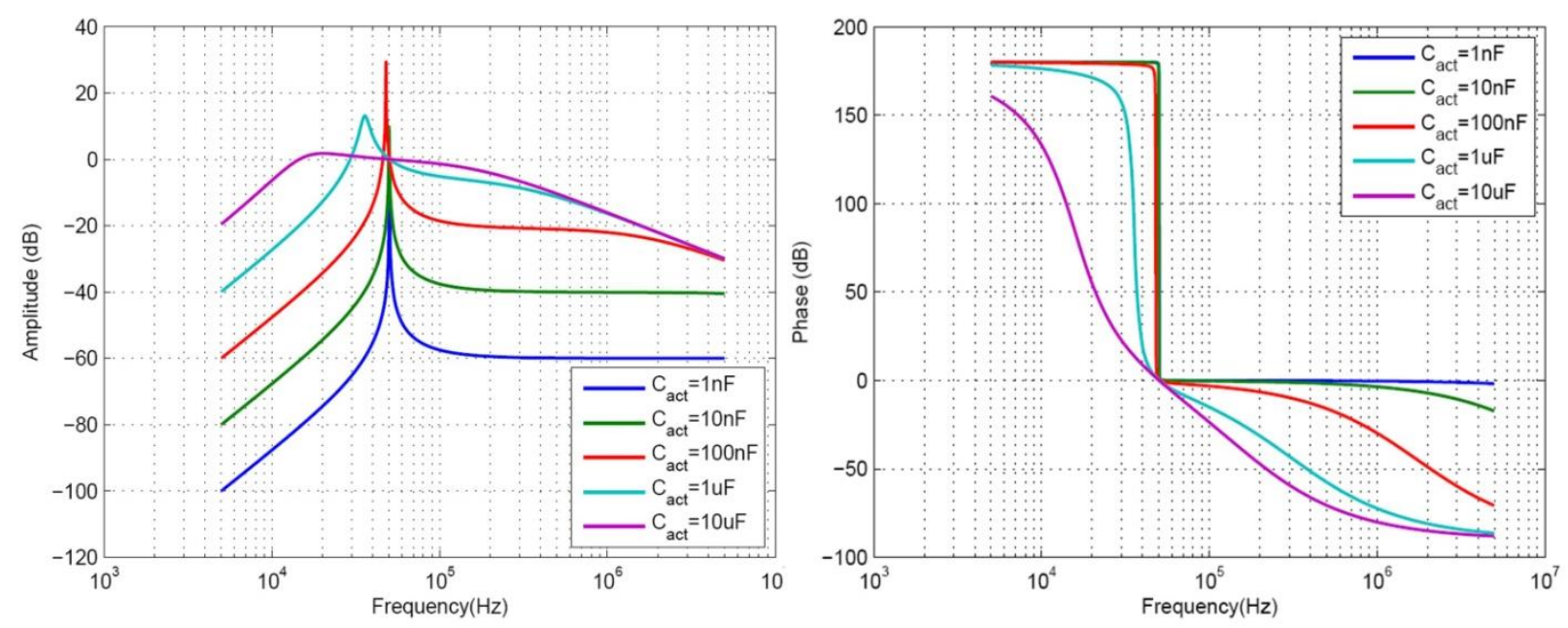

Figure 39: Loading Effect of Vertical Actuator on EARC Frequency Response

In order to compute the transfer function from the thermal domain to the mechanical domain, the resonant frequency is first calculated. At resonance, a coupling factor is introduced to capture the effect of the impedance mismatch between the actuator and resonator, which is desirable in this case because a matched impedance would cause the actuator to vibrate along with the resonator. The charge on the resonator's capacitance is then calculated. 
The resonant frequency may be calculated by setting the imaginary part of the admittance to zero as follows:

$$
\begin{gathered}
Y_{\text {res }}=\frac{1}{j \omega L}+\left(j \omega C \| \frac{1}{R}\right)=\frac{j \omega C+\frac{1}{R}-\frac{\omega^{2} C L}{R}}{j \omega \frac{L}{R}-\omega^{2} L C} \times \frac{j \omega \frac{L}{R}+\omega^{2} L C}{j \omega \frac{L}{R}+\omega^{2} L C} \\
\operatorname{Im}\left[\left(j \omega C+\frac{1}{R}-\frac{\omega^{2} C L}{R}\right) \times\left(j \omega \frac{L}{R}+\omega^{2} L C\right)\right]=0 \\
\omega_{0}=\frac{1}{\sqrt{L C-R^{2} C^{2}}}
\end{gathered}
$$

As the admittance of the circuit approaches zero, the remaining real component the impedance is scaled upwards. A series-shunt transformation of the capacitive branch of the circuit may be performed at this point. The equivalent values of the parallel resistance and capacitance are:

$$
R_{r e s, \|}=R_{r e s}\left(1+Q^{2}\right) \quad C_{r e s, \|}=C_{r e s}\left(1+Q^{-2}\right) \approx C_{r e s}
$$

The Q-factor of the resonator may be calculated as:

$$
Q=R_{r e s, \|} \sqrt{\frac{C_{r e s}}{L_{r e s}}}=R_{r e s}\left(1+Q^{2}\right) \sqrt{\frac{C_{r e s}}{L_{r e s}}} \approx \frac{1}{R_{r e s}} \sqrt{\frac{L_{r e s}}{C_{r e s}}}
$$

Where it was assumed that $Q^{2} \gg 1$. At resonance, only the parallel resistance remains as the load to the circuit, and the voltage across the resonator may be obtained with:

$$
\left.V_{r e s}\right|_{j \omega_{0}}=\left.\frac{\left.Z_{r e s}\right|_{j \omega_{0}}}{\left.Z_{r e s}\right|_{j \omega_{0}}+\left.Z_{a c t}\right|_{j \omega_{0}}} V_{i n}\right|_{j \omega_{0}}=\left.\frac{R_{r e s, \|}}{R_{r e s, \|}+\left.Z_{a c t}\right|_{j \omega_{0}}} V_{i n}\right|_{j \omega_{0}}
$$

Where the subscript res corresponds to resonator quantities and the subscript act is for actuator quantities. The coupling factor may be expressed as:

$$
\eta=\left|\frac{V_{r e s}}{V_{\text {in }}}\right|_{j \omega_{0}}|=| \frac{R_{r e s, \|}}{R_{r e s, \|}+j \omega_{0} L_{a c t}+\frac{1}{j \omega_{0} C_{a c t}}+R_{a c t}} \mid
$$

Assuming that the actuator has a high stiffness to weight ratio and neglecting the small series damping, the coupling factor may be simplified: 


$$
\eta \approx \sqrt{\frac{R_{r e s, \|}^{2}}{R_{r e s, \|}^{2}+\left(\frac{1}{\omega_{0} C_{a c t}}\right)^{2}}}=\frac{Q \sqrt{\frac{L_{r e s}}{C_{r e s}}}}{\sqrt{Q^{2} \frac{L_{r e s}}{C_{r e s}}+\left(\frac{1}{\omega_{0} C_{a c t}}\right)^{2}}}
$$

To limit the ac deflection of the actuator, the coupling factor should be increased by decreasing $C_{a c t}$ (i.e., increasing the spring constant $k_{a c t}$ ). This has the desired effect of pushing the pole of the actuator's transfer function to a higher frequency, while attenuating both the transfer functions of the actuator and the resonator.

To continue with the derivation of the transfer function from temperature to resonator deflection, the charge on the resonator's capacitor is calculated. The voltage across the resonator is obtained using $R_{r e s, \|}$ as the impedance of the resonator, in series with the actuators compliance. This voltage is multiplied by $Q$ to obtain the resonant current, and the current is integrated (divided by $s$ ) to obtain the charge $q_{\text {res }}$. The voltage across the resonator is:

$$
V_{r e s}(s)=\frac{R_{r e s, \|}}{R_{r e s, \|}+\frac{1}{s C_{a c t}}} V_{t h}(s)
$$

The current through the capacitor is:

$$
I_{C_{\text {res }}}(s)=Q V_{\text {res }}(s)
$$

Finally, the charge on the capacitor is:

$$
q_{C_{r e s}}(s)=\frac{I_{C_{r e s}}(s)}{s}
$$

The transfer function from input force to resonant displacement may be expressed in terms of the Qfactor, using equation (4.37).

$$
H_{F-Z}(s)=\frac{q_{C_{r e s}}(s)}{V_{t h}(s)}=\frac{Q}{s} \frac{R_{r e s, \|}}{R_{r e s, \|}+\frac{1}{s C_{a c t}}}=\frac{Q^{2} \sqrt{\frac{L_{r e s}}{C_{r e s}}}}{Q \sqrt{\frac{L_{r e s}}{C_{r e s}}} s+\frac{1}{C_{a c t}}}
$$

The charge on the capacitor represents the amplitude of the resonant motion, which relates to the strain in the beam. To return to the electrical domain, the transfer function from deflection to 
resistance is calculated. The strain in the piezoresistor is calculated using equation (4.30), substituting the charge on the capacitor for the amplitude:

$$
\epsilon_{x}(s)=q_{C_{r e s}}(s)\left[\left(z_{c}-\frac{2 z_{0}+t_{p z r}}{2}\right)\left(\frac{M_{y, r e s}\left(\frac{x_{o}+L_{p z r}}{2}\right)}{(E I)_{e f f, y}}\right)\right]
$$

Where the function $M_{y, \text { res }}$ is evaluated for the first mode shape of the beam, at the center of the piezoresistor. The term in brackets may be thought of as a strain sensitivity shape factor $S_{\epsilon, n=1}$ that scales the strain based on the first mode shape of the beam. Equation (4.45) may be written compactly as:

$$
\epsilon_{x}(s)=q_{C_{r e s}}(s) S_{\epsilon, n=1}
$$

The piezoresistance of the device is used to transfer these quantities back into the electrical domain:

$$
R_{\epsilon}(s)=\epsilon_{x}(s) R_{0} \rho_{\sigma}
$$

If a DC bias is applied to the piezoresistor, the current flowing through it is the motional current:

$$
i_{m}(s)=\frac{V_{\text {bias }}}{R_{0}} R_{\epsilon}(s)
$$

The transfer function from the resonant deflection to the motional current is:

$$
H_{Z-I}(s)=\frac{i_{m}(s)}{q_{C_{\text {res }}}(s)}=V_{\text {bias }} \rho_{\sigma} S_{\epsilon, n=1}
$$

The total transfer function, from the input voltage of the actuator to the output current of the piezoresistor is called the motional admittance $Y_{m}$ of the device. Multiplying all of the transfer functions:

$$
H_{T}(s)=\frac{i_{m}(s)}{V_{a c}(s)}=Y_{m}=H_{V F} H_{F Z} H_{Z I}
$$

Expanding the terms: 


$$
Y_{m}(s)=\frac{2 S_{M_{\_} y, t h} V_{\text {bias }} \rho_{\sigma} S_{\epsilon, n=1}}{L_{\text {res }} R_{0}} \times \frac{R_{t h}}{\left(1+R_{t h} C_{t h} s\right)} \times \frac{Q^{2} \sqrt{\frac{L_{r e s}}{C_{r e s}}}}{Q \sqrt{\frac{L_{r e s}}{C_{\text {res }}}} s+\frac{1}{C_{a c t}}}
$$

The middle term in this expression accounts for thermal rolloff, which occurs at a much lower frequency than the resonant frequency of the flexure. The inverse of the thermal time constant of the actuator is a much smaller quantity than the natural frequency of the resonator: $\tau^{-1}=\left(R_{t h} C_{t h}\right)^{-1} \ll \omega_{0}$. For example, a typical vertical actuator used in this work may have a thermal time constant of $\sim 2-3 \mathrm{~ms}$, while the mechanical resonant frequency of the flexures is $\sim 20 \mathrm{kHz}$. Therefore, based on the fact that $1+R_{t h} C_{t h} S \approx R_{t h} C_{t h} S$, the following simplification can be made,

$$
Y_{m}(s)=\frac{2 S_{M_{\_} y, t h} V_{\text {bias }} \rho_{\sigma} S_{\epsilon, n=1}}{L_{\text {res }} R_{0} C_{t h} S} \frac{Q^{2} \sqrt{\frac{L_{\text {res }}}{C_{\text {res }}}}}{Q \sqrt{\frac{L_{\text {res }}}{C_{\text {res }}}} s+\frac{1}{C_{\text {act }}}}
$$

This simplification preserves the attenuation that is caused by operation beyond the thermal cut-off frequency. The motional impedance of the circuit is the inverse of the admittance:

$$
\left.Z_{m}(s)\right|_{s=j \omega_{0}}=\frac{-L_{r e s} R_{0} C_{t h} Q \sqrt{\frac{L_{r e s}}{C_{r e s}}} \omega_{0}^{2}+j L_{r e s} R_{0} C_{t h} \frac{1}{C_{a c t}} \omega_{0}}{2 S_{M_{-} y, t h} V_{\text {bias }} \rho_{\sigma} S_{\epsilon, n=1} Q^{2} \sqrt{\frac{L_{r e s}}{C_{r e s}}}}
$$

Separating the real and imaginary components:

$$
\begin{gathered}
\operatorname{Re}\left(Z_{m}\right)=\frac{-L_{r e s} R_{0} C_{t h} \omega_{0}{ }^{2}}{2 S_{M_{-} y, t h} V_{\text {bias }} \rho_{\sigma} S_{\epsilon, n=1} Q} \\
\operatorname{Im}\left(Z_{m}\right)=\frac{j L_{\text {res }} R_{0} C_{t h} \frac{1}{C_{a c t}} \omega_{0}}{2 S_{M_{-} y, t h} V_{\text {bias }} \rho_{\sigma} S_{\epsilon, n=1} Q^{2} \sqrt{\frac{L_{\text {res }}}{C_{\text {res }}}}}
\end{gathered}
$$

Based on these expressions, the real component of the impedance is a factor of $C_{a c t} \omega_{0} Q$ bigger than the imaginary component. Since $C_{a c t}{ }^{-1} \ll \omega_{0} Q$, the imaginary component of the impedance is negligible compared to the real component. Also, it can be observed that based on the sign of the piezoresistive coefficient, the small motional resistance may be positive or negative. A negative resistance with sufficient loop gain has been shown to sustain oscillation using a thermally actuated, 
piezoresistively transduced micromachined resonator [167]. In the present circuit, the negative resistance would have to surpass the value of $R_{0}$; thus far, self-sustained oscillation has not been observed in these devices.

The electrical domain model of the EARC with an integrated piezoresistive sensor, at resonance, is represented by the series RLC circuit in Figure 40 . The motional resistance $r_{m}$ is taken to be the real component of the motional impedance. The resonant frequency of the device is then accounted for by inserting a motional capacitance $c_{m}$ and a motional inductance $l_{m}$ with values that are chosen based on $Q$ and $\omega_{0}$, which may be measured. Optionally, a series inductor may be added to account for the small phase shift due to the imaginary component of the motional impedance from equation (4.55). Expressions for $r_{m}, l_{m}$, and $c_{m}$ are:

$$
\begin{aligned}
& r_{m}=\frac{-L_{r e s} R_{0} C_{t h} \omega_{0}^{2}}{2 S_{M_{-} y, t h} V_{\text {bias }} \rho_{\sigma} S_{\epsilon, n=1} Q} \quad l_{m}=\frac{Q r_{m}}{\omega_{0}} \quad c_{m}=\frac{1}{Q r_{m} \omega_{0}}
\end{aligned}
$$

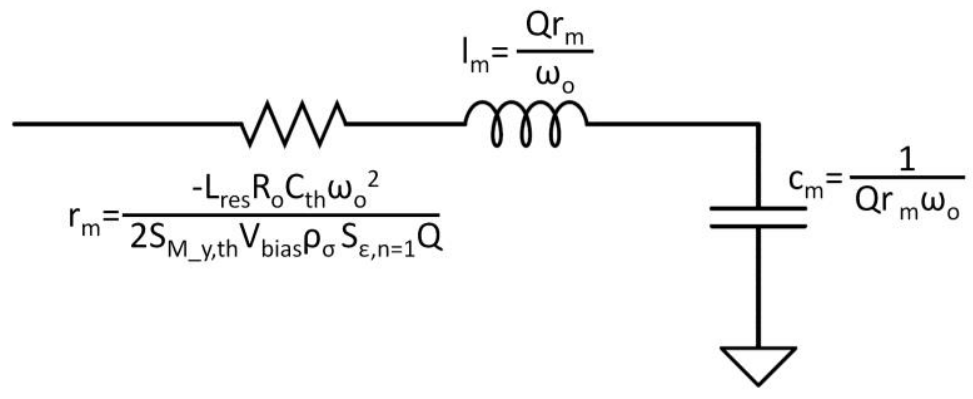

Figure 40: Motional Impedance of EARC

Without the square-root circuit present, the transfer function $H_{t h}(s)$ from equation (4.31) should be modified to include the square-law relationship between voltage and temperature. The power is proportional to temperature and follows:

$$
P_{a c}(\omega)=\frac{\left(V_{D C}+V_{A C} \sin (\omega t)\right)^{2}}{R_{0}}=V_{D C}{ }^{2}+2 V_{D C} V_{A C} \sin (\omega t)+V_{A C}{ }^{2} \sin ^{2}(\omega t)
$$

The modified transfer function only considers the AC term at the excitation frequency $\omega$ :

$$
H_{t h-b}(s)=\frac{T_{a c}(s)}{V_{a c}(s)}=\frac{2 V_{D C} V_{A C} R_{t h}}{R_{0}\left(1+R_{t h} C_{t h} s\right)}
$$


This transfer function is valid for a large DC offset and a small AC signal. Unfortunately, if used in the present configuration, the DC bias (which tracks topology) will modulate the resonant amplitude and contribute to image distortions and degraded controller performance. This issue is depicted below in Figure 41. If the AC input voltage is held constant, the vibration amplitude increases with the offset voltage.

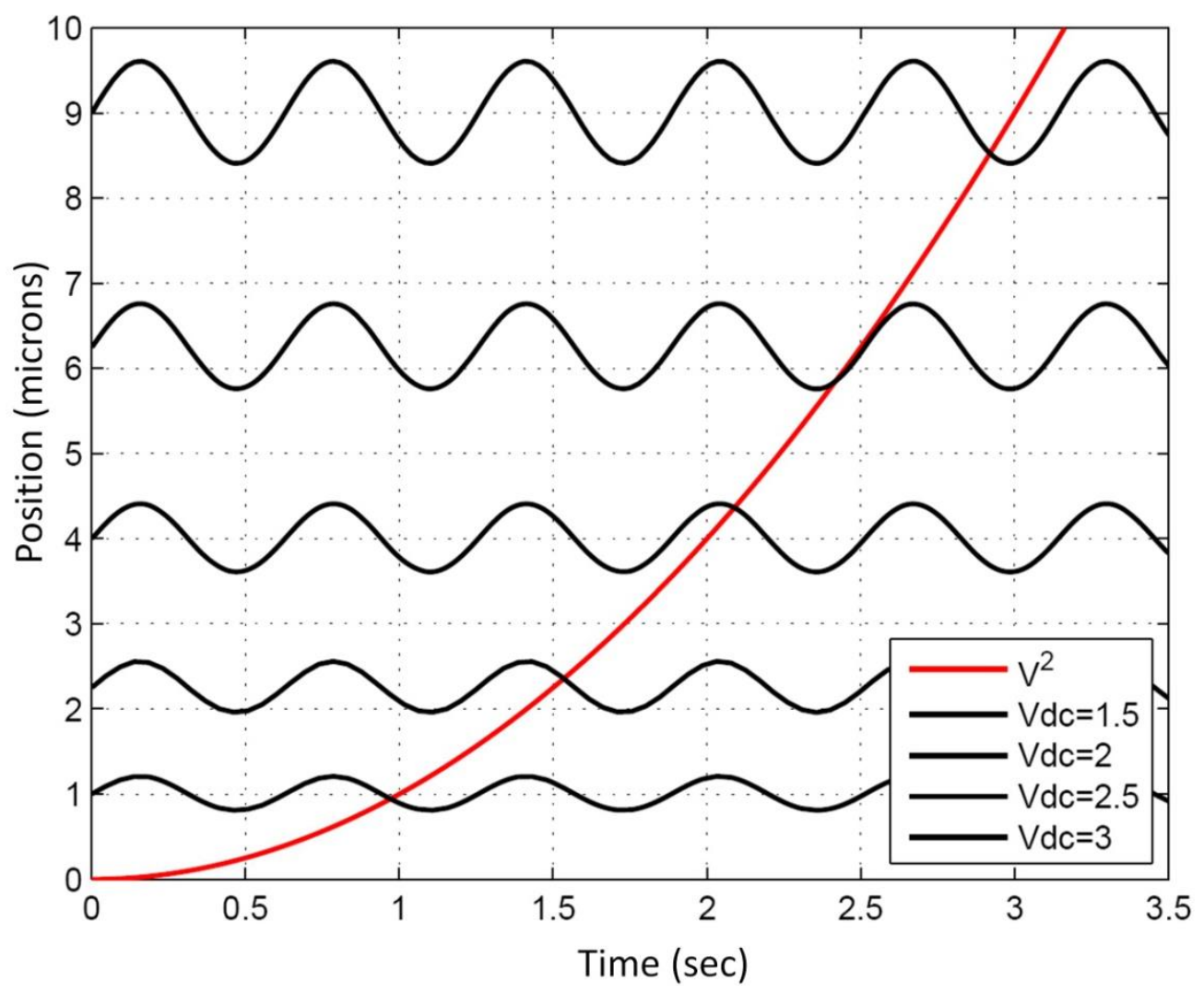

Figure 41: Modulation of the AC Amplitude of Vibration by a DC offset.

\subsection{Electromechanical and Mechanical Quality Factor Enhancement}

It is possible to drive the piezoresistor directly with an AC signal. In this case, the AC thermal actuation from self-heating is concentrated in the piezoresistor. A DC bias voltage or current may be added to increase the output signal if the piezoresistor is placed in a bridge configuration. The separate vertical actuator may be used to track topology while the piezoresistive bimorph is in resonance. This configuration is shown below in Figure 42, where the left half of the image is similar to the driving method for I2BAR devices reported in [168]. Because this method does not correspond to either the SARC (in which the piezoresistor also acts as a vertical actuator) or the EARC (in which the piezoresistor only has a DC bias across it), it is referred to here as a hybrid mode. One might think that in the direct- 
drive configuration, feedthrough from the excitation signal would combine with TCR effects to obscure the signal of interest. However, a unique Q-factor enhancement mechanism has been observed when operating in the SARC mode. Q-factors of $>50,000$ have been measured in air using this method; the same cantilevers, when excited using the EARC technique without a DC offset, showed measured Q's of only 50. The $Q$ increases with the applied DC bias up to a point, beyond which it drops. In addition, the amplitude of the AC component is not observed to adjust the $Q$ factor.

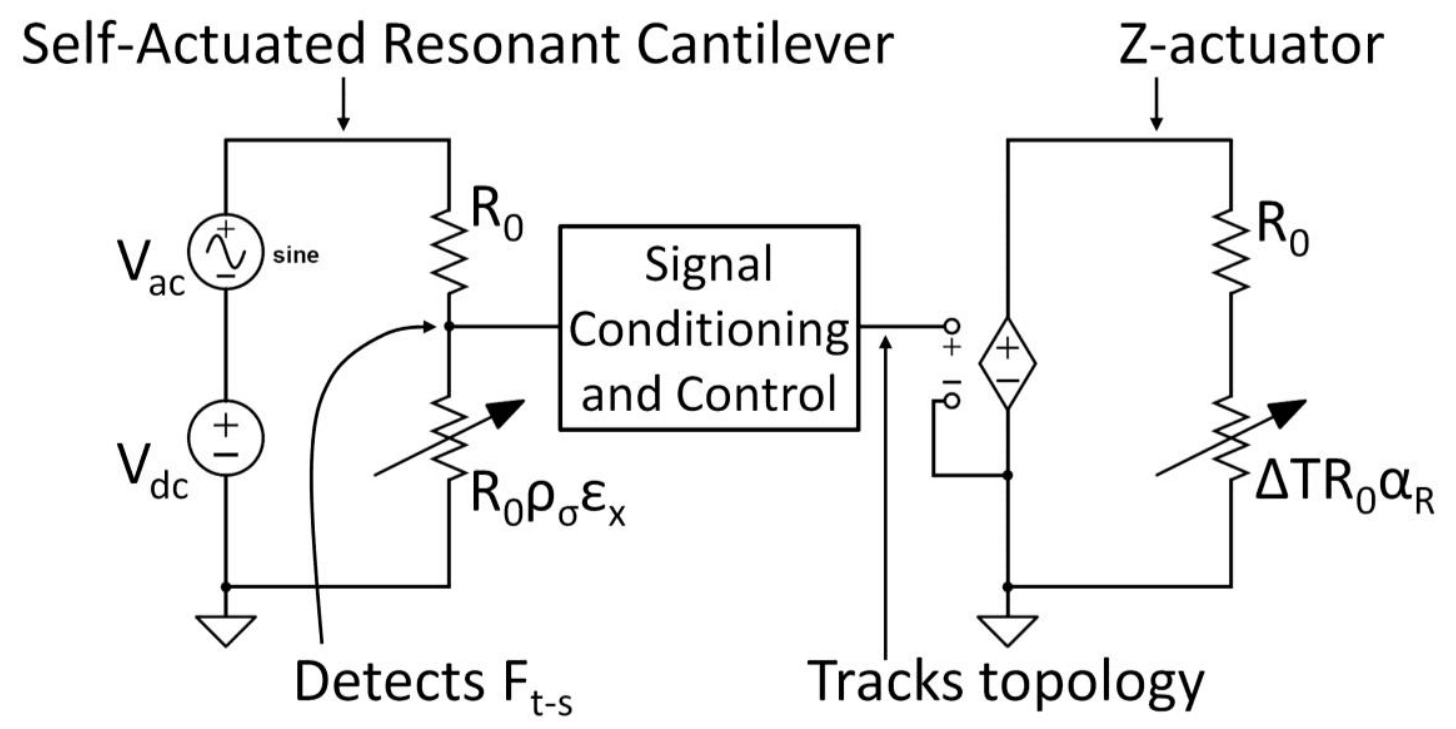

Figure 42: Hybrid Mode of Operation That Enhances Quality Factor

To measure the response of the cantilevers using piezoresistive detection, a Wheatstone bridge configuration is used. The sense and balance resistors are located in different branches of the bridge in order to mitigate ground bounce caused by vertical and lateral actuators that may share a common path to the ground pin. The bridge configuration is shown below in Figure 43.

Several mechanisms are believed to contribute to the enhanced $Q$ measured in the hybrid mode of operation. The dominant mechanism is the result of localized matching in the bridge circuit that occurs near the resonant frequency, when both sides of the bridge share the same phase value. Although this property improves the dynamic range of the measurement, it does not improve the sensitivity of the cantilever (minimum detectable force). Another mechanism is Q-factor enhancement under driving force control, which has been used in the AFM community (where it is known as Q-control [169]) to improve tip-sample interaction force resolution. In this method, a signal that is opposite to the damping term in the equation of motion of the resonator (negative damping) is injected along with the driving 
force. Precise control of the phase of this additional signal is required to ensure that it compensates the damping, which is proportional to velocity (90 degrees out-of-phase with position). A position sensor is often used to measure the resonator's motion and generate the Q-control signal.

\section{Reactive Wheatstone Bridge}

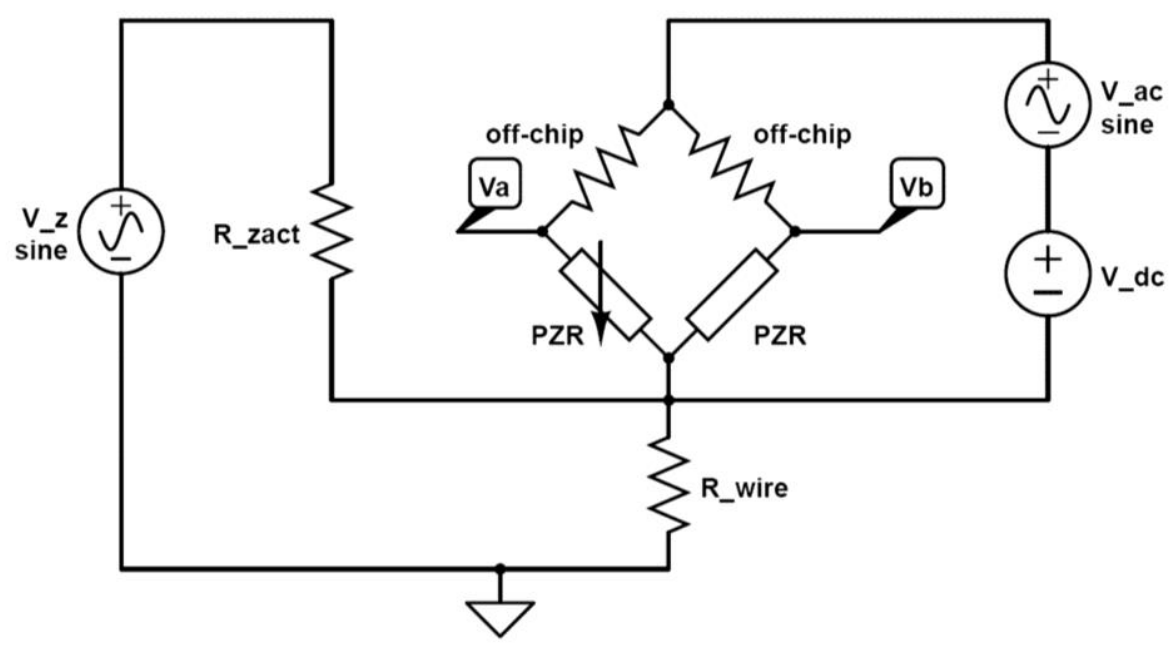

Figure 43: Configuration of piezoresistive bridge circuit used for dynamic operation of cantilevers

Parametric pumping, a well-known non-linear dynamics phenomenon, is yet another way to enhance the Q-factor of a resonator. Parametric enhancement of the Q-factor of a cantilever was first demonstrated in the context of AFM in [170] and has been studied in the MEMS community [171], [172], [173]. Although the dynamics of the processes are well understood, the particular mechanisms contributing to the self-Q enhancement of SARC devices has not yet been fully investigated. Measurements indicate that higher harmonics are generated by thermal and piezoresistive modulation of the resistor value; however, this effect has not been isolated from the negative damping effect. To understand the proposed mechanisms, the dynamic mechanical and electrical models from section 4.2.5 and 4.2.6 are a good starting point.

The remainder of this chapter provides insight into Q-enhancement mechanisms based on measurements from the electrical and mechanical domains. 


\subsubsection{Electrical Domain Measurements}

Measurements in the electrical domain were performed with the use of a lock-in amplifier. The data reflects the nature of the interaction of electrical, thermal and mechanical effects combined with the transfer functions of the bridge configuration and instrumentation. To interpret measurements from the half-bridge configuration in Figure 43 , it is instructive to examine a measurement of a single piezoresistor under voltage excitation. This measurement is used to identify the quantities that influence the sensors' output. Once the model has been established, the frequency-dependent effects of the half-bridge circuit are examined.

Figure 44 is a simplified model that corresponds to the measurement of a single piezoresistor placed under a strain-sensing cantilever beam. A current source in the thermal domain represents self-heating in the device. Two nodes are present in the thermal domain, to separately capture the temperature of the piezoresistor and the temperature of the bimorph.

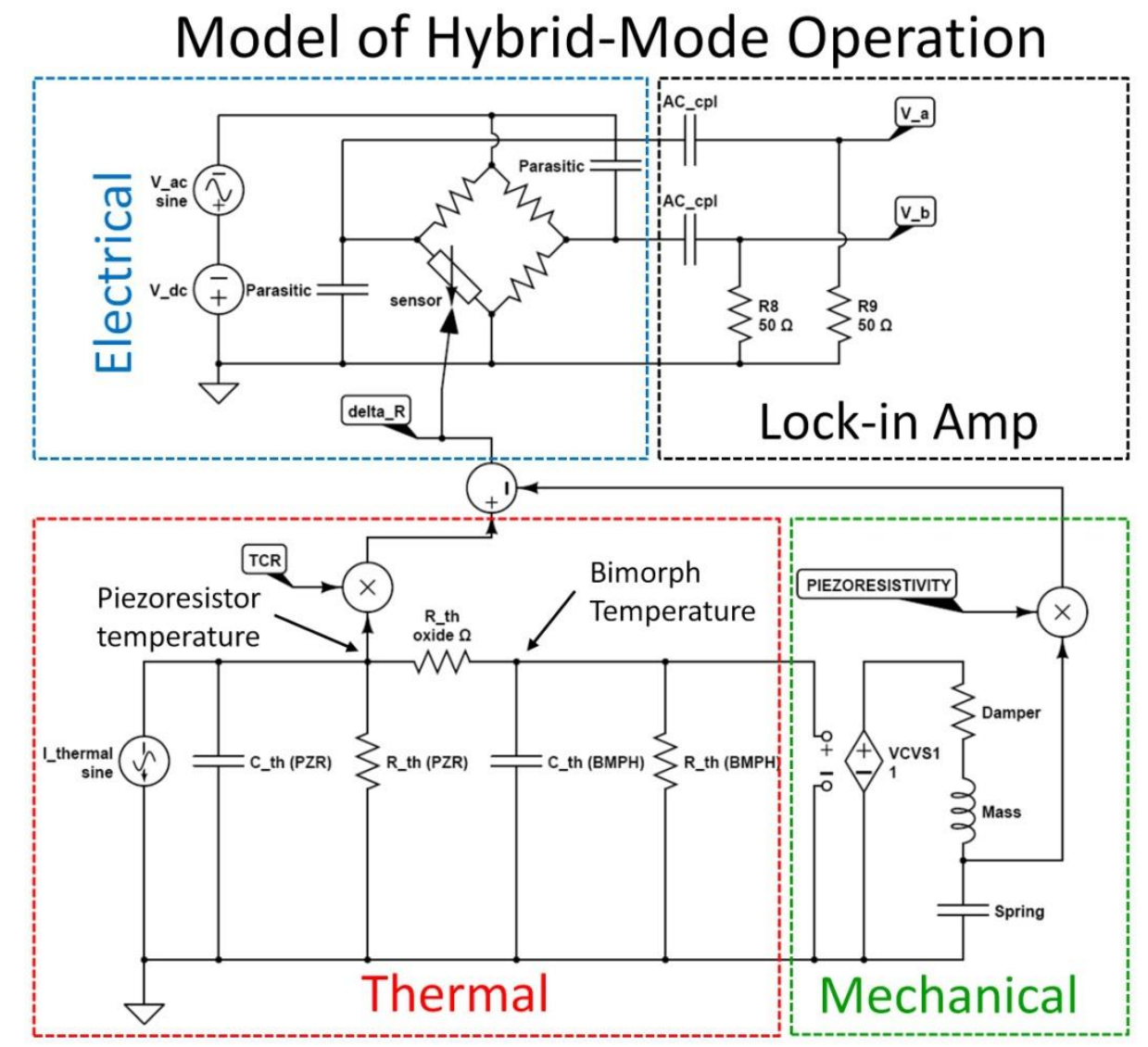

Figure 44: Measurements of hybrid-mode operation reflect quantities in the electrical, thermal and mechanical domains 
In Section 3.2.3 it was observed that for a bimorph in static operation, the piezoresistance may dominate over the TCR. In dynamic operation, there is a temperature gradient in the $\mathrm{SiO}_{2}$ between the piezoresistor and the Aluminum layer. The bimorph deflection depends on the temperature difference between the metal and dielectric layers, and gives rise to a strain-dependent modulation of the resistance. On the other hand, the temperature in the piezoresistor gives rise to a TCR-dependent modulation of the same resistor. In order to interpret the frequency-dependent phase and amplitude response of the device, the model must separately capture the effects of strain and temperature. The temperature of the resistor is scaled by the TCR, and the strain in the bimorph (proportional to deflection) is scaled by the piezoresistive coefficient. Since the two contributions are opposite in direction, the difference in the quantities is applied to the electrical resistor. In the electrical domain, a Wheatstone bridge is used to measure the sensor's output resistance. The bridge is powered by a large DC signal and a small AC signal. In practise, the DC bias voltage is used to provide power to the resonant device, via modulation of its resistance through piezoresistive and TCR effects; effect of the DC signal is not captured by the Spice model, but its influence may be captured by varying parameters that are influenced by temperature and by adjusting the input thermal current. Parasitic capacitors are included to account for coupling to the the z-actuator signal and ground, which may occur in the scanner. The input stage of the lock-in amplifier is AC-coupled, as indicated in Figure 44. To capture the mechanical resonance, each mode may be represented as a series RLC combination, and multiple modes may be cascaded in parallel.

Figure 45 illustrates the output of the model at three nodes from the model. The temperature of the piezoresistor is represented as its voltage value in the thermal domain, scaled by the TCR. The strain in the piezoresistor is represented by its deflection in the mechanical domain, scaled by the piezoresistivity. The difference between these values represents the change in resistance that is applied to the electrical resistor in the bridge circuit. A low-frequency pole representing the thermal timeconstant of the bimorph occurs at $\sim 1 \mathrm{kHz}$, while a higher frequency pole representing the vertical time constant from the heater to the Aluminum top surface occurs at $\sim 50 \mathrm{kHz}$. At the resonance frequency $(\sim 140 \mathrm{kHz})$, the amplitude of the signal from piezoresistive modulation may surpass that of the thermal signal.

Figure 46 illustrates the frequency response of the signals at the inputs of the lock-in amplifier. The AC coupling capacitors at the inputs serve as low-pass filters, attenuating both signals from DC to a roll-off frequency at $\sim 1 \mathrm{kHz}$. The effect of parasitic capacitive coupling in the measurement setup has been 


\section{Bode Plots of Piezoresistor Values}
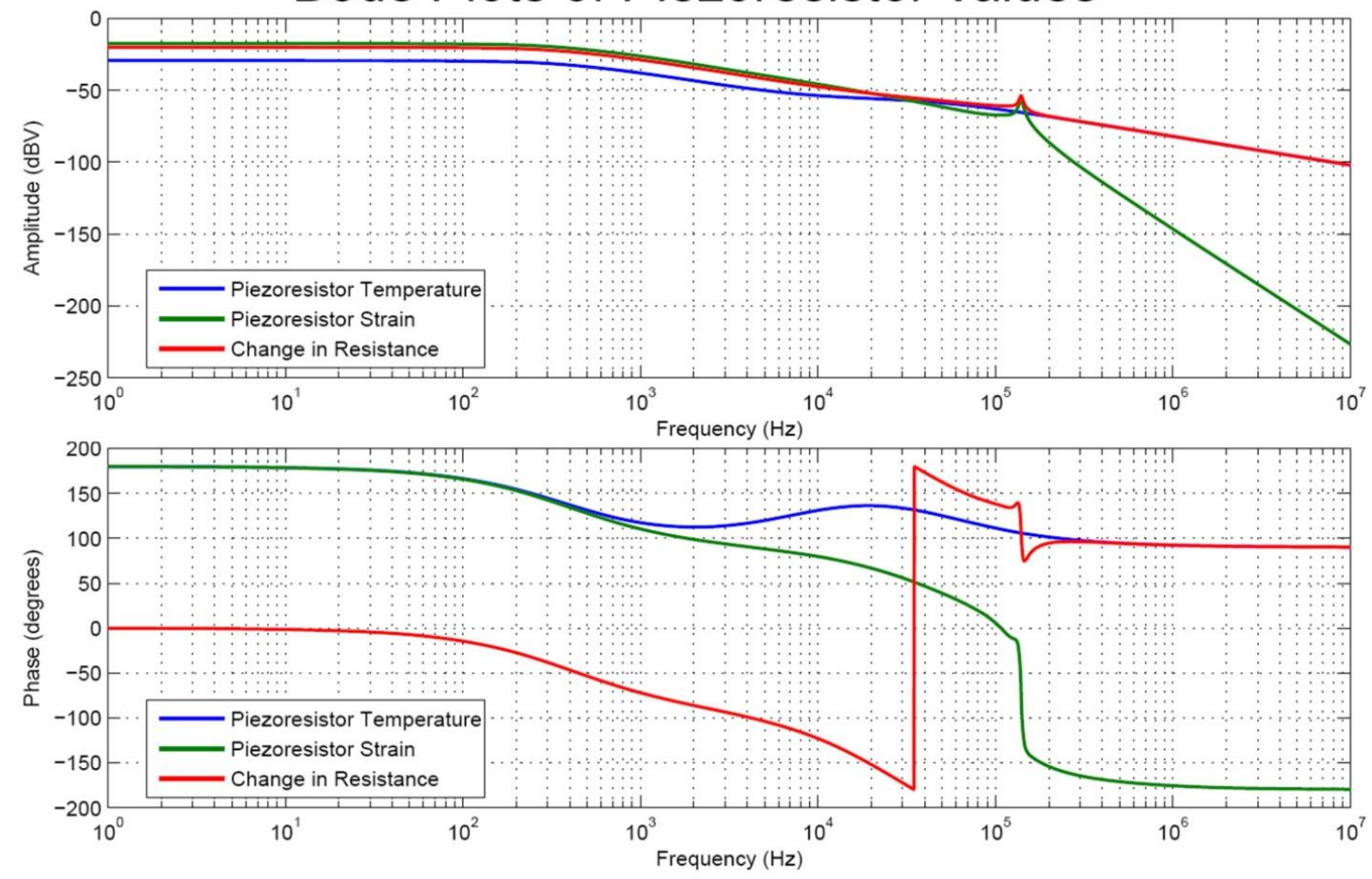

Figure 45: Temperature, strain and resistance change in piezoresistor (simulated)

Bode Plots of Input Signals to Lock-in Amplifier
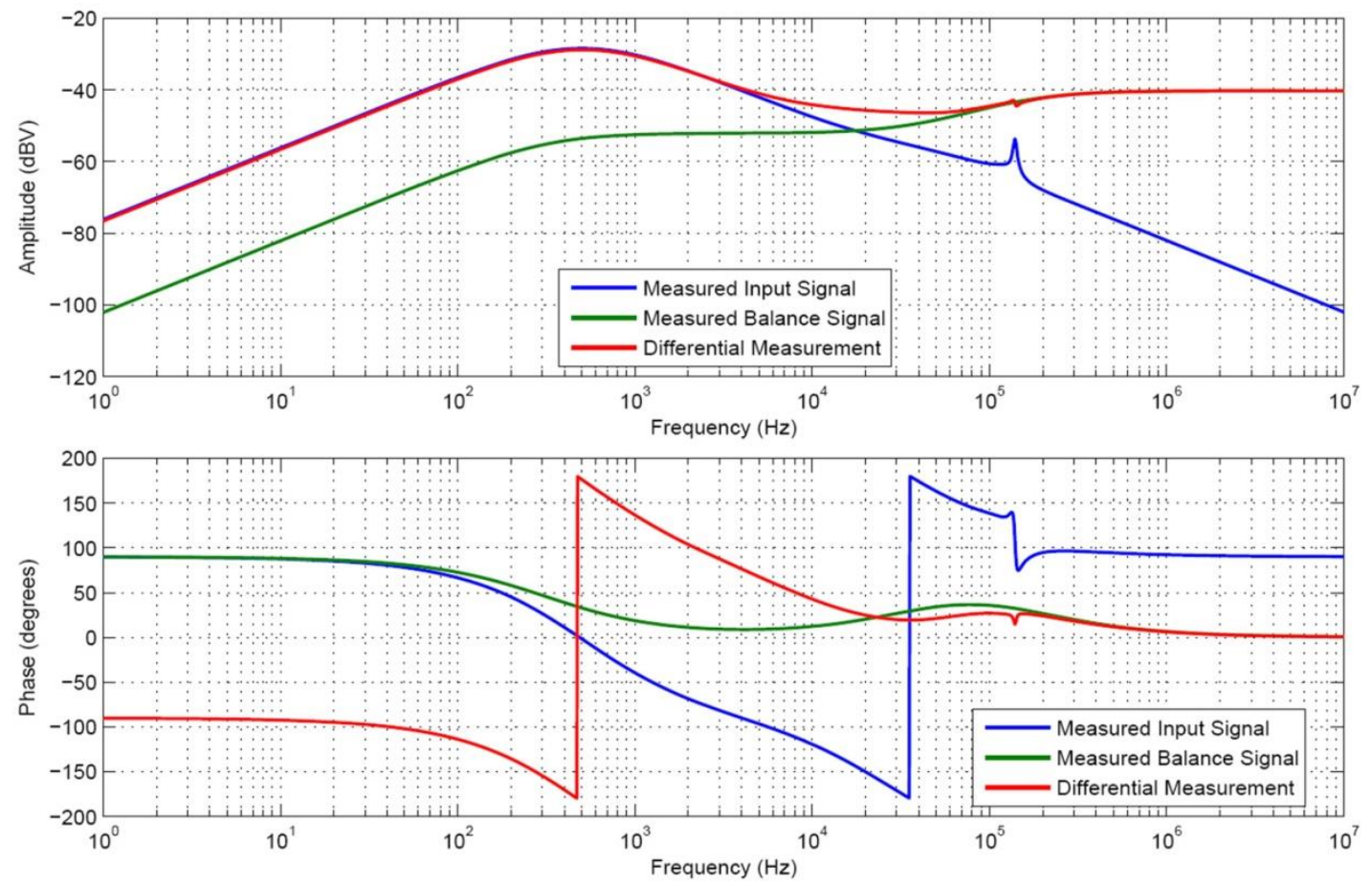

Figure 46: Simulated Bode plots of sensor input, balance input, and differential measurement. 
confined to the balance branch for convenience, where it contributes a zero. Since the measurement setup is differential, only the difference in parasitic coupling is considered. The simulations presented above convey that even with a differential measurement setup, the signal of interest at mechanical resonance is minute in comparison to other effects in the system. Measurements of the quarter-bridge circuit are compared with simulation results in Figure 47. To obtain this data, the bridge was first balanced using an off-chip matching resistor and a potentiometer. With an AC voltage of $50 \mathrm{mV}$ applied, the $\mathrm{DC}$ bias was tuned to $700 \mathrm{mV}, 1400 \mathrm{mV}$, and $2100 \mathrm{mV}$ to obtain the curves shown below. To simulate the effect of a DC bias, the thermal conductivity was tuned.

\section{Bode Plots of DC Biasing Measurements}
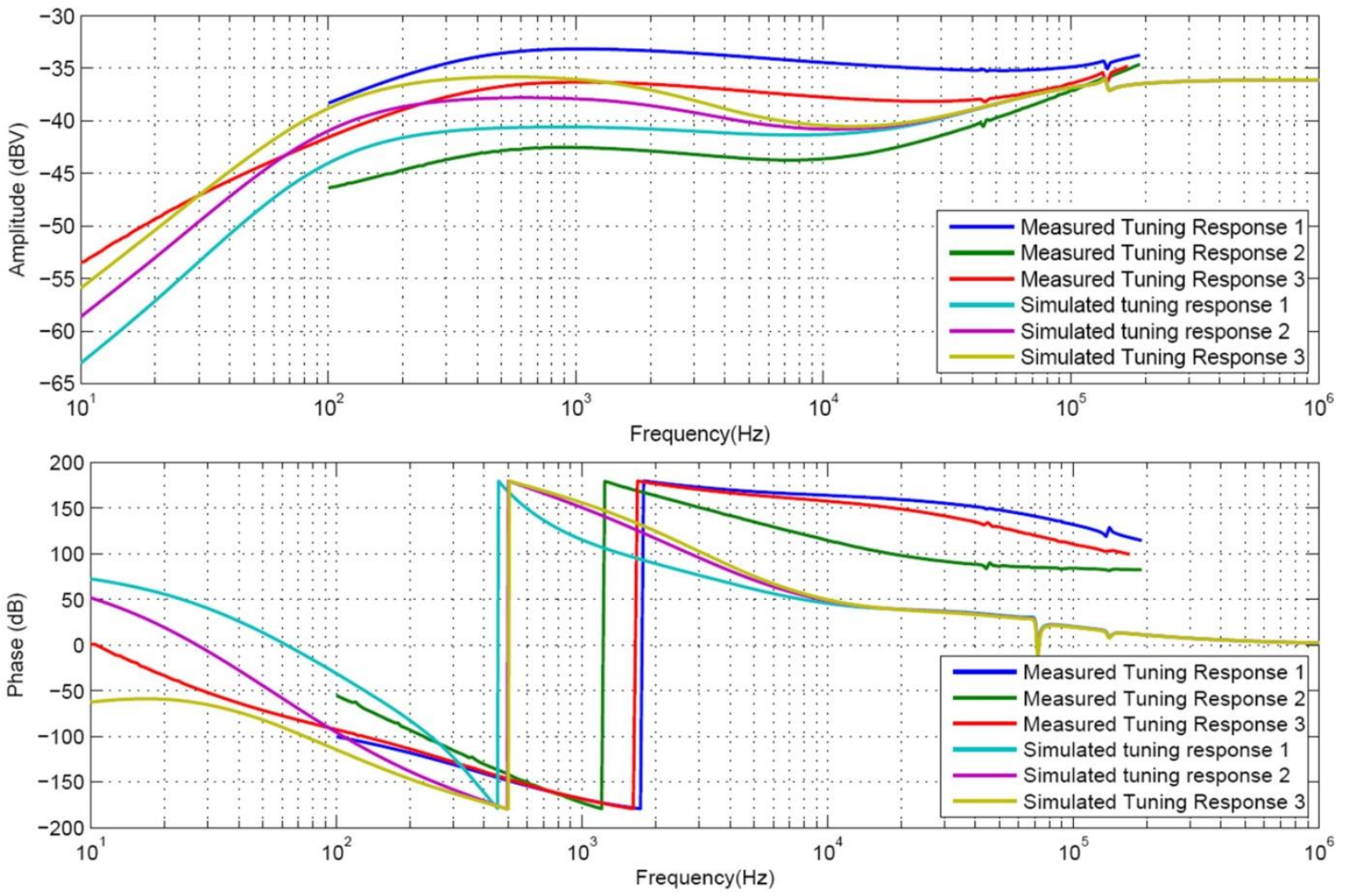

Figure 47: Comparison of simulations to data obtained by tuning the DC bias applied to the bridge

Remarkably, the transfer function of the system changes significantly with the DC bias. This happens in part because of the temperature dependence of the thermal conductivity of polysilicon [174], which adjusts the phase of the signal in order to reshape the transfer function. This property in Silicon has been used to fabricate temperature sensors based on the thermal diffusivity modulation [175]. The 
simulated response with thermal conductivity as a parameter reveals similar tuning behaviour. Tuning the DC bias in the simulation does not alter the response, since none of the linear elements include a DC bias dependence. The precise location of the poles and zeros depends on the values of the input impedance of the lock-in amplifier, the value of the parasitic capacitance, and temperature-dependent material properties of the sample under test. The unknown values are partially responsible for the discrepancy between the measurement and simulations. In addition, these simulations do not account for feedback from the thermal and mechanical domains to the electrical domain. The feedback occurs due to the TCR and piezoresistivity of the material. A third discrepancy arises from the nonlinearity that is caused by the dependence of the electrical resistance on the input voltage. Despite the imperfect match, these results provide an intuitive framework to understand the mechanism behind the electromechanical Q-factor enhancement that may be obtained with CMOS-MEMS thermalpiezoresistive cantilevers in the hybrid mode.

Based on the data presented above, it is clear that the piezoresistive cantilevers must be matched both in amplitude and phase in order to obtain a measurement with reasonable dynamic range. A balance resistor with the same dimensions is thus placed in an arrangement that is thermally symmetric with respect to the sensor, but it does not have a proof-mass attached. The proof mass lowers the thermal pole of the sense resistor, resulting in a relative phase shift that may approach 90 degrees if the poles are far enough apart. This phase shift forms the basis for electromechanical Q-enhancement. At the mechanical resonant frequency, the phase of the piezoresistor signal undergoes a reversal (180 degrees), and crosses that of the balance resistor. If the thermal lag is 90 degrees at this frequency, it is possible to achieve an excellent match by tuning the magnitude with only the real impedances that are available in the potentiometer. Once the bridge is balanced, the phase may be adjusted with a DC bias voltage as demonstrated in the quarter-bridge configuration above.

This type of Q-factor enhancement was observed in several devices, including AM and FM devices with integrated scanners. The data in Figure 48 below was taken with the FM-AFM device that will be discussed in Section 7.1. The DC-bias dependence of $Q$ is evident in the amplitude and phase responses. Although the perceived $Q$ extrapolated from the $-3 d B$ bandwidth and the slope of the phase response indicates a high Q-value, it should be noted that this corresponds only to the electromechanical $Q$ of the transfer function. The benefit of this mechanism is therefore to improve the dynamic range of the measurement of thermal-piezoresistive cantilevers. A similar matching effect is exploited in a Wien Bridge oscillator. 

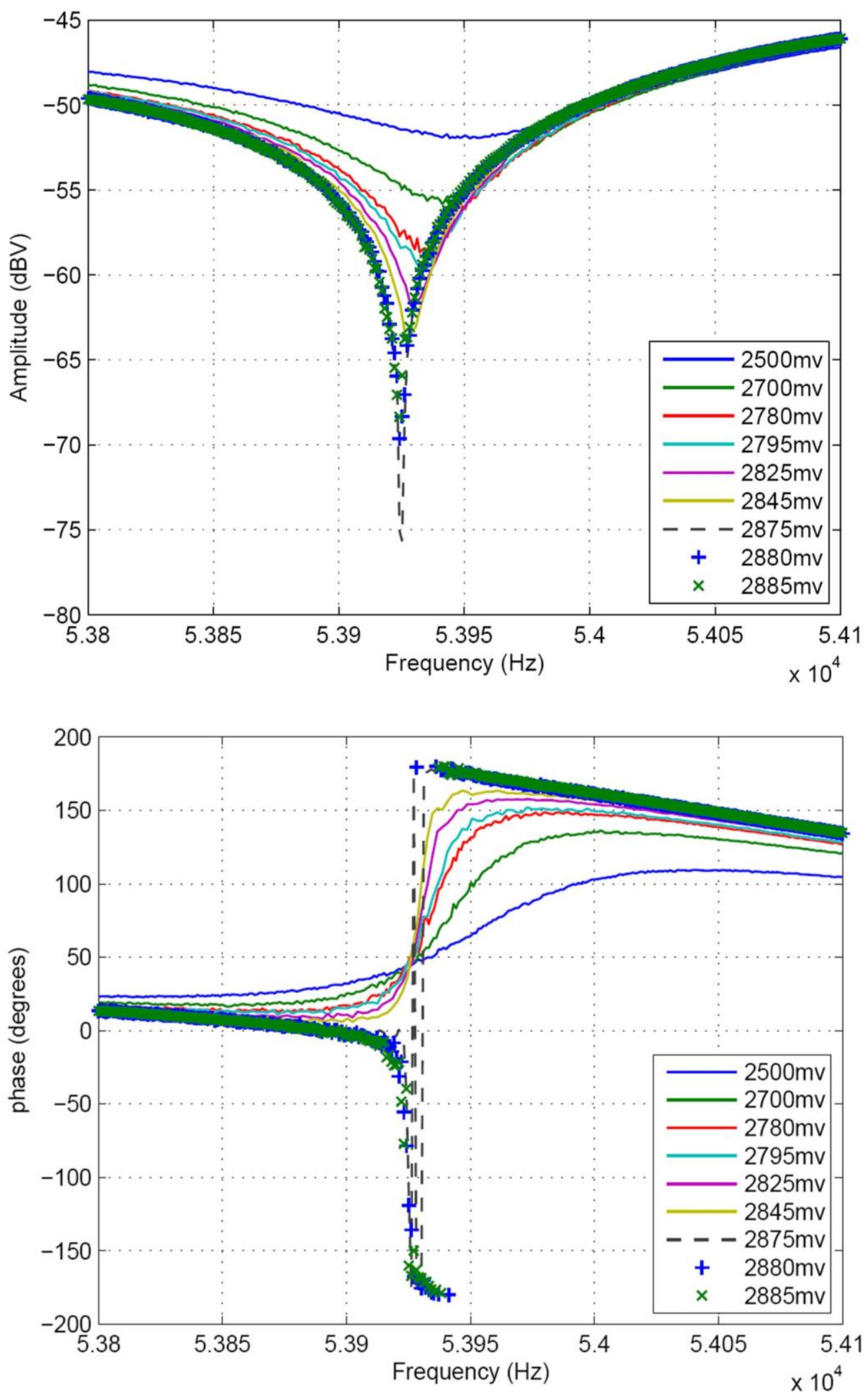

Figure 48: Quality Factor Enhancement as a Function of DC Bias Voltage in a Self-Actuated Resonant Cantilever 
In the context of AFM, it is not necessary for the match to occur at the resonant frequency. In fact, depending on whether the instrument is operating in the repulsive or attractive regime, the preferred frequency of operation is slightly above or below resonance, where the slope of the mechanical transfer function is highest. Under these conditions, the devices in this bridge configuration can provide an excellent match and improve the overall system performance. Figure 48 shows measured results of such a match obtained with a device that has a proof-mass attached. The proof mass lowers the thermal pole of the bimorph as well as its mechanical resonant frequency.

\subsubsection{Mechanical Domain Measurements}

The mechanical Q-factor of these cantilevers sets the minimum detectable force that can be achieved. This fundamental limitation has led researchers to investigate various methods to augment the $Q$ including the use of tuning forks, efforts to reduce anchor losses, operation in vacuum and at low temperatures, selection of low mechanical loss cantilever materials, the use of parametric pumping and negative damping, etc. For AM-AFM, the increased $Q$ reduces the bandwidth of the sensors due to longer settling times from ringing effects. This is not a limitation in FM-AFM, where Q's exceeding 10,000 in vacuum are often used.

To measure the mechanical $Q$ of the cantilevers used in this work, a laser vibrometer was used. The instrument separates the mechanical measurement from artifacts that are present in the electrical measurement. It is shown that the mechanical $Q$ can be adjusted by a factor of about $30 \%$, by tuning the DC bias applied to the piezoresistor at resonance.

Several measurements were performed to quantify the Q-factor of resonant cantilevers exclusively in the mechanical domain. To interpret the measurement results, consider the case of a SARC with negative piezoresistance and negative TCR, operating under a constant DC voltage bias with a small sinusoidal AC signal at the natural frequency of the cantilever beam. The power in the piezoresistor is:

$$
P(t)=\frac{\left(V_{D C}+V_{A C} \sin (\omega t)\right)^{2}}{R}=\frac{1}{R}\left(V_{D C}{ }^{2}+\frac{V_{A C}{ }^{2}}{2}+2 V_{D C} V_{A C} \sin (\omega t)-\frac{V_{A C}{ }^{2} \cos (2 \omega t)}{2}\right)
$$

The temperature therefore contains a large AC component at the fundamental frequency $\omega$, and a small, phase-shifted (quadrature) component at $2 \omega$. It is possible that this second harmonic component contributes to parametric amplification, even though the $2 \omega$ component of the signal is orders of magnitude smaller than the driving force at $\omega$. However, it should be noted that thermal bimorphs with 
stress-free resistors do not show quality factor enhancement. In fact, stress-free bimorph devices suffer from low $Q$-factors of $\sim 50$ that cannot be tuned. This observation suggests that merely including a $2 \omega$ term in the excitation of the resonator is not a sufficient condition to achieve Q-factor enhancement.

Since the resistor is placed in a bimorph, piezoresistive and thermal effects modulate the resistance as a function of the input power, giving rise to nonlinearity. In addition, there is feedback from both domains to the electrical domain, also arising from the TCR and piezoresistivity of the material. The DC bias can be thought of as a reservoir from which power is drawn dynamically by the AC modulation of resistance. This phenomenon may be referred to as a "dynamic power pump". If the pump power is 180 degrees out of phase with the motion of the device, it acts to suppress the temperature change in the actuator. This effect may be modeled as negative feedback, as discussed in Section 3.2.3. If, on the other hand, the resistance variation is in phase, (e.g. if a negative TCR effect dominates and there is no phase lag between the applied voltage and the temperature), the power modulation results in positive feedback. Conversely, if the resistor is driven with a DC bias current, the phase dependence of power is reversed (positive/in-phase TCR results in positive feedback, negative/180-degree TCR results in negative feedback). The diagram in Figure 49 represents the feedback paths and nonlinearity that results from the variation in the resistance, which modifies the electrical domain transfer function. Linear time-invariant methods may not provide an adequate model for such a system.

\section{Thermal-piezoresistive Cantilever System Model}

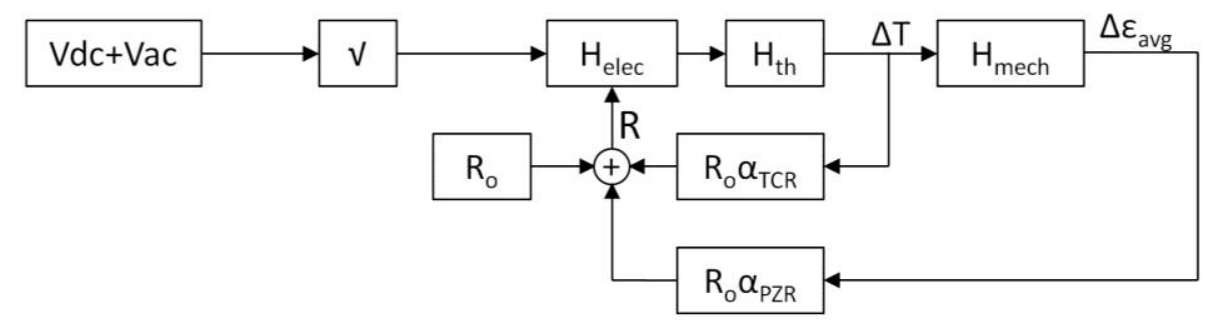

Figure 49: System representation of a thermal-piezoresistive cantilever

It is instructive to examine the frequency-dependent behaviour of this feedback mechanism. At low frequencies (below the $-3 \mathrm{~dB}$ frequency of the bimorph), the resistance and input voltage are in-phase, due to the combination of compressive stress from actuation and a negative coefficient of piezoresistivity that dominates over TCR effects. As the DC bias voltage is increased, the AC resistance 
suppresses the power that is required for periodic Joule heating (more negative feedback). With a DC bias current, power is added in phase with the actuation signal (positive feedback). Thus, the feedback mechanism may influence the amplitude of the actuator's displacement.

One decade above the bimorph's thermal pole (but below the piezoresistor's thermal pole), there is a $\pi / 2$ phase shift between the applied voltage and the temperature and position of the bimorph. No phase shift occurs between the input signal and the piezoresistor temperature. This effectively separates the TCR and strain-dependent contributions to the resistance. When the bimorph is at its maximum deflection, the piezoresistor experiences compressive strain, resulting in an increase in resistance. Since there is a constant DC bias voltage, the power in the resistor is reduced. The temperature of the piezoresistor is reduced instantly, but the bimorph temperature is subjected to a 90 degree phase lag. The opposite occurs when the cantilever is at its minimum deflection - the piezoresistor temperature increases, followed by an increase in the bimorph temperature. In this intermediate frequency range, the thermal pump power provides positive feedback, while the piezoresistive pump influences damping. The range of frequencies for which this occurs may be tailored by sizing the piezoresistor and bimorph in layout. A small resistor and a large thermal mass will extend the frequency range and vice versa.

Above the thermal pole of the piezoresistor, both contributions experience a 90 degree phase shift; however, there is another additional 90-degree phase shift between the generation of piezoresistive pump power and bimorph temperature, arising from the lag in the thermal response of the piezoresistor. The 180 degree phase shift between the piezoresistive pump power and the bimorph position means that the strain effect provides positive feedback, while the TCR influences damping. At high frequencies, the attenuated amplitude of thermal fluctuations and mechanical motion suppresses the pump power.

In each frequency regime discussed above, the designer may also wish to incorporate a mechanical resonance. Mechanical resonance adds 90 degrees of phase lag to the motion of the cantilever while leaving the phase of the TCR effect unchanged. At low frequencies, the TCR pump power continues to provide positive or negative feedback (depending on the choice of voltage or current source) while the resonant piezoresistive pump influences damping. If the resonance occurs in the intermediate frequency range, the TCR and piezoresistive modulation both provide feedback, but the sign of the piezoresistive pump is reversed to achieve a net positive feedback. Finally, at high frequencies, both 
pump contributions influence the damping. However, because of the reduced amplitude of the temperature and position fluctuations, compensation of the damping term is reduced.

The preceding discussion considers the contributions of the each pump individually on the basis of its phase in a given frequency regime. In reality, the phase and amplitude of the transfer function for the total pump power has a continuous variation in phase and amplitude, caused by the difference in the TCR and piezoresistance coefficients as well as the relative magnitudes and phases of temperature and position at a given frequency. The regions in which Q-enhancement may occur must therefore be determined by examining the overall frequency response of the device, which was explored in Figure 45.

It can now be deduced that many combinations of feedback and damping may be achieved by tailoring the frequency response of a thermal-piezoresistive cantilever. One example of a scenario in which the piezoresistive pump may compensate the damping term at resonance is depicted below in Figure 50.
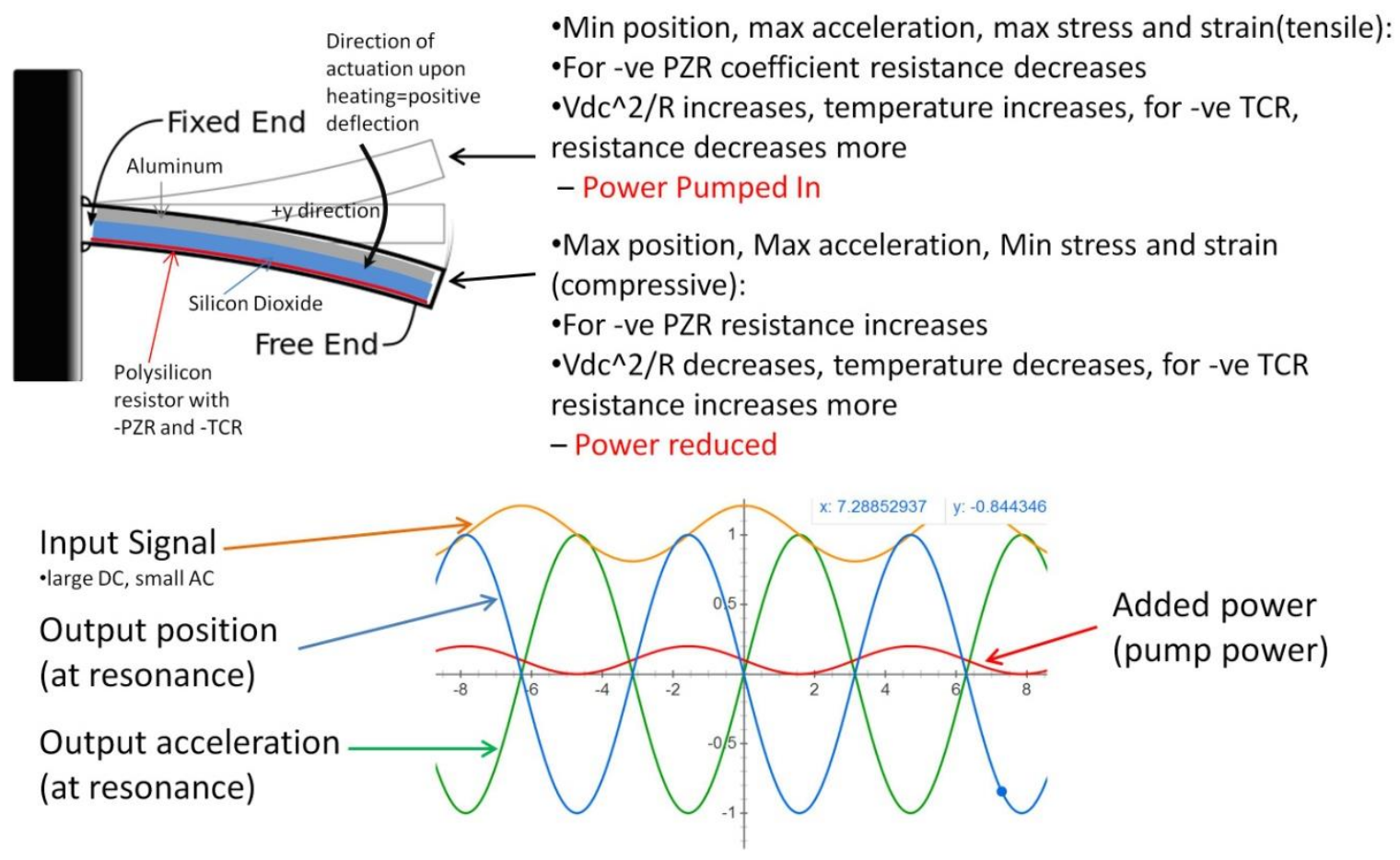

Figure 50: Quality Factor Enhancement Under Driving Force Control

It is interesting to note that the distribution of strain in the piezoresistor is perfectly matched to its mode shape in resonance, so that the pump power is also proportional to the deflection at any point in the beam. This mode-matched pumping may enable higher order modes to be enhanced, since regions 
with large positive curvature will experience less pumping while regions with large negative curvatures will receive more power.

The efficiency of this mechanism depends on the heat capacity and thermal conductivity of the beam. If the piezoresistor is integrated throughout the length of the beam, the time constant associated with heat propagation is only a function of the thickness of the beam. This is in stark contrast to the base or tip heating methods used for vertical actuation, where the entire volume of the cantilever contributes to the thermal mass that loads the heater. Nevertheless, the thickness of the beam imposes an upper limit on the frequency at which this pumping mechanism is efficient. Beyond the vertical thermal time constant of the beam the thermal wave constricts and experiences a phase shift that may be modeled with a transmission line in the vertical direction. At low frequencies, the lateral (i.e. axial) propagation of heat will "smear out" the temperature distribution, an effect that is deleterious to the localized mode-shape matching.

To quantify the mechanical $Q$, several experiments were performed on thermal-piezoresistive cantilevers. The step response was first captured to observe ringing at the natural frequency of the beam (Figure 51). This measurement was repeated at various DC bias levels in order to determine whether the pump power contributes to the $\mathrm{Q}$-factor. The rising edge step response data reveals the $\mathrm{Q}$ factor with an applied DC bias, while the falling edge data measures the response to a step input with no DC bias. The Q-factor was extracted by fitting an exponential curve to the envelope of the ringing response. In order to remove the effect of the RC time constant in the thermal domain, the average displacement of the cantilever was first subtracted from the data, yielding a symmetric envelope.

\section{Quality Factor from Step Response Measurements}
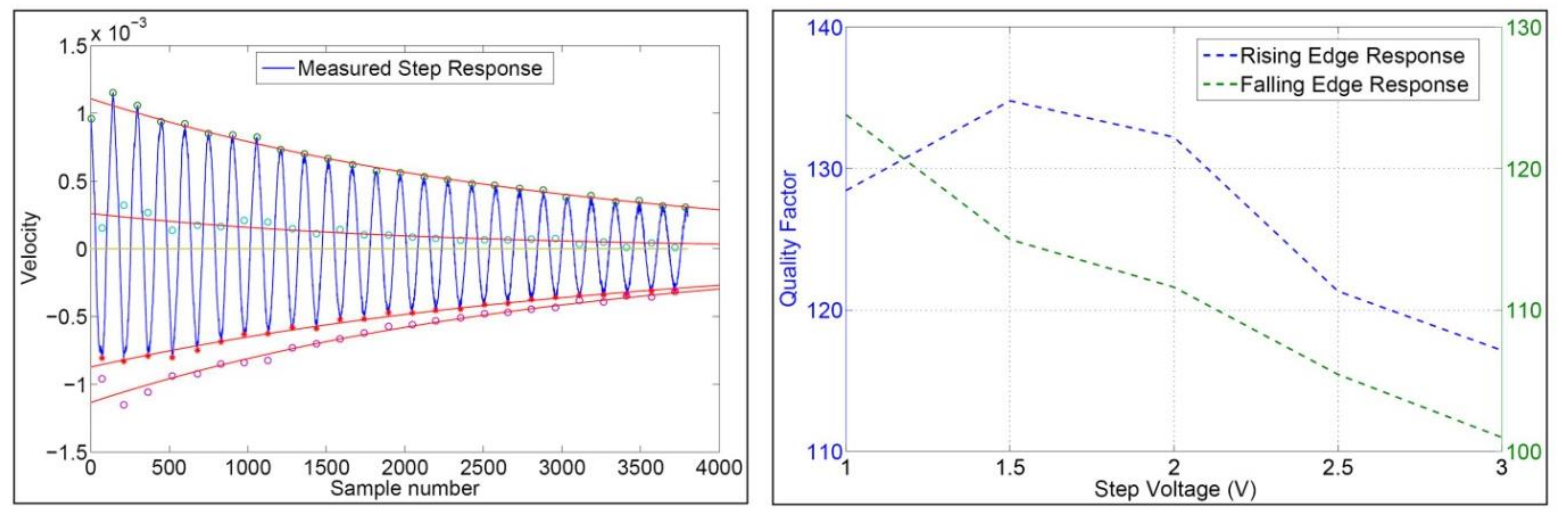

Figure 51: Quality Factor Extracted from Ringing Envelope 
The data shows that a DC bias voltage increases the Q-factor of this device, which has a resonant frequency of $\sim 5.5 \mathrm{kHz}$. The $\mathrm{Q}$ enhancement reaches a maximum at $1.5 \mathrm{~V}$, and then falls off. In all cases, the $Q$ with a $D C$ bias is higher than the $Q$ without a $D C$ bias, indicating that energy from the $D C$ bias is periodically pumped into the system. Since there is no periodic excitation in this measurement, the pump power can only arise from piezoresistive modulation, and not from the TCR. Interestingly, the temperature dependence of the Q-factor is negative, as can be inferred from the 0-DC bias measurements (falling edge) which are taken before the device has reached thermal equilibrium.

In the next set of measurements, a small AC input signal was applied in combination with a DC bias. At the instant that the AC component was turned off, the ring-down envelope was captured and fitted. Data was obtained using both current and voltage sources, with varying DC bias levels. The measured results (Figure 52 ) indicate a resonant frequency of $\sim 140 \mathrm{kHz}$.

\section{Quality Factor from Ring-down with DC Bias}
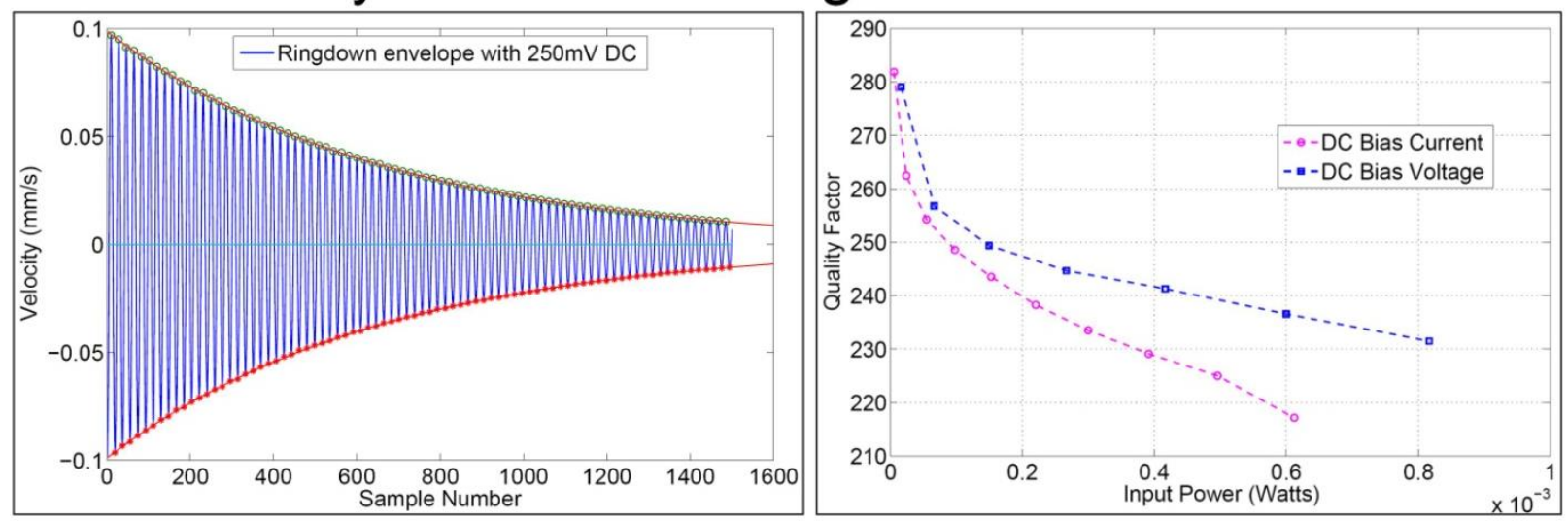

Figure 52: Left, extraction of envelope after removal of AC signal. Right, Q-factor as a function of DC bias power

Only the piezoresistive pump's contribution is present in this data, since the measurement is performed after the $A C$ signal is removed. The downward trend in both curves shows that the temperature coefficient of the Q-factor is negative, as with the previous device. Since there is a 180 degree phase difference between the pump powers in the two cases, it may be assumed that current drive further increases the damping, while voltage drive reduces it.

Another set of measurements was performed on this device in order to ascertain the TCR contribution to the energy pumping mechanism. In this case, the response was measured when the AC component was turned on, and the resulting envelope is shown in Figure 53. As the input power is increased, both 
the current and voltage responses show improved Q-factors up to a maximum value of $\sim 240$, followed by a reduction in $\mathrm{Q}$. In both cases, the effect of including the TCR contribution is to lower the Quality factor of the resonator when compared to results obtained with only piezoresistive pumping. These results suggest that as the phase of the pump is adjusted by the DC-bias power, a threshold is crossed at which one pumping mechanism begins to dominate over the other.

\section{Quality Factor from Ring-up}
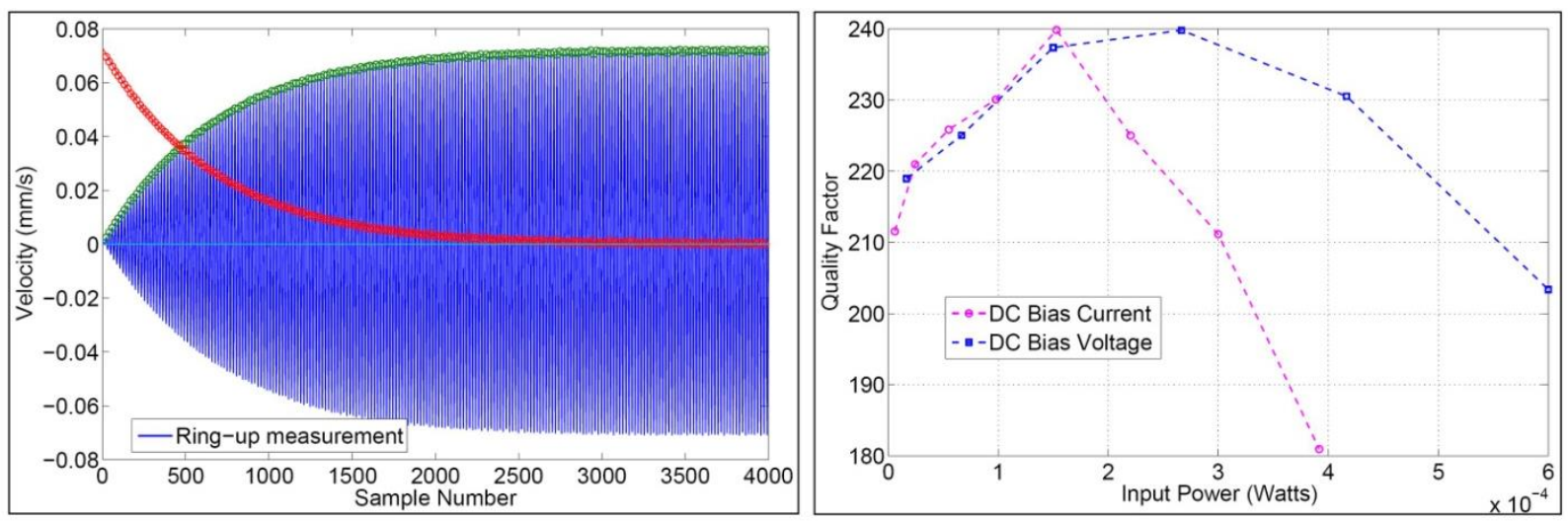

Figure 53: Left, extraction of envelope upon addition of AC signal. Right, Q-factor as a function of DC bias power.

Laser vibrometer measurements were also performed to quantify the nonlinear effects that occur in this system. When the pump power exceeds a threshold level, higher harmonics can be observed in the frequency response of the cantilever. At low frequencies, negative feedback from piezoresistivity suppresses the amplitude of the cantilever response. Near the thermal roll-off frequency, the amplitude of the cantilevers motion is sufficient to modulate its resistance due to both TCR and strain effects. Since the thermal pump does not experience a phase shift, it contributes positive feedback while contribution of the piezoresistive pump is reduced and phase-shifted. At these frequencies, a large number of higher harmonics are clearly observable as shown in Figure 54. At intermediate frequencies, the threshold of power required to observe higher harmonics increases until the required power exceeds the power-handling capability of the device. This is expected, since the thermal pole relating to the bimorph temperature contributes a roll-off in the amplitude response of the cantilever, even though the temperature fluctuations may remain at the same magnitude.

Interestingly, there are isolated points in the intermediate frequency regime (above thermal roll-off) that do generate a large number of harmonic peaks. These frequencies are integer fractions of the 
natural frequency of the cantilever, suggesting that the piezoresistive pump is engaged. When the excitation frequency coincides with an integer sub-multiple of the resonant frequency, harmonics appear above and below the resonance, as shown in Figure 55. When the excitation frequency is $1 / 4$ of the resonant frequency, 19 peaks are observed in the frequency spectrum of the device.

\section{Excitation of Harmonics Above Roll-off}

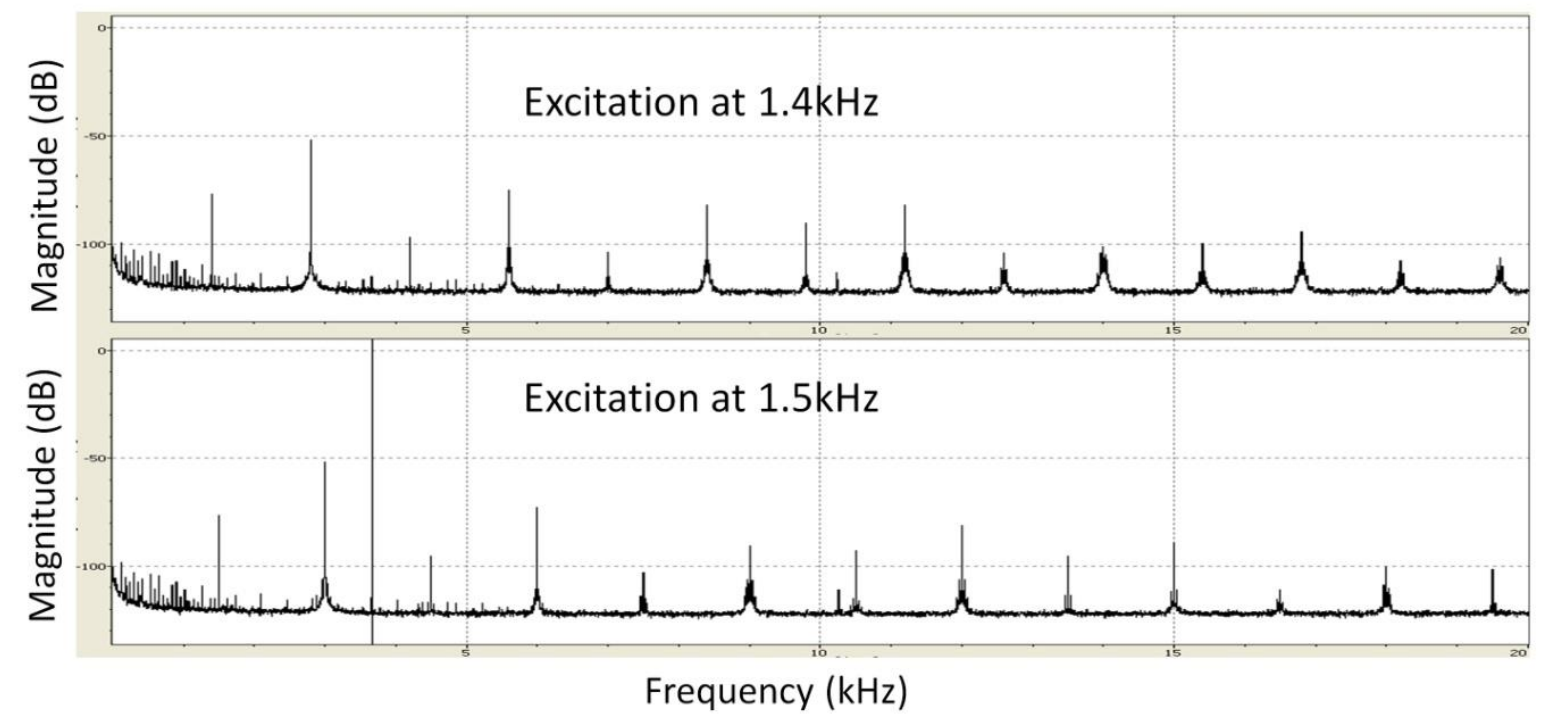

Figure 54: Low frequency excitation at $1.4 \mathrm{kHz}$ and $1.5 \mathrm{kHz}$

Superharmonic Resonance

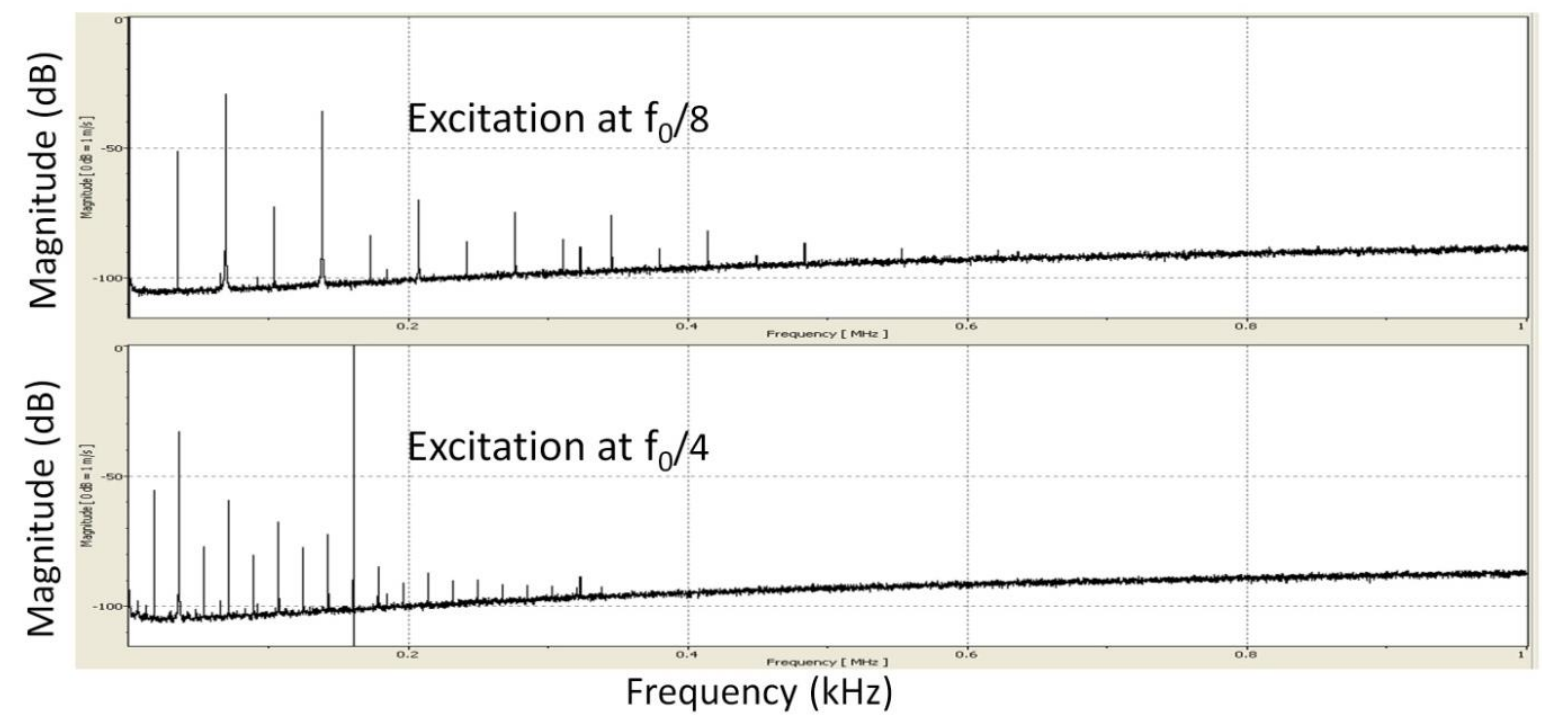

Figure 55: Superharmonic excitation of resonance in thermal-piezoresistive cantilevers 
The generation of higher harmonics at intermediate frequencies is most likely triggered by thermal effects, because the strain in the bimorph is reduced after the $-3 \mathrm{~dB}$ point. Recall that the temperature of a self-heated resistor under an applied voltage may be represented by the following differential equation, before linearization:

$$
\frac{d T}{d t}=-\frac{T}{R_{t h} C_{t h}}+\left(\frac{\left(V_{D C}+V_{A C}\right)^{2}}{R_{0} C_{t h}\left(1+\alpha_{R} T\right)}\right)
$$

This is a first order nonlinear differential equation which can be solved numerically. Figure 56 is a plot that reveals the behaviour of the system with a sinusoidal AC input and a DC offset. At sufficiently high DC bias, the gain from self-heating becomes apparent. In the simulation, the value of $\alpha_{R}$ was varied as a parameter.

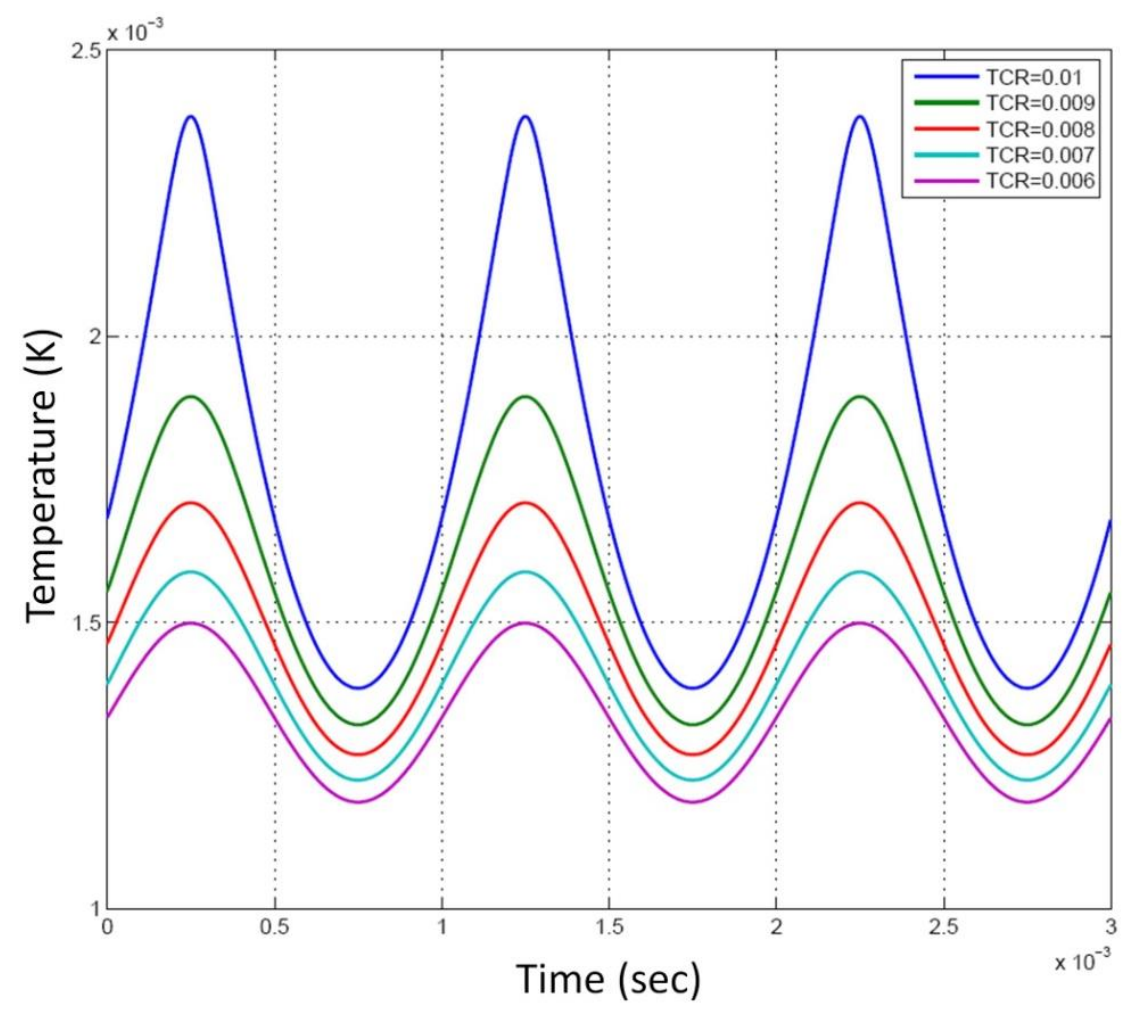

Figure 56: Thermal Runaway in the SARC Model. Time domain representation of the temperature in a resistor experiencing self-heating, with various values for the TCR.

For the purpose of incorporating these effects into a practical lumped element model that reflects the operating conditions of the SARC, one may assume a small TCR $\alpha_{R}$ and ignore the AC contribution due to Joule heating from the small AC signal. The time constant may be calculated as was done in equation (3.4): 


$$
\tau_{V}=\frac{R_{t h} C_{t h}}{\left(1-\frac{\alpha_{R} R_{t h} V^{2}}{R_{0}}\right)}
$$

Where the minus sign in the denominator has been inserted to point out that $\alpha_{R}$ is negative. The time constant of this system may be modulated by the DC bias, implying that the phase of the pump-power may be adjusted to an extent in this manner.

The steady state temperature response of the system may be used to calculate the instantaneous temperature surrounding the resistor:

$$
T=\frac{\frac{R_{t h} V^{2}}{R_{0}}}{\left(1-\frac{\alpha_{R} R_{t h} V^{2}}{R_{0}}\right)}
$$

This expression may be recognized as a linear system with positive feedback, with a gain term that is proportional to $\alpha_{R}$. A similar analysis may be carried out for the piezoresistive pump, and would reveal that piezoresistance may also contribute positive feedback if a frequency point is chosen with the correct phase and amplitude. In this scenario, piezoresistivity triggers a temperature change that propagates to the bimorph in phase with the actuators position. These mechanisms may give rise to higher harmonic terms and contribute parametric effects and possibly Q-enhancement.

Although previously reported thermal piezoresistive pumps required deliberate processing to optimize the piezoresistivity, this method makes use of a standard CMOS polysilicon material with moderate strain sensitivity. This can be attributed in part to the gain in strain that is attained by offsetting the placement of the sensor with respect to the neutral axis of the beam. Also, the small signal from strain may be amplified by the positive feedback from the TCR effect.

To summarize, a novel $Q$ enhancement mechanism has been implemented in self-actuated resonant cantilevers. Electromechanical $Q$ improvements of $>1000 x$ have been obtained. It is believed that several frequency-dependent mechanisms contribute to the measured results. In the electrical domain, reactive matching of the Wheatstone bridge provides a sharp null near the resonant frequency, when the phase and amplitude of each half of the bridge are matched. This improves the dynamic range of the measurement setup, but does not enhance the minimum detectable force of the cantilever. Measurements in the mechanical domain reveal that piezoresistive and thermal energy pumps may contribute to Q-enhancement, depending on the phase and amplitude of resistance modulation at the 
operating frequency. In addition, measurements of superharmonic excitation have confirmed that parametric effects are present, which may arise from positive feedback in the pumps.

Due to the distributed nature of the resistance modulation, the piezoresistively pumped energy is matched to the mode shape of the resonator. An upper limit to the attainable frequency is set by the vertical thermal time constant of the beams. Thinner bimorphs made with high diffusivity materials (e.g. Aluminum and Tungsten) may be used to increase the operating frequency of the devices, provided that their TCR and gauge factors are large enough. It is believed that the Q-pumping mechanism can be used with other resonator geometries, such as bulk resonators, simply by incorporating a DC current path through a judiciously chosen piezoresistive material. Finally, because the mechanism relies on temperature oscillations, it is a good candidate for SThM. In the SThM context, a high Q mechanical resonance may provide gain to a signal in the thermal domain by placing the base of the resonant flexure (which experiences maximum strain and pump power) in direct thermal contact with the tip of the microscope. Hence, small changes of heat conduction in the tip-sample region may be amplified mechanically to acquire sensitive measurements of local thermal conductivity and thermal capacitance. 


\section{Chapter 5. Drive-Sense Strategies in the Presence of Noise}

The models developed thus far are useful in guiding the design of single-chip SPM's because they capture the interplay between various quantities in the electrical, thermal, and mechanical domains; however, in the real world, the interface to instrumentation exists uniquely in the electrical domain. Whether the tip-sample interaction is thermal, mechanical, or electrical, the driving and sensing schemes to actuate and detect are of great importance. This chapter discusses the electrical strategies that have been adopted to drive actuators, sense position, and detect small signals in the presence of noise.

The methods that have been developed to drive electrothermal actuators include analog drive with a square-root circuit in the feed-forward path, pulse-width-modulation (PWM), sigma-delta drive $(\Sigma \Delta)$, and an 8-bit microelectromechanical digital-to-analog converter (MEMDAC). Closed loop control of the actuators is achieved with the use of integrated temperature sensors, because the largest disturbance to the actuators arises from thermal coupling. The fundamental noise limits of the drive-sense strategies are discussed, and a modulation technique is implemented to mitigate the deleterious effects of $1 / f$ noise. The same limitations and strategies exist for resistive temperature sensors that are used in SThM.

The methods used to detect forces through piezoresistive strain measurements are also discussed in this Chapter. For static piezoresistive sensors, various sources of noise set the minimum detectable force. Dynamic piezoresistive sensing is subject to the same noise constraints, but the Q-factor of a resonant sensor may enhance the signal-to-noise ratio. The TCR of the polysilicon material used in these devices generates a large disturbance in response to tip-sample heat conduction and thermal coupling from actuation. Careful matching must be performed in the design process to reject the thermal component of the signal.

\subsection{Linearization}

The voltage to position transfer function of an electrothermal actuator contains several nonlinearities. The thermal moment that is generated by Joule heating is proportional to the power dissipated in the heater resistor, giving rise to a square-law dependence. The TCR and piezoresistance of embedded polysilicon also contribute nonlinearities. The deflection of a bimorph also contains a nonlinear term that becomes significant at large displacements, when the assumption of small slope breaks down. Under the assumption of small temperature excursions and small deflections, the dominant issue is the 
square-law dependence. This issue is particularly problematic for vertical electrothermal actuators that are operating with a superposition of static and dynamic inputs.

In dynamic AFM, the vertical actuator may track topology with a DC input, while oscillating at its resonant frequency. The $A C$ input amplitude remains constant, while a DC bias is used to offset the position of the tip according to sample features. As shown in the plot of Figure 41 in section 4.2.6, the vibration amplitude is modulated by the DC input. The problem is most significant while imaging because the when the cantilever experiences a force gradient from tip-sample interaction, the amplitude of the force input influences the natural frequency of the system, and hence it distorts the detected signal. In fact, it is sometimes desirable to modulate the amplitude of the forcing function independent of the offset voltage to quantitatively reveal material properties as discussed in [176].

A square-root circuit is placed in the feed-forward path to mitigate this issue, as was illustrated in Figure 14. The SEM image is a slow scan of a lateral actuator that is deflecting in the plane of the chip. With the square-root circuit in place, the input voltage to output displacement transfer function is linearized. When used in a vertical actuator, the vibration amplitude remains constant while tracking topology.

\subsection{Digital Drive}

PWM is a common method to drive devices with linear power dependence, where the power delivered to the load has a linear dependence the duty cycle. A $\Sigma \Delta$ implementation may also be used to drive these actuators. The term $\Sigma \Delta$ DAC is often used to describe the combination of a digital-to-digital converter which up-samples an n-bit signal at a frequency $f_{s}$ to an m-bit sample at frequency $\frac{n}{m} f_{s}$, then uses an all-digital $\Sigma \Delta$ to reduce the word length, and finally outputs the signal using high speed m-bit resolution DAC. Both of these methods require a filter at their outputs in order to remove quantization noise. The filter usually occupies a significant fraction of the footprint of the device in a CMOS implementation. Electrothermal actuators possess a low-frequency pole in the thermal domain due to their thermal capacitance. The thermal RC circuit may be thought of as a low-pass filter in the transfer function of the actuator, which attenuates the high frequency electrical quantization noise.

PWM and $\Sigma \Delta$ drive schemes were implemented on a field-programmable gate array (FPGA). Using a PWM driver, the resistance of a lateral actuator was measured and is plotted as a function of duty cycle below in Figure 57. The step response of the actuator's resistance was also captured using an input step voltage, a step input to the set-point of an analog PID controller, and step inputs to the set-points of PID controllers with PWM and $\Sigma \Delta$ drive stages (Figure 57). 


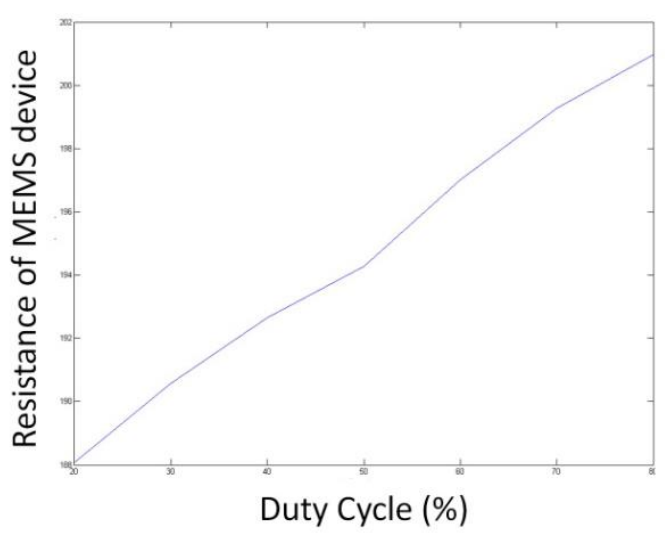

PWM at $20 \mathrm{kHz}, 20-80 \%$ Duty Cycle

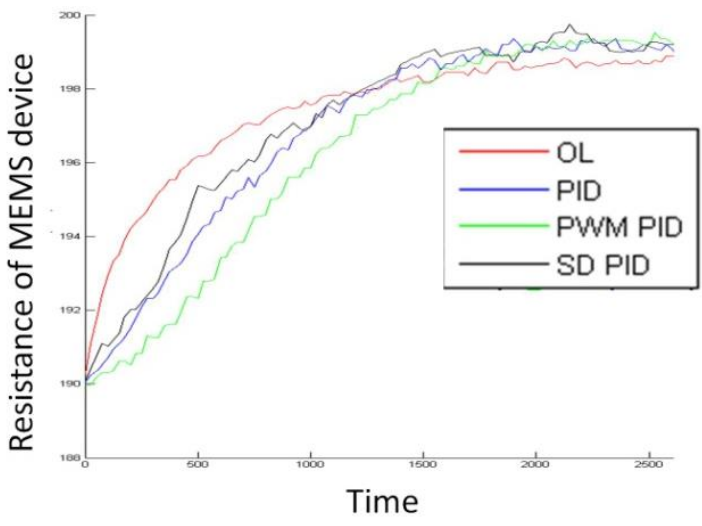

Comparison of Open Loop, Linear PID, PWM PID and SD PID

Figure 57: Left, linear response of electrothermal actuator to a PWM signal. Right, step response obtained in open-loop, with an analog PID controller, with closed-loop PWM, and with closed-loop Sigma-Delta drive.

The sampling frequency of these devices should be chosen well-beyond the thermal cutoff frequency of the actuator. In addition, it should be far-removed from mechanical resonances. The bandwidth of the output signals in these methods is limited by their clock speed and inversely proportional to the desired resolution. Lateral scanning in an SPM requires high position resolution, but is generally not faster than $\sim 100 \mathrm{~Hz}$. Thus, these drive methods are more practical for use with lateral scanners than with vertical actuators that must operate at the natural frequency of a flexural resonator.

\subsubsection{MEMDAC}

Yet another method to control the position of a MEMS device is known as a MEMDAC. The device is directly analogous to an R-2R electronic digital-to-analog converter (DAC), with the resistors replaced by cantilevers with compliances of $\mathrm{C}$ and $2 \mathrm{C}$, as shown in Figure 58. 


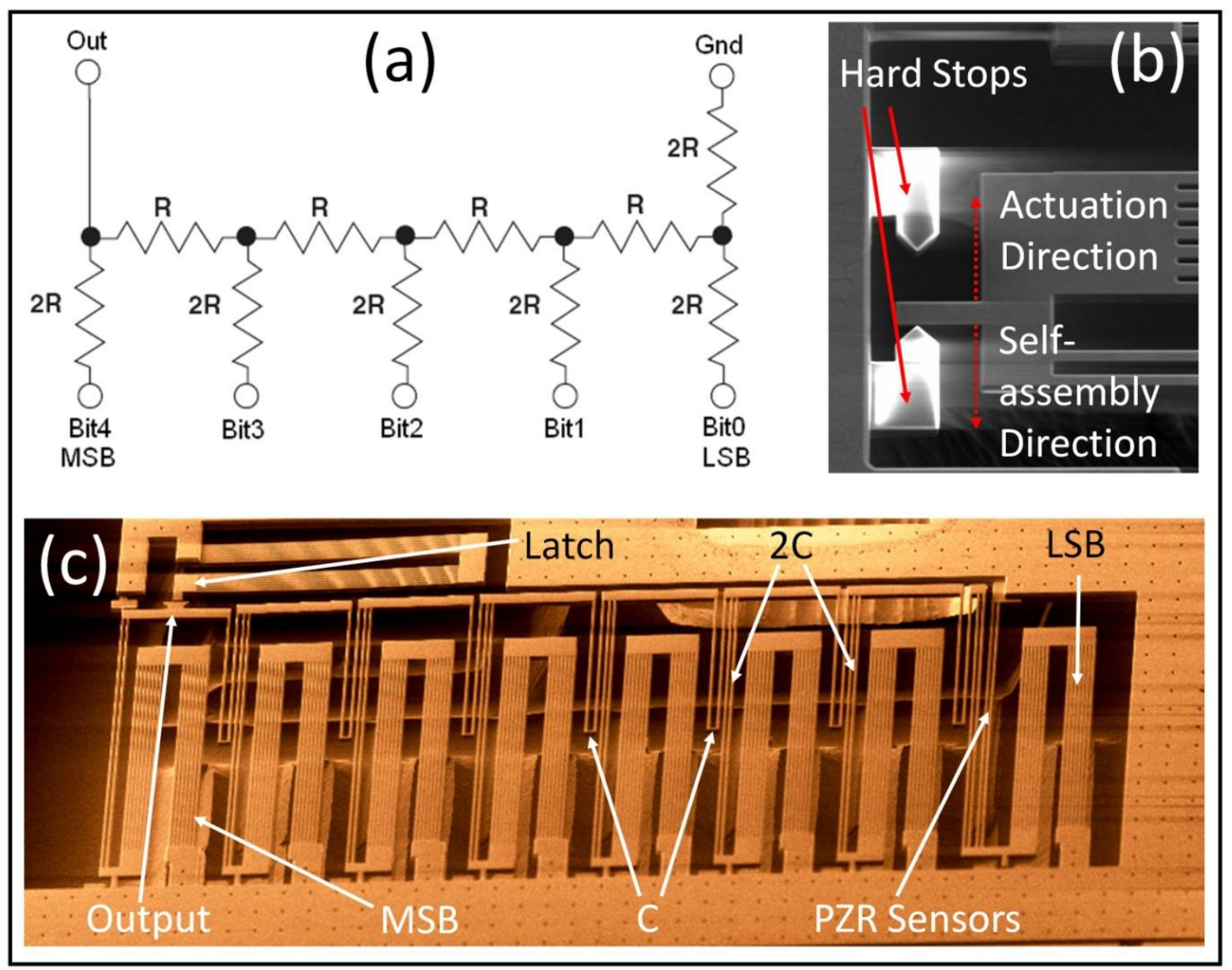

Figure 58: Electronic R-2R DAC (a) and MEMS implementation (c). SEM image shows beams of compliance $\mathrm{C}$ and $2 \mathrm{C}$ in the network. A latch is present for power-off positioning at the output, and the LSB and MSB are indicated. The piezoresistive strain gauge is located within the load beam near the LSB. Hard stops are used to limit the input position to 2 distinct values (b).

An 8-bit MEMDAC based on previously reported architectures [177], [178] was fabricated in the CMOSMEMS process. The design was augmented in the following ways:

1. The number of bits has been increased to 8 in order to achieve a step-size of $31 \mathrm{~nm}$.

2. The LSB contains a piezoresistive position sensor for sub-positioning within a bit. The piezoresistive position sensor has an output-referred resolution of $<1 \mathrm{~nm}$.

3. A CMOS scan chain and current drivers are integrated with the device in order to control multiple MEMDACs with a 5-wire interface.

4. A latch has been placed at the distal end of the device for power-off positioning.

5. A 2-D positioner with an integrated cantilever has been implemented using four 1-D MEMDACs 
The actuators that provide input displacements into the compliance network are limited by hard-stops in order to provide distinct positions of $0 \mu \mathrm{m}$ and $8 \mu \mathrm{m}$. A lateral self-assembly displacement occurs due to the residual stress mismatch in the lateral bimorph materials, so the hard-stops are judiciously shifted to account for this effect. In placing the hard-stops, one must also consider thermal coupling from adjacent actuators through the compliance network, substrate, and ground shield. These sources of heat flow result in partial actuation of a bit that is intended to be "off" when adjacent bits are "on." The hard stops must therefore be placed conservatively so that this partial actuation does not displace the actuator from its "off" position.

An additional actuator is placed orthogonally with respect to the output of the MEMDAC, and serves as a latching element. This actuator self-assembles into the engaged position upon release, eliminating power consumption while the device is off.

The LSB in the MEMDAC connects to a load beam with integrated differential piezoresistive strain sensors. This geometry, shown in Figure 59, is intended to ensure that the resistors are temperaturematched, but they experience opposite stress. The polysilicon must be inset from the outer edges of the beam for protection during the silicon release process. This compromises the sensor resolution, since the piezoresistors are located near the neutral axis of the beam. An off-chip bridge circuit is used to measure position with a resolution of $100 \mathrm{~nm}$. A $100 \mathrm{~nm}$ position resolution in the LSB corresponds to $<0.5 \mathrm{~nm}$ displacement at the output.

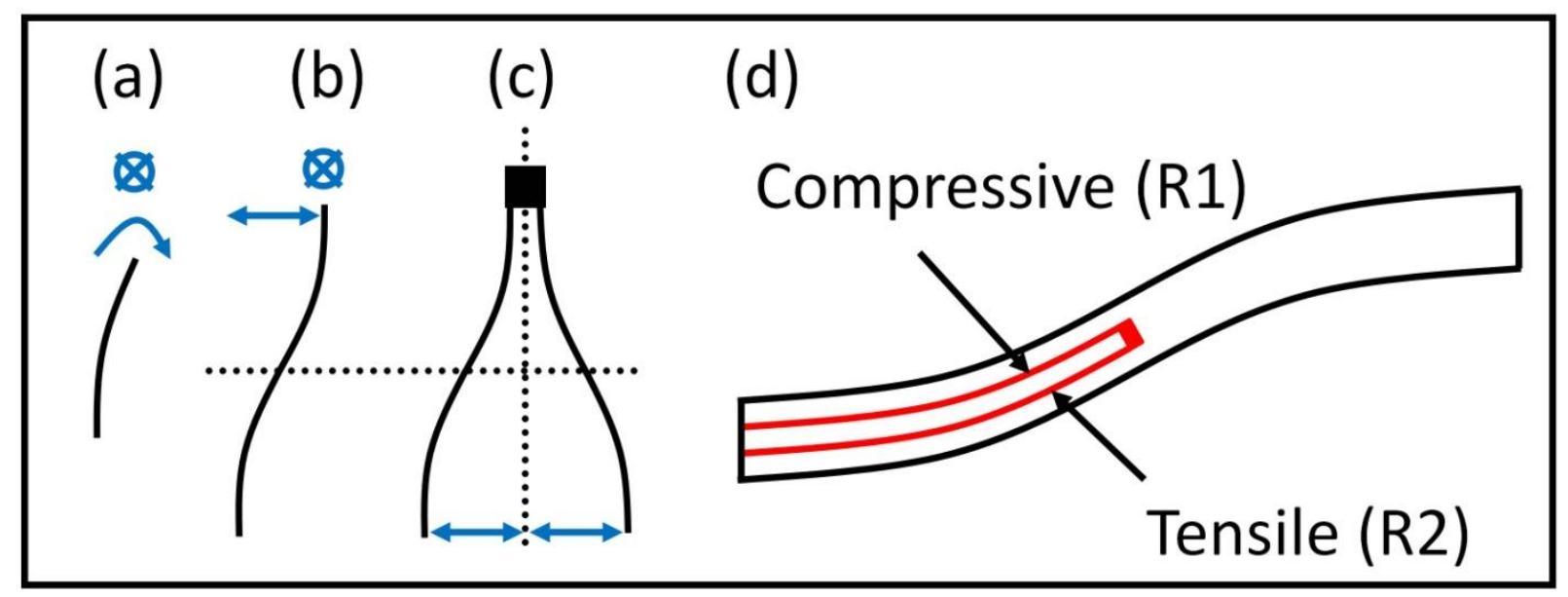

Figure 59: Location of Piezoresistors to Measure Lateral Position in LSB

An equivalent circuit of the mechanical system is shown in Figure 60. The beams are considered to be pure springs and are represented as capacitors with a value of: 


$$
C_{e}=\frac{1}{k_{m}}
$$

Where $C_{e}$ is the capacitance in the electrical model and $k_{m}$ is stiffness of the beams. Because the hard stops fix the position of the beams, a fixed amount of charge is added to each capacitor that is assigned a value of "1." The output voltage is calculated after charge sharing has occurred and corresponds to the binary weighted sum of the digital input. The capacitance looking into the compliance network remains constant at $2 C_{e}$ regardless of the number of bits, so the charge that is available to deflect the load capacitance may be calculated with:

$$
Q_{\text {net }}=C_{M E M D A C} \times V_{\text {out }}=2 C_{e} V_{\text {out }}
$$

If a load compliance is placed in parallel with the MEMDAC, this charge is shared with the load, scaling the output voltage.
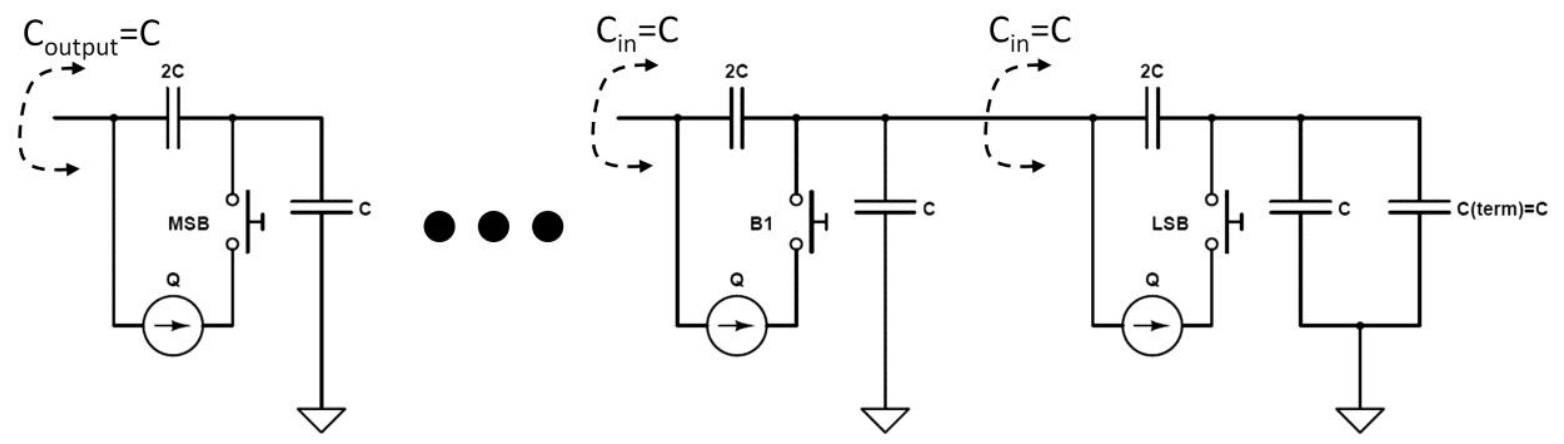

Figure 60: Impedance Looking Into the Compliance Network

The MEMDAC's compliance network provides a geometric advantage of $1 / 256$ between the LSB and the output, as calculated by applying a charge of $q$ to the LSB and obtaining an output charge of $q / 256$. The $100 \mathrm{~nm}$ sensor resolution is effectively scaled down by a factor of 256 in the compliance network. Thus, we obtain superior output-referred resolution by including the sensor in the LSB of the device.

It should be noted that the geometric advantage can also be implemented by connecting a single actuator to its load through a pair of springs (connected similarly to a single unit in the C-2C ladder), but with a stiffness ratio of $1 / 256$. This would result in a more compact design than the MEMDAC. Such a design pattern would result in improved output-referred sensor resolution and enhanced outputreferred stiffness at the expense of reduced deflection. 
A finite element model of the compliance network was implemented in Coventorware to predict the integral and differential nonlinearity error (INL and DNL), (figure 7). This model assumed no mismatch in the beam stiffness. Due to the long computation time of the solver, a limited number of input vectors were simulated with FEA. A lumped element model in the electrical domain was implemented using Cadence. This model accounts for stiffness mismatches. Results of both models are included with measured results using a stroboscopic motion analysis system, and are shown to capture the nonlinearities that were measured (Figure 61). The maximum measured DNL is 2.3 LSB and the INL was measured at 1.9 LSB.

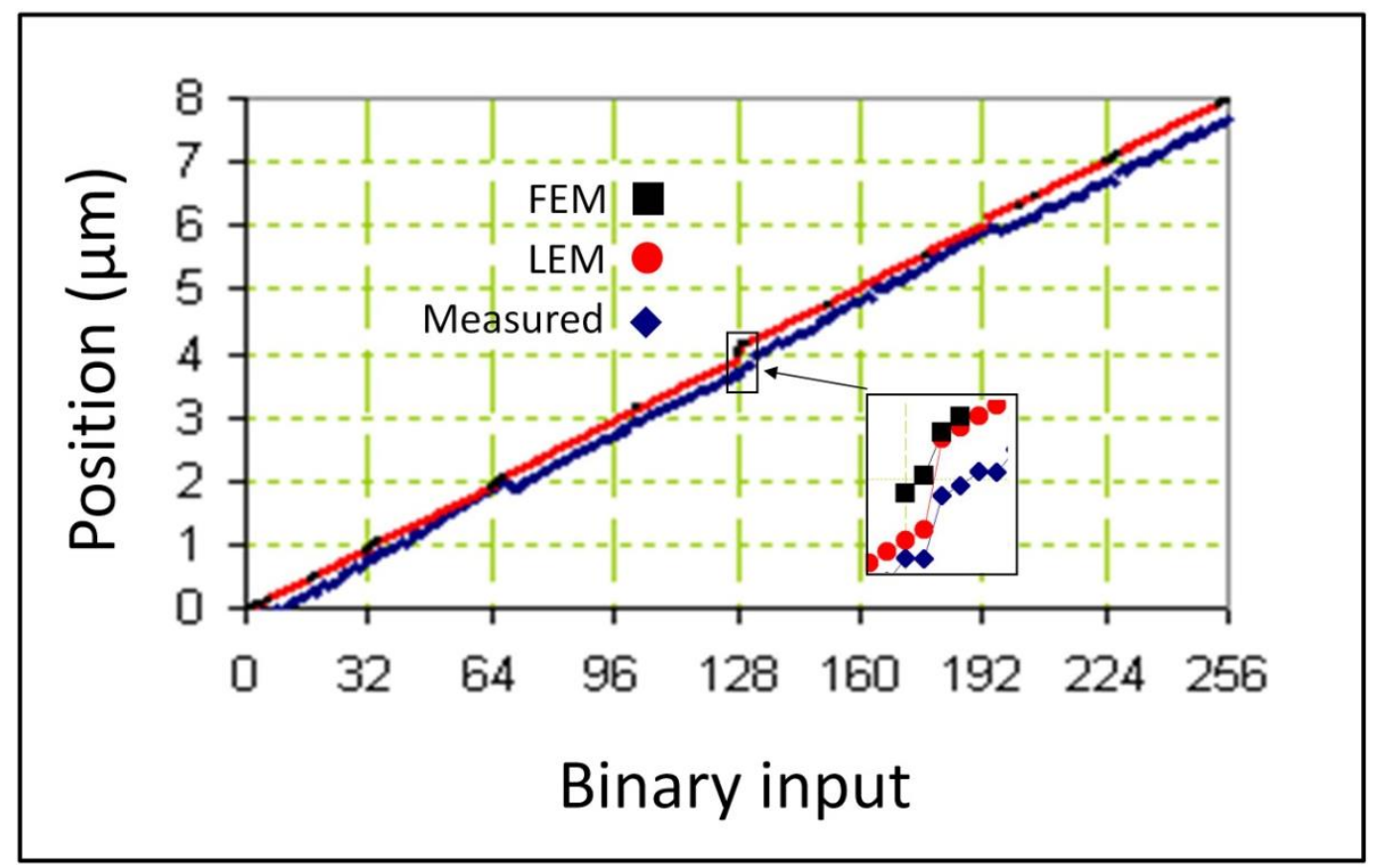

Figure 61: Finite Element Model, Lumped Element Model and measurement results. Significant DNL error occurs at the transition from 127 to 128.

Several processing and design aspects of the device contribute to the observed positioning error. Structural bloat that occurs during the post-CMOS release process reduces the range of motion of the actuators. The asymmetry in the $\mathrm{C}$ and $2 \mathrm{C}$ beams contributes to a compliance mismatch that results from a difference in loading effects during etching. Thermal coupling through the ground plane, substrate and compliance network results in parasitic actuation. This effect is most noticeable in the transition from input value 127 to 128 , since these are the two adjacent values with the largest difference in power consumption and temperature. 
An orthogonal arrangement of four 1-D MEMDACs was used to position a centrally mounted cantilever in 2-D (Figure 62). The device can be positioned on a 2-D grid with a $32 \mathrm{~nm}$ step size, and exhibits similar INL and DNL. It is possible to operate the LSB using an analog input to scan within a step while all other bits are fixed to a specific binary input value. Thus, the rationale behind this device is to position a tip on a 2-D grid quickly with only digital inputs, and scan within each cell in an analog fashion.
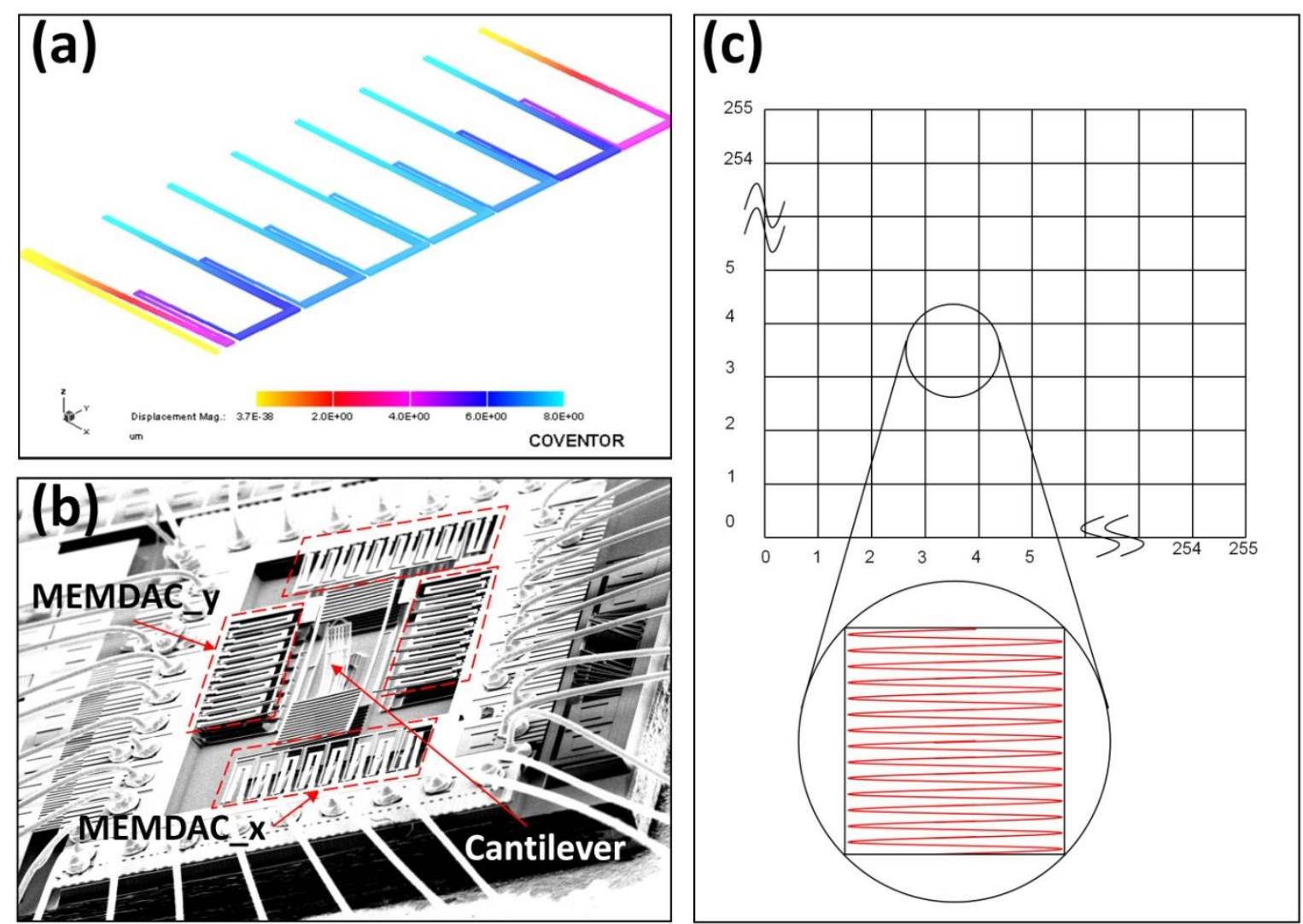

Figure 62: Coventor simulation of the compliance network in a state corresponding to input [01111110] (a). The MSB is the right-most bit connected to the output shuttle, and the LSB is the leftmost bit. A 2D MEMDAC-based Scanner (b). The concept of performing a fine scan using the LSB in a grid defined by the higher bits (c).

To summarize, an 8-bit MEMDAC based on the R-2R electronic DAC architecture was fabricated using a CMOS-MEMS process. The step size achievable with this design is $32 \mathrm{~nm}$, which is smaller than the resolution attainable with a lateral differential piezoresistive position sensing scheme. By placing a strain gauge at the LSB of the device, sub-nanometer position resolution was obtained with measurement levels above the thermal noise floor. Although sub-step positioning operation exhibits good linearity, significant error is observed during transitions in which a large number of bits are toggling and changing the power consumption and heat generation within the device. The footprint of 
a 2-D MEMDAC is much larger than a single lateral actuator. If analog inputs are available for each bit, a 2-bit version of the MEMDAC with capacitances of C-2C replaced by C-256C could provide the same resolution enhancement for the LSB and the same position range as the 8-bit MEMDAC, in a much smaller footprint.

\subsubsection{MEMDAC Thermomechanical Noise}

Thermomechanical noise that is introduced by the network between the LSB and the output may limit the achievable resolution of the device, for a large number of bits. For an 8-bit MEMDAC, thermomechanical noise is lower than the desired resolution. One may use the concept of noise bandwidth [149] to calculate the mean square noise in a cantilever by following the same procedure that is used to calculate Johnson noise in a capacitor, because both are white noise sources. The origin of both sources of noise is thermal fluctuations that bring the capacitor (or spring) into thermodynamic equilibrium with the thermal reservoir.

To calculate the noise bandwidth, a white noise source with spectral density $S_{n}(f)=4 k_{B} T R$ (representing the Johnson noise in a resistor R) is placed at the input of a noiseless RC circuit. The spectral density function at the output of the circuit is:

$$
S_{o}(f)=|H(j 2 \pi f)|^{2} \times S_{n}(f)
$$

The mean square noise at the output is the integral of the spectral density function:

$$
\overline{v_{o}^{2}}=\int_{0}^{\infty}|H(j 2 \pi f)|^{2} \times S_{n}(f) d f=4 k_{B} T R \int_{0}^{\infty}|H(j 2 \pi f)|^{2} d f
$$

The noise bandwidth is the effective bandwidth that determines the mean-square noise due to a white noise source:

$$
\Delta f=\int_{0}^{\infty}|H(j 2 \pi f)|^{2}=\int_{0}^{\infty} \frac{1}{1+(2 \pi f R C)^{2}} d f=\frac{1}{4 R C}
$$

Where the integral was evaluated using:

$$
\int \frac{d x}{x^{2}+1}=\tan ^{-1} x
$$

The mean square noise on the capacitor is therefore: 


$$
\overline{v_{o}^{2}}=4 k_{B} T R \Delta f=\frac{k_{B} T}{C}
$$

The RMS charge noise may be calculated from $Q_{R M S}=C v_{R M S}=\sqrt{k_{B} T C}$. A MEMDAC with thermomechanical noise limited performance would have a number of bits that can be calculated by setting the output-referred charge from the LSB equal to the RMS charge noise:

$$
Q_{L S B}=\frac{2 C_{e} V_{\text {out }}}{2^{N}}=\sqrt{k_{B} T C}
$$

This MEMDAC was designed with beams of stiffness $k=0.5 \mathrm{~N} / \mathrm{m}$, corresponding to a total device capacitance of $\mathrm{C}=2 \mathrm{~F}$. The rms position noise at room temperature is calculated as

$$
Q_{R M S}=\sqrt{1.3806488 \times 10^{-23} \times 300 \mathrm{~K} \times 2}=9.1 \times 10^{-11} \mathrm{~m}
$$

At the operating temperature of $400 \mathrm{~K}$, the noise increases to $1.05 \times 10^{-10} \mathrm{~m}$. The output referred position resolution of the sensor in the LSB is:

$$
\frac{100 \mathrm{~nm}}{2^{8}}=3.9 \times 10^{-9} \mathrm{~m}
$$

In theory, the number of bits could be extended to $n=9$ before the position sensor becomes thermal noise limited at $400 \mathrm{~K}$, which is the operating temperature of the device when all bits are on. A design with lower positioning range and stiffer beams may be used to increase the number of bits and the position resolution.

\subsection{Temperature Sensing for Position Control}

The most significant disturbance in the tip-position of electrothermally actuated single-chip SPM's is thermal coupling. Because the deflection of a thermal actuator is linearly proportional to its temperature, the local temperature in an actuator is a good measure of its unloaded position. A temperature measurement does not capture external loading forces that may influence the actual position of the device. In addition, temperature measurements do not capture the mechanical dynamics of the actuator; however, they do capture the thermal dynamics that arise from the heat capacity and diffusivity of the constituent materials.

The lateral scanners of the instruments reported here are not exposed to external forces and are operated well below their mechanical resonant frequencies, but may be operated above their thermal 
roll-off frequency. Vertical scanners may be separated into DC positioning actuators and resonant flexures. The DC positioning actuators operate in the same regime as the lateral actuators (quasi-static). For quasi-static and unloaded operation, a local temperature measurement provides excellent position resolution and may be used in a controller to mitigate the effects of thermal coupling. For operation under dynamic and/or loaded conditions, piezoresistive sensors must be used.

The thermal coupling issue will be discussed further when data from static AFM operation is shown in Chapter 6 . The problem is most severe when heat from lateral actuation couples into the vertical actuator and interferes with the tracking of sample topology. When a voltage is applied to the vertical actuator, its resistance changes as a function of the input power. If the input voltage is held constant while a lateral actuator is scanning, thermal coupling causes a resistance variation in the vertical actuator. A Wheatstone bridge circuit may be used to measure the resistance of the actuator to compensate for the effect. In the next section, it will be shown that the signal-to-noise ratio of this measurement technique depends on the drive voltage, implying that the resolution improves as the voltage increases.

\subsubsection{Noise in Temperature-Based Position Control}

There are several noise sources that limit the minimum detectable deflection (MDD) of an actuator using temperature sensing. In the electrical domain, Johnson noise is present in the heater resistor as well as the other resistors in the bridge. The heater resistor also contributes $1 / \mathrm{f}$ noise. The electrical noise is shaped by the thermal RC transfer function, in the same way that thermomechanical noise is shaped by the transfer function of a spring. Because of this thermal domain noise shaping, the output position of the actuator does not respond to noise at frequencies beyond its thermal cut-off. In other words, the thermal domain acts as a low-pass filter and band-limits the noise before it reaches the mechanical domain. A noise bandwidth for the actuator may therefore be calculated for the white noise source; however, the filtered signal is not available in the electrical domain, so the measurement circuit does not benefit from this filtering, even though the actuator transfer function itself does. Similarly, thermomechanical noise contributes a mean square position noise in the mechanical domain that is not available in the electrical signal of this measurement technique. In addition to the noise sources in the bridge, the instrumentation amplifier has input-referred noise specifications for its Johnson noise and $1 /$ f noise.

A schematic of the Wheatstone bridge circuit including noise sources is shown in Figure 63. 


\section{Noise in TCR-Based Position Sensing}

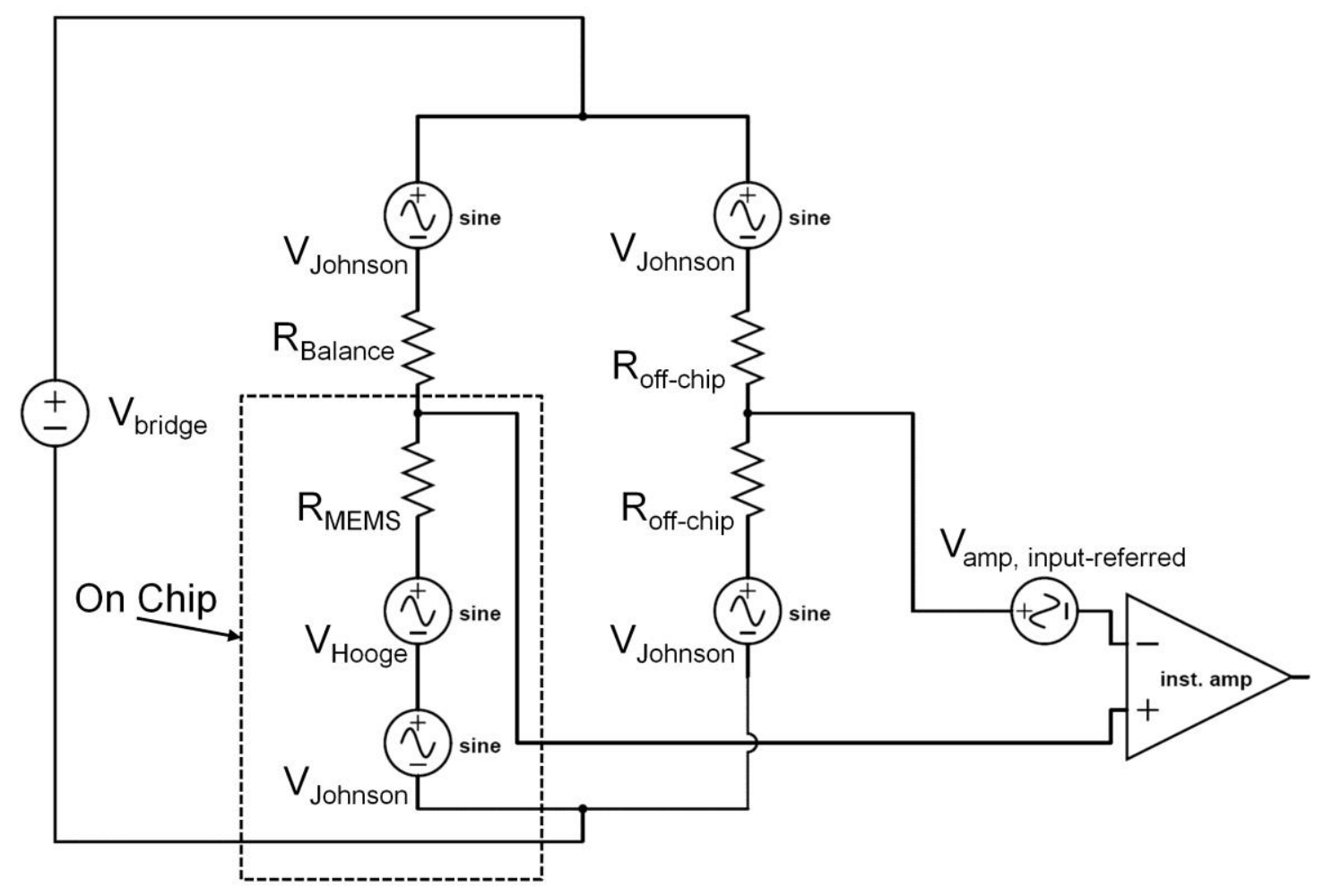

Figure 63: Noise Sources in the Wheatstone Bridge Circuit

The output signal from the bridge is:

$$
V_{o}=V_{\text {in }} \frac{\Delta R}{2(2 R+\Delta R)} \approx V_{\text {in }} \frac{\Delta R}{4 R}
$$

The noise sources in each half of the bridge circuit may be combined and placed in series with a noiseless resistor with a value $R / 2$. The noise voltages of the combined sources are:

$$
v_{n, M E M S}=\frac{v_{n, \text { mems }}+v_{n, \text { balance }}}{2}, v_{n, \text { OFF_CHIP }}=\frac{v_{n, o f f_{-} \text {chip }}+v_{n, o f f_{-} \text {chip }}}{2}
$$

The RMS noise from both halves of the bridge is calculated as the sum of mean-squares:

$$
V_{n, R M S}=\sqrt{\overline{\overline{v n}^{2}}}=\frac{1}{2} \sqrt{\left(\overline{v_{n, m e m s}^{2}}+\overline{v_{n, \text { balance }^{2}}}+2 \overline{v_{n, \text { off_chlp }^{2}}{ }^{2}}\right)}
$$

The mean-square Johnson noise from each resistor is: 


$$
\overline{v_{n, J o h n s o n}{ }^{2}}=4 k_{B} T R \Delta f
$$

In order to lower Johnson noise, the off-chip resistors should be low value resistors. In addition, it is desirable to use higher wattage resistors to minimize prevent self-heating. The balance resistor must match the value of the MEMS resistor, but will contribute less noise because it remains at room temperature. For a given bandwidth, the numerical value of the mean-square Johnson noise in the bridge may be calculated with:

$$
\overline{v_{n, \text { Johnson }}{ }^{2}}=4 k_{B} \Delta f\left[T_{\text {room }}\left(2 R_{\text {off_chip }}+R_{\text {balance }}\right)+T_{M E M S} R_{M E M S}\right]
$$

From equation (5.15), it can be seen that the Johnson noise depends on the temperature of the MEMS device. Assuming a temperature of $450 \mathrm{~K}$, a MEMS resistor value of $150 \Omega$, balance resistor values of $100 \Omega$, and a bandwidth from $1 \mathrm{~Hz}$ to $1 \mathrm{kHz}$, the numerical value of the mean square Johnson noise is calculated as $9.48 \times 10^{-15}$, and the RMS Johnson noise is $97.4 \mathrm{nVrms}$. These values correspond to the heater resistor in a lateral actuator that has a $10 \mu \mathrm{m}$ range of motion, and experiences a $15 \Omega$ resistance change over this range.

The dominant source of $1 / \mathrm{f}$ noise in silicon is Hooge noise [164] which has a spectral density of:

$$
S_{n, H}=\frac{\alpha_{H} V_{b i a s}^{2}}{N f}
$$

Where $\alpha$ is the experimentally measured Hooge parameter and $N$ is the number of carriers. For CMOS polysilicon, $\alpha_{H}$ has been measured as $1.26 \times 10^{-6}$ in [179]. The resistivity is calculated from the sheet resistance and thickness values of the polysilicon in the foundry design kit, and is found to be $9.00 \times 10^{-4}$ $\Omega$-cm. From [180] and [181], this resistivity value corresponds to a carrier concentration that is $>10^{19} \mathrm{~cm}^{-}$ 3. The number of carriers in a $150 \Omega$ heater resistor with dimensions of $10 \mu \mathrm{m} \times 30 \mu \mathrm{m}$ is calculated to be $5.4 \times 10^{8}$. The mean-square Hooge noise over a bandwidth from $1 \mathrm{~Hz}$ to $1 \mathrm{kHz}$ is:

$$
\overline{v_{n, \text { Hooge }}{ }^{2}}=\frac{\alpha_{H} V_{\text {bias }}{ }^{2}}{N} \ln \left(\frac{f_{\text {max }}}{f_{\min }}\right)
$$

When the bridge is balanced, $V_{\text {bias }}=V_{\text {in }} / 2$. Assuming a $1 \mathrm{~V}$ drop across the heater resistor, the calculated value of mean square Hooge noise in the $150 \Omega$ resistor is $40.3 \times 10^{-15}$ and the RMS Hooge noise is $20.1 \mathrm{nVrms}$. The RMS Hooge noise scales linearly with the applied voltage, but remains below the Johnson noise in this example. If the resistor dimensions were chosen to be $2 \mu \mathrm{m} \times 6 \mu \mathrm{m}$, 
maintaining the same resistor value but reducing the number of carriers by a factor of 25 , the Hooge noise would surpass the Johnson noise at $\sim 0.65 \mathrm{~V}$. Thus, the largest possible dimensions should be chosen to reduce Hooge noise, and a low resistance should be chosen to reduce Johnson noise.

The value of the input-referred noise of the instrumentation amplifier may be obtained from component datasheets. Since the input impedance represented by the bridge is low, an instrumentation amplifier with low input-referred voltage noise is preferable, while the current noise specification is less important. As an example, the INA103 from Texas Instruments has an input-referred RMS noise voltage of $62.6 \mathrm{nVrms}$ when set to a gain of $100 \mathrm{x}$, considering both $1 / \mathrm{f}$ and Johnson noise terms. The same amplifier at a gain of $1000 x$ has an input-referred RMS noise voltage of $264.7 \mathrm{nVrms}$. The range of noise values implies that none of the noise sources can be ignored. The RMS thermomechanical noise for an actuator with a moderate stiffness of $10 \mathrm{~N} / \mathrm{m}$ is $2.04 \times 10^{-11}$, which is well below all of the other noise sources considered here. Therefore, the fact that this sensing method cannot detect the applied thermal noise force is not relevant to its MDD.

The signal to noise ratio of the temperature sensing circuit is:

$$
\frac{\overline{V_{o}^{2}}}{\overline{V_{n}^{2}}}=\frac{\left(V_{\text {in }} \frac{\Delta R}{4 R}\right)^{2}}{4 k_{B} \Delta f\left[T_{\text {room }}\left(2 R_{\text {off_chip }}+R_{\text {balance }}\right)+T_{M E M S} R_{M E M S}\right]+\frac{\alpha_{H} V_{\text {in }}{ }^{2}}{4 N} \ln \left(\frac{f_{\text {max }}}{f_{\text {min }}}\right)+\overline{v_{A}{ }^{2}}}
$$

The objective of the circuit is to ascertain the position of the actuator based on a measure of the heater resistor value, which depends on temperature. To quantify the dependence of the signal-to-noise ratio on the input voltage, it is necessary to express all of the variable quantities in equation (5.18) as a function of $V_{i n}$. This may be done in the self-heating case, since the temperature is a direct result of the power dissipated in the device. The concept of a power coefficient of resistivity $\left(\mathrm{PCR}\right.$, or $\left.\alpha_{P}\right)$ is used to transform the quantities. Two identical actuators with the same coefficient of resistance may experience different amounts of self-heating under the same input voltage, because their thermal isolation from the substrate may vary. The efficiency of the actuator is a measure of its output deflection (and temperature) with respect to input power, which may be improved via thermal isolation. It will be shown here that the sensitivity of the TCR-based measurement circuit is compromised when the efficiency is increased.

The PCR may be defined in a similar manner to the TCR: 


$$
R(P)=R_{0}+R_{0} \alpha_{P} P
$$

Thus, the power efficiency of the actuator may be defined as:

$$
\eta_{P}=\frac{\Delta T}{\Delta P}=\frac{\alpha_{P}}{\alpha_{T}}=\frac{\Delta T R_{0}}{V_{\text {bias }}^{2}}
$$

For a given bandwidth, at room temperature, equation (5.18) can be expressed as a function of only $V_{\text {in }}$ :

$$
\begin{aligned}
& \overline{\overline{V_{o}{ }^{2}}} \\
& =\frac{\left(\frac{V_{\text {in }}{ }^{2} \eta_{P} \alpha_{T}}{8 R_{M E M S}}\right)^{2}}{4 k_{B} \Delta f\left[\frac{T_{\text {room }}}{V_{\text {in }}{ }^{2}}\left(2 R_{\text {off_chip }}+R_{\text {balance }}\right)+R_{M E M S}\left(T_{\text {room }}+\frac{\eta_{P}}{4 R_{M E M S}}\right)\right]+\frac{\alpha_{H}}{4 N} \ln \left(\frac{f_{\text {max }}}{f_{\text {min }}}\right)+\overline{v_{A}{ }^{2}}}
\end{aligned}
$$

The values for the various constants in equation (5.21) are used to plot the signal to noise ratio as a function of driving voltage shown in Figure 64 . At $\sim 15 \mathrm{mV}$, the signal voltage surpasses the total noise in the system, setting the minimum detectable resistance change to $8.9 \mathrm{~m} \Omega$, and the minimum detectable temperature change to $59 \mathrm{mK}$. For the lateral actuator in question, this corresponds to a $5.9 \mathrm{~nm}$ MDD.

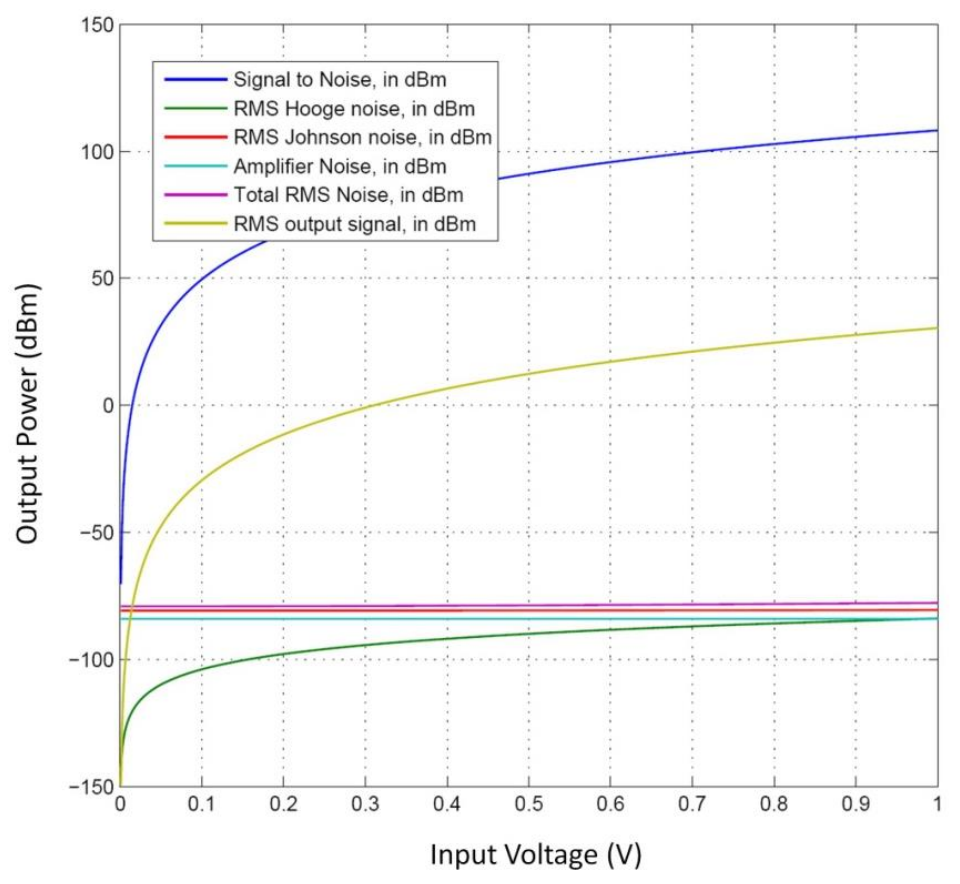

Figure 64: Signal-to-Noise Ratio vs. Input Voltage in Temperature-Based Position Sensing 


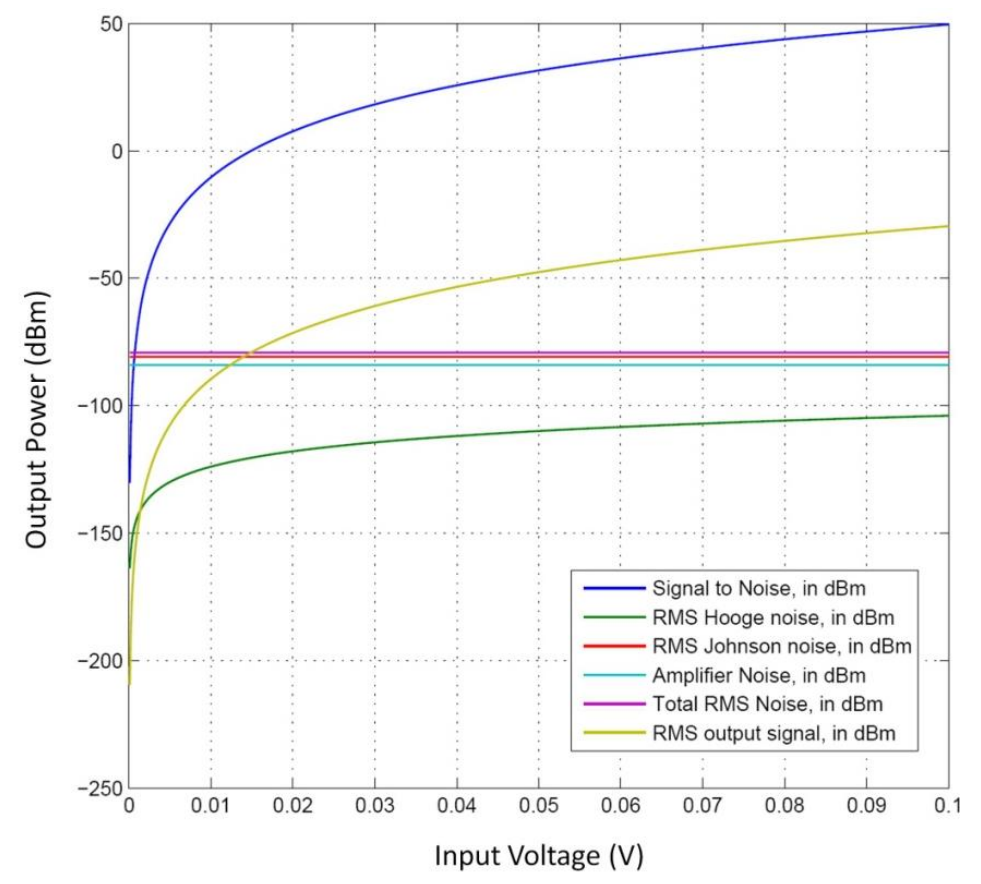

\section{Figure 65: Magnified Plot of Signal-to-Noise Ratio vs. Input Voltage in Temperature-based Position Sensing}

If the efficiency of the actuator is improved by the addition of thermal isolation, a lower voltage results in a higher temperature change. Both the Johnson noise and Hooge noise are scaled up by temperature, while the bridge voltage is reduced. In this example if the TCP is doubled, the new minimum detectable resistance change is $11 \mathrm{~m} \Omega$ and the minimum detectable temperature change is $75 \mathrm{mK}$, corresponding to a MDD of $7.3 \mathrm{~nm}$.

The results of this noise analysis suggest that an actuator with a short thermal path to ground is preferred, and the signal-to-noise ratio improves at higher drive voltages. In other words, increased power dissipation is the cost of higher position resolution. In most temperature sensors, self-heating is an undesirable parasitic effect; however, in this work, the self-heating is the measurand.

The results of this analysis also suggest that the PWM and $\Sigma \Delta$ techniques offer improved resolution. The pulses used in these techniques are much shorter than the thermal time constants, so the actuator never reaches a steady-state temperature that corresponds to the full-scale voltage. In addition, the bandwidth of the measurement circuit discussed here may be increased so that the measurement can be taken within a PWM or $\Sigma \Delta$ pulse. If the bandwidth is increased to $100 \mathrm{kHz}$, the noise increases such that the minimum detectable resistance change occurs at $25 \mathrm{mV}$; however, the measurement voltage applied to the bridge will always be at the full-scale voltage of the digital drive scheme, which is well 
above this detection threshold. A low-pass filter at the output of this circuit may then be used to reduce the bandwidth back to $1 \mathrm{kHz}$.

An AC bridge circuit was used to demonstrate temperature-based position sensing, with the same as in Figure 63, but with an $A C$ voltage supply in series with the bridge voltage. The $A C$ signal is used to measure the resistance, while a DC bias is applied to actuate the device. Upon self-heating, the measured resistance tracks the position of the device as shown in Figure 66.

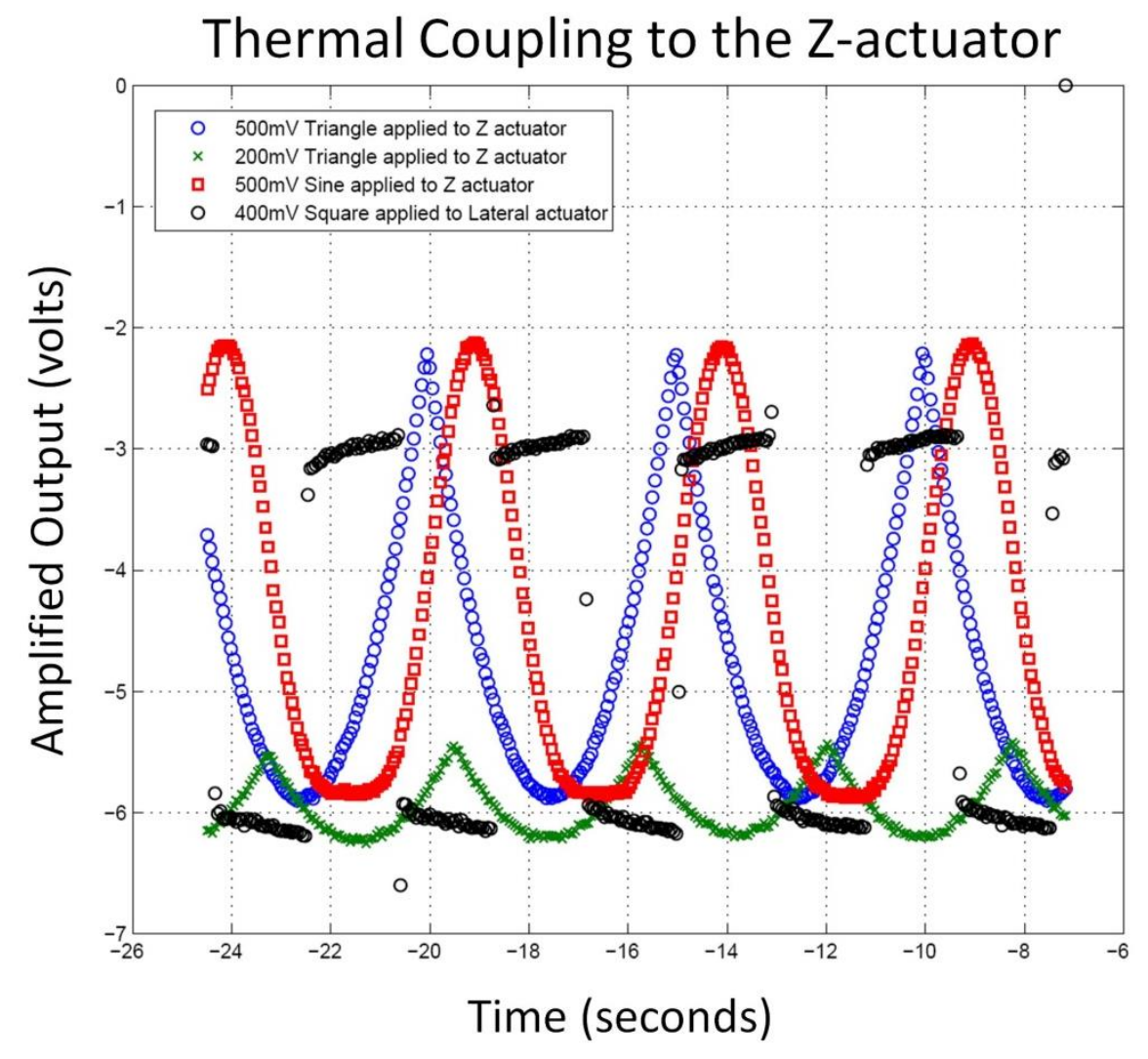

Figure 66: TCR-Based Position Sensing in the Vertical Actuator

The self-heating of the vertical actuator is shown under various input voltages and waveforms. When a triangle wave is applied to the input, the $\mathrm{V}^{2}$ dependence of position is apparent. When the vertical actuator is off and a lateral actuator is periodically powered with a square wave, thermal coupling to the vertical actuator is measured. The thermal dynamics are captured by this method, as is evident in the sloped profile of the vertical actuator's response to heat from the lateral actuator. It should be noted that the lateral actuator resistance is $\sim 150 \Omega$, while the vertical actuator resistance is $\sim 900 \Omega$. Thus, an input voltage of $400 \mathrm{mV}$ to the lateral actuator represents $\sim 4 \mathrm{X}$ more power than $500 \mathrm{mV}$ in the vertical 
actuator. This is why the magnitude of the parasitic coupling signal is similar to the self-heating signal. Nevertheless, this amount of coupling clearly changes the position of the vertical actuator, which is problematic during scanning operation.

A PID controller is used to effectively mitigate the thermal coupling. In this configuration, the vertical actuator must always be at a temperature that is above the maximum temperature that may result from thermal crosstalk. This limits the range of the actuator, but allows the controller to compensate for heat flow from lateral actuators. The performance of the controller is shown in Figure 67.

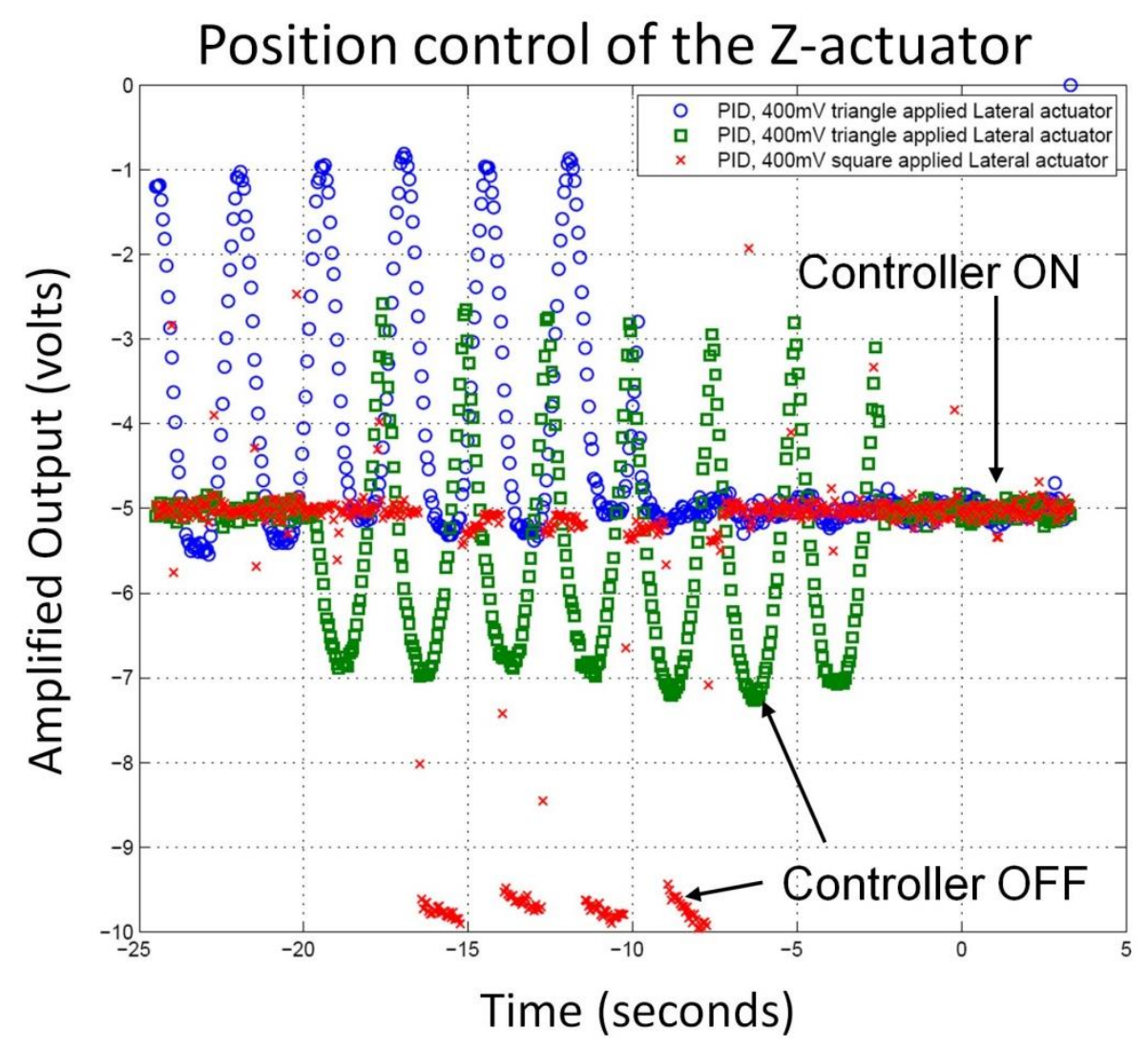

Figure 67: PID Control of the Vertical Actuator in the Presence of Thermal Coupling

The temperature-based deflection sensing methods discussed in this section may be used to realize closed-loop scanners with nanometer-scale position resolution. The feature is desirable in metrology and patterning applications, and it has been implemented at the macro-scale for conventional SPM's using capacitive and optical interferometry techniques. The technique is appropriate when external loading forces may be neglected, and the mechanical dynamics of the scanner are not in the frequency 
band of interest. When the intention is to detect small forces from tip-sample interaction or to control the mechanical dynamics of a cantilever, piezoresistive detection is a suitable option.

\subsection{Piezoresitive detection}

The noise that limits the force resolution of piezoresistors arises from the same sources as those discussed above: electrical Johnson noise, electrical 1/f noise (Hooge noise), thermomechanical noise, and amplifier noise. Unlike temperature sensors, piezoresistors can detect signals in the mechanical domain, which means that thermomechanical noise has the potential to influence the output signal. On the other hand, the temperature in the resistor is now a parasitic source that contributes unwanted noise to the force signal, and lowers sensitivity by way of the negative temperature coefficient of piezoresistivity.

The design and optimization of piezoresistive cantilevers is a multivariable problem that has been approached with different constraints. For the u-shaped design in [164], the authors optimized the $1 / f$ noise performance by controlling the thickness, leg length ratio, and width of the cantilever; the depth and doping concentration of the piezoresistor; and the surface treatment and anneal. In [182], parameters were optimized to improve the minimum detectable force. In a CMOS process, the doping concentration, thickness of the piezoresistor, and the thickness of the cantilever is set by the foundry. In addition, the desired stiffness is constrained by the type of tip-sample interaction that is to be measured. The design space is therefore limited to the 2-D layout of the device. A reasonable approach is to begin with the narrowest beam allowed in the process, increasing the strain in response to an input force. Since the region of highest stress concentration is near the base of the cantilever, the piezoresistor should be aligned with the base. At this point, the length of the piezoresistor should be increased until the Hooge noise and Johnson noise are balanced. The gauge factor and strain values may then be used to calculate the minimum detectable deflection or force.

Although the same principles may be applied to optimize cantilevers for dynamic AFM, the Q-factor enhancement methods discussed in section 4.3 provide better sensitivity than a CMOS-MEMS piezoresistor optimized for force sensitivity. Interestingly, the placement and sizing of polysilicon for Qpumping would result in poor static sensitivity. The drive methods used for Q-enhancement would also be ill-advised if the goal was to improve strain sensitivity. 
This section focuses on drive and sense concepts relating to the static deflection sensitivity, for use in contact AFM. First, the signal source and noise sources are considered. Then, several other parasitic effects are introduced, along with methods to counter them.

\subsubsection{Piezoresistor Signal to Noise Ratio}

A static AFM cantilever with an integrated piezoresistive sensor is shown below in Figure 72 . The cantilever is $100 \mu \mathrm{m}$ long and $3 \mu \mathrm{m}$ wide, and has a stiffness of $\sim 0.2 \mathrm{~N} / \mathrm{m}$. The strain in the piezoresistor results from residual stress, stress from thermal actuation, and stress from tip-sample interaction forces. A balanced design is assumed to compensate for the residual and thermal contributions, so this analysis considers the tip-sample interaction force as the only contributor to the output signal from the bridge. The strain may be calculated from equation (4.26) which evaluates to:

$$
\epsilon_{x_{a v g}, t-s}=\frac{F_{t-s}}{2(E I)_{e f f, y}}\left[t_{p z r}+2 z_{0}-2 z_{c}\right]\left(2 L_{c a n t}-2 x_{0}-L_{p z r}\right)=F_{t-s} K_{t-s}
$$

Out of the quantities that affect the strain, only $L_{c a n t}, 2 x_{0}, L_{p z r}$ and $(E I)_{e f f, y}$ can be controlled in the design process. The width of the beam is the controllable parameter that influences $(E I)_{e f f, y}$. The thickness and vertical placement of the piezoresistor are set by the fabrication process for the thinnest beam geometry available. The gauge factor for the polysilicon in this process is experimentally determined to have a value of -10 . From equation (4.16), the change in resistance can be calculated as:

$$
\Delta R=R \times \epsilon \times G F_{l}
$$

The output voltage from the bridge circuit is:

$$
V_{o}=V_{\text {in }} \frac{\Delta R}{2(2 R+\Delta R)} \approx V_{\text {in }} \frac{\Delta R}{4 R}
$$

The noise is calculated using the equations from section 5.3.1. This noise model includes self-heating effects, Hooge noise calculated from the geometry of the resistor and its bias voltage, Johnson noise calculated from the value of the resistor and self-heated temperature, and the amplifier noise. The RMS displacement corresponding to the thermomechanical noise of the cantilever is $1.5 \times 10^{-10}$, which is near the detection threshold of these devices, as shown in the plot of Figure 68. In the plot, the length of the piezoresistor was scaled from $10 \mu \mathrm{m}$ to $30 \mu \mathrm{m}$, while the cantilever length was held constant at $100 \mu \mathrm{m}$. The MDFs of the $10 \mu \mathrm{m}, 20 \mu \mathrm{m}$ and $30 \mu \mathrm{m}$ cantilevers are $110 \mathrm{pN}, 85 \mathrm{pN}$, and $80 \mathrm{pN}$ repectively. These figures correspond to mimimum detectable deflections of $450 \mathrm{pm}, 480 \mathrm{pm}$, and $620 \mathrm{pm}$. During contact 
mode imaging, the cantilever will therefore need to be pre-loaded to these deflections, and will experience the corresponding forces at the tip. As a point of reference, the force exerted on the tip by meniscus forces is typically on the order of $100 \mathrm{nN}$, which is easily detected by these cantilevers. Conventional AFM imaging is usually carried out at a force set-point of $\sim 1 \mathrm{nN}$, which near the limit of the position resolution of the cantilevers in consideration here.

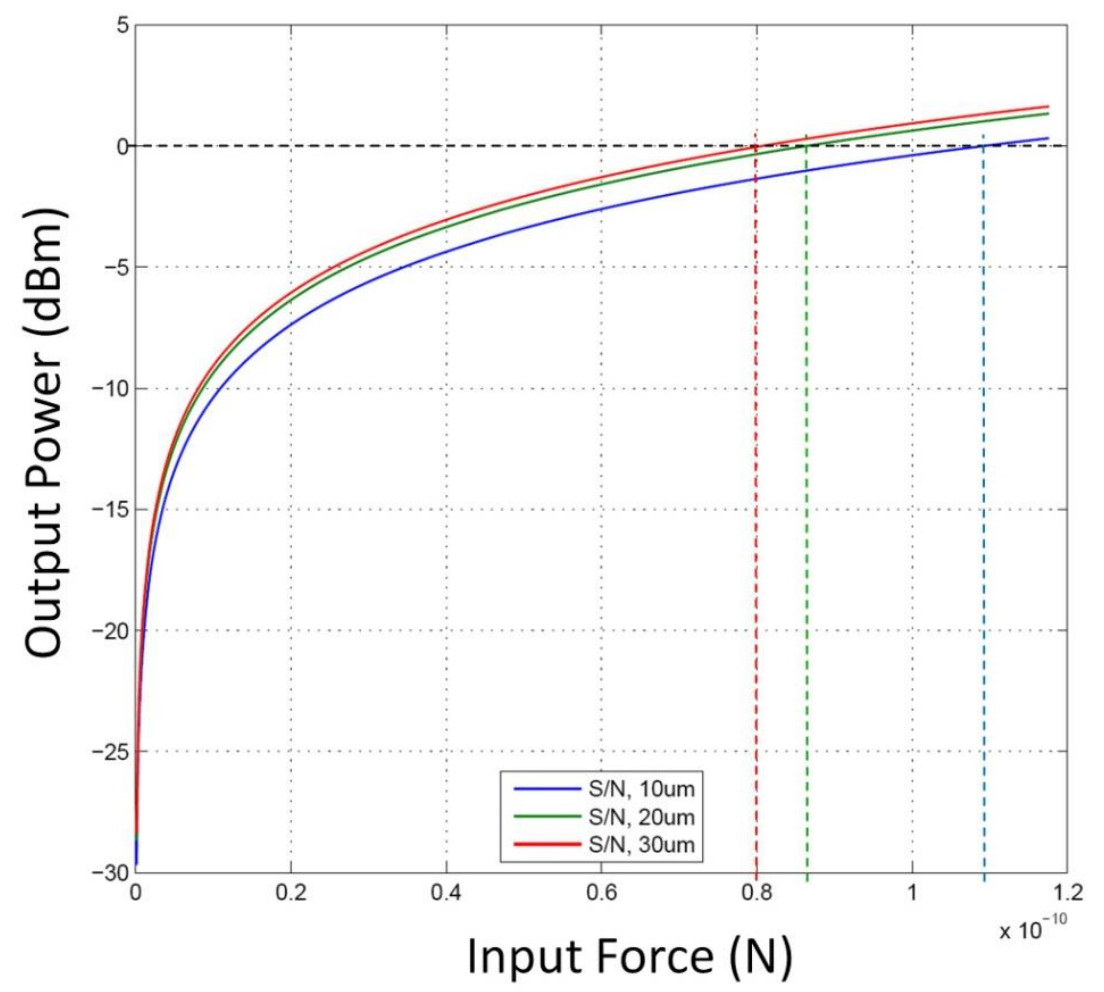

Figure 68: Minimum Detectable Force for Various Piezoresistor Lengths

It is tempting to reduce the size of the piezoresistor in order to place it in a region of higher stress within the cantilever. However, this reduces the number of carriers in the resistor, and hence increases the Hooge noise. Figure 69 shows that for small resistors, the signal power $V_{O, R M S}$ is relatively constant but the noise increases, such that the overall SNR is dominated by noise. If the resistors are sized too large, the converse is true: the noise power remains relatively constant while the signal power decreases, due to a lower average strain captured by the piezoresistors. Figure 70 illustrates the noise behaviour of larger resistors, where the SNR is dominated by changes in $V_{o, R M S}$. 


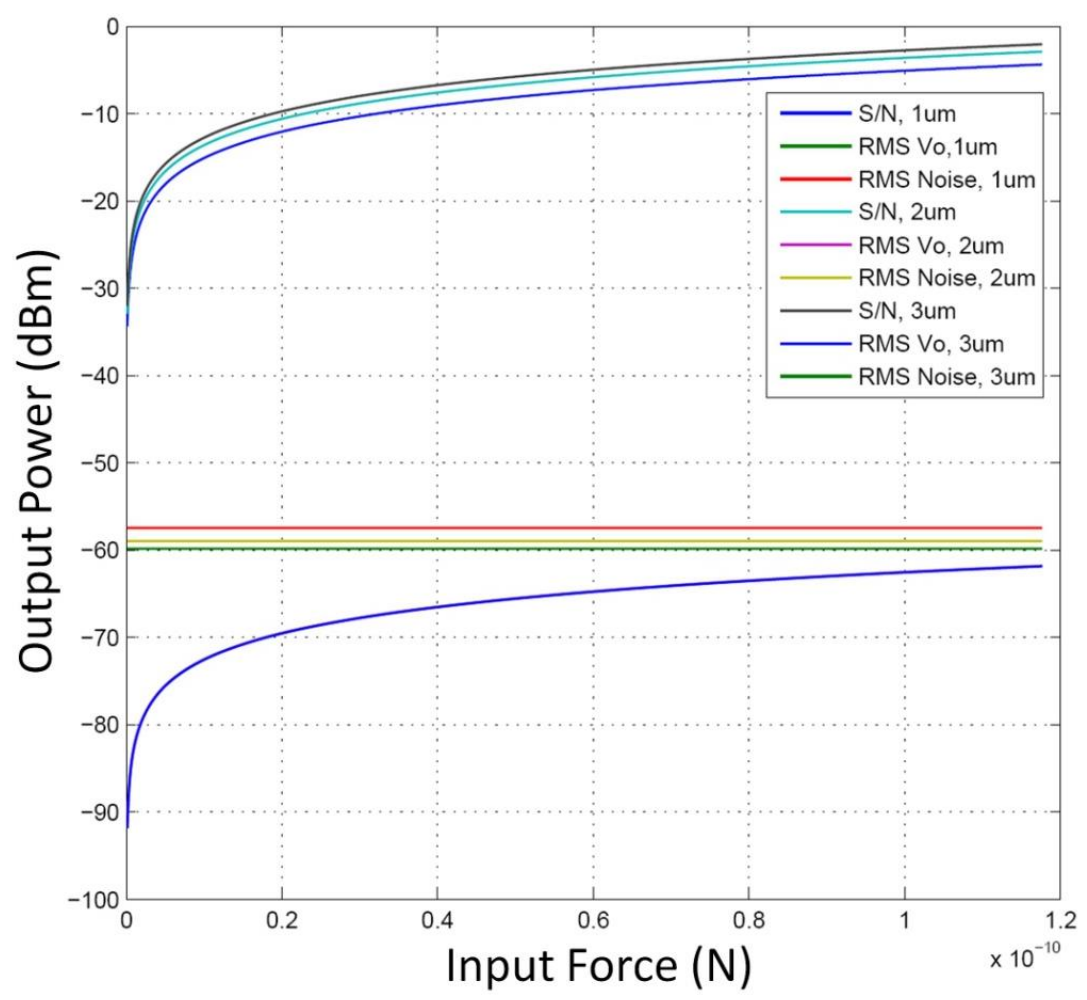

Figure 69: Signal-to-Noise Ratio for Short Piezoresistors

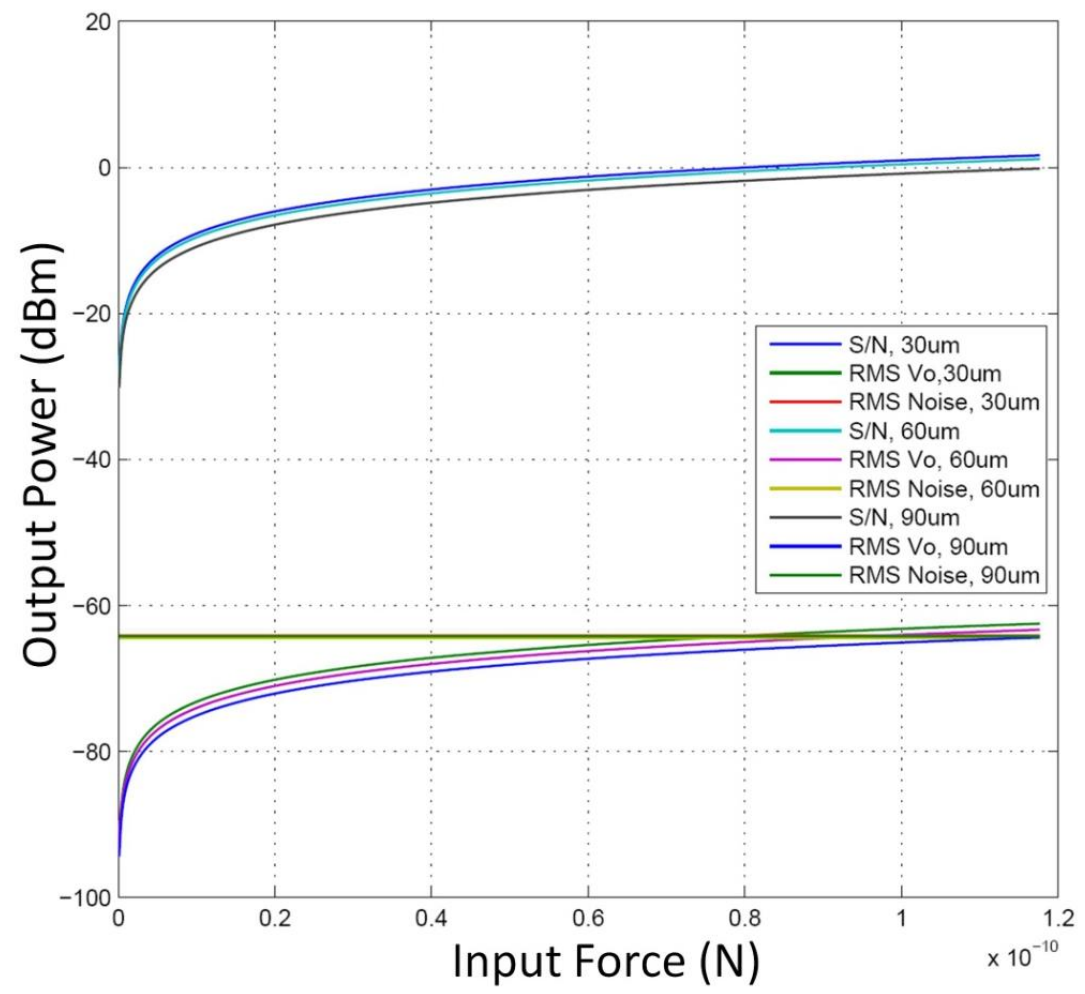

Figure 70: Signal-to-Noise Ratio for Short Piezoresistors 
The fundamental noise limit of a CMOS-MEMS piezoresistive cantilever sets a lower boundary for the MDF, and there is certainly room for improvement in the geometry of the designs that have been implemented thus far. However, the operation of these sensors in a single-chip SPM involves sources of disturbance that are greater than noise sources.

\subsubsection{Other Disturbances}

\section{Ground Bounce}

When the piezoresistive bridge circuit shares a ground connection with the rest of the device, a situation known as "ground bounce" contributes unwanted noise to the bridge output. Figure 71 shows the reason behind this deleterious effect. In a typical device, the piezoresistors may be mounted at the distal end of a vertical actuator. The actuator and piezoresistors share a common path to ground, which may be routed through other actuators along the way. As the vertical actuator is powered, current through this resistive path raises the ground level of the on-chip portion of the bridge output. There is now a small voltage difference between ground levels of the on-chip and off-chip voltage dividers, which is amplified by the gain of the instrumentation amplifier. This component of the signal may be greater than the piezoresistive signal from tip-sample contact. To mitigate this issue, a separate ground may be introduced for the sensors if there is enough room. Alternatively, the configuration shown below on $t$ the right may be used, in which both halves of the bridge experience the same ground level shift.

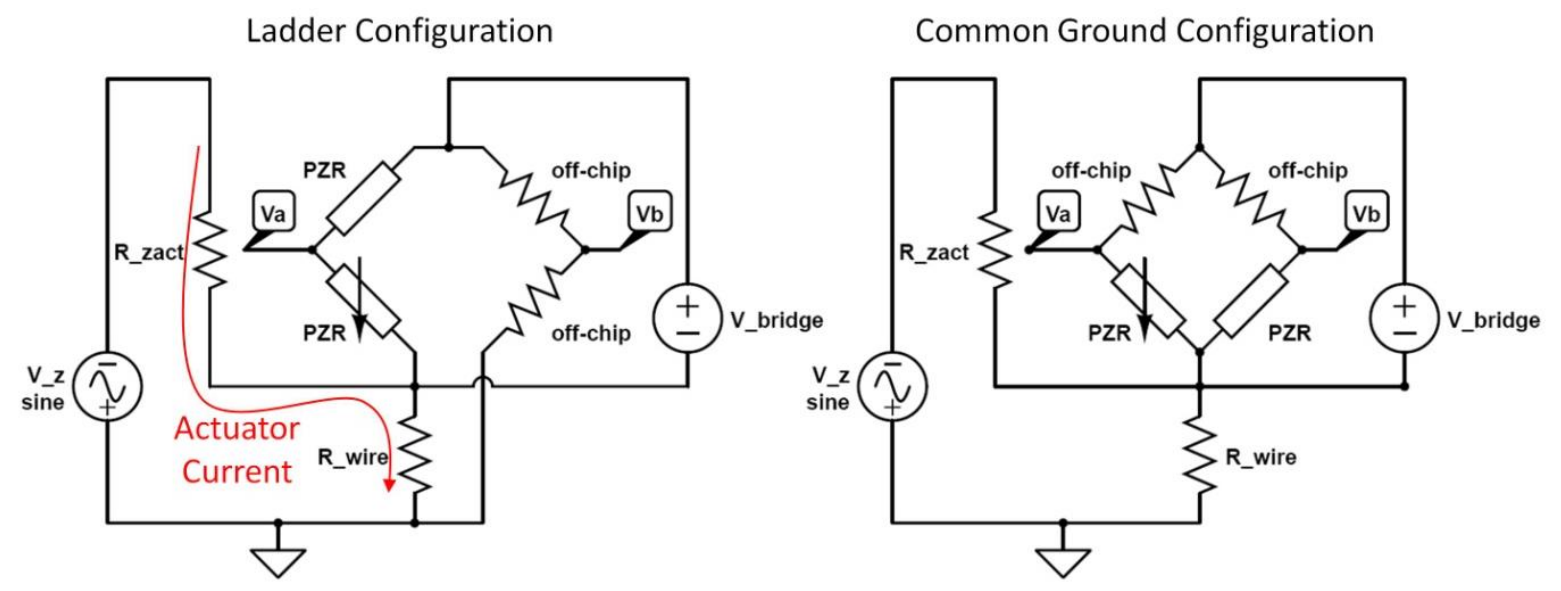

Figure 71: Wheatstone Bridge Configurations.

\section{Quasi-Static Thermal Mismatch}

Thermal coupling between actuators was quantified in section 5.3, where it was shown that a significant amount of heat may couple into the Z-actuator from lateral actuators. The temperature increase is also 
experienced by the piezoresistor in the Wheatstone bridge. The piezoresistor value is influenced by its TCR and by the thermally induced strain in bimorph cantilever. A balanced pair of piezoresistors is used in order to attempt to match the thermal crosstalk so that it appears as a common mode signal in the bridge output. Although the matching is effective in vacuum, it does not sufficiently compensate the effects in air. A closer look at the cantilever layout in Figure 72 reveals that the placement of the piezoresistors is not symmetric with respect to heat flow during imaging.

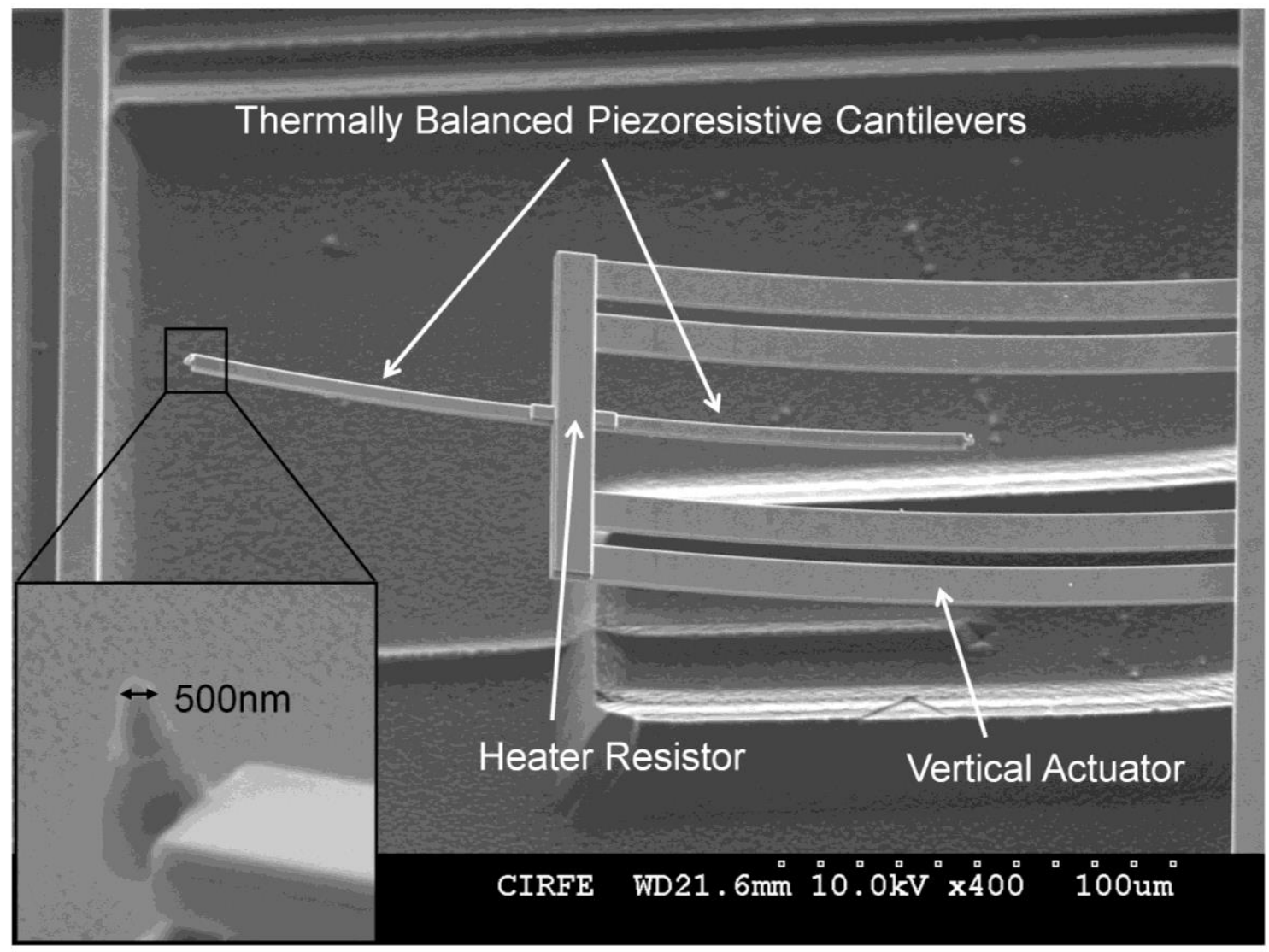

Figure 72: Placement of Balanced Piezoresistors in a Contact AFM Device

The first asymmetry arises due to the difference in the air gap between the substrate and the piezoresistors, due to the out-of-plane angle of the Z-actuator. The gap is large enough $(\sim 25 \mu \mathrm{m})$ that it does not contribute significantly to the cooling of the piezoresistors, when compared to thermal conduction through the remainder of the device. In fact, convection boundary conditions are usually used to model heat loss through gaps of this size. However, the difference between the cooling rates of the two piezoresistors is amplified by a factor of 100-1000x in the instrumentation amplifier. 
The second asymmetry arises due to the difference in the air gap between the sample and the piezoresistors, and it also stems from the angle of the Z-actuator. The thermal conductivity of the path to the sample is proportional to the reciprocal of the gap. At small gaps, there is much more heat flow to the sample from the sense cantilever than from the "dummy" cantilever. Figure 73 shows the amplitude of the output signal under atmospheric conditions, in a vacuum of 10 Torr, and in a vacuum of 0.5 Torr. The vertical actuator is driven by an input voltage, and the output from the piezoresistors bridge circuit is detected by an instrumentation amplifier circuit with a gain of 100 . There is no sample present, and AC modulation of the bridge circuit is not employed.

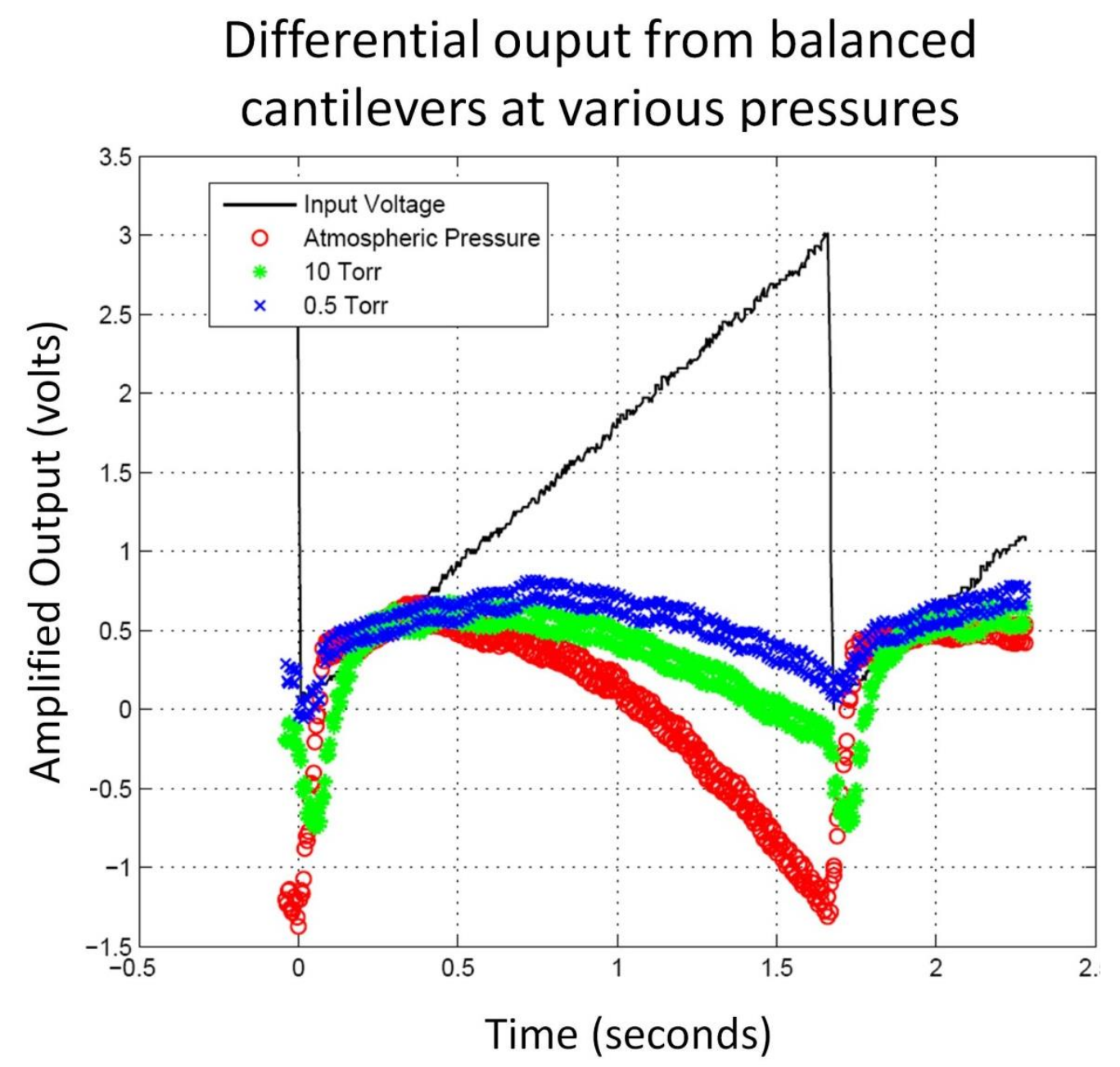

Figure 73: Cantilever Output Under Varying Contact Conditions

The black curve in Figure 73 represents the input signal which is a voltage sawtooth waveform. The red curve represents the difference in temperature between the balanced cantilevers under atmospheric conditions, while the green and blue curves represent the same quantity at lower pressures. Although 
the bridge is not perfectly matched, the behaviour of the device is revealed by these measurements. At ambient pressure, the asymmetry in conduction through the air gap to the substrate results in a large offset voltage at the output of the circuit. As the pressure is reduced, this heat path is removed, and only the asymmetry in the thermal conduction path of the device itself remains. These results validate the notion that most of the cross-talk is related to asymmetries in the air gap, and not due to some inherent asymmetry in the device.

It is important to note that as the pressure is decreased, the temperature in the vertical actuator is significantly increased due to the removal of convection and conduction through the air gap. Thus, the remaining cross-talk under vacuum conditions represents a much larger vertical deflection of the cantilever, indicating that the unbalanced situation improves more than one might initially glean from this plot. In addition, since the cantilever deflects downwards upon heating, it is in closer proximity to the substrate. For a more accurate assessment of the reduction of thermal coupling under vacuum conditions, the vertical actuator resistance would need to be in closed-loop control such that the actuation amplitude would remain constant over pressure.

Another feature in this data is that the dynamics of the cooling process are evident in two regimes. Near the abrupt change in the input voltage, one observes a rapid change in the signal due to the rapid movement of the cantilever away from the substrate and the simultaneous cooling process. At intermediate pressures, the difference between the temperature of the cantilevers is seen to briefly spike, indicating that there is a phase shift between their temperatures. At these pressures, the time constants associated with conduction through the device and conduction through the air gap may be assessed, since their amplitudes are comparable.

Another issue is that the heater for the vertical actuator is placed directly at the base of the piezoresistors in this device. Although the temperature distribution is symmetric in the sensors under vacuum conditions, the SNR in air is lowered dramatically. In subsequent designs the heaters were placed at the base of the Z-actuators, improving the situation. In addition, it is possible to detect higher harmonics from the nonlinearity that results from tip-sample interaction, as discussed in section 6.4. This method rejects thermal coupling by measuring the signal at a frequency that does not contain the thermal coupling signal. 


\section{Reactive Matching}

At sufficiently high frequencies, the RMS amplitude of thermal coupling from the vertical actuators begins to drop due to the RC nature of the thermal circuit. In dynamic AFM using the EARC, the sense cantilever is excited into resonance by the Z-actuator, while the "dummy" cantilever remains stationary. Although the thermal signal is attenuated and the signal from resonance is out-of-phase with the thermal signal, the coupling may still be problematic. If there is a mismatch between the sensors and they are placed in a resistive divider configuration on the same side of the Wheatstone bridge, the output of the circuit will contain a reactive component that cannot be matched with off-chip resistors. The amplitude of the reactive component limits the dynamic range that can be achieved. On the other hand, if the bridge topology from Figure 71 is used, the off-chip resistors could scale the relative amplitude of each half of the bridge for an improved match at a given frequency. At resonance, the sense-piezoresistor phase undergoes a 180 degree shift and crosses the balance piezoresistor's phase value. The bridge may be used such that the amplitudes are matched at this phase cross-over point, to improve the dynamic range of the measurement. The mismatch in the thermal capacitances is required for this method of operation. Alternatively, a Wien bridge configuration with an off-chip capacitor may be used to achieve a wideband match.

\subsection{Summary}

This chapter explored the methods that are used to drive and sense single-chip SPMs. Several driving strategies were introduced to improve the linearity of scanners in both open loop and closed loop configurations. Electrothermal actuation is the dominant source of thermal disturbances in these instruments, so methods to mitigate thermal crosstalk were sought. The MEMDAC provides high resolution displacements that are not limited by thermal or electronic noise. However, its footprint, complexity, stiffness and range of motion make it an unlikely candidate for single-chip SPMs. Digital drive schemes are demonstrated that are easy to implement on off-the-shelf microcontrollers. These schemes may be used in a closed loop fashion with improved resolution over analog resistive temperature sensing, because they measure the resistance at a higher voltage.

The fundamental resolution limitations of resistive temperature sensors and piezoresistors are calculated based on well-known models for the dominant noise sources in the system. If the thermal disturbances can be suppressed, the results of this analysis suggest that the resolution of the instruments can be competitive with large-scale SPMs. 


\section{Chapter 6. Contact AFM}

The first mode of operation that was attempted with single-chip CMOS-MEMS SPM's was contact mode AFM. The nature of the force between the tip and sample is described by the Lennard-Jones potential, which reveals attractive and repulsive regimes that occur at different tip-sample separations. At large separations, attractive forces form electrostatic and Van Der Waals interactions are dominant. When the distance is reduced, a repulsive force with a large gradient appears. Contact-mode AFM operates in the repulsive regime.

\subsection{Device Geometry}

The device geometry of an AFM with integrated 3-D actuation is shown in Figure 74. Two pairs of orthogonally arranged lateral actuators are coupled to a central stage through flexures. The lateral scan range is $10 \mu \mathrm{m} \times 10 \mu \mathrm{m}$. The central stage houses a vertical actuator that can achieve a $25 \mu \mathrm{m}$ displacement. A pair of balanced cantilevers is located at the distal end of the vertical actuator to measure the tip-sample interaction force. The self-heating resistors in the actuators may be used as temperature sensors for closed-loop positioning.

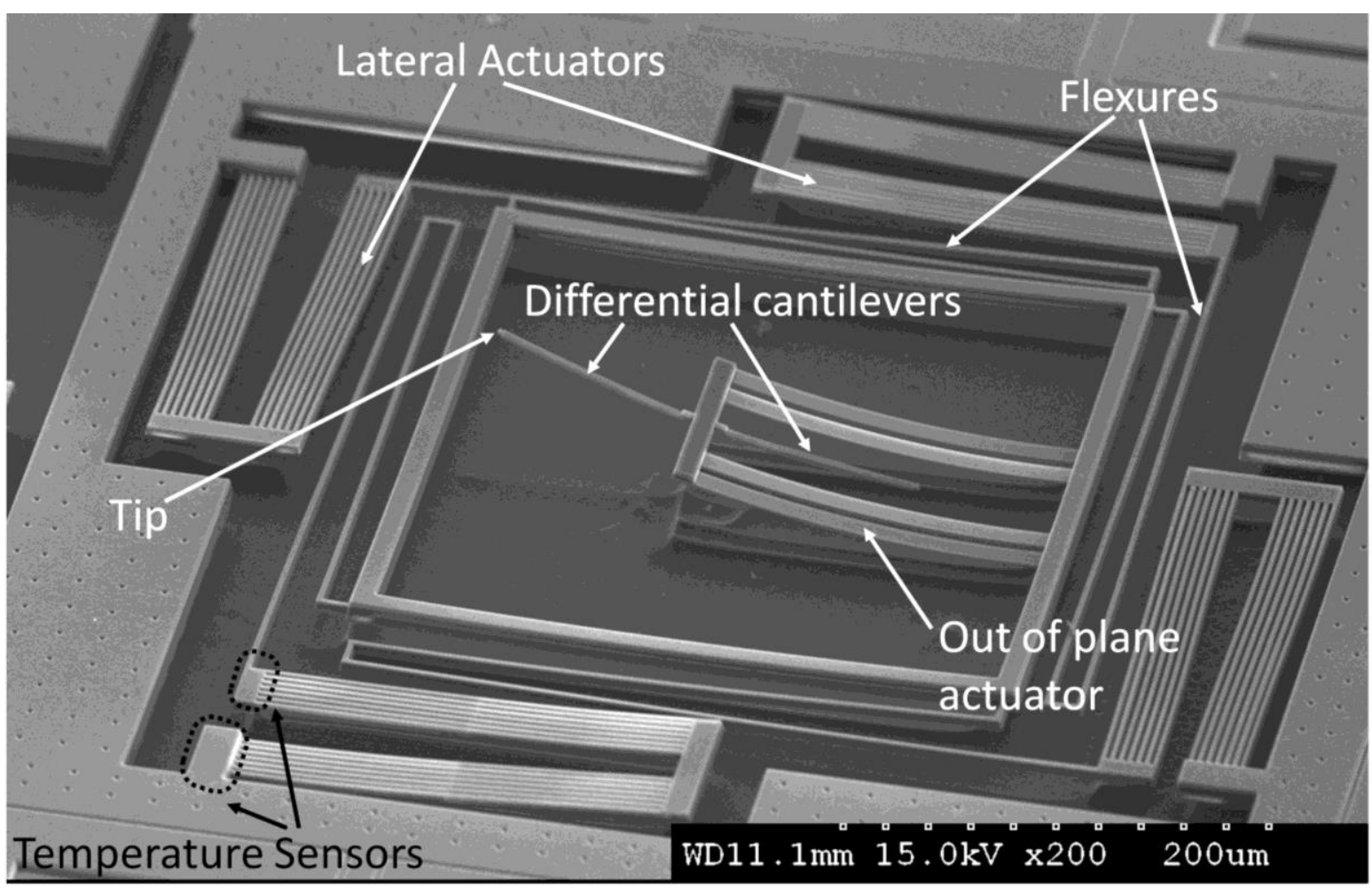

Figure 74: Single-Chip Contact AFM Device Geometry 


\subsection{Tip-Sample Approach Curves}

In order to contact a sample, the tip must self-assemble out of the plane of the chip. A tungsten probe is lowered onto the tip in order to observe the pull-in and snap-out effects that are known to occur in contact AFM. Because the probe is on a manual micromanipulator, the vertical actuator of the device is used to as the approach mechanism. A single cycle of data is shown in Figure 75.

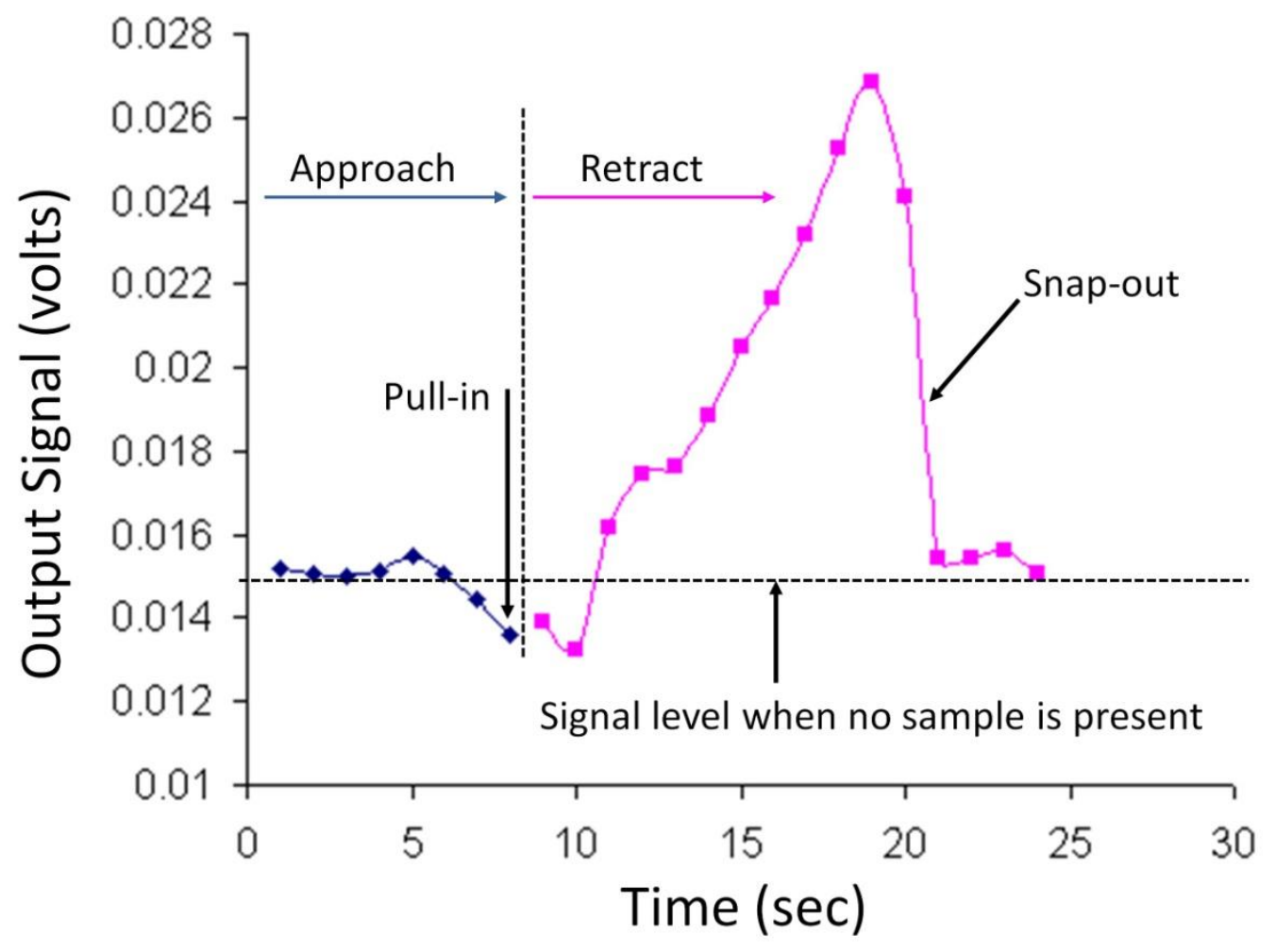

Figure 75: Tip-Sample Approach and Retract Curves

\subsection{Thermal Cross-Talk}

The orthogonal arrangement of actuators and flexures provides decoupling of the motion in the mechanical domain. In the thermal the use of long, slim flexures provides some isolation between the actuators. Nevertheless, parasitic thermal crosstalk may be observed with the use of a stroboscopic image capture system. In Figure 76, the device is actuated with ramp signals in the $X$ and $Y$ directions, with the intent of scanning a box-shaped path. The scan is performed at various voltage amplitudes, and the resulting position of a corner of the central shuttle is measured as a function of time.

Due to heat flow between the actuators, the pattern is distorted into a parallelogram. In addition, the square-law dependence of deflection on voltage is apparent in the data, since the distance between 
concentric parallelograms increases as a function of drive voltage. The temperature-based position measurement technique may be used to mitigate thermal crosstalk. The red inset box demarks the positioning range of the scanner if such a controller were used. Alternatively, the coupling coefficients between actuators can be extracted from this data and a coordinate transformation can be performed before generating the drive signals.

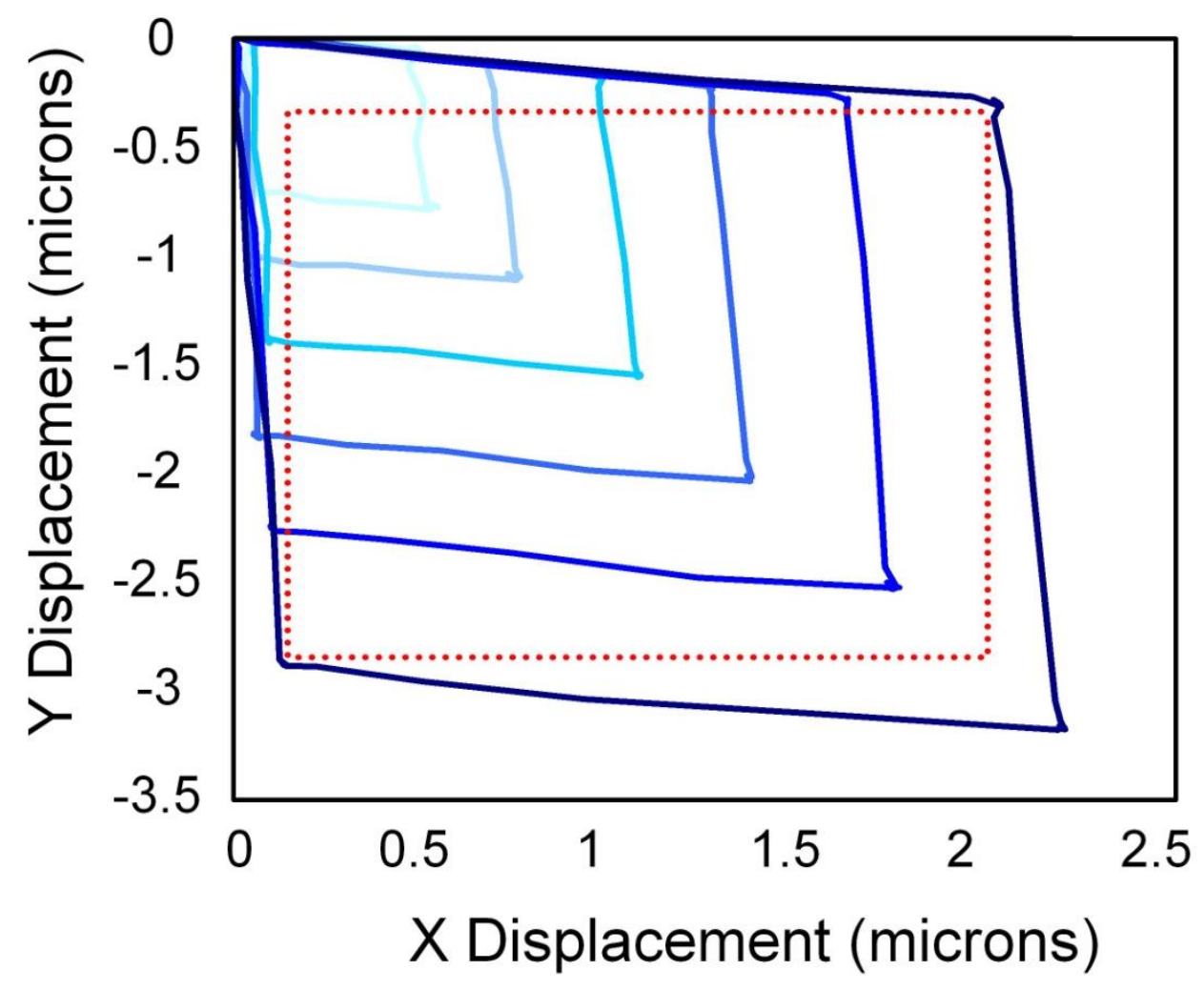

Figure 76: Measurement of Parasitic Coupling Between Lateral Actuators

Thermal coupling from the lateral actuators to the vertical actuator is more problematic, as it interferes directly with the tip-sample interaction measurement. Heat from the lateral actuators couples into the vertical actuator through the flexures, and there is no shunt path for the heat in this design. When the vertical actuator is not powered, the steady state temperature of the central shuttle assumes the average temperature of the lateral actuators. The coupling is detected as a shift in the measured resistance of the heating resistor of the Z-actuator. A controller was implemented to mitigate this effect, with results shown in Figure 77. A lateral scan voltage was applied to the X-actuator, while the beam that houses the self-heating resistor of the Z-actuator was imaged in an SEM. The geometry of the measurement is also shown in the Figure. 

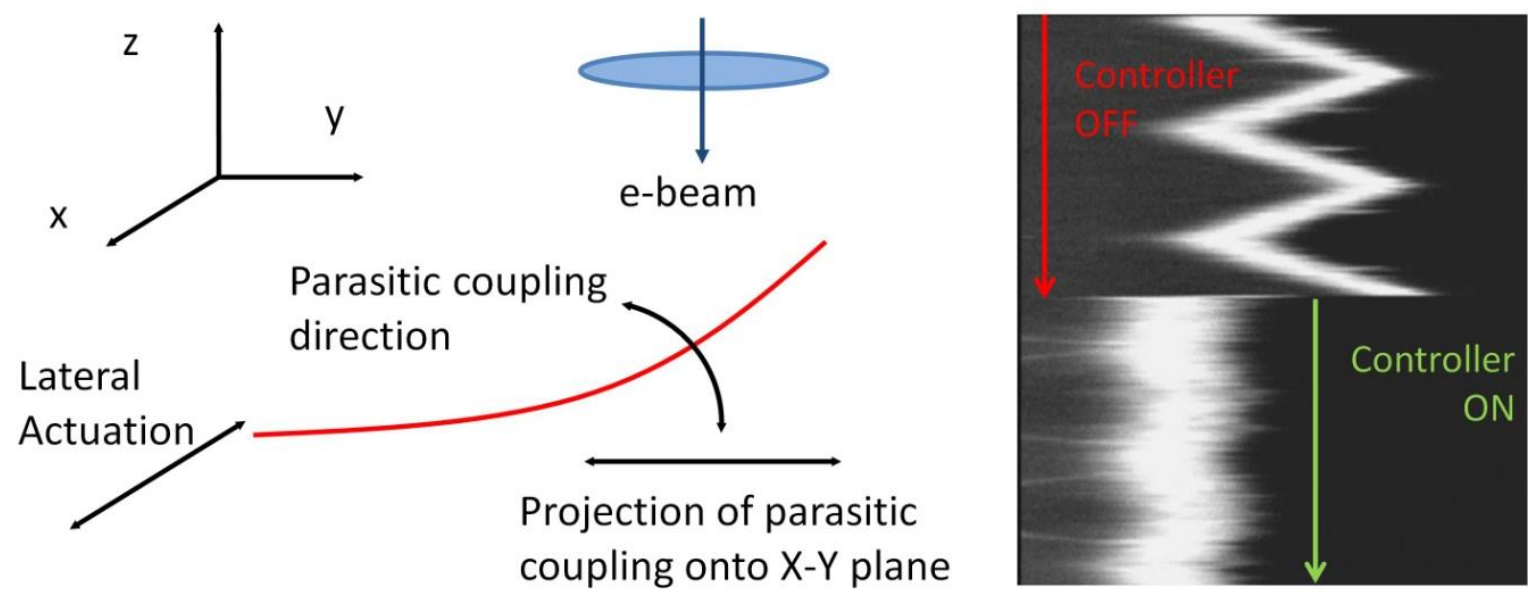

Figure 77: Temperature Coefficient of Resistivity-based Control of a Vertical Bimorph

The most severe manifestation of thermal cross-talk occurs between the vertical actuator and the piezoresistors, as was discussed in section 5.4.2, and presented in figure Figure 73. As previously described, the parasitical signal in this case arises from asymmetries in the path of heat flow from the cantilevers to the substrates.

The device was used to obtain images of standard AFM calibration samples (Figure 78). The images were post-processed in order to remove distortion caused by the lateral scanners, and to align the observed features. It is evident that the resolution of the instrument is limited by several factors.

The geometry of the piezoresistive cantilevers in this device results in lateral compliance that is approximately equal to the vertical compliance of the beams. During imaging, several "sticking" events were observed when the vertical actuator failed to track topology. Both samples contain large topological features in which the tip could be trapped. As the lateral scanners continued to deflect, strain in the beam reached a critical value after which the cantilever could snap out of contact laterally. The images obtained with this instrument consist in part of the artifacts due to lateral sticking.

The long flexures that are used in this design to mitigate thermal coupling are compliant in the vertical direction. During imaging experiments, it was observed that the entire shuttle would deflect when the vertical actuator engaged a sample. The compliance of the flexures detracts from the strain that is captured in the piezoresistors and reduces the overall sensitivity of the device. 
At high bridge powers, the piezoresistors generate enough heat to deflect the bimorphs in which they are integrated. As discussed in the SARC model, contact with the sample provides a cooling path and reduces the deflection of the bimorphs, leading to frequent tip crashes.

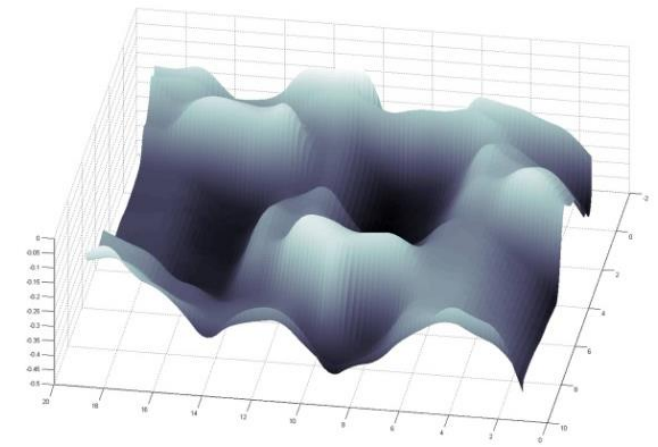

Image of 2D periodic grating obtained with single-chip AFM (3 micron period)

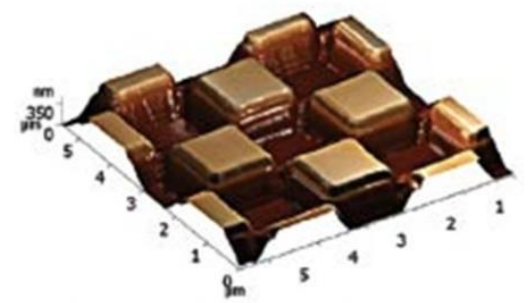

Image of 2D periodic grating obtained with conventional AFM

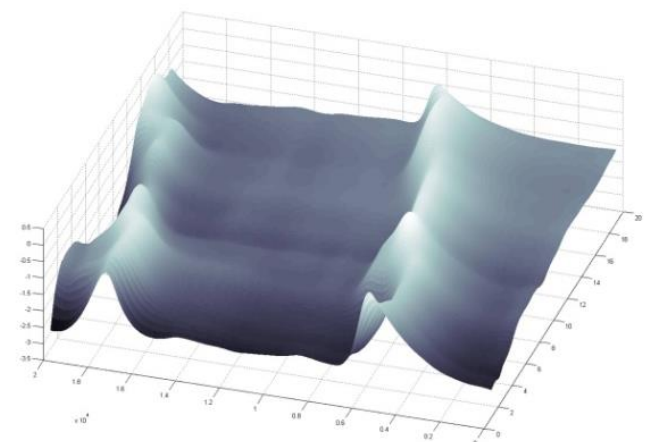

Image of triangular grating obtained with single-chip AFM (3 micron period)

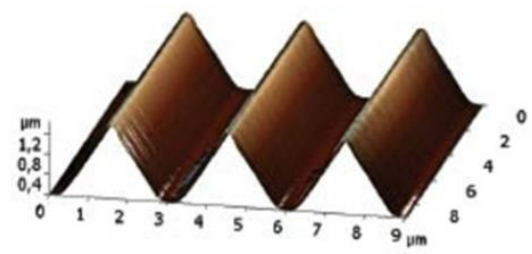

Image of triangular grating obtained with conventional AFM

\section{Figure 78: Contact Mode AFM Images}

Although its imaging performance is poor, this device served as a test bench to quantify the interplay of electrical, thermal and mechanical effects. It was determined that thermal coupling into the piezoresistors was the most significant source of disturbance. Also, the centrally located cantilever proved to be problematic when performing a coarse approach because the sample is in the line-of-sight of the microscope, and there is potential for interference with wirebonds. Several incremental improvements were in subsequent devices.

\subsection{Forced Oscillation and Higher Harmonic Detection}

As part of the experiments described above, intermittent contact waveforms were obtained, revealing the nonlinear nature of tip-sample interaction forces. The plots in Figure 79 represent the amplitude of the output signal under contact, intermittent contact, and non-contact conditions. All the signals in the chart are of comparable magnitude, indicating that the SNR of this device is poor. 


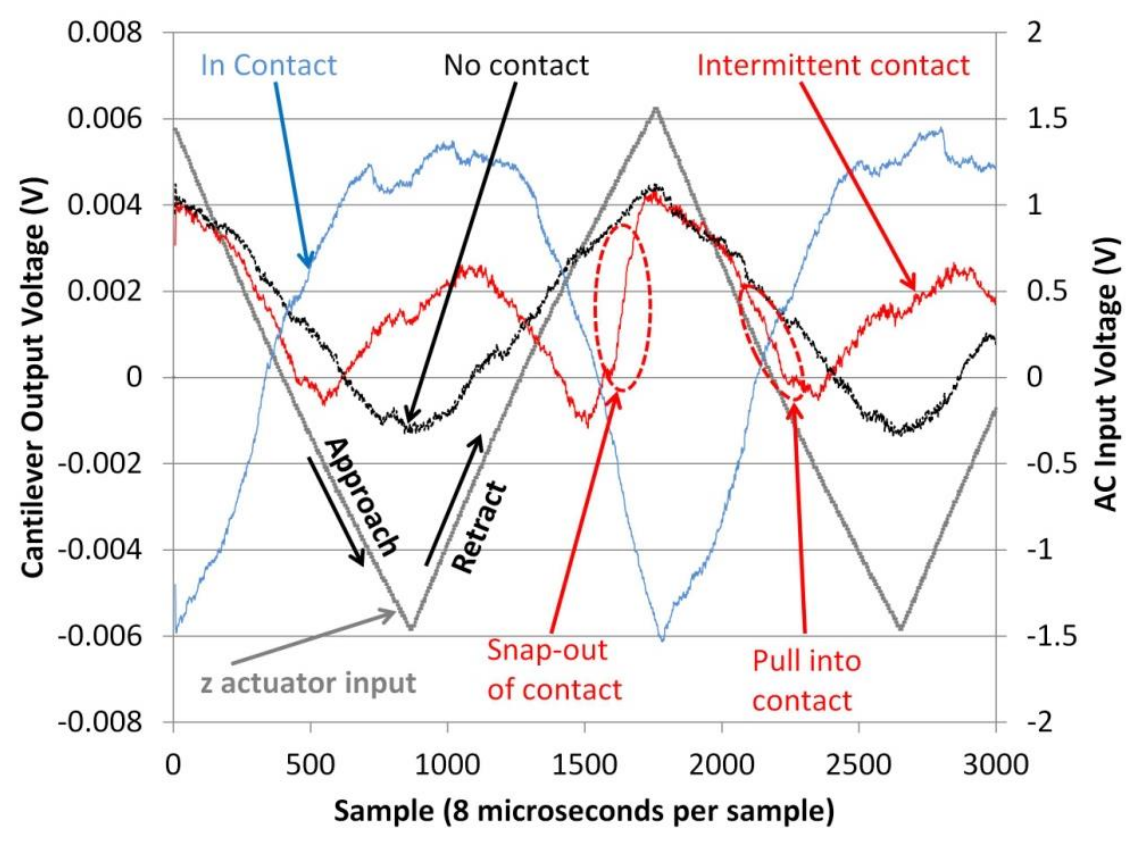

Figure 79: Data Obtained While the AFM is in Intermittent Contact with a Sample

The data was obtained at an actuation frequency of $70 \mathrm{~Hz}$ with a triangle wave input waveform. Three curves are used to represent various operating conditions:

1. The black curve represents the signal when the cantilever is close to the sample, but not in contact. Cooling of the sense cantilever dominates the output signal in this case.

2. The red curve represents the signal when the tip is in intermittent contact with the sample. Portions of the curve that diverge from the black curve represent interactions with the sample that are detected piezoresistively. During the retraction portion of the cycle, the tip snaps out of contact as it overcomes the attractive capillary forces. Pull-in is visible during the approach portion of the curve. When the tip is not in contact, tip-sample cooling results in the same curve as the non-contact case.

3. The blue curve represents the signal when the cantilever is in light contact with the sample at all times. During the approach, the piezoresistor signal increases in response to the increasing force exerted at the tip. The signal decreases during retraction. No pull-in or snap-out effects are observed, since the tip is always engaged.

With no sample present, the high frequency components of the triangle waveform are rounded by the RC circuit-like behaviour in the thermal domain. However, upon intermittent contact, the output signal 
clearly shows higher frequency content than the $70 \mathrm{~Hz}$ signal. When the tip is permanently engaged with the sample, the sharp peaks coincide with the peaks of the driving signal, but are slightly phase shifted due to the lag from the RC time constant. This peak obviously has higher frequency content as well, but it results from the driving signal and not the tip-sample interaction. In practise, if a sinusoidal drive signal is used, then the only high frequency contact would be from intermittent contact. Since the dominant parasitic thermal effects occur at the driving frequency, it is desirable to exploit this higher frequency content for imaging purposes, while suppressing thermal crosstalk.

Figure 80 is a system diagram of the method that is used to separate the tip-sample interaction component from the overall signal. A small-signal sinusoidal input with a DC offset $V$ is applied to the Zactuator. Heat from actuation couples to the piezoresistors, producing effects in the electrical (due to TCR) and mechanical (due to stress induced by the thermal bimorph effect) domains. The balanced cantilevers are biased with a DC voltage that is also applied to an off-chip trimming potentiometer. This balanced configuration rejects a fraction of the parasitic thermal coupling, but several asymmetries in the design remain and contribute thermal parasitics.

Time domain signal in intermittent contact

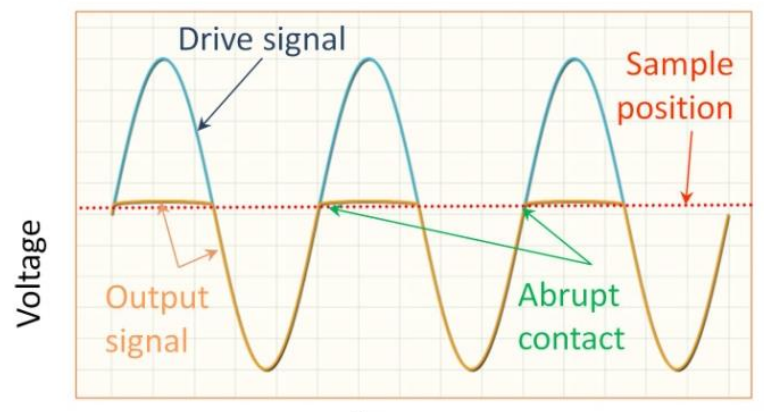

time
Morse Potential

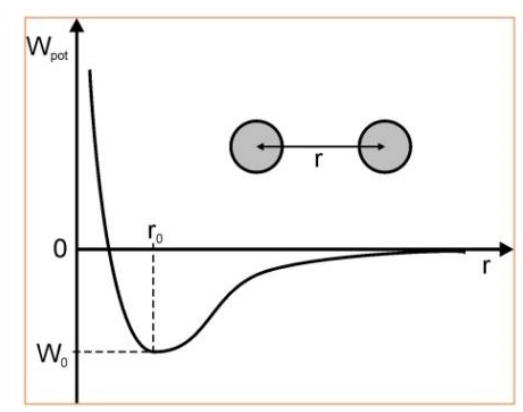

Balanced sensors

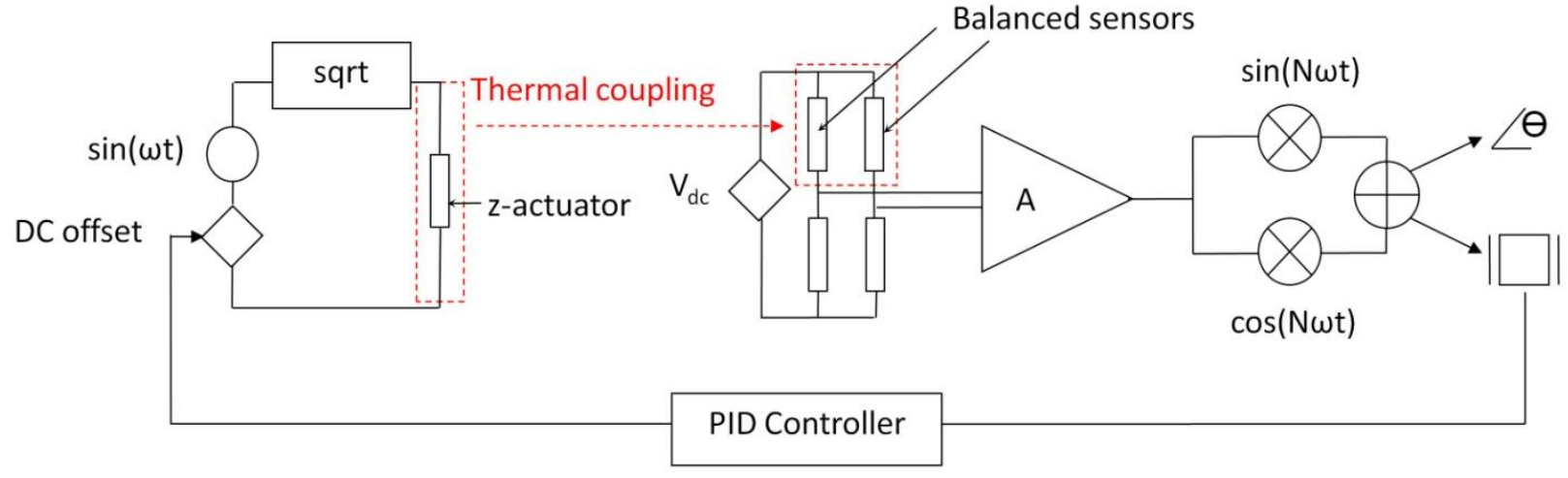

Figure 80: Higher Harmonic Detection Concept 
Consider the case in which the tip is in intermittent contact with the sample due to the periodic excitation of the Z-actuator. At the point of contact, abrupt changes occur in the mechanical and thermal boundary conditions at the tip, generating higher harmonic terms. An instrumentation amplifier is used to measure the signal from the bridge, and its output is demodulated using a pair of off-the-shelf modulator-demodulator IC's. The demodulation frequency is chosen at a harmonic that is above the thermal cutoff frequency. The harmonic power is held constant by adjusting the Z-offset voltage (hence tracking topology) while scanning laterally in $\mathrm{X}$ and $\mathrm{Y}$. The phase of the output signal may also reveal information about the sample topology; however, phase-based imaging has not yet been attempted.

This configuration rejects thermal parasitics from the TCR of the piezoresistors. In addition, a constant tip-sample separation is maintained during the scan, so the controller compensates for the coupling between the lateral and vertical actuators of the device. The result is an image which is tilted on a plane that represents the average temperature of the lateral scanners. This tilt is easily removed while scanning, with the use of a linear coordinate transformation.

One example of a device that is used in this mode is shown in Figure 81. The cantilever extends $\sim 300 \mu \mathrm{m}$ past the edge of the die, permitting observation of the tip-sample region while imaging.
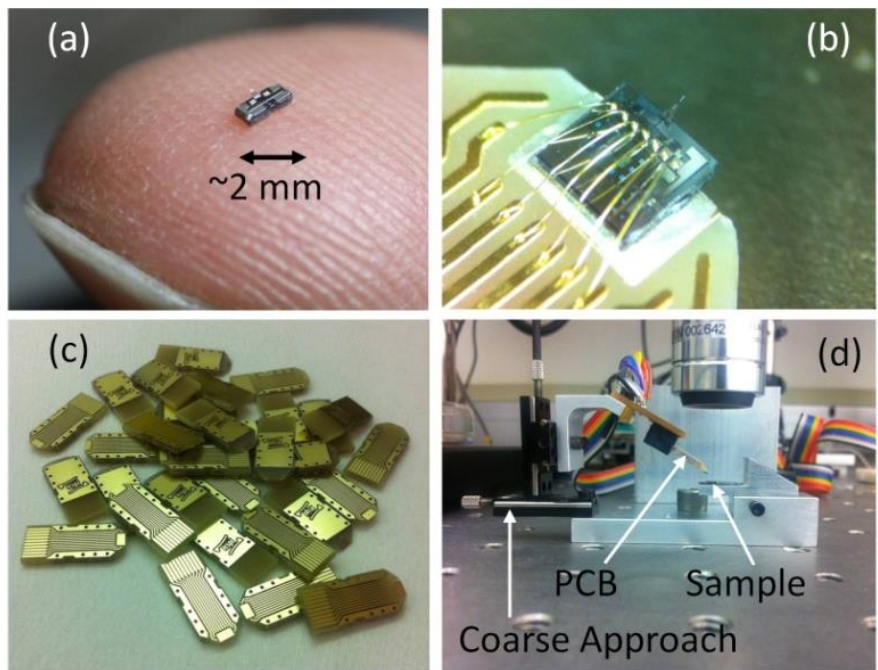

Figure 81: Packaging and Test Setup. a) released die after singulation, b) PCB carrier, c) packaged and wirebonded SPMs, d) Test Setup

To obtain this geometry, a laser-based trench formation process was developed. After the release, the chips are singulated by applying some bending strain near the regions where trenches are present. 
The coarse approach mechanism shown above is a manual micro-positioning stage, which was found to exhibit large amounts of drift. Several designs for automated stages have been developed over the course of this work, based on a number of transduction methods that are not discussed in this thesis.

The device geometry is shown in Figure 82. Some improvements over the contact-mode device are apparent. The balanced cantilevers are designed to be rigid in the lateral direction in order to overcome the lateral binding issue. Self-heating resistors for the vertical actuator are located $200 \mu \mathrm{m}$ away from the piezoresistors to reduce coupling and improve matching.

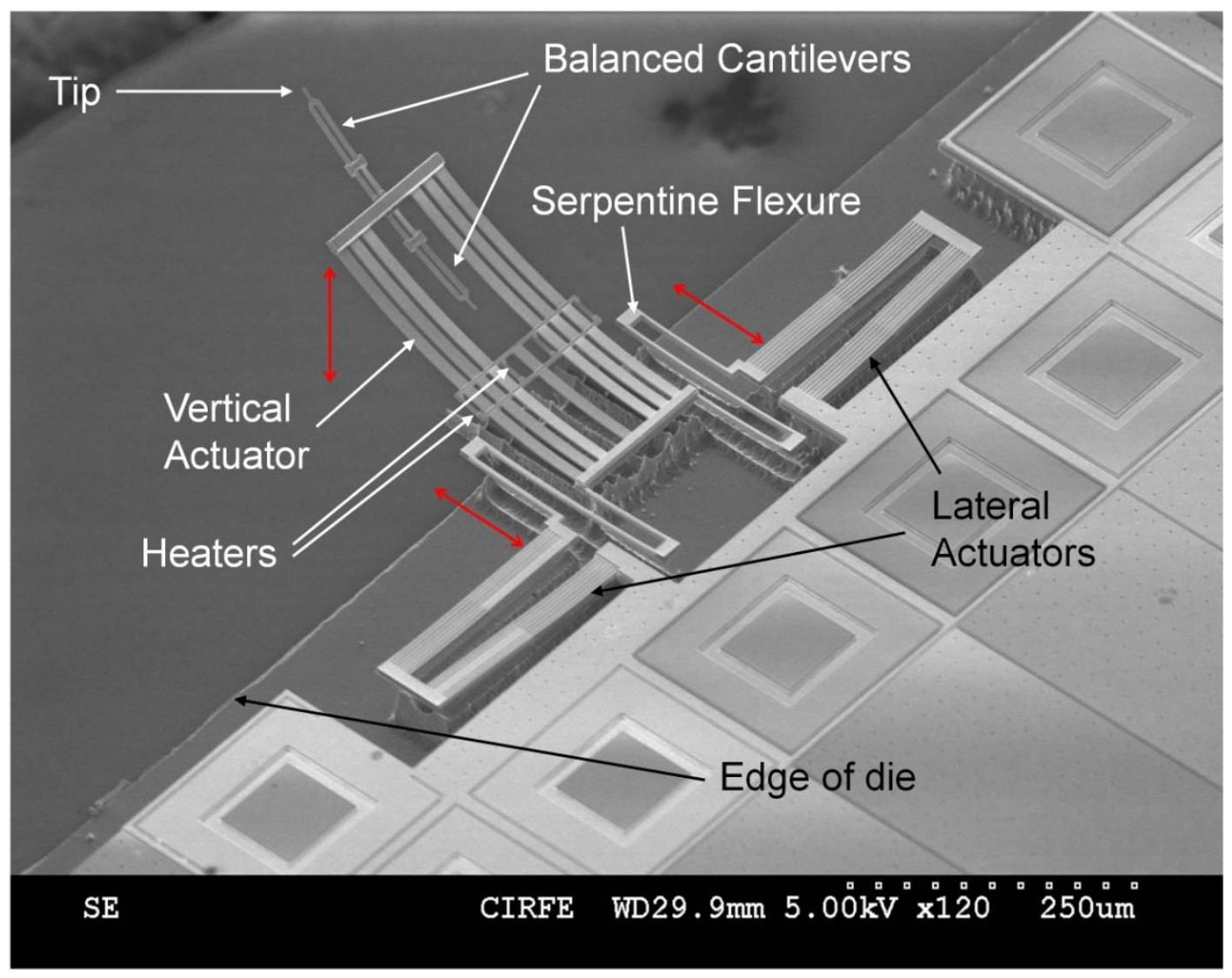

Figure 82: Cylindrical Coordinate Scanner

A feature of this geometry is that the average temperature during a lateral scan line can be held constant. This is achieved by applying a DC bias and a differential AC signal to the left and right actuators. A non-linear inversion is applied to the sum of the DC and AC signals in order to compensate for the $V^{2}$ dependence of the actuators' position and temperature. The result is a spherical coordinate scan $(r, \phi, \vartheta)$ in which the angle $\phi$ is related to the difference between the left and right actuator deflections, the radius $r$ is proportional to the average of the left and right actuator deflections and the 
elevation $\vartheta$ is provided by the out-of-plane bimorph. Since the total power in the lateral actuators is held constant during a line scan, coupling to the vertical actuator is eliminated. However, parasitic effects become evident upon actuation in the radial direction. The result is a tilting distortion of the image accompanied by limited vertical scan range.

When the device is actuated with no sample present, the majority of the signal from the sensors is at the forcing frequency. Upon intermittent contact with a sample, higher harmonics appear as shown in Figure 83.

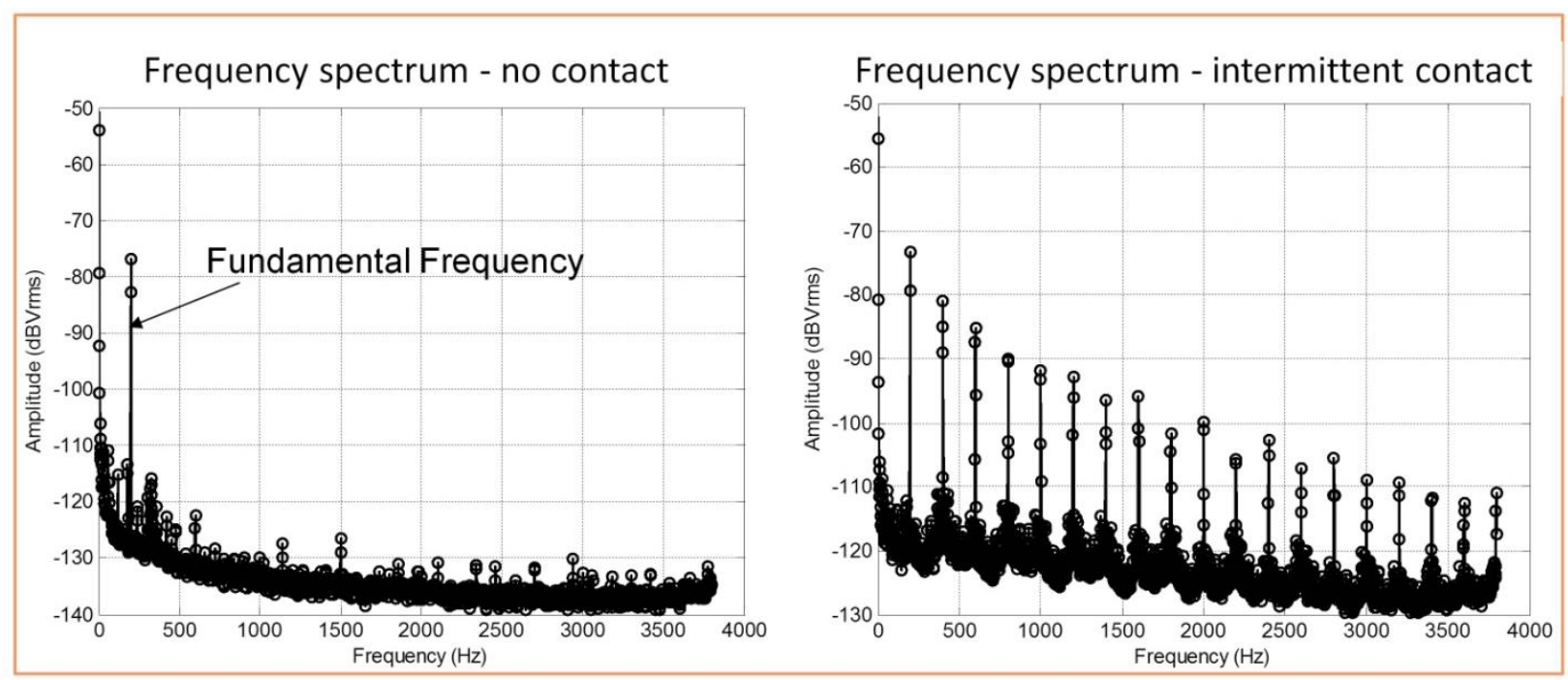

Figure 83: Detection of Higher Harmonics

Imaging of a triangular grating with a $3 \mu \mathrm{m}$ pitch and a $1.5 \mu \mathrm{m}$ height was performed with this device. No post processing was necessary, and the images in Figure 84 are screen captures of the UI.

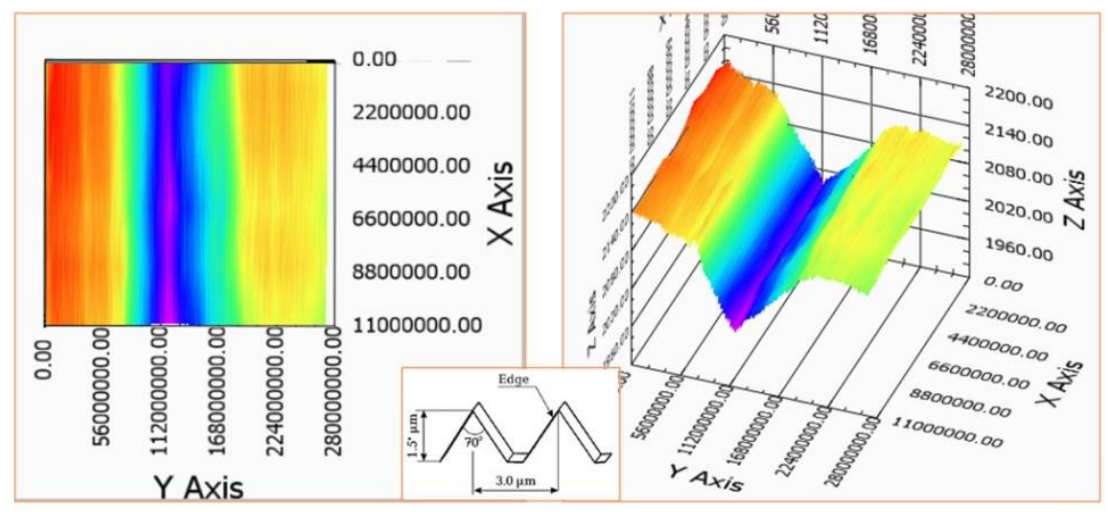

Top View
Sample Geometry

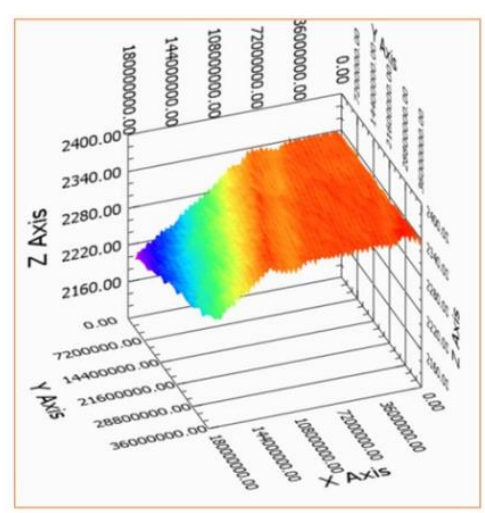

Small Scan Range

Figure 84: Images Captured at the Third Harmonic 
Higher harmonic imaging results with the cylindrical coordinate scanners show significant improvements over the contact mode images reported with the orthogonal scanners. However, the resolution is still poor in comparison with conventional SPM's. There are several drawbacks of this scanner design and mode of operation. In order to obtain an appreciable signal at a frequency beyond the thermal cutoff, the contact force is increased. This results in tip-wear that manifests as artifacts, such as the ridge that is visible in the small scan range image of Figure 84 . The serpentine flexure in the device experiences a larger moment from forces at the tip than the sensors, because it is located $\sim 300 \mu \mathrm{m}$ further back. Placing the piezoresistors near the base would guarantee that they experience the largest strain in the device; however, this would come at the expense of increased thermal coupling. The stiffness of the device should be tailored so that the maximum strain is located in the piezoresistors. In this design, the overall stiffness is much less than the stiffness of the EARC, resulting in pull-in that is observable during imaging.

\subsection{Summary}

In all of the microscopes discussed herein, balanced cantilever designs have been employed to generate a common-mode signal representing the thermal disturbances, and a differential signal that is intended to capture exclusively the tip-sample interaction. Unfortunately, asymmetries in the path of heat flow still exist due to the geometry of the system. When no sample is present, the heat flow from the sensors to the MEMS substrate is asymmetric. When a sample is present, the angle between the cantilever and the sample introduces a gradient in the temperature profile that is unrelated to the quantity being measured. These effects compromise the resolution of contact-mode AFMs.

A technique based on forced oscillation and higher harmonic detection was introduced to mitigate the thermal coupling issue observed in contact-AFMs. The method involves the measurement of a signal at a higher harmonic of the cantilever oscillation frequency that is generated by the nonlinearity of tipsample interaction. In order to achieve sufficient higher harmonic power for closed-loop imaging operation, the tip-sample contact force must be high, and this increases the tip-wear. Nevertheless, the images obtained in this mode of operation are an accurate portrayal of the topology of standard AFM calibration gratings. 


\section{Chapter 7. Dynamic AFM}

Non-contact AFM is by far the most widely used modality of scanning probe microscopy. Myriad variations on the operating principles have been studied to improve the resolution and bandwidth of the instruments, and to reveal material properties beyond topology. The common feature of all dynamic modes is that a resonant cantilever is used to provide some gain to the tip-sample interaction signal in the mechanical domain. In this chapter, two implementations of dynamic AFM are discussed: FM-AFM, and AM-AFM. These modes serve as the backbone of a large number of methods that are used in the SPM community to measure various types of tip-sample interactions.

\subsection{FM-AFM}

Dynamic AFM cantilevers can be stiffer than contact mode devices, since the flexural resonator improves force resolution due to its quality factor. The increased stiffness may improve the bandwidth of the device by augmenting its natural frequency. In addition, stiffer cantilevers are more stable in close proximity to samples, since they are less likely to experience pull-in from attractive forces. When imaging in the attractive regime with a negative force gradient, the tip-sample interaction may be modeled to first order as a spring in series with the cantilever constant. This has the effect of reducing the natural frequency of the resonance. Although it is possible to image in the repulsive region as well, it is not the preferred method in part because of the increase tip wear and the increased likelihood of tip crashes.

Figure 85 is a layout capture of the FM-AFM device, and Figure 86 shows SEM images of the released device before singulation. The device shown here has shorter piezoresistors than its contact-mode counterpart to ensure a reasonably high resonant frequency. A cantilever mass is included in order to separate the natural frequency of the flexural resonators from the natural frequency of the vertical actuator. Only one of the strain sensors is loaded with a tip-mass, while the other serves as a balance resistor that remains stationary during imaging. Thermal shunt paths provide a route for heat current to escape from the vertical actuators, effectively reducing operating temperature and thermal cross-talk. The cylindrical coordinate scanner geometry is chosen due to its capability to perform a line-scan at a constant temperature, its compact footprint, and the favorable geometry allowing for suspended cantilevers over the die edge. The stiffness of the device is enhanced through the use of shorter serpentine flexures and through the additional mechanical support provided by the thermal shunt paths. The device geometry reflects an overall stiffness budget of $10 \mathrm{~N} / \mathrm{m}$ referred to the tip which helps 


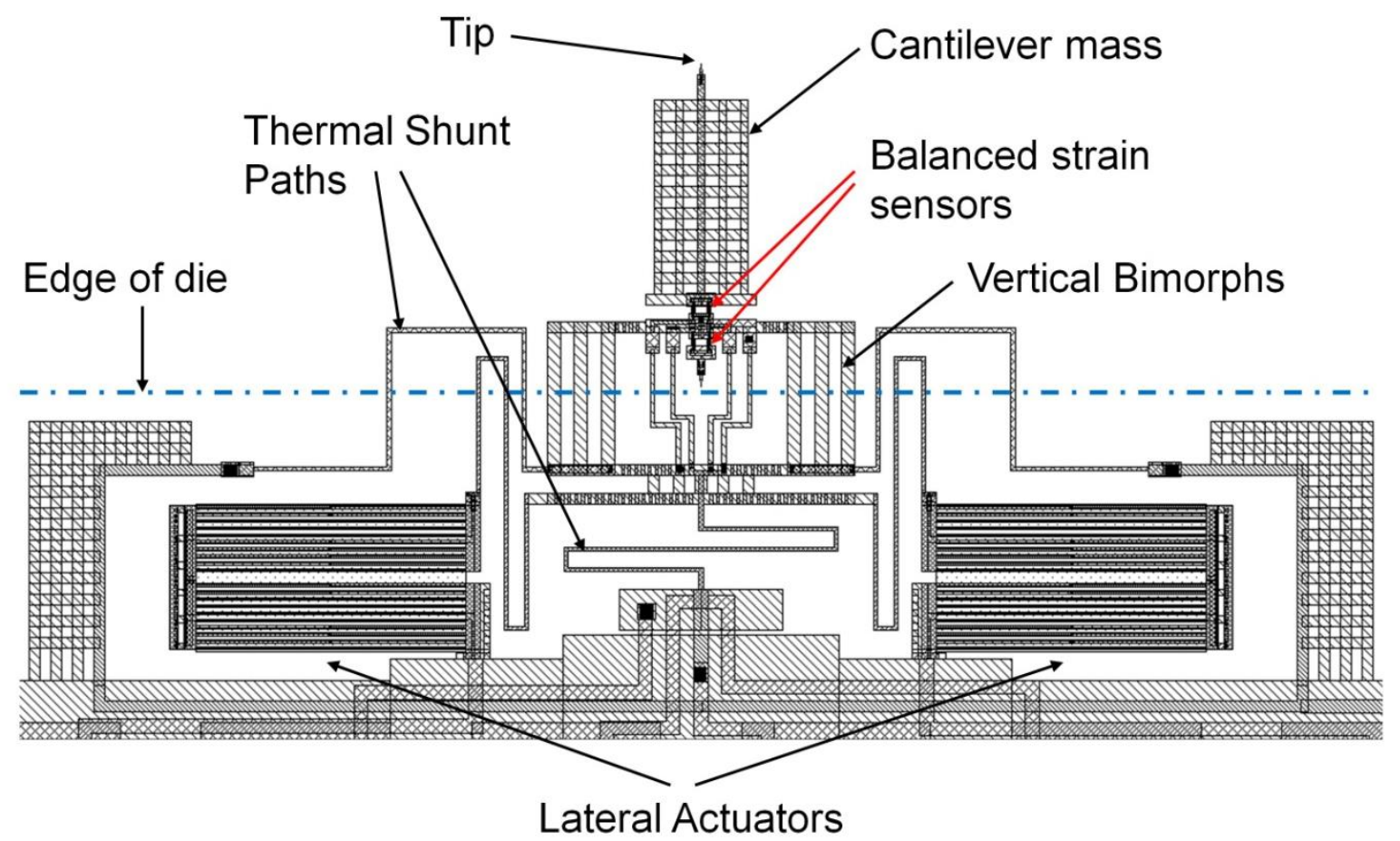

Figure 85: Dynamic AFM Device Layout
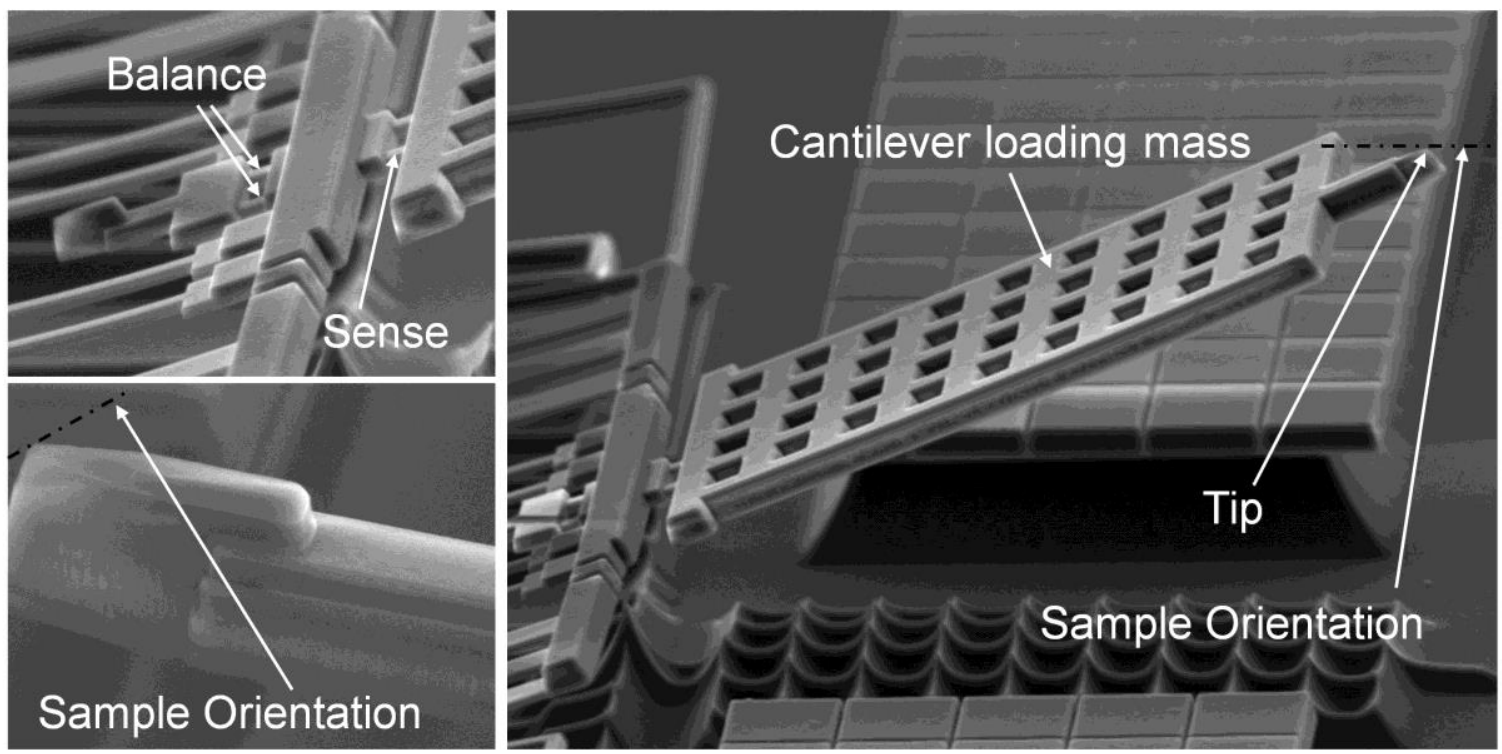

Figure 86: Close-up of Dynamic AFM Cantilever and Balanced Piezoresistors

to localize the strain from tip-sample interaction to the piezoresistors. The tip terminates in a wedgeshaped geometry that violates foundry design rules, but consistently results in a radius of curvature 
below $75 \mathrm{~nm}$ as confirmed by test structures. This radius corresponds to the location and orientation of the tip-sample region as indicated in Figure 86.

Imaging in FM-AFM mode was performed with in the configuration shown in Figure 87 below. The driving scheme corresponds to the EARC method. In general, high resolution FM-AFM measurements have been relegated to vacuum conditions, where the Q-factor may be enhanced to values of $\sim 10,000$ for conventional cantilevers. Under these conditions, the principle advantage of FM-AFM is that the bandwidth is not compromised by the Q-factor, since a PLL can be used to track the phase. For sufficiently flat samples, the phase information may be used to control the amplitude of the AC signal, improving bandwidth further. Atomic resolution imaging has been performed with conventional AFMs under these conditions.

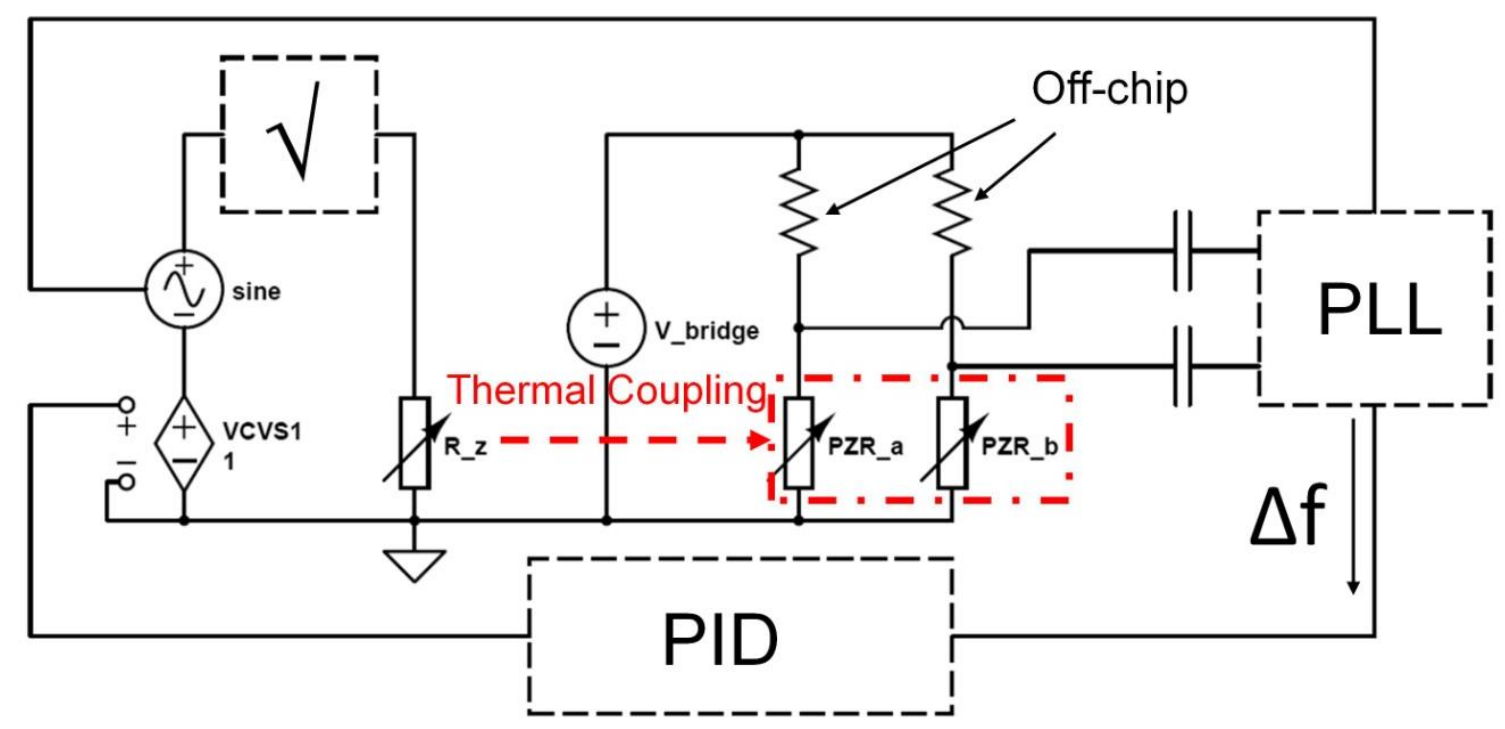

Figure 87: FM-AFM Configuration

The pressure dependence of $Q$ in CMOS-MEMS cantilevers was measured in a vacuum probe station. Figure 88 demonstrates an increase in Q-factor from $\sim 100$ in air to 1000 in a vacuum pressure of 0.02 mBar. These results do not make use of the Q-pumping method reported earlier.

Images of AFM calibration gratings and vias on a CMOS chip were acquired using the FM-AFM mode. The sample topology is on the order of $1.5 \mu \mathrm{m}$, so the PID output was used to control the DC-offset applied to the vertical actuator. 


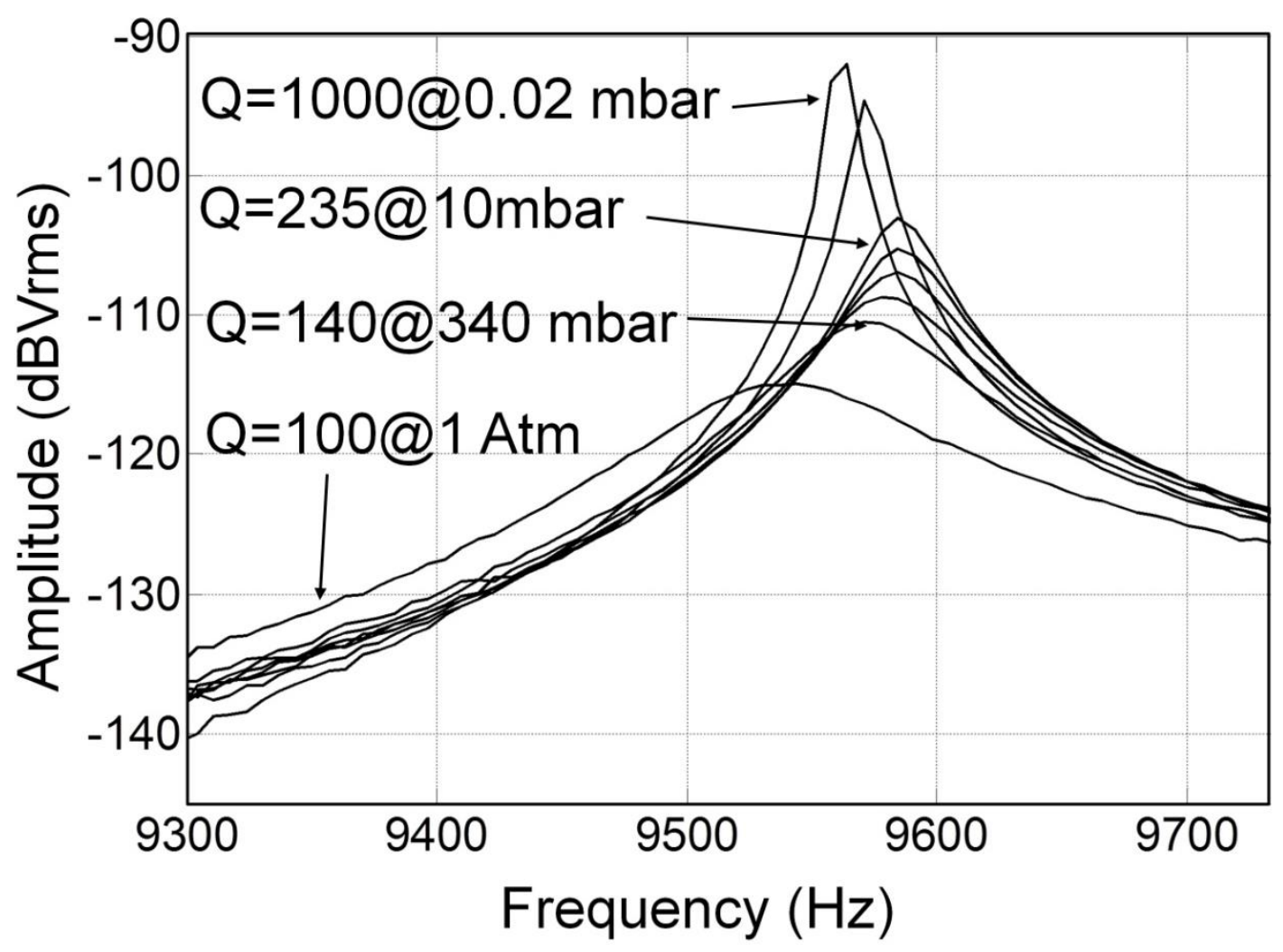

Figure 88: Q-Factor and Resonant Frequency of Dynamic AFM as a Function of Pressure

The bandwidth and resolution obtained with FM-AFM exceeds what was demonstrated with contact and intermittent contact modes. It was observed that the frequency shift from tip-sample interaction is sensitive enough to quantify vertical drift in the coarse approach mechanism in time scales of $\sim 10$ seconds, which suggests that an improved coarse approach mechanism is needed for high resolution imaging. Several pull-in events can be seen in the FM-AFM images, mostly resulting from abrupt variations in the sample height. In several instances, the simple PID controller was not able to recover from pull-in events due to the reversal of the slope of force gradient, and hence the reversal of the frequency shift. In these cases, the frequency of the PLL shifts up to its maximum value where it remains until the user intervenes. Changing the polarity of the PID gain momentarily allows normal operation to resume. A more sophisticated controller that can adapt to repulsive or attractive conditions would improve the robustness of the device to pull-in effects. A better alternative may be to use a stiffer device. 


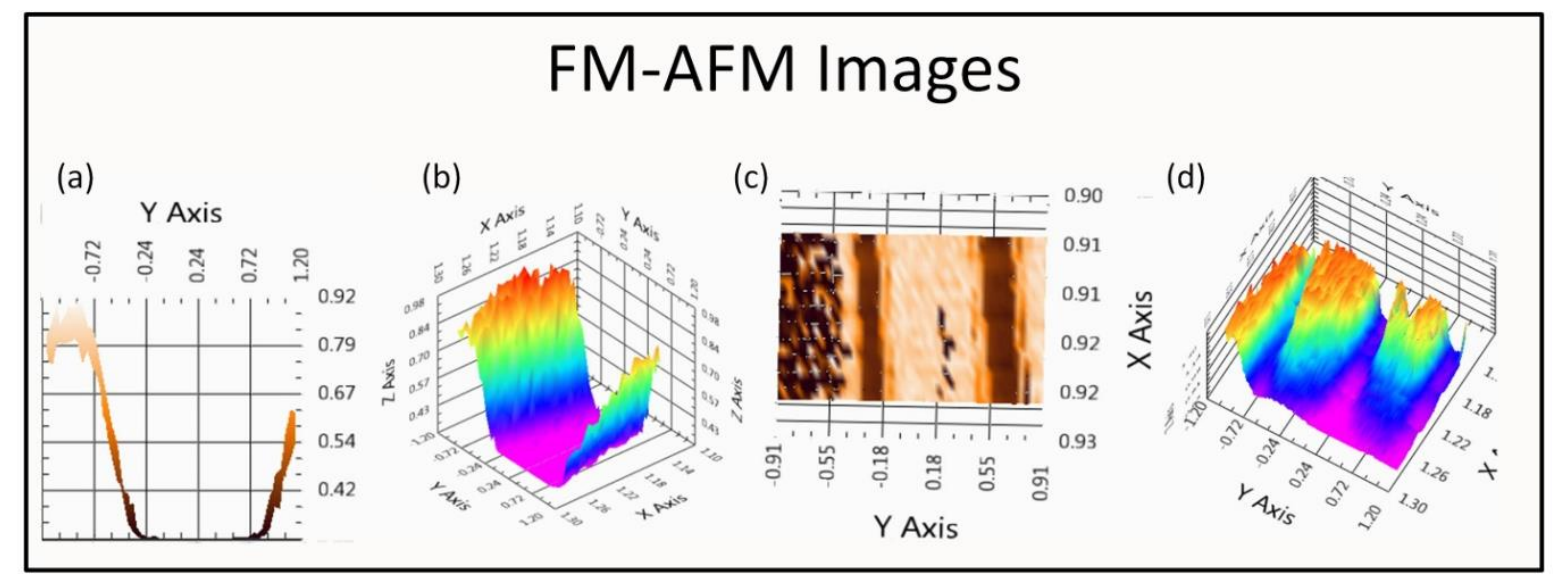

Figure 89: FM-AFM Images of a calibration grating (left) and Exposed Vias on a CMOS-MEMS chip

The natural frequency of the resonant cantilevers is influenced by temperature, presumably due to a combination of the temperature coefficient of Young's Modulus, and the geometric changes experienced by bimorphs at elevated temperatures. Over a $2 \mathrm{~V}$ range of Z-actuation, the frequency shifts down by $15 \mathrm{~Hz}$. Thus, the PID is configured with a setpoint of $-30 \mathrm{~Hz}$, so that tip-sample interactions dominate over the signal from heating. The temperature coefficient of resonance may contribute to the instabilities that were observed during FM-AFM operation.

\subsection{AM-AFM}

The most widely used mode of SPM is AM-AFM. The cantilever is excited at a frequency slightly above its natural frequency, so that the amplitude of oscillation increases when attractive tip-sample interaction forces shift the resonant frequency upwards. A PID is used to adjust the height of the cantilever in order to maintain a constant oscillation amplitude while imaging. Although a high quality factor improves the MDF of the instrument, it also reduces the imaging bandwidth. When a resonator encounters an impulse in its driving force or load impedance, ringing occurs for a length of time that is proportional to its $Q$ value. It is this settling time that limits the achievable imaging bandwidth.

Several devices were used to image samples using the AM-AFM mode, including the device that was used in FM-AFM measurements. Figure 90 below shows another device with a similar design pattern. Images of rectangular gratings with $90 \mathrm{~nm}$ step heights were recorded at a line-scan speed of $3 \mathrm{~Hz}$. This imaging mode was observed to be more robust than FM-AFM, as evidenced by continuous imaging without tip crashes or pull-in over a time frame of 30 minutes. In addition, the sensitivity of the resonant amplitude to vertical actuation is lower than the frequency sensitivity of the FM-AFM to the Zactuator offset. 


\section{AM-AFM Images}

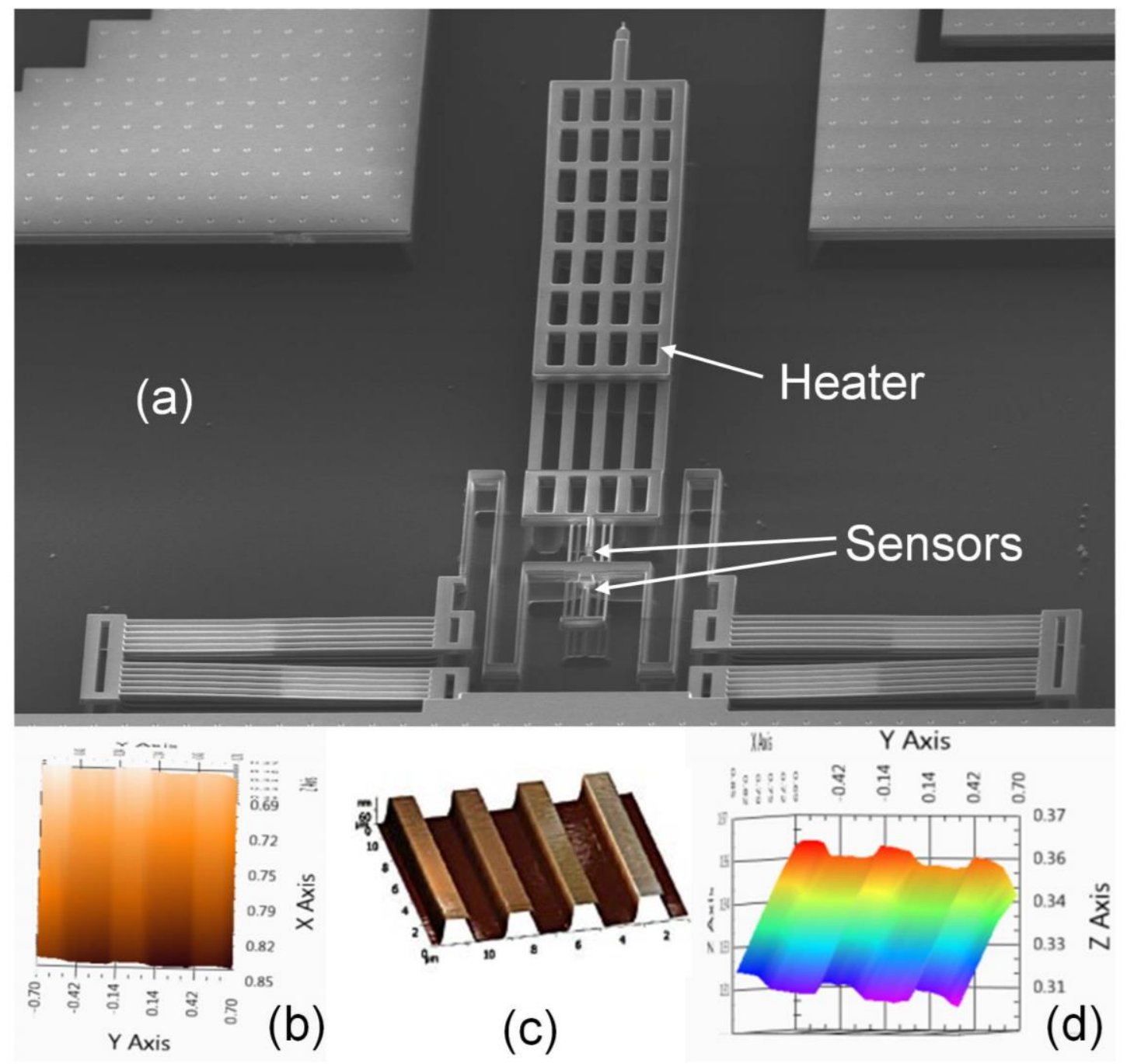

Figure 90: AM-AFM Images of a 90nm AFM Calibration Grating

\subsection{Summary}

To summarize, several devices have been used as dynamic AFMs. FM-AFM devices with moderate Qfactors were used to image samples under ambient conditions. The performance of these devices was superior to that of contact-mode AFMs, in part because of enhanced stiffness and the gain from resonant operation. AM-AFM devices were also used under ambient conditions, with further improved results. 90nm patterns on a Silicon AFM calibration grating were imaged for several hours at various scan speeds. 
Oscillation of the tip at its natural frequency was used to significantly improve the tip-sample interaction signal. The sensitivity of the natural frequency to thermal coupling is much lower than the offsets in the DC output of piezoresistors that experience oscillating temperatures. In addition, the resonant frequencies of the cantilevers are chosen to be beyond the thermal cut-off frequencies of vertical actuators. Under these conditions, a second order effect limits the performance of the instrument: the cantilever height is modulated by the average temperature of the lateral actuators, through heat transfer to the vertical actuator that is used to track topology. This introduces distortions in the image, which may be removed through a coordinate transformation. It also limits the area of the scanned region. A band-aid solution may be to mount the sample at an angle with respect to the single-chip SPM.

The recent Q-enhancement results reported in section 4.3 suggest that it may be possible to perform high bandwidth, high resolution imaging in the FM-AFM mode in ambient conditions. UHV conditions are necessary to maintain pristine surfaces, especially for materials like Silicon, which grows a native oxide almost instantly upon exposure to atmospheric conditions. However, there is a wide range of FMAFM applications for which operation under ambient conditions would be most welcome. 


\section{Chapter 8. Scanning Thermal Microsopy}

SThM is a type of SPM that can map the local temperature and thermal conductivity of a surface with nanometer scale resolution. Thermal measurements at these length scales are of both scientific and industrial interest. Vendors of SThM-capable instruments have published application notes ranging from the detection of phase change temperatures in polymer blends, to the subsurface imaging of faults in CMOS devices. The Millipede is an early example of the use of a parallel array heated probes to read and write features in a medium to store data. In [183], the authors discuss the use of SThM to pattern nanometer scale features, highlighting the high resolution and the versatility of this approach. High resolution is obtained by spatial thresholding, which ensures that only a small region of the surface is hot enough for a thermochemical reaction to proceed. The versatility of the approach stems from the fact that a wide range of materials can be processed.

Two types of probes may be used for SThM: probes based on thermocouples, and probes based on bolometers. In this work, single-chip SThMs have been designed with both types of probes. The thermocouples are based on the Seebeck effect as discussed in section 3.6, and the resistive temperature sensors (bolometers) are the same as those used for position sensing in electrothermal actuators.

In this chapter, the design of both types of single-chip SThM's is first introduced. Tip-sample approach curves are then presented and explained by a model of tip-sample interaction in the device. Imaging results of a silicon surface are then presented. Finally, results of patterning experiments are presented.

\subsection{Device Design}

In the design process of single-chip SThMs, effort should be made to improve the device's sensitivity to thermal interactions at the tip-sample interface. The designs for bolometer and thermocouple-based devices are shown in Figure 91 below. The SThM design leverages several of the features that have been discussed thus far. Tips are thermally isolated using the structures discussed in section 3.4. Thermal isolation is intended to localize the heat transfer to a region around the tip-sample interface while rejecting thermal coupling to the rest of the device. A balanced pair of temperature sensors is used to further suppress the parasitic thermal coupling. The common mode signal generated by the sensors is rejected in the electronics, which improves the dynamic range of the instrument. The heater resistor for the vertical actuator is at its base, so that at high actuation frequencies the AC temperature variation is attenuated over the length of the beam. 

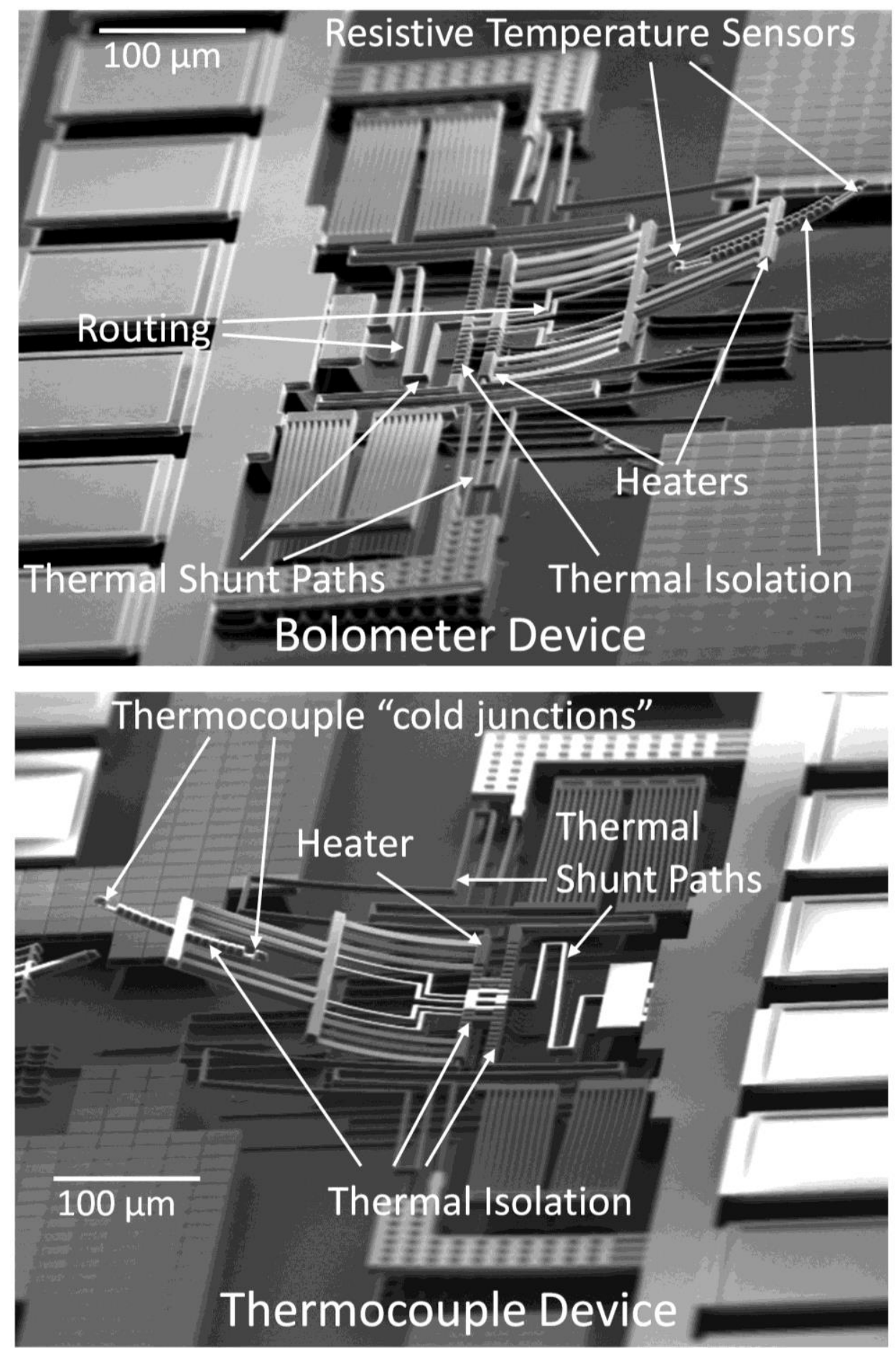

Figure 91: SThM Device Geometry

The layouts of the two devices are quite similar. Other than the layout of the temperature sensor, the only difference between the devices is that the bolometer device requires 2 electrical contacts while the thermocouple device only requires 1 . The other contact to the thermocouple is routed through the 
polysilicon material in order to ensure that there is a temperature gradient between the junctions. The thermocouple enjoys improved thermal isolation from the remainder of the device.

Another noteworthy feature in the design is the use of non-conductive thermal isolation paths between the vertical actuator and the remainder of the device. As discussed in section 3.4.2, the thermal conductivity of $\mathrm{SiO}_{2}$ is over $100 \mathrm{X}$ lower than that of the metals. A shunt path is directly connected to the heaters of the vertical actuator, reducing their sensitivity to thermal coupling and their efficiency, and improving their closed-loop position resolution. Because these isolation paths are non-conductive, the signals from the temperature sensors are routed through the thin beams and the thermal shunt path at the base of the device. The routing is designed so that the series resistance to the sensors is matched.

In both designs, there is an additional heater placed at the base of the isolation paths leading to the temperature sensors. This resistor is used to modulate the temperature of the sensors, such that lock-in techniques may be used. The auxiliary heater is particularly useful in the thermocouple device, since it heats up one of the junctions in each thermocouple. The base junction is therefore referred to as the "hot" junction, and the "cold" junction measures heat transfer to the sample.

\subsection{Tip-Sample Approach Curves}

Tip-sample interaction curves were obtained with both devices in order to validate the model and confirm that the device can measure heat transfer as intended. The curve shown in Figure 92 was obtained using a thermocouple device. Measurements of the output power at the fundamental, second harmonic, and third harmonic frequencies are shown.

The curve was obtained while applying a $50 \mathrm{~Hz}$ sinusoidal waveform to the auxiliary heater, thereby modulating the hot junction temperature of the thermocouple, as well as its separation from the sample. The vertical actuator was used to retract the tip from the sample with an applied offset voltage. A simplified version of the model from section 3.6.5 may be used to interpret these results. Since $50 \mathrm{~Hz}$ is well below the thermal time constant of the thermal isolation path, a lumped model in the thermal domain suffices. The model is shown in Figure 93 below. The following discussion provides a heuristic interpretation of the measurements, in which component values are neglected. 


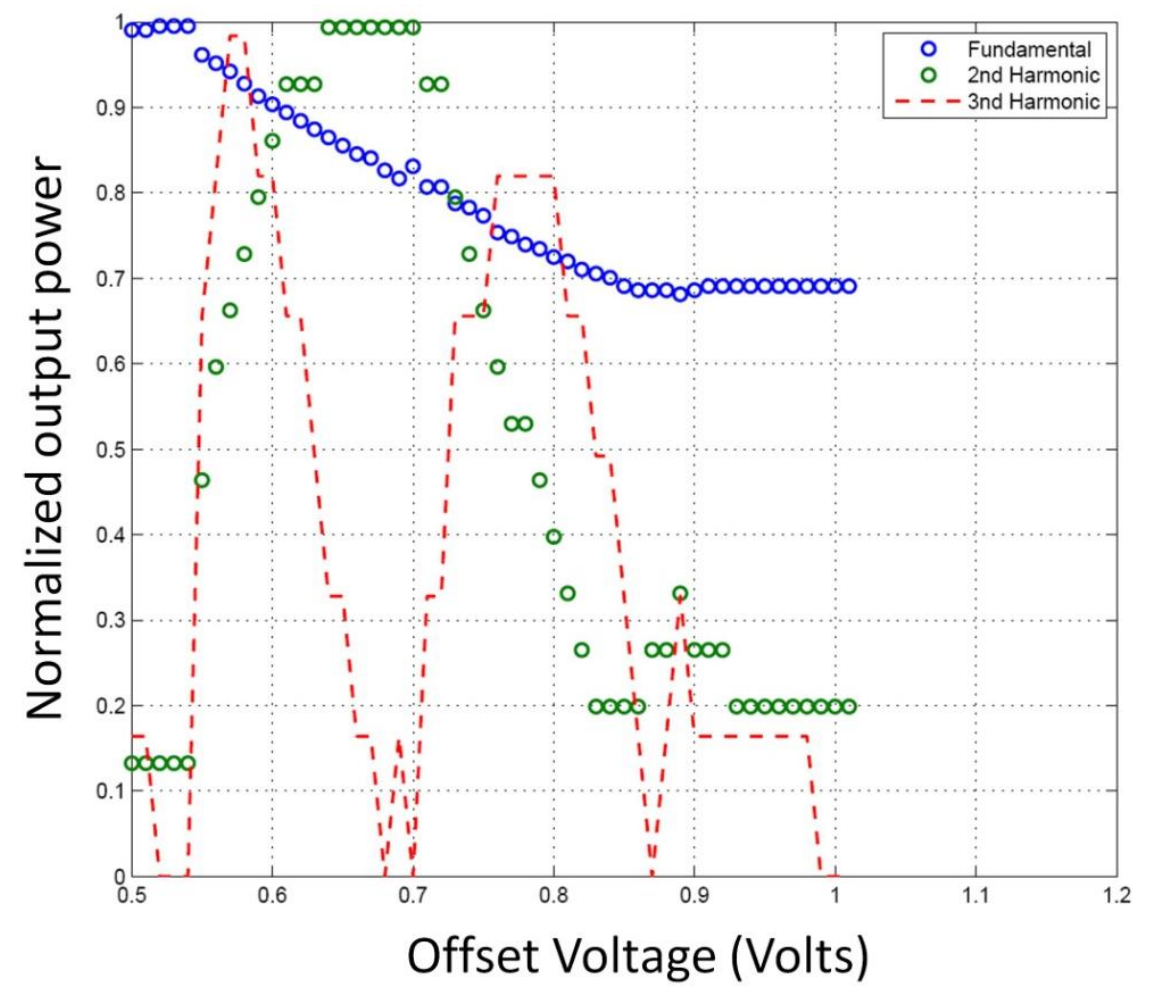

Figure 92: SThM Tip-Sample Approach Measurements

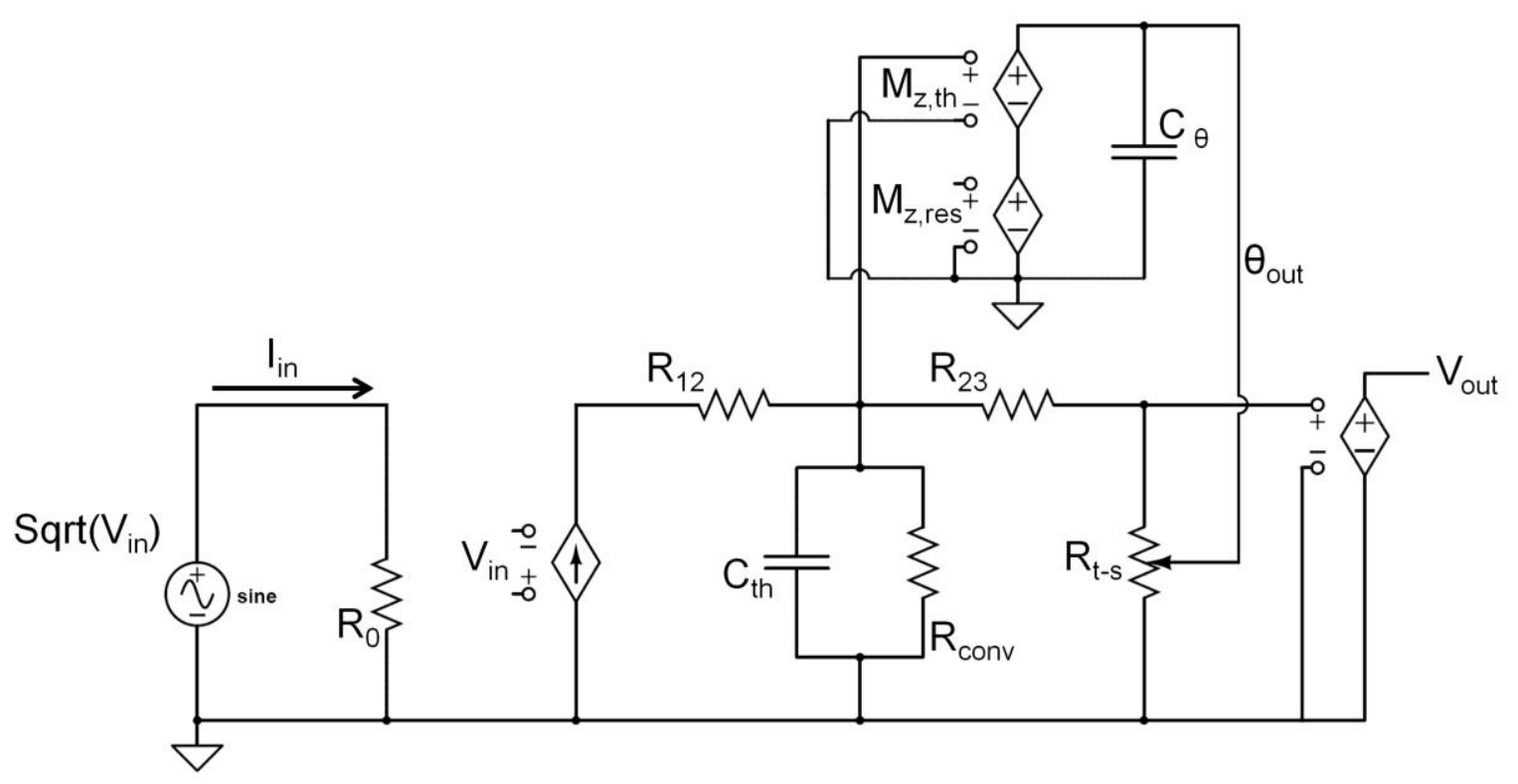

Figure 93: Tip-Sample Interaction Model for SThM

The temperature of the tip is a function of the heat current produced through Joule heating, as well as heat loss through the tip-sample gap. In this experiment, the square root circuit was used to linearize the temperature of the auxiliary heater. Thus, the thermal current source can be represented simply as: 


$$
Q=\left(\sqrt{V_{d c}+V_{a c}}\right)^{2}=V_{d c}+V_{a c}
$$

The tip sample gap is a linear function of the temperature:

$$
d_{t-s}=K\left(V_{d c}+V_{a c}\right)
$$

The resistance of the tip-sample gap is:

$$
R_{t-s}=\frac{d_{t-s}}{\kappa_{\text {air }} A_{t i p}}
$$

Where $\kappa_{\text {air }}$ is the thermal conductivity of the air gap and $A_{\text {tip }}$ is the surface area of the tip. Ohm's law is used to calculate the tip temperature:

$$
V=I R \therefore \Delta T=Q R_{t-s}=\frac{K\left(V_{d c}+V_{a c}\right)^{2}}{\kappa_{a i r} A_{t i p}}
$$

The temperature can therefore be expressed in the time domain as:

$$
\Delta T=\frac{K}{2 \kappa_{a i r} A_{t i p}}\left(2 V_{d c}{ }^{2}+V_{a c}{ }^{2}+4 V_{d c} V_{a c} \sin (t)-V_{a c}{ }^{2} \cos (2 t)\right)
$$

For simplicity, it can be assumed that the sample position corresponds to a dc bias of OV. At high DC bias voltages, the dominant term will be at the fundamental frequency. This corresponds to the situation when the tip is far from the sample. When $V_{d c}=V_{a c}$, the tip is at the threshold of contact. At this point, the amplitude of the fundamental is 4 times higher than the second harmonic amplitude. At values below this, the tip comes into intermittent contact with the sample, so the temperature will change abruptly, giving rise to higher harmonics. The time domain waveforms corresponding to these situations are shown below in Figure 94.

A diode is added to the model to account for the nonlinearity that occurs upon tip-sample interaction. The diode clips the output position at the point of contact, where the tip-sample resistance is at a minimum. When $V_{d c}$ drops below $V_{a c}$ the clipping becomes apparent as in Figure 95 which depicts the position of the tip as a function of time. When the tip is in contact, heat flows into the sample and causes an abrupt, large temperature change that adds power to the $2 \mathrm{f}$ and $3 \mathrm{f}$ signals. Once the tip is in full contact with the sample, the temperature of the sensor drops to that of the sample. Thus, there is a large temperature difference between the hot junction and the cold junction, which explains the 
increase in power at the fundamental frequency. Since the tip-sample resistance is no longer a function of position, the second harmonic vanishes. Finally, since there are no abrupt changes in temperature, the third harmonic and higher harmonics are no longer present either.

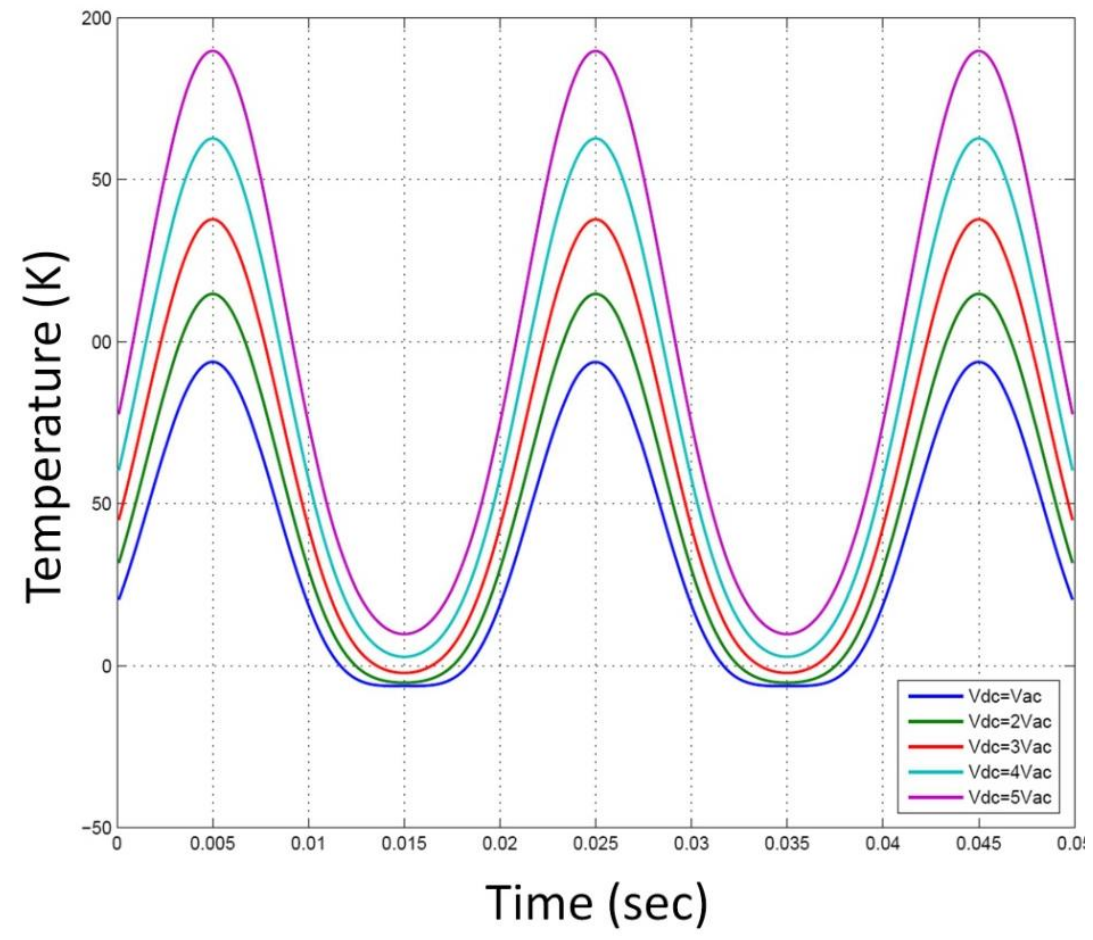

Figure 94: Time-Domain Temperature of SThM During Tip-Sample Approach
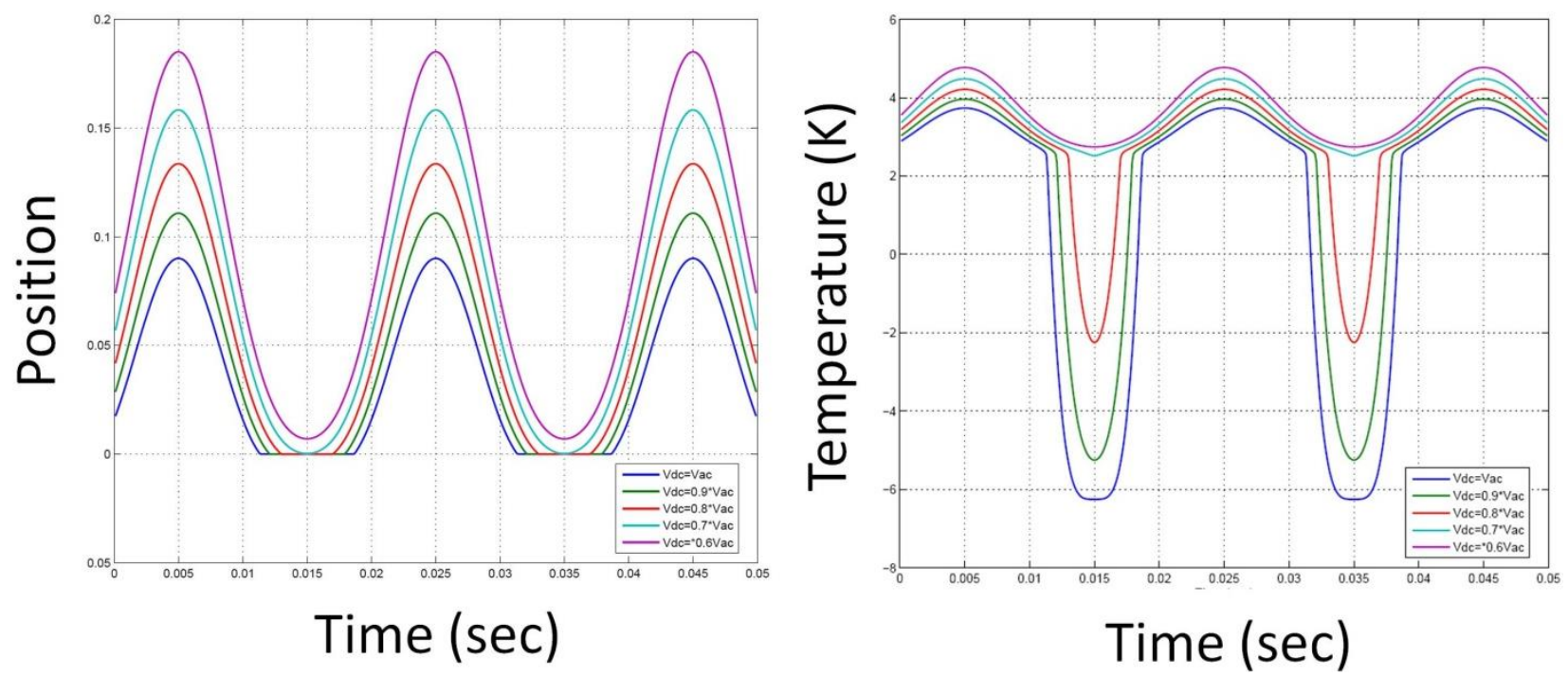

Figure 95: SThM Tip-Sample Approach. Left: Position of tip, Right: Temperature of tip. 


\subsection{Imaging results}

The thermocouple-based SThM was used to image the substrate of a CMOS-MEMS chip. The sample contained several topological features that could be detected by the instrument. Due to the fact that silicon has a high thermal diffusivity, abrupt contact causes a large temperature change which is easily measured. Imaging was performed while holding the power constant at the second harmonic. The first image obtained using this device is shown below in Figure 96.

The feature that was imaged is the shadow of a previous SThM device that is imprinted into the substrate during the sacrificial Si etch step of the release process. During imaging, it was observed that the phase of the first and second harmonic were not constant while their amplitudes were held constant by a PID controller. When imaging transitions between metal and silicon dioxide, a significant phase shift was observed. According to the thermal model of a thermal isolation structure, the thermal impedance at the tip-sample interface may alter the phase of the $A C$ temperature at the thermocouple junction. This would suggest that it may be possible to obtain thermal capacitance images of the sample under test; however, imaging using the phase information has not yet been performed.
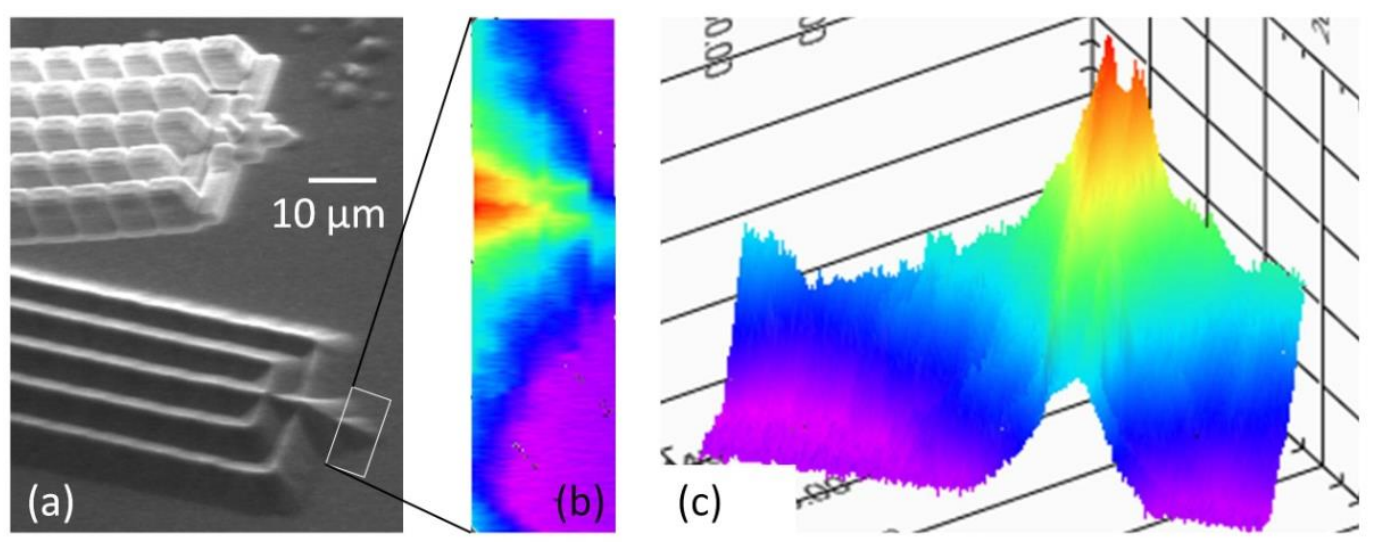

Figure 96: Thermocouple-based SThM Imaging

The second harmonic amplitude set point for the controller must be sufficiently high to overcome the first turning point in the approach curve. This regime of operation corresponds to large temperature excursions from contact. It is therefore unclear how much of the measured signal corresponds to topology, and how much corresponds to heat transfer. A sample with flat topology and significant variation in thermal properties may elucidate this. 


\subsection{Patterning Results}

Using the bolometer-based SThM instrument, a series of indentations was made on wax film deposited on a glass microscope slide. The low melting temperature of the wax combined with the low thermal diffusivity of the glass substrate enables reflow of the media at relatively low temperatures.

In order to generate the patterns, a $300 \mathrm{~Hz}$ AC temperature was generated at the heater and balance resistors, which were placed in a Wheatstone bridge configuration. Over the course of a single line scan, the device was programmed to dwell over 10 equally spaced points. At each point, a tip-sample approach was performed while monitoring the second harmonic amplitude. Contact was assumed to occur after a threshold value was reached, at which point the DC bias on the heater was increased. Upon melting, a large phase change was observed. The controller was therefore programmed to retract after the phase change had been detected. Figure 97 shows several columns of patterned dots, each representing a 5 micron line scan. The patterned features may range in size from 200-500nm depending on the value of the phase set point. Beyond a certain phase threshold, the melted region expands and allows the tip skates forward. The wedge-shaped geometry of the tip is clearly visible in some of the columns in the figure. If the phase threshold is increased further, the melting accelerates rapidly and engulfs neighboring features. If the phase threshold is scaled over the course of a single line scan, the size of the bits increases.

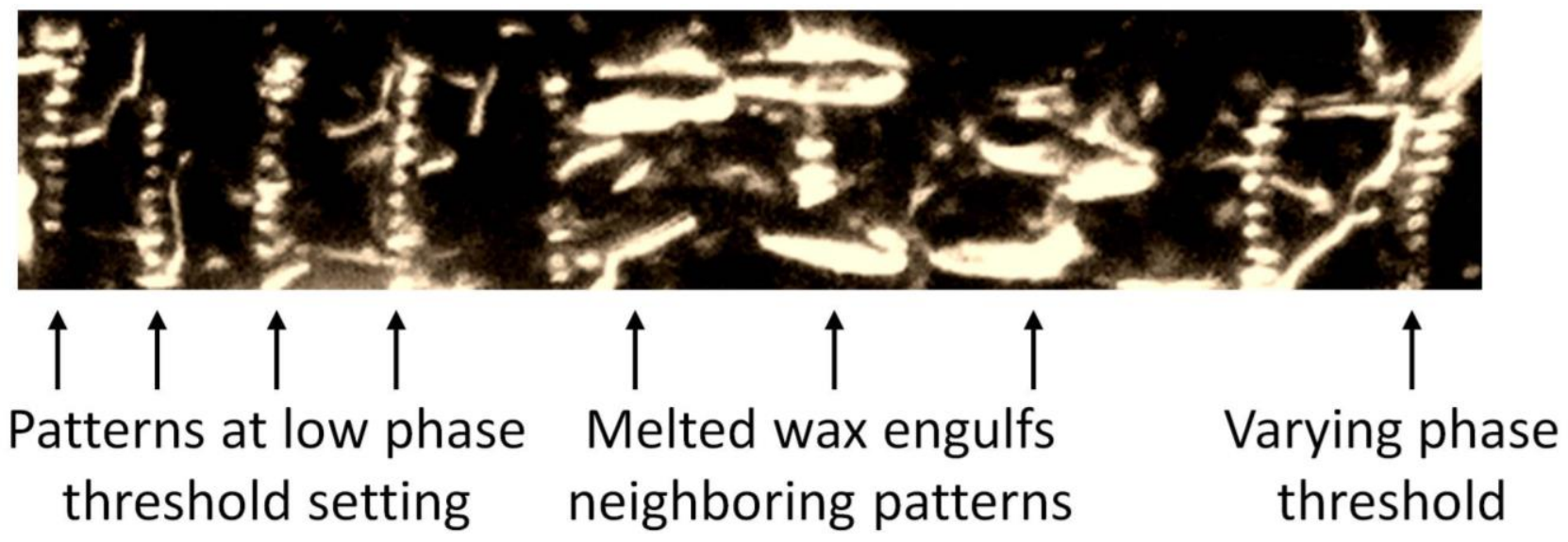

Figure 97: SThM Patterning With a Resistive Heater

The image in Figure 97 was obtained under an optical microscope, since the materials are insulating and cannot be imaged in the SEM's that are available. It would be ideal if the SThM could pattern and image the sample under test. 


\subsection{Summary}

This chapter has presented the first imaging and patterning results with a single-chip SThM. Two measurement techniques have been implemented to measure and modify local thermal properties in the tip-sample region: thermocouple techniques based on the relative temperature between the hot and cold junctions of materials with different Seebeck coefficients, and bolometer techniques based on the TCR of resistive heaters/temperature sensors. In both implementations, differential measurements are enabled through the use of a balanced pair of sensors to improve the rejection of thermal crosstalk from electrothermal actuation. In addition, a resistive heater is located at the base of the sensor beams. If operated at a sufficiently high frequency, this heater modulates the tip-sample temperature difference and the tip-sample gap, enabling a narrow-band measurement at a frequency that is removed from thermal coupling disturbances.

The nature of the tip-sample approach curve that was obtained with a thermocouple probe has been explained with an electro-thermo-mechanical model. Imaging of the topology of a sample was performed with a thermocouple based device. Because the sample consisted of only one material, thermal properties of the sample were not measured.

Resistive temperature sensing in an SThM shares the same noise issues and mitigation strategies as the position sensing methods discussed in Chapter 5. Due to the distributed nature of the thermal transmission line there is a phase difference between the heated base and the tip. This phase difference is modulated by the impedance of the tip-sample gap, and a differential measurement may be used to extract information about local material properties of the sample. Using the phase information as a control signal, a pattern was transferred into a low-melting-temperature wax. 


\section{Chapter 9. Isothermal Scanners and Applications}

By far, the most significant and deleterious effect that has been observed to compromise the performance of single-chip SPMs is thermal crosstalk arising from the temperature excursions of electrothermal actuators. Over the course of this work, several efforts have been made to mitigate this issue, to varying degrees of success:

1. In all of the microscopes discussed herein, balanced cantilever designs have been employed to generate a common-mode signal representing the thermal disturbances and a differential signal that is intended to capture the tip-sample interaction exclusively. Unfortunately, asymmetries in the path of heat flow still exist due to the geometry of the system. When no sample is present, the heat flow from the sensors to the MEMS substrate is asymmetric. When a sample is present, the angle between the cantilever and the sample introduces a gradient in the temperature profile that is unrelated to the quantity being measured. These effects may be calibrated out of the system for a constant temperature, but the temperature varies with the lateral actuator position. These effects compromise the resolution of contact-mode AFMs.

2. A technique based on forced oscillation and higher harmonic detection was introduced to mitigate the thermal coupling issue observed in contact-AFMs. The method involves the measurement of a signal at a higher harmonic of the cantilever oscillation frequency that is generated by the nonlinearity of tip-sample interaction. Unfortunately, in order to achieve sufficient higher harmonic power for closed-loop imaging operation, the tip-sample contact force must be high, and this contributes to significant tip-wear.

1. In dynamic AFM, oscillation of the tip at its natural frequency was used to significantly improve the tip-sample interaction signal. The sensitivity of the natural frequency to thermal coupling is much lower than the sensitivity of the DC output of piezoresistors that experience oscillating temperatures. In addition, the resonant frequencies of the cantilevers are chosen to be beyond the thermal cut-off frequencies of vertical actuators. Under these conditions, a second order effect that is strictly thermomechanical ends up limiting the performance of the instrument: the cantilever height is modulated by the average temperature of the lateral actuators, through heat transfer to the vertical actuator that is used to track topology. This introduces distortions in the image and limits the achievable scan area. 
2. In scanning thermal microscopy, the simultaneous modulation of the tip-sample temperature and gap is used to introduce a second harmonic component to the signal. Unfortunately, the second harmonic amplitude must be set high to overcome the first turning point in the tipsample approach curve. The image thus contains a combination of topography information and thermal information, and the individual components may only be separable with the use of additional information from the phase of the signal. In addition, the image is slanted due to the same second order effect that is observed in dynamic AFM

3. Thermal isolation paths have been used to manage local temperature throughout the devices. The efficacy of these design patterns involves a trade-off between the sensitivity to thermal crosstalk and the SNR that can be attained in position sensors. This is because the efficiency of an actuator improves with thermal isolation, but efficiency is inversely proportional to the SNR of resistive position sensing. The addition of thermal shunt paths may lower the efficiency (and improve SNR) while maintaining good intra-component isolation; however, these paths present a mechanical load to the device and reduce the achievable scan range.

The capability to scan the tip over the sample without changing the local temperature of the sensor would therefore be most welcome. Intuitively, the linear relationship between temperature and position in electrothermal actuators would seem to preclude this scenario. In this chapter, an isothermal electrothermal scanner design is presented that achieves this goal.

\subsection{Device Geometry}

In thermodynamics, an isothermal process is a change of a system in which the temperature remains constant. This term is used loosely here to describe the process in which the change in the system occurs in the mechanical domain, while the temperature at a predetermined location remains constant. An SEM image and FEA simulation of the first isothermal scanner design are shown in Figure 98. 

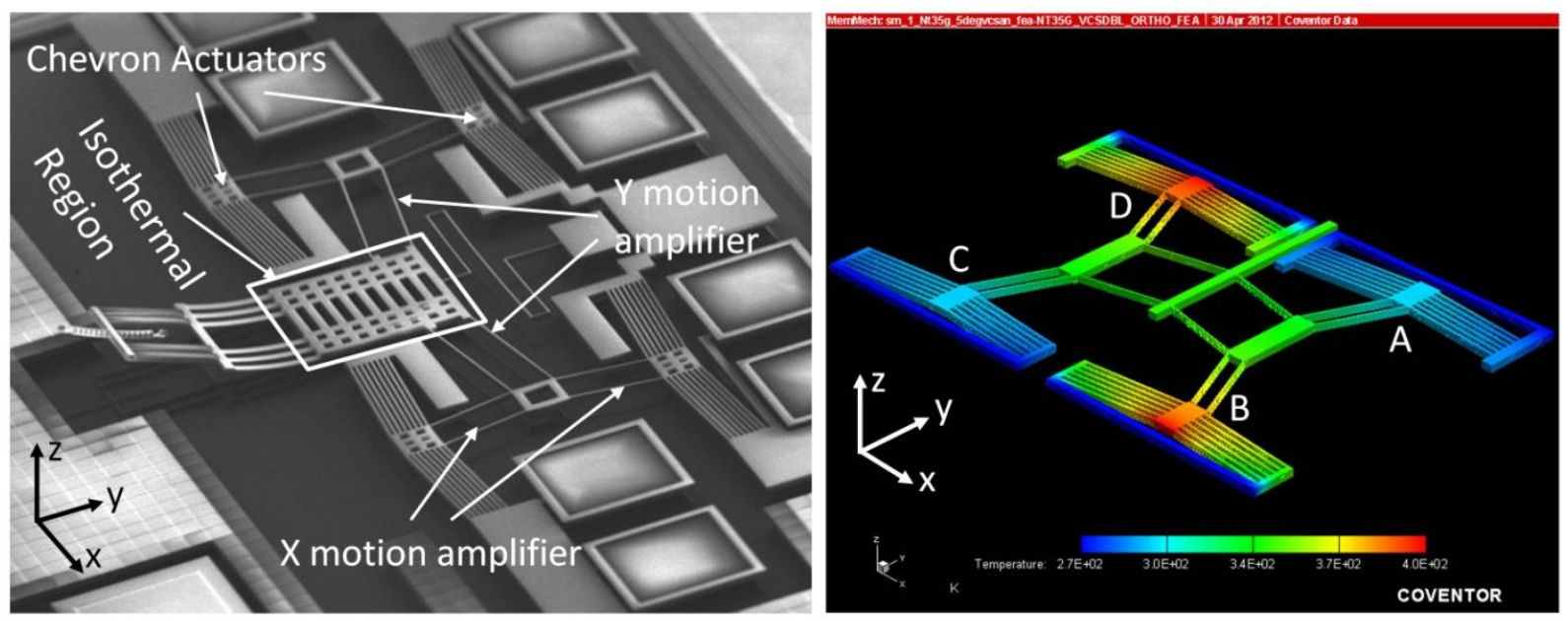

Figure 98: Isothermal Scanner Design

\subsubsection{Chevron Actuators}

The scanner design involves 4 chevron actuators and a flexural suspension that couples them together. The flexural suspension is also designed to amplify the motion of the actuators. The motivation to replace lateral bimorph actuators with chevron actuators in this design is based on a subtle difference between them that was alluded to in section 3.3.3. In bimorphs, the tip-referred force that may be applied is scaled by 4:3 due to the difference between the strain energy stored under a bending moment and the strain energy stored upon tip-loading. However, the design pattern used in lateral bimorph actuators makes use of several symmetrically opposed beams that are grouped together. The tip-referred stiffness of such an arrangement corresponds to the guided-end configuration for each beam, which is $4 x$ stiffer than the cantilevers that are performing the work in the actuator. The situation is exacerbated by the fact that only have of the actuator's beam length is doing work in the desired direction, while the other half is deflecting in the opposite direction. Thus, if one of these actuators is loaded with another, of the same geometry, the resulting range of motion is severely compromised. For chevron actuators, the linear thermal expansion of the beam generates the bending moment. If a chevron actuator is loaded by another one with the same geometry, the deflection is only reduced by $\sim 50 \%$ because the strain energy stored in the deformations of both devices is almost the same. 
The signals that drive the actuators must be properly chosen to achieve isothermal scanning. The constraint that is applied to the drive signals is that the overall power dissipated in the device must remain constant throughout the scan. This constraint ensures that the temperature at the central shuttle remains constant. The signals should also be chosen to produce orthogonal scanning. The additional constraint that ensures rectilinear scanning is that pairs of actuators ( $A B$ and $C D)$ should experience differential inputs. A set of FEA results that demonstrates this concept is tabulated below (Table 4).

Table 4: FEA Simulation Results of Isothermal Scanner

\begin{tabular}{|c|c|c|c|c|c|c|}
\hline $\begin{array}{c}\text { Actuator A } \\
\text { Temperature (K) }\end{array}$ & $\begin{array}{c}\text { Actuator B } \\
\text { Temperature (K) }\end{array}$ & $\begin{array}{c}\text { Actuator C } \\
\text { Temperature (K) }\end{array}$ & $\begin{array}{c}\text { Actuator D } \\
\text { Temperature }(\mathrm{K})\end{array}$ & $\begin{array}{c}\text { X position } \\
(\mu \mathrm{m})\end{array}$ & $\begin{array}{c}\text { Y position } \\
(\mu \mathrm{m})\end{array}$ & $\begin{array}{c}\text { Temperature of } \\
\text { Tip (K) }\end{array}$ \\
\hline 400 & 400 & 400 & 400 & 0.001 & 5.120 & 400.00 \\
\hline 500 & 300 & 300 & 500 & 0.000 & -3.447 & 400.01 \\
\hline 350 & 450 & 450 & 350 & -0.002 & 10.528 & 399.90 \\
\hline 500 & 300 & 500 & 300 & 3.742 & 3.002 & 400.03 \\
\hline 300 & 500 & 300 & 500 & -3.740 & 2.997 & 399.97 \\
\hline 350 & 450 & 350 & 450 & -1.876 & 4.544 & 399.99 \\
\hline 450 & 350 & 450 & 350 & 1.877 & 4.544 & 400.15 \\
\hline
\end{tabular}

The FEA results illustrate the preferred mode of operation of the device. First, the average temperature of the scanner is chosen. In this simulation, the average value was set to $400 \mathrm{~K}$, resulting in an offset position that will be discussed later. To position the payload in the positive $\mathrm{Y}$ direction, one may "pinch" the pairs of actuators closer together. In other words, for the AB pair of actuators, the power in actuator A should be reduced while the power in actuator B should be increased. Similarly, for the CD pair of actuators, the power in actuator $D$ should be reduced while the power in actuator $C$ should be increased. This brings the both pairs of actuators closer to one another, and the flexures amplify the "pinching" motion to a $Y$ deflection. The converse situation corresponds to scanning in the negative $Y$ direction. The first three entries in the table correspond to $Y$ scanning. Notice that the $X$ position is predicted to remain constant to within a few nanometers, and the temperature at the tip remains constant to within $0.1 \mathrm{~K}$. 
To position the payload in the positive $\mathrm{X}$ direction, one pair of actuators should be "pinched" while the other pair should be pulled apart. In other words, for the AB pair of actuators, the power in actuator $A$ should be increased while the power in actuator B should be reduced. Similarly, for the CD pair of actuators, the power in actuator $D$ should be reduced while the power in actuator $C$ should be increased. This "pinches" the CD pair while pulling apart the AB pair, and the flexures amplify the motion in the $X$ direction. The last 4 entries in the table correspond to $X$ scanning. Notice in this case that the temperature at the tip remains constant to within $0.1 \mathrm{~K}$. However, the $\mathrm{Y}$ position does not remain constant, which is one of the parasitic effects that will be discussed next.

\subsection{Isothermal Scanner Parasitic Effects and Mitigation}

The measured performance of the scanner under $1 \mathrm{~V}$ of actuation is reported here. A single-ended measurement at the tip thermocouple was obtained with a sample present to provide a cooling path. The device offers excellent isothermal performance over its scanning range. When scanning in an isothermal fashion, the resulting range of thermocouple outputs is $\sim 40 \mathrm{~dB}$ below the range of values that occurs when all 4 actuators are simultaneously actuated with the same maximum input power. A scan range of $\sim 10 \mu \mathrm{m} x \sim 5 \mu \mathrm{m}$ was observed under an optical microscope, with no observable deflection of the Z-actuator. Z-actuator deflection is easy to identify in these structures as a shift in the reflected pattern of the illumination source of the optical microscope due to small changes in the curvature of the beam. Although the isothermal measurements reported here are anticipated to significantly improve singlechip SPM performance, several parasitic effects were observed.

\subsection{Observed Parasitic Effects and Mitigation Strategies}

From FEA and measurements, it was observed that although the scan pattern is intended to be orthogonal, parasitic motion in the $\mathrm{Y}$ direction occurs and distorts the $\mathrm{X}$-direction of the scan into an arc. The $\mathrm{Y}$-direction scan remains linear. This can be explained by the fact that the flexures experience a temperature gradient from the differential power in the actuators, resulting in self-actuation of the flexures. The gradients in the X-motion amplifiers are symmetric or antisymmetric in this mode of operation, so there is no distortion in the $X$ direction. However, the difference in $X$-amplifier selfactuation is amplified by the $\mathrm{Y}$-motion amplifier. The $\mathrm{Y}$-amplifiers own self-actuation also contributes to the distortion. 
A simple solution exists to mitigate this issue. First, the scan may be performed in the Y-direction, so that individual line scans are unperturbed. Each line may then be shifted and scaled, performing a coordinate transformation that removes the distortion in the image.

Another parasitic effect is related to the mismatch in the resistance of the actuators due to well-known variations in sheet resistance that are quantified in the CMOS design kit. If the same square-root voltage is applied to different-valued resistors, the resulting power will not be matched.

There are two simple solutions to this problem. First, the resistance is measured. Then, the applied voltages may be scaled to improve power matching. Alternatively, off-chip trimming resistors may be placed in parallel with the devices for voltage drive, or in series for current drive. To first order, these solutions also account for TCR effects that occur during operation of the scanner.

The most significant parasitic effect results from vertical residual and thermally induced stresses in the chevron actuators. In order for the bending moments about the z-axis in a chevron actuator to perform mechanical work, a high aspect ratio is desirable to prevent buckling in the out-of-plane direction. In addition, the angle of the chevron beams must be chosen to prevent buckling due to compressive loads in the in-plane direction. During operation, it was observed that if powers above $10 \mathrm{~mW}$ are applied, the scanner deflection no longer increases. Above $160 \mathrm{~mW}$, the combination of stress and high temperatures cause permanent plastic deformation in the metal layers and results in a new out-of-plane "self-assembly" curvature that permanently degrades device performance. To the best of the author's knowledge, these thermal and residual stress-related effects have precluded the use of chevron actuators in CMOS-MEMS processes.

\subsection{Amplified Strapped Chevron Scanner}

To counter the out-of-plane bending from residual and thermal stresses, the chevron actuator may be anchored through a set of flexures as shown in Figure 99. The four flexures, arranged similarly to a crab spring, are compliant in the lateral direction but stiff in the vertical direction, improving the overall stiffness of the actuator. In addition to preventing out-of-plane buckling, they provide an additional thermal shunt path and an electrical routing path that does not experience high temperatures.

Chevron actuators do not produce the same deflections as bimorphs. A mechanical amplification structure was sought to improve the scan range of the actuator while maintaining high stiffness and a 
small footprint. The geometry of the amplifying flexures in Figure 99 meets these requirements.

\section{Isothermal Scanners}
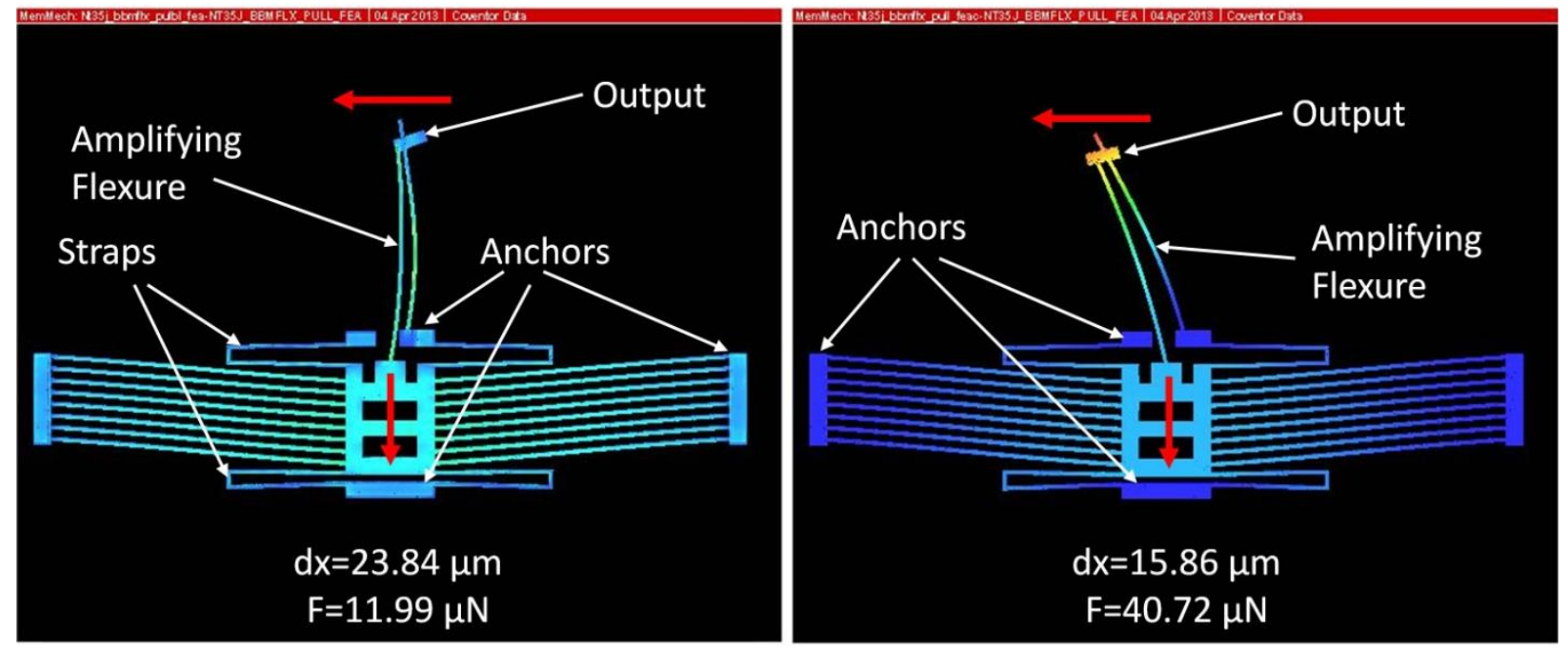

Figure 99: Amplified Strapped Chevron Actuator. FEA of Displacement and Blocked Force

The angle of the amplifying flexure significantly impacts the overall performance of the actuator. A figure of merit for actuators is the work-per-unit-volume, which is the product of the blocked force and output displacement. The initial angle of the flexure on the left side of Figure 99 makes it more susceptible to buckling while it is under a compressive load from the downward movement of the chevron. As a result, work-per-unit-volume of the actuator on the right is $225 \%$ greater. Both actuators provide a direct thermal shunt to their payload through anchor of the flexure. Thermal isolation may be added in between the chevron and the flexure to improve the situation further.

To put these FEA results into perspective, the same analysis is performed for a lateral multimorph actuator with the same footprint. The work-per-unit-volume of the device in Figure 99 is $>2500 \%$ higher than that of the lateral multimorphs used in single-chip SPMs thus far. The out-of-plane stiffness of the new actuators is also improved by a factor of roughly $350 \%$.

\subsection{Amplified Strapped Chevron Scanner}

A single-chip multi-mode SPM was designed with a scanner based on the lateral actuators discussed above. The device makes use of piezoresistive strain sensors that are thermally balanced, and produce a differential strain signal. The differential strain signal is produced by converting the moment from tiploading into a torque, and then placing the piezoresistors on opposite sides of the center of rotation. Thus, one resistor experiences compressive strain while the other is in tensile strain. The thermal 
matching of the piezoresistors is also improved, since they are equidistant from the substrate. The design and optimization of these of these strain sensors is beyond the scope of this thesis. The device also contains a balanced pair of temperature sensors at its tip. The goal of the device is to perform SThM while using a dynamic AFM to maintain a constant tip-sample distance. This section focuses on the scanner design in Figure 100.

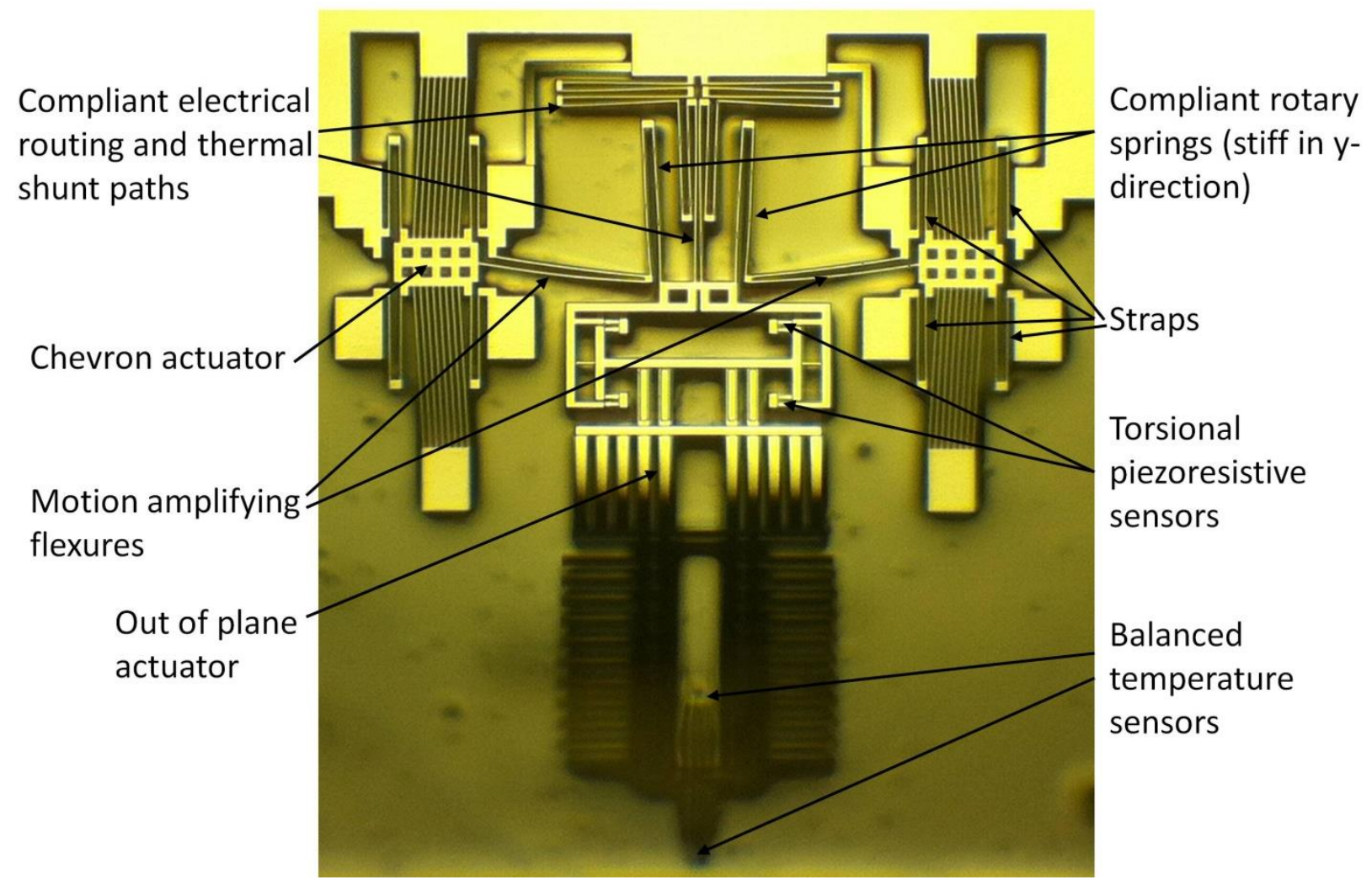

Figure 100: Scanner Based on Amplified Strapped Chevron Actuators

With $10 \mathrm{~mW}$ of input power, the new scanner design provides a lateral displacement of $\sim 40 \mu \mathrm{m} X 10 \mu \mathrm{m}$ and has a vertical scan range is $>20 \mu \mathrm{m}$. The residual tensile stress in the metal layers results in a selfassembly displacement at the output of the actuators of $\sim 10 \mu \mathrm{m}$, which can be observed in the bent shape of the flexures and thermal shunt paths. A single-axis lateral scan was performed in the isothermal mode discussed above, by applying a differential signal to the actuators while maintaining a constant total power. In the orthogonal lateral axis, isothermal scanning cannot be performed with this device; however, very little coupling was observable in the deflection of the vertical actuator upon nonisothermal actuation. This is in part because of the large number of thermal shunt paths that are provided for the vertical actuator, and also due to convective cooling provided by fins that are 
integrated in the cantilever. The integrated temperature sensors may be used to quantify the amount of thermal coupling. The scanner bandwidth of is also improved by short paths to thermal ground and the reduced thermal capacitance of the actuators.

\subsection{Applications of Isothermal Scanners}

All of the mitigation strategies for thermal crosstalk that have been developed in this work stand to benefit from isothermal scanning. In contact AFM, isothermal scanners stand to improve vertical resolution and strengthen the efficacy of the balanced cantilever approach, by offering a constant operating temperature and thus permitting a one-time calibration to null the offset voltage from the balanced cantilevers. In the higher harmonic detection scenario, these scanners may reduce the minimum detectable power of higher harmonics. In dynamic AFM, the effects of the temperature coefficient of resonant frequency will be suppressed, thereby improving the resolution of the devices. In scanning thermal microscopy, the minimum detectable thermal interaction may be improved by reducing scanner-induced temperature variations. The improved work-per-unit-volume of the chevrons enables the addition of more thermal shunt paths, thereby enabling design patterns with vertical actuators that are thermally isolated from sensors and lateral actuators.

The isothermal scanners may also improve the performance of devices that have not been discussed in this thesis. For example, single-chip scanning microwave microscopes were designed, fabricated and characterized over the course of this work [184]. Measurements obtained with these devices confirm that the series resistance of the microwave signal path greatly impacts the reflected signal power S11, which is measured to quantify local dielectric and conductive material properties of the sample. The devices that have been presented thus far have made use of narrow transmission lines with high series resistance, to reduce the mechanical load on the actuators. The use of the scanners presented in this chapter will enable a significant reduction of the series resistance in the microwave signal path. In addition, the resistance is modulated by the temperature of the scanners; this modulation will be greatly suppressed through the use of isothermal scanners.

Single-chip scanning tunneling microscopes have also been fabricated over the course of this work. The goal of the design was to implement arrays of STM's for atomically precise manufacturing (APM). 1D array implementations like the one shown below were used to obtain measurements under vacuum conditions. The thermal coupling between adjacent devices was observable in an SEM. At the length scales of APM, the magnitude of thermal coupling is considered to be severe. In the depiction of a 2D 
array in Figure 101, each STM is intended to operate independently with its own integrated scanner. This type of operation would give rise to substrate temperature variations that would be difficult to predict and compensate. The isothermal scanner design ensures that the power dissipated by each scanner remains constant, thereby suppressing thermal crosstalk between a large number of devices integrated on the same substrate.
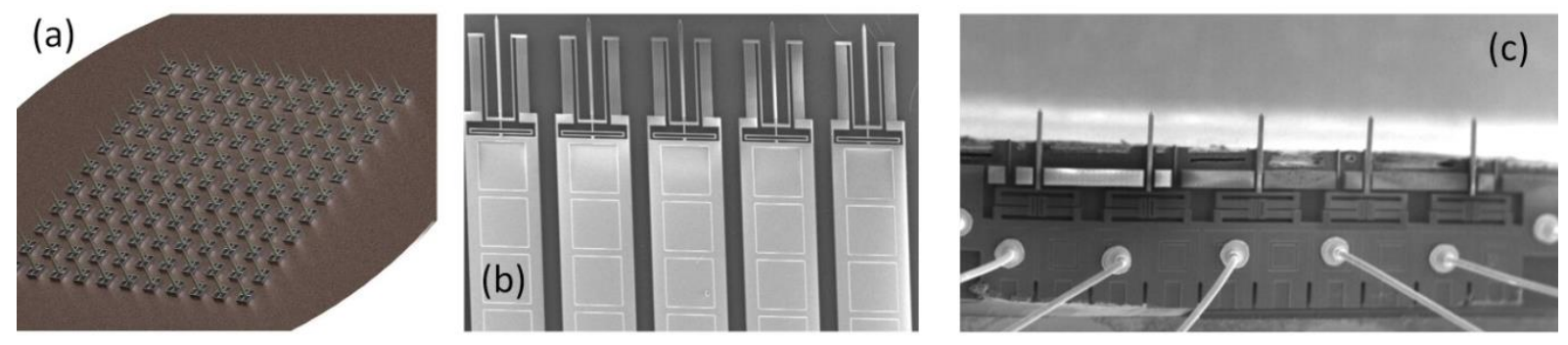

Figure 101: Depiction of a 2D Array of STM's (a), 1X5 Array of CMOS-MEMS STM's (b), Similar STM Array Suspended Over Chip Edge (c) 


\section{Chapter 10. Conclusion}

Scanning probe microscopes are widely regarded as the workhorse instruments of nanoscience because of their unsurpassed imaging resolution and their capability to pattern with exquisite dimensional control; however, there are several limitations in the design and construction of conventional SPMs that have averted their widespread adoption by the metrology community. The original motivation for this work was to aggressively miniaturize and integrate all of the elements of a conventional SPM instrument onto a single chip. Scaling laws indicate that smaller dimensions would enable SPMs with higher bandwidth, improved stability, and better overall performance. Simultaneously, the use of batch fabrication in conventional CMOS technology could significantly lower the cost, permit array operation, and offer unprecedented levels of integration, which could improve the ease-of-use of single-chip instruments.

Incremental progress began with the first AFM images captured in contact mode [185],[186]. These results were sufficient to validate the concept; however, they revealed several severe shortcomings of the single-chip SPM approach. Electrothermal actuation provided sufficient static and dynamic displacements, but the signal from parasitic thermal crosstalk overwhelmed the signal from tip-sample interaction.

When the tip was forced to oscillate at a frequency near its thermal pole, it was observed that the signals from abrupt tip-sample contact included higher harmonic frequency content. A method was therefore sought to capture an image based on these observations, in the hopes that the lower frequency thermal coupling effects could be suppressed [187]. Although the method produced superior images, it revealed deeper issues with the single-chip SPM approach. Due to the low gauge factors of CMOS polysilicon, large tapping forces were required in order to obtain sufficient signal-to-noise ratio for imaging operation. The high tip-sample interaction forces resulted in significant tip wear.

Dynamic modes of operation were then developed in order to increase the tip-sample force sensitivity and thereby reduce tip wear [188]. AM-AFM and FM-AFM were both observed to improve imaging performance significantly. $90 \mathrm{~nm}$ vertical resolution was obtained with AM-AFM at scan rates of $1 \mathrm{~Hz}$. Imaging with FM-AFM was also performed with further improved vertical resolution, and revealed that a more sophisticated controller or a stiffer instrument would be required to prevent pull-in effects in this mode. Despite the improved resolution, it was noted that the material properties and geometry of laminated mechanical structures in CMOS-MEMS processes imposed an upper limit on the Q-factors 
that could achieved under ambient conditions. In addition second-order effects, such as the thermomechanical coupling of heat from lateral actuators into the position of vertical actuators, emerged as the dominant disturbances that were observed to distort images and limit the achievable scan range.

The use of thermocouples and resistive temperature sensors was explored next, since signals from thermal origins seemed to dominate the tip-sample interaction measurements. Two types of SThMs were thus designed, fabricated and characterized. Both patterning and imaging using SThM were performed with these devices. At this point it was believed that the single-chip SPMs could find applications in niche areas, where their simplicity, size and low-cost may be advantageous.

Over the course of this work, the development of simplified lumped element models has aided in the interpretation of experimental results and has guided the design of incrementally improved devices. Based on the knowledge of CMOS-MEMS material properties and some insight into the electrical, thermal and mechanical effects in the instruments, a pragmatic position was taken when comparing single-chip SPMs to the state-of-the-art. It was believed that these instruments may not achieve the performance of state-of-the-art tools, but that they could be applied where resolution could be compromised in exchange for higher speed, smaller form factor, simplicity and lower cost.

To improve the performance of thermal detection techniques, isothermal scanner designs were developed. Devices incorporating these design patterns were found to successfully mitigate a large component of the thermal crosstalk. In addition to reducing temperature variations, the designs provide a more robust mechanical scanning platform in terms of stiffness, position sensitivity, and output force. It is believed that all of the modes developed to date may benefit from such a scanner design.

A method to markedly increase the quality factor ( $Q$-factor) of flexural resonators was then discovered. The approach relies on an internal energy pumping mechanism that is based on the interplay between electrical, mechanical, and thermal effects. Flexural resonances in air with unprecedented Q-factors were observed, and a physical explanation for the underlying mechanism was proposed.

With the advent of high-Q resonators and robust isothermal scanning, it is now believed that single-chip SPMs may achieve resolution that is commensurate with conventional instruments. A moderate Qfactor was required to achieve the resolution reported for AM-AFM in air, and it is likely that performance will improve significantly upon application of recently developed design patterns. 
Present and future work aims to systematically leverage the results in this dissertation. To exploit the versatility of isothermal scanners, a modular layout cell now exists to accommodate AFM, SThM, KPFM, SMM, MFM, and STM prototypes. To capitalize on Q-pumping, a series of test structures will be designed to foster a deeper understanding of the phenomenon, quantify the dominant effects, and initiate an optimization effort. To improve the resolution of the instruments, process development effort will aim to produce sharp tips with improved wear-resistance in a parallel fashion. Fielddependent sputter sharpening and CVD of Hafnium Diboride are likely candidates for tip development [189]. For array operation and enhanced ease-of-use, the instruments should be implemented in a CMOS process that offers through-silicon-vias; such a process would obviate the need for a coarse approach mechanism, permitting the MEMS to be placed directly on a sample. Single-chip SPMs with consistently high $Q$, reliable isothermal scanning operation, and sharper tips represent an ambitious yet achievable goal.

This research has demonstrated that it is indeed possible to integrate entire scanning probe microscopes onto a single chip. Experimental results suggest that the performance of the single-chip instruments has benefitted from dimensional scaling. Recent developments have offered mechanisms to overcome many of the limitations that were identified over the course of this study. It is believed that this is just the beginning of a path towards tiny, inexpensive, fast, and high resolution SPMs that will augment the state-of-the-art in metrology today.
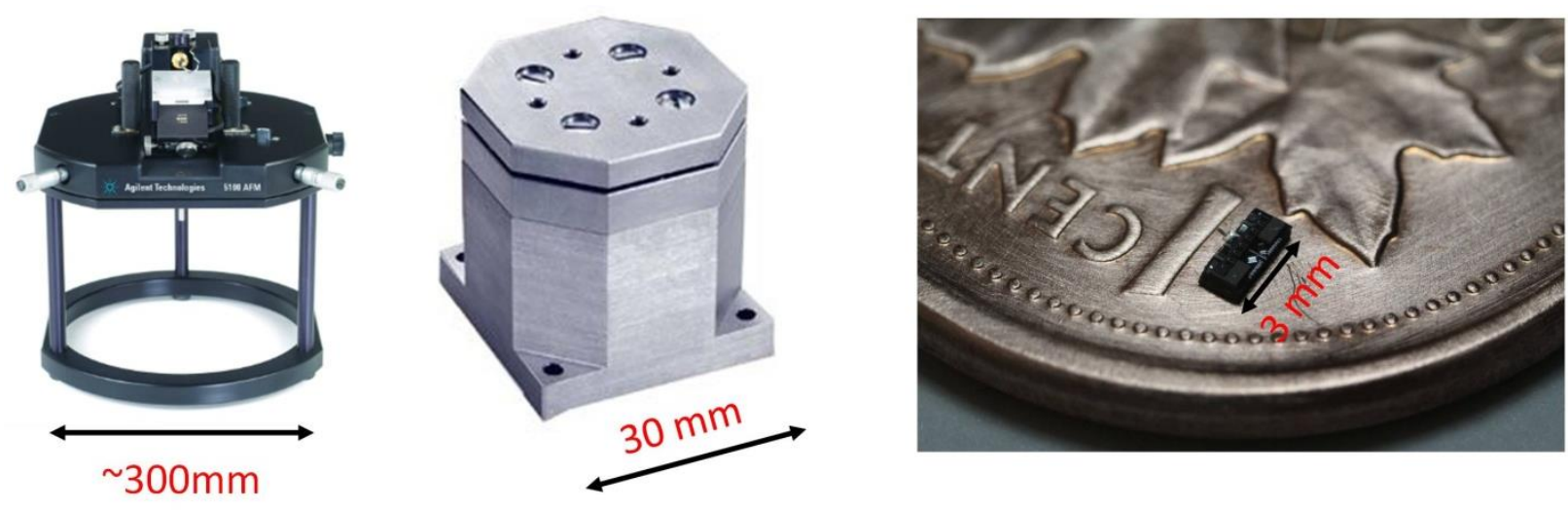


\section{Appendix A MEMS Design Philosophy and Scaling Laws}

The hallmark of a well-designed microsystem is a fundamental understanding of the underlying physics that is confirmed by good agreement between predictive models and measured results [190]. The electrical, thermal, and mechanical behaviour of the components of the instrument must first be revealed by models that predict device performance. The design cycle continues with a comparison of these predictive models to the unforgiving results of characterization. Material properties are established, new effects are discovered, and parameters are identified. These quantities are used to refine and tune the model to improve its predictive power for ensuing design cycles. Simultaneously, the model is used to guide the optimization of the next design cycle. A challenging yet rewarding aspect of CMOS-MEMS SPM design is that there are no parametric libraries in the suite of CAD tools available to the designer. Thus, the success or failure of a device can be attributed directly to the quality and rigor of the design process.

As in all engineering disciplines, a balance must be struck between the effort invested in accurate quantitative predictive models, and the penalties associated with fabrication. Examples of penalties are the length of the fabrication cycle (2-3 months of time) and the cost of space on a CMOS shuttle run $\left(\sim \$ 00 / \mathrm{mm}^{2}\right)$. This balancing act dictates the number of engineering revisions, or design cycles, that is acceptable to achieve a desired set of specifications. The following sections discuss the nature and distribution of effort in the design of chip-scaled instruments from simulation, to fabrication, to characterization.

\section{A.1 Simulation}

There is a spectrum of simulation strategies that ranges from approximate, intuitive and fast Lumped Element Models (LEM) to accurate, computationally expensive Finite Element Models (FEM).

In this work, the constitutive equations are first examined in order to identify dominant effects. Simplifications are made through discretization to develop electro-thermo-mechanical lumped element models (ETM-LEM's). The design intuition imparted by LEM's is of great value. At a glance, a schematic representation of the LEM offers insight into various tradeoffs. These models can be solved using a variety of freely available Spice-like solvers. Optimization tools that are built into these solvers are most useful in guiding the MEMS design process. These tools are also used to identify material properties in the fabrication process. 
Accurate predictions of quantities like resonant frequencies and 3-dimensional temperature distributions are obtained with an FEA solver. Numerical techniques are well-suited to the solution of non-linear quantities that contribute to the static and dynamic behaviour of electrothermally excited cantilevers. FEM techniques should also be used when the number of elements is so large that it would obscure the insight that one could gain from a lumped model, as is the case with temperature distribution in 3D. Careful application of boundary conditions and symmetry considerations are effective ways to reduce the simulation time associated with the FEM approach. The typical length of FEM simulations in this work is 1-10 minutes.

In the case of heat transfer through beams, transmission line models that are simplifications of large LEM's are used as a compromise between LEM and FEM approaches.

In the case of electromagnetic simulations, commercially available finite difference time domain (FDTD) solvers have been used in conjunction with lumped element models that are valid in the microwave regime.

\section{A.2 Fabrication}

The performance of a MEMS device is inexorably tied to its fabrication process. Material properties and geometrical constraints have a direct impact on the specifications that can be met. For this reason, there is no standard process that can simultaneously address the needs of the plethora of MEMS devices that are commercially available or under investigation today. Most commercial MEMS products therefore have a unique, proprietary fabrication process.

Multi-user MEMS processes are designed to be versatile so that various designs can be prototyped; however, there is a trade-off between versatility and device performance. The material properties of such processes are seldom optimized for a particular application. Chip-scaled SPM instruments rely on the concerted operation of various sub-components, each of which exploits a range of material properties. To position the tip in the plane of the substrate, lateral electrothermal actuators are used. Vertical displacement is achieved with out-of-plane electrothermal bimorph actuators. These actuators require two materials with different thermal expansion coefficients. Flexure mechanisms are used to mechanically couple the actuators to each other and to the tip. These mechanisms also comprise electrical routing and serve as thermal conductors or isolators. The flexures therefore rely on materials with electrical and thermal insulating and conductive properties, as well as favorable mechanical properties. The tip-sample interaction forces are measured using various different types of sensors. In 
AFM, the tip-sample interaction forces are detected by piezoresistive strain gauges. A resonant cantilever improves the sensitivity of the force measurements, by providing some gain in the mechanical domain. Thus, the cantilevers require piezoresistive materials and mechanical properties that influence resonance frequency and quality factor. In SThM, resistive thermometers or thermocouples based on the Seebeck effect can be used to measure thermal effects. These measurements rely on the polysilicon material's temperature coefficient of resistivity (TCR) and Seebeck coeffients. In STM, the tunneling current between a sharp, conductive tip and the sample is measured with a low-noise amplifier. For this mode to work in ambient conditions, the tip material should not be susceptible to the formation of a native oxide layer. In SMM, a high frequency signal path to the tip is needed to measure small changes in the tip-sample impedance. The microwave material properties of the conductors and insulators in the signal path are thus of great consequence. Every aspect of the design of a CMOS-MEMS SPM leverages material properties that are offered by the process.

\section{A.3 Characterization}

It is difficult to measure the tiny quantities that result from tip-sample interactions in the nanometer range. Precautions must be taken both in the design of the devices and in the design of the measurement setup to suppress the effects of noise in the electrical, mechanical, and thermal domains.

In the electrical domain, several problematic noise sources obscure the measurement of currents and voltages. Senturia [149] discusses the sources of electrical noise and techniques to suppress them. Electrical shielding reduces the amount of electromagnetic interference that may couple to signal lines. Shielding must also be used to prevent coupling of drive and sense lines, both within the device and in external wiring. Ground loops are eliminated by tying all signal grounds to a single point. The resistance of the routing to and from sensors and actuators within a device is problematic and should be kept low whenever possible. For differential signals, this resistance should be matched. In some cases the spectral density of electrical noise shows frequency dependence. For example, $1 / f$ noise decreases with frequency, and noise from wall outlets occurs at $60 \mathrm{~Hz}$. Lock-in techniques can be used to perform measurements at a frequency where the noise spectral density is low, and to limit the bandwidth of the measurement. Batteries are a good choice of power supply when compared to regulated power supplies, due to the improved noise performance and low output impedance that they offer.

In the mechanical domain, acoustic and building vibrations contribute noise to the physical tip-sample separation. Vibration isolation techniques are important in the design of various scientific instruments, 
as discussed in Park and Quate [191] and de Silva [192]. Scaling of the dimensions in a CMOS-MEMS SPM dramatically improves its immunity to these sources of noise. Nevertheless, several techniques for vibration isolation and acoustic shielding have been used in this work. The highest resolution images obtained with these instruments to date have been acquired without vibration or acoustic isolation. Thermomechanical noise in cantilevers, the mechanical analog of Johnson noise, is another consideration.

In the thermal domain, ambient air drafts and thermal coupling within devices are the dominant sources of noise. Vacuum operation eliminates thermal coupling through air conduction. Substrate heating results in long-term drifts that can be mitigated through temperature control techniques. Finally, design patterns can be used to reduce the effects of temperature variations near sensors.

Various laboratory instruments are used in the characterization process. The static and dynamic motion of scanners is measured in the vacuum environment of a scanning electron microscope (SEM) that was retrofitted with an electrical feedthrough. A stroboscopic MEMS motion analysis tool is used to capture resonance amplitudes and static displacement amplitudes in air. A white light interferometer is used to quantify the out-of-plane curl due to residual stress in the films. A vector network analyzer (VNA) is used to measure small changes in the tip-sample impedance at microwave frequencies. A lock-in amplifier is used to limit the noise bandwidth in the measurements, and a PLL is used to track the resonant frequency of a cantilever in the FM mode. The control system is implemented on an FPGA card with digital and analog inputs and outputs. A MEMS interface board contains drive and sense electronics that are designed specifically for the MEMS devices.

In air, various jigs are used for coarse positioning of the sample under the instrument. A vacuum probe station with RF and DC feed-throughs is used to perform imaging experiments at low pressures. Ultimately, the device performance must be characterized by performing imaging experiments with calibration samples.

\section{A.4 Scaling Laws}

A fundamental assertion of this dissertation is that the performance of SPM's stands to benefit from miniaturization. In fact, this assertion is of importance to all MEMS endeavours; the laws of scaling merit discussion. In this section, examples are presented that are of particular relevance to the design of SPM's: 
1) Scaling of power and thermal time constants associated with microscale electrothermal actuators

2) Scaling of inertial forces and droop in microscale spring-mass suspensions

3) Scaling of resonant frequency and Quality $(Q)$ factor of microscale cantilever resonators

In each case, the constitutive equations are expressed in terms of dimensional quantities and a scaling factor is introduced to assess the impact of miniaturization.

\section{A.1.4 Thermal Time Constants}

The scanners that are used throughout this work are based on the principle of electrothermal actuation. The bandwidth and power consumption of these actuators improves dramatically with scaling. Consider the amount of energy required to elevate the temperature of the cube below by $1^{\circ} \mathrm{K}$.

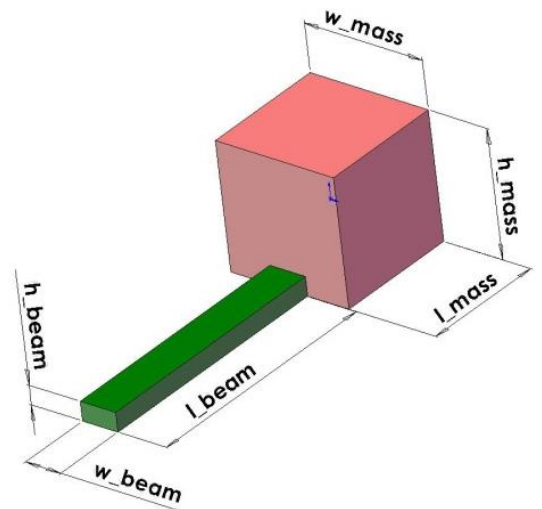

If the cube is thermally isolated, the energy is:

$$
\Delta E=l w h \rho c_{p} \Delta T
$$

where the dimensions are indicated in the figure, $\rho$ is the density and $c_{p}$ is the specific heat capacity of the material. If all dimensions are scaled by a factor $s$, we can rewrite equation 1 as:

$$
\Delta E=\operatorname{sl} s w \operatorname{sh} \rho c_{p} \Delta T=s^{3} l w h \rho c_{p} \propto s^{3}
$$

To put this in perspective, the CMOS-MEMS SPM actuators are 100x smaller in all dimensions than a conventional scanner, so the energy required to heat the MEMS cube is $1,000,000 x$ lower. The 
miniscule amount of energy required to heat up a micro-scale device is what enables the operation of electrothermal actuation, which would not be an option in meso-scale or macro-scale devices.

In reality, the cube cannot be perfectly thermally isolated. Heat loss to the surroundings through convection and to the substrate through conduction must be considered. The efficacy of convective cooling is related to the surface area to volume ratio, which is expressed as:

$$
\frac{\text { Surface area }}{\text { Volume }}=\frac{6 l^{2}}{l^{3}}=\frac{6}{l} \propto \frac{1}{S}
$$

This means that the surface to volume ratio increases by a factor of 100 if the dimensions are scaled down by a factor of 100 . Convective cooling is thus $100 x$ more efficient at the length scale of interest.

Finally, the conductive heat path to the substrate must be considered. The geometry in the figure above includes a cantilever beam that suspends the thermal mass. The thermal resistance of this beam is expressed as:

$$
R=k \frac{l}{w h}
$$

where $k$ is the material property of thermal conductivity. Scaling the dimensions of this beam results in the following expression:

$$
R=k \frac{s l}{s w s h} \propto \frac{1}{s}
$$

The thermal resistance of the beam increases in proportion to downward scaling, similar to the case of convective cooling. An equivalent circuit that captures the energy storage as a capacitance, and the convective and conductive cooling as resistances was shown in Figure 3. A current source was used to represent the heat generated within the thermal mass. This lumped element approximation can be used to describe the thermal dynamics of the mass in familiar terms. In this analogy, the voltage represents temperature and one can calculate the thermal response as the solution to the nodal voltage equation:

$$
\Delta T=\Delta T_{f}\left(1-e^{\frac{-\left(t-t_{0}\right)}{R C}}\right)
$$

The product $R C$ that appears in the denominator of the exponential is known as $\tau$, the thermal time constant. The scaling of this time constant for the geometry under consideration is: 


$$
\tau=R C=k \frac{l}{w h} \times l w h \rho c_{p} \propto \frac{s^{4}}{s^{2}}=s^{2}
$$

This implies that if the dimensions are scaled by a factor of 100 , the time constant is reduced by a factor of 10,000 . The plots in Figure 13 identified the $-3 \mathrm{~dB}$ frequencies associated with beams that have different RC time constants. The designer may parametrically vary the dimensions of the constituent elements to tune the $\mathrm{R}$ and $\mathrm{C}$ values to reduce power consumption or increase bandwidth. This example illustrates that electrothermal actuation at the micron scale can attain bandwidths and power levels that are competitive with conventional mesoscale scanners. At the nanometer scale, they have been shown to excite resonances in excess of $150 \mathrm{MHz}$.

\section{A.2.4 Stiffness and Droop}

Flexures are used throughout this work to amplify displacements and support micro-scale payloads. The geometry that was considered in the preceding section serves as an example of a mass suspended by a flexure. The length of the resistive beam is a variable that can be manipulated to increase thermal resistance in order to improve the thermal isolation of the mass, and reduce the amount of power needed to heat it. At larger scales, this geometry becomes impractical due to inertial forces.

Consider the scaling of the stiffness of a cantilever beam subject to a point load at its tip:

$$
k=\frac{E w h^{3}}{4 l^{3}} \propto \frac{s^{4}}{s^{3}}=s
$$

The stiffness scales linearly with size. The displacement of the cantilever due to an inertial force exerted by the load is calculated from:

$$
F=k x
$$

The inertial force $\mathrm{F}$ is:

$$
F=m(g+a)
$$

where $g$ is the gravitation constant. The scaling of the displacement $x$ follows:

$$
x=\frac{F}{k}=\frac{l w h \rho(g+a)}{s} \propto \frac{s^{3}}{s}=s^{2}
$$


The serpentine springs that are used throughout the design of CMOS-MEMS SPM's are only practical because of this scaling effect. If the size of the scanners is increased by a factor of 100 , the displacement or "droop" increases by a factor of 10,000. A $1 \mu \mathrm{m}$ droop at the MEMS scale would correspond to a $10 \mathrm{~mm}$ droop at the scale of conventional scanners. The robustness of a device when subjected to external accelerations is directly related to this quantity as well.

\section{A.3.4 Resonant Frequency and Quality Factor}

In dynamic AFM, a resonant cantilever is used to measure tip-sample interaction forces. A high resonant frequency contributes to improved bandwidth of the measurement. A simplified expression for the resonant frequency of a cantilever beam is:

$$
\omega_{0}=2 \pi f=\sqrt{\frac{k}{m}}
$$

This expression corresponds to a lumped element model that ignores distributed effects. For the qualitative purpose of evaluating scaling in the resonant frequency, this model produces the same result as a distributed model. When scaled by a factor $s$, the resonant frequency follows:

$$
\omega_{0}=\sqrt{\frac{k}{m}}=\sqrt{\frac{s}{s^{3}}}=\frac{1}{s}
$$

In addition, if one scales the length of the beam while maintaining a constant width and height, the natural frequency can be scaled more aggressively:

$$
\omega_{0}=\sqrt{\frac{k}{m}}=\sqrt{\frac{E w h^{3}}{4 l^{3} l w h}} \propto \frac{1}{s^{2}}
$$

The quality factor, or $\mathrm{Q}$ factor of the resonant beam is directly proportional to the minimum detectable tip-sample interaction force. Neglecting sources of damping, the $Q$ factor scales as:

$$
Q=\frac{\sqrt{m k}}{D} \propto \sqrt{\frac{E w h^{3} l w h}{4 l^{3}}}=\sqrt{\frac{s^{7}}{s^{3}}}=s^{2}
$$


Equation (10.15) is an example of a penalty that is incurred with downward scaling. It can be shown that for a cantilever beam, the damping value does not scale when all dimensions are scaled [193]. This statement does not hold true for all geometries. 


\section{Appendix B Modeling of Electrothermal Actuators}

There is a spectrum of modeling approaches for electrothermal actuators at the micrometer scale that includes analytical solutions to the constitutive equations; coarse, compact and intuitive lumped modeling strategies; and large quantitative finite element modeling strategies. Lumped element models (LEM's) elucidate the behaviour of devices as a function of various parameters, and are useful in guiding and optimizing the designs since they can be solved rapidly. Finite element models provide quantitative information in the electrical, mechanical and thermal domains. The following sections provide a review of the methods that have been used by various authors to capture the behaviour of electrothermal actuators.

\section{B.1 Electrical Domain}

In the electrical domain, circuit models are solved with nodal and mesh analysis techniques [194] to determine the power dissipated in a resistive heater through joule heating. Polysilicon is the material used for resistors in CMOS processes, and its resistivity is both temperature and strain dependent. The change in resistance of the heater introduces nonlinearities to the transfer function of the system. The

temperature dependence of the resistor can be modeled as feedback from the thermal domain [195], or using an iterative FEA approach [174]. The electrical power dissipated in the resistor is applied as a heat source in the thermal domain:

$$
P_{\text {electrical }}=\frac{V_{r}^{2}}{R+\Delta R\left(V_{r}\right)}=q
$$

where $V_{r}$ is the voltage across the resistor. Another expression for the electrical power that is converted into heat can be obtained by considering the current in the region of interest.

$$
P_{\text {electrical }}=I_{r}^{2}\left(R+\Delta R\left(V_{r}\right)\right)^{2}=q
$$

where $I_{r}$ is the current through the resistor. The dependence of the resistor's value on the input voltage or current is captured in the expression $\Delta R\left(V_{r}\right)$, which is a polynomial that can be extracted from measurements.

\section{B.2 Thermal Domain}

In the thermal domain, the heat equation is solved for a particular geometry to uncover the temperature distribution $T(x, y, z, t)$ in the device. 


\section{B.1.2 Analytical Methods}

The law of conservation of energy applied to heat results in a continuity equation of the form:

$$
\frac{d \tilde{Q}}{d t}+\nabla \cdot J_{Q}=\left.\tilde{P}\right|_{\text {sources }}
$$

where $\tilde{Q}$ represents the heat energy normalized to a unit volume, $\boldsymbol{J}_{Q}$ is the heat flux and $\left.\tilde{P}\right|_{\text {sources }}$ represents the heat generated in a unit volume. The conduction of heat is governed by Fourier's law:

$$
J_{Q}=-\kappa \nabla T
$$

where $\kappa$ is the thermal conductivity. This expression is analogous to Ohm's law written in continuum form. If we take the divergence of Fourier's law and substitute the result into the continuity equation, we obtain the heat equation. The homogeneous form of the heat equation for materials with isotropic specific heat capacity and thermal conductivity is:

$$
\frac{\partial T}{\partial t}-D\left(\frac{\partial^{2} T}{\partial x^{2}}+\frac{\partial^{2} T}{\partial y^{2}}+\frac{\partial^{2} T}{\partial z^{2}}\right)=0
$$

where the thermal diffusivity $D$ is:

$$
D=\frac{\kappa}{c_{p} \rho}
$$

and $c_{p}$ is the specific heat capacity, and $\rho$ is the mass density. This quantity can be thought of as the measure of thermal inertia. Heat moves rapidly in a substance with high thermal diffusivity because the substance conducts heat quickly relative to its heat capacity.

More generally, the heat equation in any coordinate system is:

$$
\frac{\partial T}{\partial t}-D \nabla^{2} T=0
$$

where $\nabla^{2}$ is the Laplace operator. The expression can be thought of in terms of the amount of thermal energy that is stored in an infinitesimal volume of material. Under steady state conditions, the time dependent change in temperature must equate to the divergence of the temperature field in the volume. In these equations, the amount of energy required for volumetric expansion is ignored, since the thermal expansion coefficient of most engineering materials is small. Ironically, it is this volumetric 
expansion that is used in electrothermal actuation; this is why thermal actuators have relatively low efficiency and must operate at high temperatures. This effect will be included in the discussion of mechanical modeling (B.3).

The heat equation is a linear partial differential equation with constant coefficients, and many methods exist to solve it for a variety of source and boundary conditions. In fact, the solution to the heat transfer equation is one of the oldest classical problems in the theory of parabolic partial differential equations. The first analytical solution technique for the heat equation was proposed by Fourier in 1822 [196]. The method of separation of variables was used to uncover eigenvalues and eigenfunctions associated with a particular set of boundary conditions, such that the solution could be expressed as the sum of all modes. A solution to the inhomogeneous problem can then be attained using Duhamel's Principle. One may also calculate the response to an impulse source, which is the impulse response or Green's function, and then use the convolution integral to find the response to an actual source [197]. In this work, a sinusoidal varying heat source is often used to actuate devices. The problem of the heat equation with a harmonic temperature variation can be solved using the method of separation of variables with complex variables [198]. The same problem can be solved with Laplace transforms [199] .

\section{B.2.2 Numerical Methods}

Finite element analysis (FEA) is a widely reported method in the design of MEMS electrothermal actuators [200], [201], [174], [202], [22], [203]. The procedure involves generating a 3-D model of the device based on process parameters. The geometry and material properties of the device are included in the model. The model is then "meshed," or divided into a number of discrete volume elements that are joined at nodes. The model is then represented as a set of $n$ ordinary differential equations, where $n$ is the number of nodes. The boundary conditions are then applied to the model, and the solver is used to obtain temperature values at the nodes. Most commercially available FEA solvers include electrothermal elements that accept inputs in the electrical domain to calculate internal heat generation and solve the heat equation in a discretized manner. Both transient and steady-state solutions to the temperature distribution within the model can be obtained in this manner. Nonlinear effects such as the temperature coefficient of resistivity and temperature coefficient of thermal conductivity are typically handled with an iterative approach. The temperature distribution can then be applied to a mechanical model to evaluate thermally induced strain and account for piezoresistive effects. 


\section{B.3.2 Lumped Element Methods}

Although FEA can provide quantitative solutions to multi-domain problems, it is not often used in parametric optimization of devices, since each iteration is computationally intensive and therefore time consuming. As such, several methods have been used to obtain compact models of thermal behaviour. A review of dynamic electrothermal simulation of Microsystems is provided in [204]. One method to reduce the size of a heat conduction problem is to adopt distributed $\mathrm{R}-\mathrm{C}$ networks to represent the device. The 1-D problem can be represented by a Cauer R-C ladder network, or a less intuitive Foster network. In [205], a "time-constant spectrum" is extracted from the network and is used to set values for a lumped RC model. Another widely investigated method to reduce the size of electrothermal models is called model order reduction. This approach reduces the number of equations $n$ representing the system using one of several algorithms that are described in the literature [206], [207], [208]. Borovic et. al. [203] discusses a method for extracting a state-space model from the FEA results in order to design a controller for thermal MEMS devices.

In the DC steady state, the heat equation reduces to the Poisson equation, which can be solved using a number of numerical techniques. Senturia [149] develops a finite difference lumped element model and eigenfunction methods to solve the heat equation under static boundary conditions. The methods are then both extended to include transient effects, thus solving the time-dependent heat equation. Several authors have used such lumped modeling techniques to uncover the temperature distribution in electrothermal actuators [209], [210], [195]. The RC ladder that is obtained using this technique may be treated with concepts that are borrowed from microwave transmission line theory [211]. The position and time dependent temperatures that are obtained with this method are valid up to a certain length that depends on frequency and the geometric parameters of the thermal conduction path. This approach represents a compromise between continuous and lumped element approaches.

\section{B.4.2 Thermal Boundary Conditions}

Within the thermal domain, several factors may influence the temperature distribution in the device. Pal [211] discusses the application of boundary conditions to the heat equation. The joule heat within a volume (internal heat generation) is applied as an input on the right side of the heat equation as follows:

$$
\frac{\partial T}{\partial t}-D\left(\frac{\partial T}{\partial x}+\frac{\partial T}{\partial y}+\frac{\partial T}{\partial z}\right)=\frac{1}{c_{p} \rho} q, \quad q=\frac{d Q}{d t}=P_{\text {electrical }}
$$


The substrate is usually considered as a heat sink that remains at room temperature. Thermal conduction through the device to the heat sink is modeled with the use of a constant temperature boundary condition. In many cases, the heater is localised and provides power to a structure through a cross-section. The bimorph actuators discussed in this work are heated in this fashion. The boundary condition associated with conductive heat transfer with power $p$ through a cross-sectional area $A_{p}$ with normal vector $\boldsymbol{n}$ can be expressed as:

$$
\kappa \Delta T \cdot \boldsymbol{n}=\kappa \frac{\partial T}{\partial n}=\frac{p}{A_{p}}
$$

Under vacuum conditions, only conductive heat transfer to the substrate is considered, so these are the only boundary conditions that must be applied. Vacuum measurements can be used to quantify the conductive losses, and these values can be subtracted from ambient measurements to quantify other losses. Heat loss to the ambient environment may be modeled as convection. The convective heat loss boundary condition can be expressed as:

$$
\kappa \Delta T \cdot \boldsymbol{n}=\kappa \frac{\partial T}{\partial n}=h\left(T_{a}-T\right)
$$

Where $T_{a}$ is the ambient temperature and $h$ is the convection coefficient. The value $h$ may be used to capture the total heat loss due to convection and thermal diffusion, as explained in the following discussion. Ozsun et. al. [212] verifies the dominance of conduction and the negligible effect of gravity, and hence free convection, in the case of microscale heat sources surrounded by air at atmospheric pressure. Convective thin film coefficients are utilized under a constant heat flux condition to model the diffusive heat transfer in air. A thorough review of methods to model convective heat transfer is also provided in this reference. In both [174] and [212], a temperature dependent convection coefficient is applied to some surfaces to model convection. Heat transfer through the thin air gap between the heater and substrate is modeled as conduction, since in this region the Raleigh number is small. The Raleigh number is the product of the Grashof number and the Prandtl number. The Grashof number represents the ratio of buoyancy forces to viscous forces:

$$
G r=\frac{g \alpha\left(T_{s}-T_{\infty}\right) L^{3}}{v^{2}}
$$


Where $g$ is the gravitational constant, $\alpha$ is the thermal expansion coefficient, $L$ is the characteristic length, and $v$ is the kinematic viscosity of the fluid. A large Grashoff number indicates an increase in convection. The Prandtl number represents the ratio of the momentum and thermal diffusivities:

$$
\operatorname{Pr}=\frac{v}{D}
$$

A small Prandtl number indicates that heat diffuses faster than momentum, and thus thermal diffusivity dominates over momentum diffusivity. The Raleigh number is therefore:

$$
\operatorname{Ra}=\operatorname{GrPr}=\frac{g \alpha\left(T_{s}-T_{\infty}\right) L^{3}}{v D}
$$

If the Raleigh number is smaller than a critical value for a given fluid, the dominant heat transfer is conduction. In microscale systems, this value can range from $10^{-9}$ to $10^{-3}$, justifying the use of a boundary condition similar to thermal conduction at the surfaces of microscale actuators.

Once the temperature distribution is known, it is applied as an input to the mechanical domain.

\section{B.3 Mechanical Domain}

Thermal quantities are coupled into the mechanical domain through the phenomenon of thermoelasticity, which describes how materials tend to expand with increasing temperature. The work done by thermal expansion is the source of energy in electrothermal actuators.

\section{B.1.3 Analytical Methods}

Mechanical forces that arise from thermal effects have their origin in a coupling term that was neglected in the heat equation (3.5). The modified form of the equation that includes a thermoelastic term is [213]:

$$
\frac{\partial T}{\partial t}-D \nabla^{2} T+\alpha_{T}(3 \lambda+2 \mu) T_{0} \frac{\partial\left(\epsilon_{11}+\epsilon_{22}+\epsilon_{33}\right)}{\partial t}=0
$$

Here, $\alpha_{T}$ is the linear thermal expansion coefficient (TEC) and $\lambda$ and $\mu$ are the Lamé constants. This can be expressed in more familiar terms using the relations for Young's Modulus: 


$$
E=\frac{\mu(2 \mu+3 \lambda)}{\lambda+\mu}
$$

And for the Poisson's Ratio:

$$
v=\frac{\lambda}{2(\lambda+\mu)}
$$

Then, equation (3.12) can be rewritten:

$$
\frac{\partial T}{\partial t}-D \nabla^{2} T+\alpha_{T} K T_{0} \frac{\partial\left(\epsilon_{11}+\epsilon_{22}+\epsilon_{33}\right)}{\partial t}=0
$$

Here, $K$ is the bulk modulus, or modulus of volume expansion defined by:

$$
K=\frac{E}{3(1-2 v)}
$$

The bulk modulus is the analog of Young's modulus for an object subjected to identical normal stresses on all sides. When the Poisson ratio approaches 0.5 the bulk modulus approaches infinity, meaning the material is incompressible.

To summarize equations (3.12)-(3.16), some of the energy from the thermal domain is captured by the volumetric expansion term. Since the strain energy can be expressed in terms of the bulk modulus times the strain from volumetric expansion, one takes the time derivative of strain energy to obtain the term.

The linear TEC is defined as:

$$
\alpha_{T}=\frac{d \epsilon_{x}}{d T}
$$

where $\epsilon_{x}$ is the uniaxial strain. The uniaxial strain as a function of temperature can be expressed as:

$$
\epsilon_{x}(T)=\epsilon_{x}\left(T_{0}\right)+\alpha_{T} \Delta T
$$

The volume expansion coefficient of a material can be obtained by allowing it to expand in all three dimensions: 


$$
\frac{\Delta \mathrm{V}}{V}=\left(1+\epsilon_{x}\right)\left(1+\epsilon_{y}\right)\left(1+\epsilon_{z}\right)-1
$$

which leads to the expression:

$$
\frac{\Delta \mathrm{V}}{V}=3 \alpha_{T} \Delta T
$$

Single-material electrothermal actuators such as chevron-type actuators and pseudo-bimorph actuators operate on the principle of volumetric expansion. In [214], the authors solve the Euler-Bernoulli beam equation with applied strain to calculate the deflection of a chevron actuator. In [215], the linear expansion of beams in a pseudo-bimorph actuator is used to estimate its deflection assuming pure bending conditions.

In bimorph beams that comprise 2 materials with different TEC's, a moment arises from the difference in strain between the layers. This moment curls the beam towards the layer with the lower TEC. In 1925, Timoshenko [216] presented the analysis for such a structure with a constant temperature distribution. The analysis of multimorph beams experiencing strain from thermal expansion was presented in [217] and [218].

The Euler-Bernoulli beam equation provides a means for calculating the deflection characteristics of beams. For materials with uniform cross-section and constant stiffness, it can be expressed as:

$$
E I \frac{d^{4} w}{d x^{4}}=q(x)
$$

Where $w(x)$ is the transverse deflection at position $x$ along the length of the beam. The second moment of area $I$ of the beam is calculated from the dimensions of the beam:

$$
I=\iint z^{2} d y d z
$$

and the load $q(x)$ is distributed evenly along the length of the beam. The equation can be solved using a wide variety of methods, some of which are discussed in [149]. In this work, the load on the beam is rarely an evenly distributed load. To understand the application of various loading conditions to the beam equation, we integrate it successively. 


$$
\int E I \frac{d^{4} w}{d x^{4}}=E I \frac{d^{3} w}{d x^{3}}=Q
$$

$Q$ represents the shear force in the beam (internal shear force). Integrating again, we have:

$$
\int E I \frac{d^{3} w}{d x^{3}}=E I \frac{d^{2} w}{d x^{2}}=M
$$

$M$ is the bending moment in the beam (internal bending moment). This term is of fundamental importance in both the bimorph and single material actuators, since it is the mechanism by which deflection is achieved. It is interesting to note that for a bimorph beam, the moment is generated by the difference in TEC's between the constituent materials. This moment is calculated from the surfaces of the beam to its neutral axis, which is a small length for slender beams. For single material actuators like the chevron actuator, the force arises from the thermal expansion of a single material, and the moment comes from reaction forces at the anchor points. It is therefore not surprising that the workper-unit-volume of chevron actuators is higher than that of bimorphs made of the same "working material". The term "working material" refers to the material with the higher TEC in the bimorph, even though the actual work is proportional to the difference in TEC's between the two materials that are in use.

From calculus, it is known that if the slope $\frac{d w}{d x}$ of a beam is small, then

$$
\frac{1}{\rho}=\frac{\frac{d^{2} w}{d x^{2}}}{\left(1+\left(\frac{d w}{d x}\right)^{2}\right)^{\frac{3}{2}}} \approx \frac{d^{2} w}{d x^{2}}
$$

Where $\rho$ is the radius of curvature of the beam. In most of the literature on bimorph actuators, it is assumed that this value is constant throughout the beam. This is a convenient assumption since it allows for the straightforward calculation of the $x$ and $y$ coordinates at any point in the beam. Some authors have considered temperature gradients that cause a non-uniform $\rho$ [219], [150]. However, in these works only the angle of deflection is calculated from the non-uniform moment. If we integrate the beam equation once again, we have:

$$
\int E I \frac{d^{2} w}{d x^{2}}=E I \frac{d w}{d x}=E I \tan \theta \approx E I \theta
$$


This term is proportional to the slope of the beam. For small deflections, it is assumed that the angle of the beam is equal to the slope. In all cases, when we apply an external load on the beam, whether it is a shear force, a bending moment, or an angular deflection, the sign of the corresponding term is reversed.

\section{B.2.3 Numerical Methods}

Solutions to the beam equation can also be obtained for more complex loading conditions, using the Principle of Virtual Work [149], which states that in static equilibrium, the total work done by external forces in creating a deformation must equal the total internal stored energy in the deformed state. Variational methods can then be used to minimize the difference between these quantities in order to solve for the beam's deflection. This is the basis for FEA methods for a meshed structure, in which the variational parameters are the nodal displacements of the mesh elements.

\section{B.3.3 Lumped Element Models}

For static problems, the Euler-Bernoulli equation provides all the intuition required to optimize various beam parameters under constraints of deflection, stiffness, or strain. The deflection of an actuator sets the scan range that can be attained. The stiffness of a beam influences its response to tip-sample interaction forces. The strain in a beam can be used to capture externally applied forces and deflections through an integrated piezoresistor. These quantities may be implemented in a lumped element form using displacement as the effort variable and velocity as the flow variable. Such a static model can be imported into the electrical domain by replacing the mechanical displacement with voltage, the velocity with current, and the compliance with capacitance. Linear expressions can then be used to extract quantities like the maximum strain.

\section{B.4.3 Time-Dependence of the Beam Equation}

The time-dependent deflection of a beam is important in this work. In dynamic AFM, the sensitivity of a piezoresistor is enhanced by placing it in mechanically resonant structure. On the other hand, undesirable vibrations in the flexural suspensions of scanners may be excited by external vibrations, or by the integrated actuators. Analytical methods to calculate the dynamical quantities of a vibrating beam are the subject of numerous texts [220], [221]. In [222], the authors provide a historical perspective and describe the dynamics of transversely vibrating beams using four engineering theories. The dynamic Euler-Bernoulli beam equation is derived from Hamilton's principle. The Lagrangian is: 


$$
\mathcal{L}:=\frac{1}{2} \mu\left(\frac{\partial w}{\partial t}\right)^{2}-\frac{1}{2} E I\left(\frac{\partial^{2} w}{\partial x^{2}}\right)^{2}+q(x) w(x, t) \equiv \mathcal{L}\left(x, t, w, \frac{\partial w}{\partial t}, \frac{\partial^{2} w}{\partial x^{2}}\right)
$$

In this equation, the sum of the kinetic energy (first term), potential energy stored as strain (second term), and work done by external forces (third term) is to be minimised. The corresponding EulerLagrange equation is:

$$
\frac{\partial \mathcal{L}}{\partial w}-\frac{\partial}{\partial t}\left(\frac{\partial \mathcal{L}}{\partial \dot{w}}\right)+\frac{\partial^{2}}{\partial x^{2}}\left(\frac{\partial \mathcal{L}}{\partial w_{x x}}\right)=0
$$

Substituting into (3.22), we obtain:

$$
E I\left(\frac{\partial^{4} w}{\partial x^{4}}\right)=-\mu \frac{\partial^{2} w}{\partial t^{2}}+q(x)
$$

This expression is valid for beams with a constant cross section and Young's Modulus. The homogeneous solution of this equation can be obtained with the same methods that are used to solve the heat equation. A simple method to obtain the resonant frequencies is to use the Raleigh-Ritz Method as described in [149]. At resonance, the kinetic energy and potential energy of the beam are equal. The kinetic energy of a mode can be estimated by assuming a trial function that matches the expected shape of the mode. The potential energy can be obtained by directly calculating the strain energy stored in the particular shape. This value is equal to the kinetic energy, which may be expressed in terms of frequency.

Lumped element models can capture dynamic effects in beams through the use of properly chosen values for the mass, stiffness, and damping terms. For a given mode of vibration with no damping, the resonant frequency is given by

$$
\omega_{0}=\sqrt{\frac{k}{m}}
$$

The Principle of Virtual Work can be used to obtain the spring constant $k$ for a desired mode shape, and the lumped mass corresponding to this mode can then be obtained with

$$
m=\frac{k}{\omega_{0}^{2}}
$$


The dynamical model can then be represented in the electrical domain using the same substitution as the static model (capacitors replace compliances), but with the addition of an inductor to represent the mass, and a resistor to represent damping. If multiple modes are of interest, each quantity must be calculated for each of the modes, and the models can be connected in parallel to the forcing sources. Since by definition, all modes are orthogonal, the Principle of Superposition can be invoked to find the sum of these modes to evaluate the overall response of the system. 


\section{Appendix C Derivation of Lumped Parameter Component Values}

\section{C.1 Thermal Model of Lateral Actuator}

The thermal model for a lateral actuator must capture the temperature distribution along the length of the beam. One reason for this requirement is that the temperature gradient has an effect on the displacement, even when the average temperature is the same, as shown in Figure 4. Another reason is that the temperature profile of the beam is frequency dependent. The complete 1D analytical expression for the time- and position-dependent heat problem for this case may be obtained by solving the partial differential equation using standard methods [199]. To uncover the temperature variation in the cross section of the beam, a harmonically varying temperature is applied at the interface between the two materials in the middle of the beam and the surface of the beams is considered to be insulated. For the axial temperature distribution problem, the heat source is represented as a harmonically varying Neumann boundary condition on one side of the beam, while the other side of the beam is considered insulated (fixed Neumann boundary condition).

Several observations can be made from the solution to the heat problem[150]. First of all, the solution takes the form of a dissipating thermal wave with a propagation constant and penetration depth. As the frequency increases, the penetration depth decreases. We denote the turnover frequency $f_{t}$ as the frequency at which the thermal wave does not reach the end of the beam. At this frequency, the beam is considered "thermally long." The constriction of the temperature distribution is the reason that thermal actuators have a limited bandwidth, beyond which the amplitude rolls off like a single-pole RC transfer function. A second observation is that the phase of the temperature oscillations varies along the length of the beam. As the frequency is increased, the phase along the length of the beam may vary by 180 degrees, meaning that the moment applied at the base of the beam is opposite in phase when compared to the smaller moment applied at the tip. This detracts further from the attainable deflection at higher frequencies. A third observation is that the amplitude of the temperature variation near the tip of the beam is much smaller than at its base. This intuitive result implies that sensors prone to thermal disturbances should be located far away from the heaters, especially when heaters are operated at a frequency for which the beam is thermally long.

The development of a lumped element model that captures thermal effects begins with the 1-D heat equation: 


$$
\frac{\partial T}{\partial t}-D \frac{\partial^{2} T}{\partial x^{2}}=\left(T_{a}-T\right) h_{c o n v} w
$$

The left side of this equation is a simplified expression for the heat equation (10.20) in 1 dimension, where it is assumed that the temperature is constant over the cross section of the beam. The right side represents a temperature-dependent sink term to capture convection to the ambient environment (at temperature $T_{a}$ ) with a convection coefficient $h_{\text {conv }}$ over the constant-temperature area $w \partial x$. Since the lumped elements are capacitors and resistors, the values of these elements should correspond to the material properties of heat capacity and thermal conductivity. Substituting equation (10.21) into equation (10.49) gives:

$$
\rho c_{p} \frac{\partial T}{\partial t}-\kappa \frac{\partial^{2} T}{\partial x^{2}}=\rho c_{p} w h_{c o n v}\left(T_{a}-T\right)
$$

This expression can be applied to a beam of constant cross-section with isotropic thermal conductivity $\kappa$, density $\rho$, and heat capacity $c_{p}$. The geometry of the beam was shown in Figure 7 . The finite difference approximation for the Laplacian term in equation (10.50) is:

$$
\kappa \frac{d^{2} T}{d x^{2}} \cong \kappa w h \frac{T_{1}+T_{3}-2 T_{2}}{(\Delta x)^{2}}
$$

Using the fact that the cross section of the beam is constant, and rearranging the terms in (10.51), one obtains:

$$
\kappa w h \frac{T_{1}+T_{3}-2 T_{2}}{(\Delta x)^{2}}=\frac{T_{1}-T_{2}}{(\Delta x)^{2} / \kappa A}+\frac{T_{3}-T_{2}}{(\Delta x)^{2} / \kappa A}
$$

where $A$ is the cross sectional area of the beam. The convection term may also be included in the finite difference approximation. Since equation (10.51) is a second order central difference approximation, the convection term may be applied to its center node as a current with a value of:

$$
2 \rho c_{p} w h_{\text {conv }}\left(T_{a}-T\right) \cong i_{c o n v}=2 \rho c_{p} w h_{\text {conv }}\left(T_{a}-T_{2}\right)
$$

where the factor of 2 accounts for the fact that convection is occurring over the surface area of both elements. The finite difference approximation of equation (10.50) can now be expressed using (10.51)(10.53) as: 


$$
2 A \rho c_{p} \frac{d T}{d t}-\left[\frac{T_{1}-T_{2}}{(\Delta x)^{2} / \kappa A}+\frac{T_{3}-T_{2}}{(\Delta x)^{2} / \kappa A}\right]=2 \rho c_{p} w h_{c o n v}\left(T_{a}-T_{2}\right)
$$

where the material properties have been grouped with the dimensional and time dependent quantities for convenience. Multiplying equation (10.54) by $\Delta x$ and rearranging results in an expression that resembles a Kirchoff's Current Law nodal equation:

$$
2 A \rho c_{p}(\Delta x) \frac{d T}{d t}+\left[\frac{T_{2}-T_{1}}{(\Delta x) / \kappa A}+\frac{T_{2}-T_{3}}{(\Delta x) / \kappa A}\right]+2 \rho c_{p} w h_{c o n v}\left(T_{2}-T_{a}\right) \Delta x=0
$$

Each term in this expression represents a current entering the central node of the circuit in Figure 8: Thermal Domain Representation of a Lateral Electrothermal Actuator. As discussed, in the thermal convention, temperature is the across variable and current is the through variable. The first term captures the time dependence of the temperature, which is implemented by the current through a thermal capacitor of value:

$$
C_{t h}=2 A \rho c_{p} \Delta x
$$

The second term captures the effect of the series thermal conductivity between nodes. The value of a lumped resistor between the nodes can be extracted by noting that the terms represent branch currents:

$$
i_{12}=\frac{T_{2}-T_{1}}{R_{12}}=\frac{T_{2}-T_{1}}{(\Delta x) / \kappa A}, \quad i_{32}=\frac{T_{2}-T_{3}}{R_{23}}=\frac{T_{2}-T_{3}}{(\Delta x) / \kappa A}
$$

Because the two elements in Figure 8: Thermal Domain Representation of a Lateral Electrothermal Actuator are identical, the resistances are equal:

$$
R_{12}=R_{23}=R_{\text {Cond }}=(\Delta x) / \kappa A
$$

Because the cross section of the beam consists of two materials, the thermal conductivity $\kappa$ is calculated as the area-weighted average of all the members in the beam. In this calculation, the beam is discretized into single material members with boundaries defined by the transitions between different materials. Upon inspection of the cross section in Figure 7, there are 4 metal layers, each with a $\mathrm{SiO}_{2}$ layer underneath it, so the model is separated into 8 sections in the z-direction. 3 of the metal layers span only a partial width of the beam. These layers are further discretised in the y-direction. Thus, the total number of members is 11 . 


$$
\kappa=\frac{1}{w_{a} t_{a}} \sum_{\text {members }} \kappa_{i} \Delta \mathrm{y}_{i} \Delta \mathrm{z}_{i}
$$

Similar expressions are used to find the effective density and specific heat capacity:

$$
\rho=\frac{1}{w_{a} t_{a}} \sum_{\text {members }} \rho_{i} \Delta \mathrm{y}_{i} \Delta \mathrm{z}_{i}, \quad c_{p}=\frac{1}{w_{a} t_{a}} \sum_{\text {members }} c_{p_{i}} \Delta \mathrm{y}_{i} \Delta \mathrm{z}_{i}
$$

The value of the thermal resistor that accounts for convection from the 2 elements is similarly extracted from equation (10.55):

$$
R_{\text {conv }}=\frac{T_{a}-T_{2}}{i_{c o n v}}=\frac{1}{2 w \rho c_{p} h_{c o n v} \Delta x}
$$

Thermal Domain Representation of a Lateral ET actuator

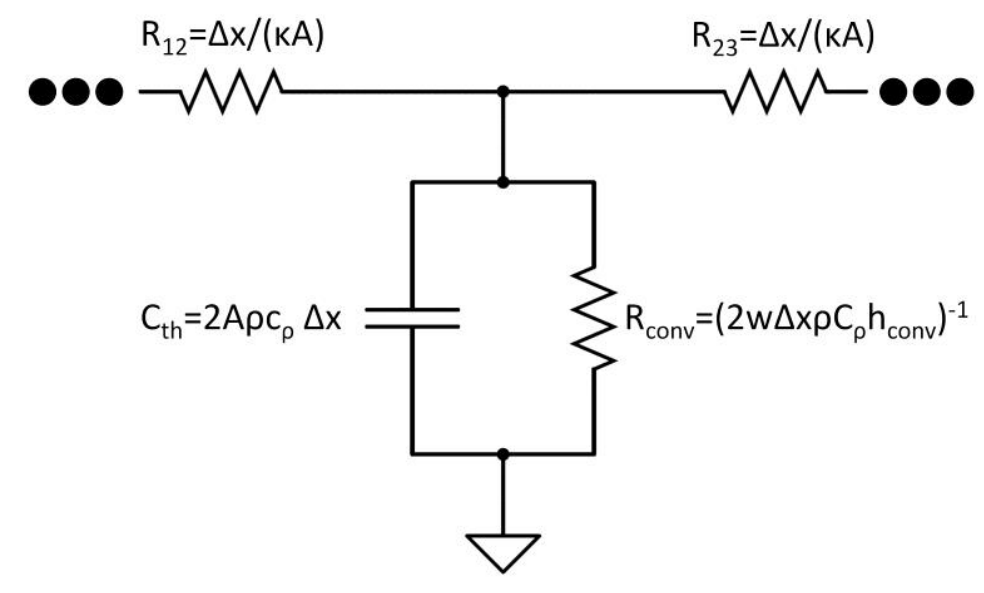

One section of the thermal model shown in the figure above and does not include any heat sources, since the self-heated resistors are placed in discrete locations outside of the bimorph beams. The heaters cannot be integrated within the slender actuator beams for several reasons. The width of the beams must be minimized in order to achieve appreciable deflections from the bending moments. Such narrow beams do not provide enough sidewall protection for the polysilicon resistors, which would be exposed and etched away during the silicon etch step of the release process. In addition, long thin resistors with large resistance values would require voltages that exceed the CMOS process specifications. Furthermore, the piezoresistivity of the material would contribute additional nonlinearities upon deformation. In fact, the combination of thermal and piezoresistive effects in 
slender beams has been exploited to create self-sustaining mechanical oscillators. [151] Vertical actuators do not need to be narrow, since the bending moments generate deflections in the out-ofplane direction. Thus, a resistor embedded within the actuator would have sufficient sidewall protection and could possess a reasonable resistance value. Nevertheless, such a configuration would still suffer from piezoresistive nonlinearities.

\section{C.2 Mechanical Model of Lateral Electrothermal Actuators}

The dynamic temperature distribution from the thermal model is coupled to the mechanical domain in order to calculate bending moments and deflection. The thermal dynamics are responsible for the lowfrequency roll-off that is observed in the transfer functions of these components. The actuators are intended to operate well below their mechanical resonant frequency to provide quasi-static displacements, so the dynamics in the mechanical domain are not considered in the model. For static mechanical analysis, the model of a lateral actuator consists of a voltage source representing the moment from thermal expansion, and a spring (capacitor) that stores potential energy.

The static Euler-Bernoulli beam equation is used to calculate the deflection in a beam under a constant moment load. Equation (10.41) and (10.42) may be rearranged to relate the deflection of the tip to the applied moment:

$$
\frac{d^{2} y}{d x^{2}} \approx \frac{1}{\rho}=\frac{M}{E I}
$$

where the variable $y$ is used to indicate the direction of deflection. Integrating equation (10.62) twice yields expressions for the tip angle and deflection:

$$
\begin{gathered}
\int \frac{d^{2} y}{d x^{2}}=\frac{d y}{d x} \approx \frac{x}{\rho}=\theta=\frac{M x}{E I} \\
\int \frac{d y}{d x}=y(x) \approx \frac{M x^{2}}{2 E I}
\end{gathered}
$$

The quantities that need to be identified in the beam are therefore the Young's modulus $E$ and the moment of inertia $I$, whose product $E I$ is the flexural rigidity, or the effective stiffness of the beam to an applied moment. The reader is reminded that a small angle approximation was made in order to relate the deflection $w$ to the moment; no such approximation was made to relate the radius of curvature $\rho$ to the moment. The beam in a multimorph actuator is not homogeneous, so an effective Young's modulus 
and the moment of inertia must be calculated. A cross section and 3D rendering of a single beam in a multimorph lateral actuator were shown in Figure 7. First, the position of the neutral axis is calculated. Then, the effective stiffness of the beam is calculated. Finally, the moment is expressed as a function of temperature. These calculations follow the procedure outlined in [218].

The placement of the neutral axis can be calculated by taking the modulus-weighted centroid of the beam with respect to the origin:

$$
\int_{-z_{c}}^{t_{a}-z_{c}} \int_{-y_{c}}^{w_{a}-y_{c}} E y d y d z=0
$$

where $(y, z)$ are the coordinates with respect to the neutral axis. By performing a coordinate transformation $y=y_{b}-y_{c}$, the distance of the neutral axis from the beam origin can be found in terms of the material properties and beam geometry using

$$
y_{c}=\frac{\sum_{\text {members }} \int_{z_{b, i}}^{z_{b, i}+\Delta z_{i}} \int_{y_{b, i}}^{y_{b, i}+\Delta y_{i}} E_{i} y_{b, i} d y_{b} d z_{b}}{\sum_{\text {members }} \int_{z_{b, i}}^{z_{b, i}+\Delta z_{i}} \int_{y_{b, i}}^{y_{b, i}+\Delta y_{i}} E_{i} d y_{b} d z_{b}}
$$

where $E_{\mathrm{i}}$ is the biaxial modulus of the $\mathrm{i}^{\text {th }}$ member for bending under the action of thermal or residual stresses. $\left(y_{\mathrm{b}, \mathrm{i}}, z_{\mathrm{b}, \mathrm{i}}\right)$ is the coordinate of the $\mathrm{i}^{\text {th }}$ member with respect to the beam origin, and $\Delta y_{\mathrm{b}, \mathrm{i}}$ and $\Delta z_{\mathrm{b}, \mathrm{i}}$ are the member width and thickness, respectively. An analogous equation exists for the distance of the neutral axis from the beam origin in the $z$ direction. When the beam undergoes pure bending, the length of the neutral axis remains constant, while fibers that are located on either side of the neutral axis experience strains in opposite directions. It is therefore intuitive that in a properly designed multimorph actuator, materials with different TEC's should be confined to either side of the neutral axis. Details on the variation of the neutral axis position with the internal metal width ratio are discussed in [218]. The remainder of this discussion assumes that the neutral axis position is chosen to coincide with the boundary between different materials in the beam.

The flexural rigidity of the multimorph is given by:

$$
(E I)_{e f f, z}=\sum_{m e m b e r s} \int_{z_{i}}^{z_{i}+\Delta z_{i}} \int_{y_{i}}^{y_{i}+\Delta y_{i}} E_{i}(y) y^{2} d y d z
$$

The moment term about the z-axis arises from process-related in-plane film stress (residual stress) and temperature-dependent thermal stress. The residual stress contribution is given by: 


$$
M_{z}=\sum_{\text {members }} \int_{z_{i}}^{z_{i}+\Delta z_{i}} \int_{y_{i}}^{y_{i}+\Delta y_{i}} \sigma_{i}(y) y d y d z
$$

In between the metal layers, the $\mathrm{SiO}_{2}$ material spans the width of the beam. In addition, the neutral axis falls close to the center of the beam in the y direction, because the Young's Modulus of $\mathrm{Al}$ and $\mathrm{SiO}_{2}$ are similar. Therefore, it is reasonable to neglect the moment arising from these intermetal dielectric layers, and consider instead the contributions from the layers containing metal, which are the "driving layers." For a beam with $N$ driving layers, the moment from residual stress is:

$$
M_{z, r e s}=N t_{m}\left[\sigma_{m} \int_{-y_{c}}^{w_{m}-y_{c}} y d y+\sigma_{o} \int_{w_{m}-y_{c}}^{w_{a}-y_{c}} y d y\right]
$$

Since the metal width is set to $w_{m}=y_{c}$, the above expression evaluates to:

$$
M_{z, r e s}=\frac{N t_{m}}{2}\left[\sigma_{o}\left(w_{a}-w_{m}\right)^{2}-\sigma_{m} w_{m}^{2}\right]
$$

This substitution ensures that the source terms in the models may be expressed explicitly in terms of the relevant quantities (e.g. temperature in the thermal domain), while the lumped elements are expressed in terms of geometric parameters and material properties that may be consolidated. The thermal stress that results from an increase in temperature is:

$$
\sigma_{t h}=\alpha E \Delta T
$$

This stress term may be substituted into equation (10.70), replacing the residual stress terms with thermal stress terms to obtain an expression for the thermally induced moment:

$$
M_{z, t h}=\frac{N t_{m}}{2}\left[\alpha_{o} E_{o}\left(w_{a}-w_{m}\right)^{2}-\alpha_{m} E_{m} w_{m}^{2}\right] \Delta T
$$

The bracketed term on the right may be thought of as a thermal moment scaling factor. This term and the constant parameters may be combined for compactness into:

$$
S_{M_{-} z, t h}=\frac{N t_{m}}{2}\left[\alpha_{o} E_{o}\left(w_{a}-w_{m}\right)^{2}-\alpha_{m} E_{m} w_{m}^{2}\right]
$$

In practise, the temperature value may be directly connected to the dependent voltage source, which has a gain of $S_{M_{-} z, t h}$. The tip angle may be calculated using equation (10.63) with the effective stiffness from (10.67) and the moment expressions from (10.70) and (10.72): 


$$
\begin{gathered}
\theta_{\text {res }}(L)=\frac{L_{a} M_{z, r e s}}{(E I)_{e f f}} \\
\theta_{t h}(L)=\frac{L_{a} S_{M_{-} z, t h}}{(E I)_{e f f}} \Delta T
\end{gathered}
$$

The deflection of the beam is at its tip is obtained by substituting the moment expressions from equations (10.70) and (10.72) and the effective stiffness from equation (10.67) into equation (10.64), evaluated at $\mathrm{y}=\mathrm{L}$ :

$$
\begin{gathered}
y_{r e s}(L)=\frac{M_{z, r e s} L_{a}^{2}}{2(E I)_{e f f}} \\
y_{t h}(L)=\frac{L_{a}^{2} S_{M_{-}, t h}}{2(E I)_{e f f}} \Delta T
\end{gathered}
$$

The mechanical lumped element model that couples temperature and residual stress to deflection is implemented on the basis of equations (10.76) and (10.77). A voltage source supplies the across variable, which gives rise to a current (through variable) that is stored as charge on a capacitor. The mechanical potential energy stored in the deflected beam is therefore represented by the charge stored in the capacitor. The following analogy between a rotary spring and a capacitor may be used:

$$
M=(E I) \theta \quad \leftrightarrow \quad V=\frac{1}{C} q
$$

The voltage source representing the thermally generated moment has a value of

$$
V_{t h, \theta}=S_{M_{-} z, t h} \Delta T
$$

A separate independent voltage source accounts for the moment from residual stress:

$$
V_{\text {res }, \theta}=M_{z, r e s}
$$

The capacitor has a value of: 


$$
C=\frac{1}{k}=\frac{L_{a}}{(E I)_{e f f, z}}
$$

The model in the figure below represents the tip angle in terms of the charge stored on the capacitor $\mathrm{C}_{\mathrm{m} \theta}$.

Mechanical Domain Representation of a Lateral ET actuator

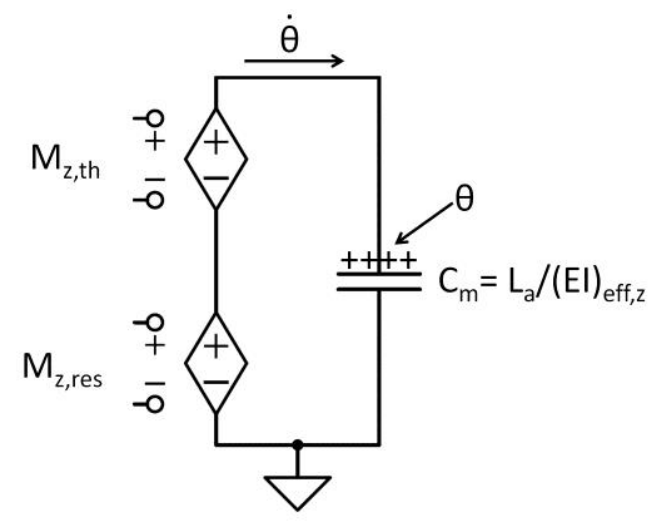

To obtain the deflection of the beam, the tip angle was integrated as indicated by equation (10.64). A new voltage source and capacitor are required to capture the tip deflection. Since the force exerted by (or on) the actuator is at the tip, it is desirable to choose a spring constant and force source that reflect the end-point load geometry. It is tempting to assume that the spring constant of an end-loaded cantilever may be used to set the value of the capacitor, and since the deflection is already known from equations (10.76) and (10.77), the value of the voltage source could be calculated using (10.78). However, this approach would not accurately represent the work done by the actuator; in fact, it would underestimate blocked force that is produced. In other words, care must be taken to preserve the potential energy in the beam so that the work done by the actuator in bending is properly transferred to the tip-referred loading condition. Energy methods like Castigliano's Method are therefore useful to calculate the value of $\mathrm{C}_{\mathrm{my}}$. The bending strain is:

$$
\epsilon=\frac{y}{\rho}=y \frac{\left(M_{z, t h}+M_{z, r e s}\right)}{E I}
$$

The total strain energy is found from:

$$
U=\frac{E W}{2} \int_{0}^{L} \int_{-\frac{W}{2}}^{\frac{W}{2}} \epsilon^{2} d y d z
$$


Neglecting the width and dropping subscripts for the generalized case, for a beam under pure bending with a constant moment:

$$
U=\frac{M^{2} L}{2 E I}
$$

The total strain energy is equated to the potential energy stored in the tip-loaded beam to obtain the effective spring constant:

$$
\frac{M^{2} L}{2 E I}=\frac{1}{2} k y^{2}
$$

The assumption here is that the cantilever behaves as a linear spring with spring constant $k$ and substituting from (10.64) for $y(L)$ :

$$
\frac{M^{2} L}{2 E I}=\frac{1}{2} k\left(\frac{M L^{2}}{2 E I}\right)^{2}
$$

Finally, solving for k gives:

$$
k=\frac{4 E I}{L^{3}}
$$

Reintroducing subscripts for the multimorph case, the corresponding capacitance value is:

$$
C_{m y}=\frac{L^{3}}{4(E I)_{e f f, z}}
$$

The values of the voltage sources are scaled to ensure that the charge on the capacitors represents the displacement, and the force becomes tip-referred:

$$
\begin{gathered}
V_{\text {residual }}=\frac{y_{\text {residual }}}{C_{m y}}=\frac{2 M_{z, r e s}}{L} \\
V_{t h}=\frac{y_{t h}}{C_{m y}}=\frac{2 S_{M_{-} z, t h}}{L} \Delta T
\end{gathered}
$$

The model in the figure below represents the tip deflection of a lateral electrothermal actuator, assuming that the temperature remains constant along the length of the beam. 


\section{Mechanical Domain Representation of ET Deflection}

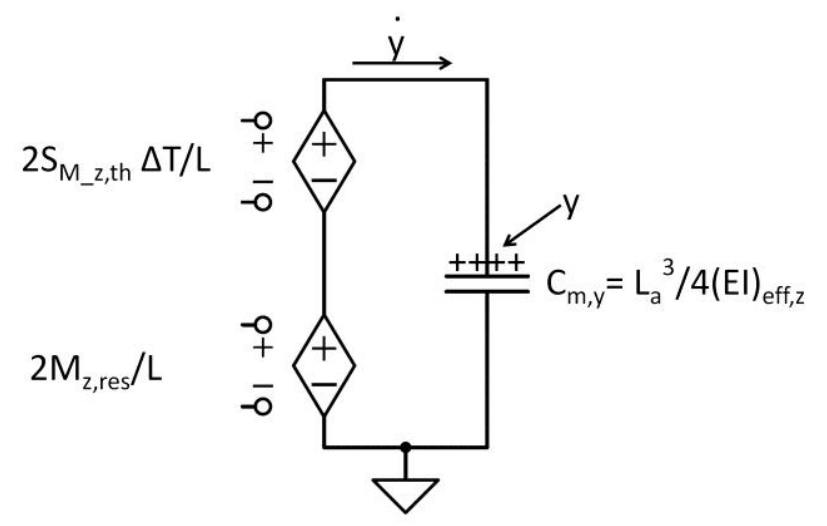

If an external force is applied to deflect the cantilever, it must be scaled in order to reflect the difference between the effective tip-referred capacitance shown here, and the intrinsic stiffness of a cantilever beam which is:

$$
C_{\text {cant }}=\frac{L^{3}}{3(E I)_{e f f, z}}
$$

The scaling factor is therefore 4:3.

Since the model only accepts one input from the thermal domain, it fails to capture the effect of the temperature gradient that was shown to exist in Figure 4, and therefore produces inaccurate estimates of the tip deflection. The average temperature, available in the center node of the circuit representaion, may be used to predict the tip angle of the beam with a single input from the thermal domain, as shown in [195]. This method cannot be applied to calculate tip deflection, since two beams with the same average temperature but different temperature profiles will produce different deflections, even though their tip angles are identical. To capture the effect of a linear thermal gradient, at least two inputs are required in the mechanical domain. The temperature gradient is then calculated, and the angle and deflection are derived for this case.

The temperature gradient between the base and tip of the actuator is simply the slope of the linear temperature distribution:

$$
m_{t h}=\frac{\Delta T_{t i p}-\Delta T_{\text {base }}}{L}
$$

The thermal moment is now a function of position: 


$$
M(x)=M_{0}\left[1+m_{t h} x\right]
$$

where the moment $M_{0}=M_{z, t h}\left(x_{0}\right)$ results from the temperature of the base of the actuator, which may be higher than room temperature. The tip angle for a multimorph with a temperature gradient is:

$$
\theta(x)=\int \frac{d^{2} y}{d x^{2}}=\int \frac{M(x)}{E I} d x=\frac{x S_{M \_y, t h} \Delta T}{E I}\left(1+\frac{m_{t h} x}{2}\right)
$$

Since the flexural rigidity is unchanged, the voltage source is simply:

$$
V_{t h, \theta}=L S_{M_{-} y, t h} \Delta T\left[1+\frac{m_{t h} L}{3 \Delta T}\right]
$$

The tip deflection is:

$$
y(x)=\int \theta(x) d x=\frac{x^{2} S_{M_{-} y, t h} \Delta T}{2 E I}\left(1+\frac{m_{t h} x}{3}\right)
$$

It was shown in Figure 4 that indeed, for beams with the same average temperature, the tip angle remains fixed while the tip deflection varies. The strain energy stored in the beam is:

$$
U=\int_{0}^{L} \frac{M(x)^{2}}{2 E I} d x=\frac{1}{2 E I} \int_{0}^{L}\left(M_{0}+M_{0} m_{t h} x\right)^{2} d x
$$

To find the tip-referred spring constant, equate the strain energy to the potential energy of the beam (treated as a linear spring) at $x=L$ from (10.96):

$$
\frac{1}{2 E I} \int_{0}^{L}\left(M_{0}+M_{0} m_{t h} x\right)^{2} d x=\frac{1}{2} k\left(\frac{x^{2} M_{0}}{2 E I}\left(1+\frac{m_{t h} x}{3}\right)\right)^{2}
$$

Solving for the effective linear spring constant $k$ and performing some algebraic simplifications,

$$
k=\frac{4 E I}{L^{3}}+\frac{4 E I m_{t h}}{3 L^{2}}-\frac{4 E I m_{t h}{ }^{3}}{3\left(L m_{t h}+3\right)^{2}}
$$

The first term on the right hand side is identical to the isothermal case, from (10.87). The second and third terms include a dependence on the temperature distribution, represented by the slope of the temperature $m_{t h}$. If the temperature gradient is removed by setting $m_{t h}=0$, the second third term vanish as expected, and the effective linear spring constant returns to the isothermal case. The value of the capacitor is: 


$$
C_{y, m_{-} t h}=\frac{1}{k}=\frac{L^{3}}{4 E I}\left[\frac{1}{3}+\frac{L m_{t h}+2}{\left(L^{2} m_{t h}^{2}+3 L m_{t h}+3\right)}\right]
$$

And the voltage source is:

$$
V_{t h, m_{-} t h}=\frac{q}{C}=2 S_{M_{-} z, t h} \frac{L^{2} m_{t h}{ }^{2}+3 L m_{t h}+3}{L\left(L m_{t h}+3\right)} \Delta T
$$

The complete quasi-static model of the electrothermal bimorph is shown below. The model accounts for thermal gradients and provides a realistic estimation of the tip-referred force and deflection by considering the strain energy produced by thermal bending moments. The quasi-static nature of the model means that it ignores the dynamics in the mechanical domain, which is why there is no lumped resistor to represent damping and no lumped capacitor to represent mass. The charge on the capacitor instantaneously follows the voltage on the source, scaled by a judiciously chosen compliance factor. Note that the resistor used to capture convection is tied directly to ground, since it is not affected by thermal isolation.

\section{Electro-thermo-mechanical Model of Deflection}

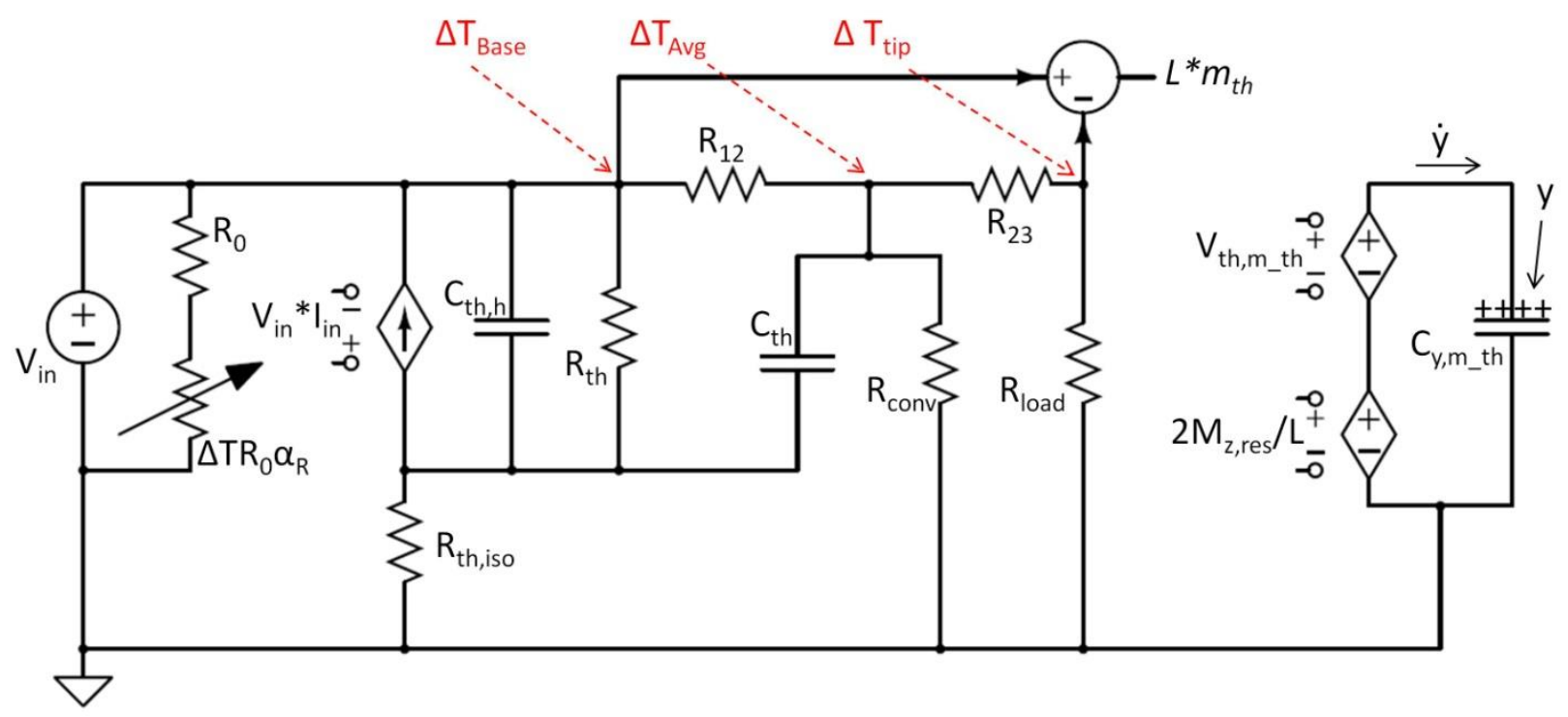




\section{C.3 Thermal Transmission Line Model}

To extract the distributed parameter model from the lumped element model, one solves Kirchoff's current and voltage laws for the lumped parameter case, then takes the limit as the length of the segment approaches zero. Applying Kirchoff's Voltage Law to the thermal model gives:

$$
v(x, t)=i(x, t) R_{t h} \Delta x+v(x+\Delta x, t)
$$

Solving Kirchoff's current law at the central node gives:

$$
i(x, t)=i(x+\Delta x, t)+v(x+\Delta x, t) G_{t h} \Delta x+\frac{\partial v(x+\Delta x, t)}{\partial t} C_{t h} \Delta x
$$

Dividing equations (10.102) and (10.103) by $\Delta x$ and taking the limit as $\Delta x \rightarrow 0$ results in the following differential equations for voltage and current:

$$
\begin{gathered}
\frac{\partial v(x, t)}{\partial x}=-i(x, t) R_{t h} \\
\frac{\partial i(x, t)}{\partial x}=-v(x, t) G_{t h}-\frac{\partial v(x, t)}{\partial t} C_{t h}
\end{gathered}
$$

Using phasor notation for the sinusoidal steady state, equations (10.104) and (10.105) simplify to:

$$
\begin{gathered}
\frac{d V(x)}{d x}=R_{t h} I(x) \\
\frac{d I(x)}{d x}=\left(G_{t h}+j \omega C_{t h}\right) V(x)
\end{gathered}
$$

These equations can be solved simultaneously to give wave equations for $V(x)$ and $I(x)$ :

$$
\begin{aligned}
& \frac{d^{2} V(x)}{d x^{2}}-\gamma^{2} V(x)=0 \\
& \frac{d^{2} I(x)}{d x^{2}}-\gamma^{2} I(x)=0
\end{aligned}
$$

where

$$
\gamma=\alpha+j \beta=\sqrt{R_{t h}\left(G_{t h}+j \omega C_{t h}\right)}
$$

The complex propagation constant $\gamma$ is a function of frequency. The real part is: 


$$
\alpha=\sqrt{\frac{\sqrt{\left(R_{t h} G_{t h}\right)^{2}+\left(\omega R_{t h} C_{t h}\right)^{2}}+R_{t h} G_{t h}}{2}}
$$

and the imaginary part is:

$$
\beta= \pm \sqrt{\frac{\sqrt{\left(R_{t h} G_{t h}\right)^{2}+\left(\omega R_{t h} C_{t h}\right)^{2}}-R_{t h} G_{t h}}{2}}
$$

The travelling wave solutions are:

$$
\begin{aligned}
& V(x)=V_{0}^{+} e^{-\gamma x}+V_{0}^{-} e^{\gamma x} \\
& I(x)=I_{0}^{+} e^{-\gamma x}+I_{0}^{-} e^{\gamma x}
\end{aligned}
$$

The current on the line may also be expressed by substituting the voltage from (10.113) into (10.104):

$$
I(x)=\frac{\gamma}{R_{t h}}\left[V_{0}^{+} e^{-\gamma x}+V_{0}^{-} e^{\gamma x}\right]
$$

The quantity relating the current to the voltage is the characteristic impedance of the line:

$$
Z_{0}=\frac{V_{0}^{+}}{I_{0}^{+}}=\frac{V_{0}^{-}}{I_{0}^{-}}=\frac{R_{t h}}{\gamma}=\sqrt{\frac{R_{t h}}{G_{t h}+j \omega C_{t h}}}
$$

Converting back to the time domain, the voltage waveform can be expressed as:

$$
v(x, t)=\left|V_{0}^{+}\right| \cos \left(\omega t-\beta x+\varphi^{+}\right) e^{-\alpha x}+\left|V_{0}^{-}\right| \cos \left(\omega t+\beta x+\varphi^{-}\right) e^{\alpha x}
$$

where $\varphi^{ \pm}$is the phase angle of the complex voltage $V_{0}^{ \pm}$. Equation (10.117) captures the exponential decay and the phase variance of the temperature along the beam. The thermal wavelength on the line is:

$$
\lambda=\frac{2 \pi}{\beta}
$$

and the phase velocity is: 


$$
v_{p}=\frac{\omega}{\beta}=\lambda f
$$

The transmission line model derived in this section may be used to determine the time and positiondependent temperature along a thermally excited beam. Consider a transmission line of length $L$ with characteristic impedance $Z_{0}$. To find the amplitude and phase at a position $x$, the transmission line may be divided into two segments, one of length $x$ and the other of length $L-x$.

The fact that there is no inductor in the thermal transmission line has an important consequence: there is no mechanism for the generation of reflected waves in this structure. It is therefore not necessary to match the source and load impedances to that of the transmission line. Instead, the source and load impedances should be minimized in order to improve heat transfer in a thermal shunt path. Of course, in the case of thermal isolation paths, the opposite is true, so that any additional impedance in series with the source and load is helpful.

To illustrate the application of the transmission line model, consider a thermal isolation path between a self-heated resistor and the tip. A heat current is present at the input, and the goal is to measure the temperature at the other end. The current leaving the tip is 0 , so equation (10.115) becomes:

$$
I(L)=\frac{\gamma}{R_{t h}}\left[V_{0}^{+} e^{-\gamma x}-V_{0}^{-} e^{\gamma x}\right]=0
$$

Thus, the ratio of forward and backward voltages can be expressed as:

$$
\frac{V_{0}^{+}}{V_{0}^{-}}=e^{2 \gamma L}
$$

The input current, which is known, can now be expressed solely in terms of the forward voltage:

$$
I(0)=\frac{\gamma}{R_{t h}}\left[V_{0}^{+} e^{-\gamma x}-V_{0}^{+} e^{-\gamma x}\right]=\frac{2 \gamma}{R_{t h}} V_{0}^{+}
$$

The voltage from equation (10.113) is:

$$
V(x)=\frac{I(0) R_{t h}}{\gamma} e^{-\gamma x}
$$

The voltage from (10.123) may be evaluated at any number of points along the beam, and its value may be applied to the corresponding nodes in the mechanical domain. 
Although microwave engineering CAD tools include transmission line elements that may be modified to suit this model, most Spice-type solvers do not include distributed parameter models. To implement the transmission line model in a Spice-like solver, it is convenient to express the characteristic impedance as a T-model, as shown below.

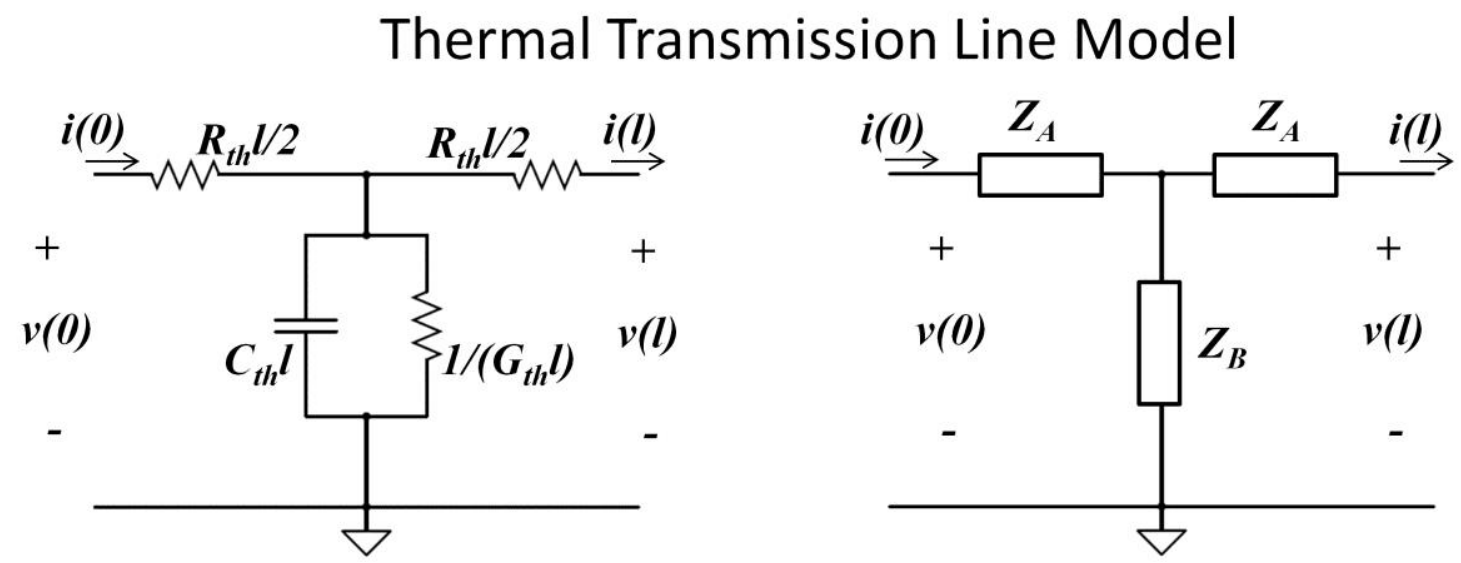

From equations (10.113) and (10.115),

$$
\frac{V(0)}{I(0)}=\frac{V_{0}^{+}+V_{0}^{-}}{\frac{\gamma}{R_{t h}}\left(V_{0}^{+}-V_{0}^{-}\right)}=Z_{A}+Z_{B}=\frac{R_{t h}}{\gamma} \frac{\left(e^{2 \gamma L}+1\right)}{\left(e^{2 \gamma L}-1\right)}
$$

Also, using (10.114), the ratio can be expressed as:

$$
Z_{A}+Z_{B}=\frac{R_{t h}}{\gamma} \frac{V_{0}^{+}+V_{0}^{-}}{\left(V_{0}^{+}-V_{0}^{-}\right)}=\frac{R_{t h}}{\gamma} \frac{\left(e^{2 \gamma L}+1\right)}{\left(e^{2 \gamma L}-1\right)}
$$

The impedances $Z_{A}$ and $Z_{B}$ form a resistive divider, such that

$$
\frac{V(L)}{V(0)}=\frac{Z_{B}}{Z_{A}+Z_{B}}=\frac{R_{t h}}{\gamma} \frac{\left(e^{2 \gamma L}+1\right)}{\left(e^{2 \gamma L}-1\right)}
$$

The voltage at the input may be expressed solely in terms of the reverse voltage:

$$
V(0)=V_{0}^{-}\left(1+e^{2 \gamma L}\right) \quad \therefore V_{0}^{-}=\frac{V(0)}{\left(1+e^{2 \gamma L}\right)}
$$

The voltage at the output may also be expressed solely in terms of the reverse voltage: 


$$
V(L)=V_{0}^{-}\left(2 e^{\gamma L}\right)
$$

Thus, the ratio from (10.126) may also be expressed as:

$$
\frac{V(L)}{V(0)}=\frac{Z_{B}}{Z_{A}+Z_{B}}=\frac{2 e^{\gamma L}}{1+e^{2 \gamma L}}
$$

The values for the impedances $Z_{A}$ and $Z_{B}$ are:

$$
\begin{gathered}
Z_{A}=\frac{R_{t h}}{\gamma} \frac{e^{\gamma L}-1}{e^{\gamma L}+1}=Z_{0} \tanh \frac{\gamma L}{2} \\
Z_{B}=\frac{R_{t h}}{\gamma} \frac{2 e^{\gamma L}}{e^{\gamma L}-1}=\frac{Z_{0}}{\sinh \gamma L}
\end{gathered}
$$

As with the model incorporating $Z_{0}$, this model can be subdivided into shorter lengths in order to apply temperatures to various points in the mechanical model.

It is possible to obtain the time and position dependent temperature in a beam under AC excitation with FEA software; however, the FEA solver used in this work requires a transient thermal boundary condition at each frequency of interest, and many time-steps would be required for the model to reach steady state so that the amplitude and phase can be measured. The utility of the distributed parameter model is underscored by its rapid convergence, enabling parametric optimization during the design process. The model also provides insight into measurements obtained with scanning thermal microscopy.

\section{C.4 Vertical Actuator Mechanical Model}

Taking coordinates with respect to the beam origin, using the coordinate transformation $\tilde{z}=z-z_{c}$, the position of the neutral axis is obtained with a simplified version (for 2 members) of equation (10.66):

$$
z_{c}=\frac{\int_{y_{o}}^{y_{o}+\Delta y} \int_{\tilde{z}_{o x}}^{\tilde{z}_{o x}+\Delta z_{o x}} E_{o x} \tilde{z} d \tilde{z} d y+\int_{y_{0}}^{y_{o}+\Delta y} \int_{\tilde{z}_{m}}^{\tilde{z}_{m}+\Delta z_{m}} E_{m} \tilde{z} d \tilde{z} d y}{\int_{y_{o}}^{y_{o}+\Delta y} \int_{\tilde{z}_{o x}}^{\tilde{z}_{o x}+\Delta z_{o x}} E_{o x} d \tilde{z} d y+\int_{y_{0}}^{y_{o}+\Delta y} \int_{\tilde{z}_{m}}^{\tilde{z}_{m}+\Delta z_{m}} E_{m} d \tilde{z} d y}
$$

The positions of the oxide and metal layers in the beam are similar to those of a single working layer in the lateral multimorph, but rotated such that the moment bends the beam downwards (towards the 
substrate). Using coordinates with respect to the neutral axis location, the effective stiffness for bending about the $y$ axis is obtained using,

$$
(E I)_{e f f, y}=\int_{y_{0}}^{y_{0}+\Delta y} \int_{z_{0}}^{z_{0}+\Delta z} E(z) z^{2} d z d y
$$

In this expression, the Young's modulus is a function of position that changes from $E_{o x}$ to $E_{m}$ at the interface between the oxide and metal. When calculating the internal bending moments of the beam, one cannot make the simplifying assumption that the position of the neutral axis is coincident with the position of the metal-oxide transition, as was done in the lateral multimorph case. The thickness of the metal and oxide layers is defined by the foundry process and cannot be adjusted by the designer as in the case of lateral actuators, wherein the width of metal layers may be adjusted in layout. Since the oxide layer below the bottom metal layer, the neutral axis falls within the oxide. The moments from residual stress and thermal stress are:

$$
\begin{array}{r}
M_{y, r e s}=\Delta y\left[\sigma_{o x} \int_{z=z_{o x}}^{0} z d z+\sigma_{o x} \int_{0}^{z=z_{o x}+\Delta z_{o x}} z d z+\sigma_{m} \int_{z=z_{o x}+\Delta z_{o x}}^{z=z_{m}} z d z\right] \\
=\Delta y\left[\frac{\sigma_{o x}}{2}\left(\Delta z_{o x}^{2}+2 z_{o x} \Delta z\right)+\frac{\sigma_{m}}{2}\left(\Delta z_{m}^{2}-z_{o x}^{2}-\Delta z_{o x}^{2}-2 z_{o x} \Delta z\right)\right] \\
M_{y, t h}=\Delta y \Delta T\left[\frac{\alpha_{o} E_{o}}{2}\left(\Delta z_{o x}^{2}+2 z_{o x} \Delta z\right)+\frac{\alpha_{m} E_{m}}{2}\left(\Delta z_{m}^{2}-z_{o x}^{2}-\Delta z_{o x}^{2}-2 z_{o x} \Delta z\right)\right]
\end{array}
$$

The bracketed term may be expressed as a moment scaling factor for compactness, and the width of the beam can be absorbed in this term, leaving the temperature variable to be expressed explicitly in subsequent calculations:

$$
S_{M_{-} y, t h}=\Delta y\left[\frac{\alpha_{o} E_{o}}{2}\left(\Delta z_{o x}^{2}+2 z_{o x} \Delta z\right)+\frac{\alpha_{m} E_{m}}{2}\left(\Delta z_{m}^{2}-z_{o x}^{2}-\Delta z_{o x}^{2}-2 z_{o x} \Delta z\right)\right]
$$

The tip angles may be calculated with:

$$
\theta_{\text {res }}=\frac{M_{y, r e s} L}{(E I)_{e f f, y}}
$$

and 


$$
\theta_{t h}=\frac{M_{y, t h} L}{(E I)_{e f f, y}}=\frac{\Delta T S_{M_{-} y, t h} L}{(E I)_{e f f, y}}
$$

Finally, the static deflections resulting from residual and thermal stress are:

$$
\begin{aligned}
& z_{r e s}(L) \approx \frac{L^{2}}{4(E I)_{e f f, y}} M_{y, r e s} \\
& Z_{t h}(L) \approx \frac{L^{2} \Delta T}{4(E I)_{e f f, y}} S_{M_{-} y, t h}
\end{aligned}
$$

The values of the voltage sources and capacitors in the circuit model are:

$$
\begin{aligned}
& V_{\text {res }, \theta}=M_{y, r e s} \quad V_{t h, \theta}=\Delta T S_{M_{-} y, t h} \quad C_{\theta}=\frac{L}{(E I)_{e f f, y}} \\
& V_{\text {res }, z}=\frac{2 M_{y, r e s}}{L} \quad V_{t h, z}=\frac{2 S_{M \_} y, t h}{L} \Delta T \quad C_{z}=\frac{L^{3}}{4(E I)_{e f f, y}}
\end{aligned}
$$

The static deflection of a vertical bimorph with a thermal gradient of slope $m_{t h}$ is found using the same procedure as the lateral multimorph case, following the procedure of equations (10.92)-(10.96):

$$
z_{t h}(L) \approx \frac{L^{2} S_{M_{\_} y, t h} \Delta T}{2(E I)_{e f f, y}}\left[1+\frac{m_{t h} L}{3 \Delta T}\right]
$$

The static mechanical model of a vertical actuator consists of a voltage source representing input force, and a spring (capacitor) that stores potential energy. If the model seeks to determine the tip angle, the capacitance is given by:

$$
C_{\theta}=\frac{1}{k_{\theta}}=\frac{L}{(E I)_{e f f, y}}
$$

and the voltage source has a value of:

$$
V_{t h, \theta}=\frac{L^{2} \Delta T}{2}\left[S_{M_{-} y, t h}+\frac{m_{t h} L}{3 \Delta T}\right]
$$

If the model seeks to determine the tip deflection, the energy methods used in (10.97)-(10.99) may be used. The tip-referred spring constant is: 


$$
k_{z}=\frac{4 E I}{L^{3}}+\frac{4 E I m_{t h}}{3 L^{2}}-\frac{4 E I m_{t h}{ }^{3}}{3\left(L m_{t h}+3\right)^{2}}
$$

The capacitor that represents the tip-referred spring constant is:

$$
C_{z}=\frac{1}{k_{z}}=\frac{L^{3}}{4(E I)_{e f f, y}}\left[\frac{1}{3}+\frac{L m_{t h}+2}{\left(L^{2} m_{t h}^{2}+3 L m_{t h}+3\right)}\right]
$$

The voltage source representing the tip-referred force for a bimorph under thermal gradient loading is:

$$
V_{t h, z}=\frac{q}{C_{z}}=2 S_{M_{-} y, t h} \frac{L^{2} m_{t h}^{2}+3 L m_{t h}+3}{L\left(L m_{t h}+3\right)} \Delta T
$$

In the model below, the displacement at the tip of the actuator is represented by the charge $q$ on the capacitor $C_{z}$. At frequencies above the thermal RC cutoff, the temperature distribution along the length of the actuator becomes important. In order to couple temperature values from multiple points in the thermal model to corresponding points in the mechanical model, one must consider both the tip angle and displacement, since the initial angle of a given segment contributes to the deflection at the end of the segment. The overall deflection is thus weighted by the product of the angle and distance to the tip, as shown in the complete model below. 
Electro-thermo-mechanical Model of Z-actuator

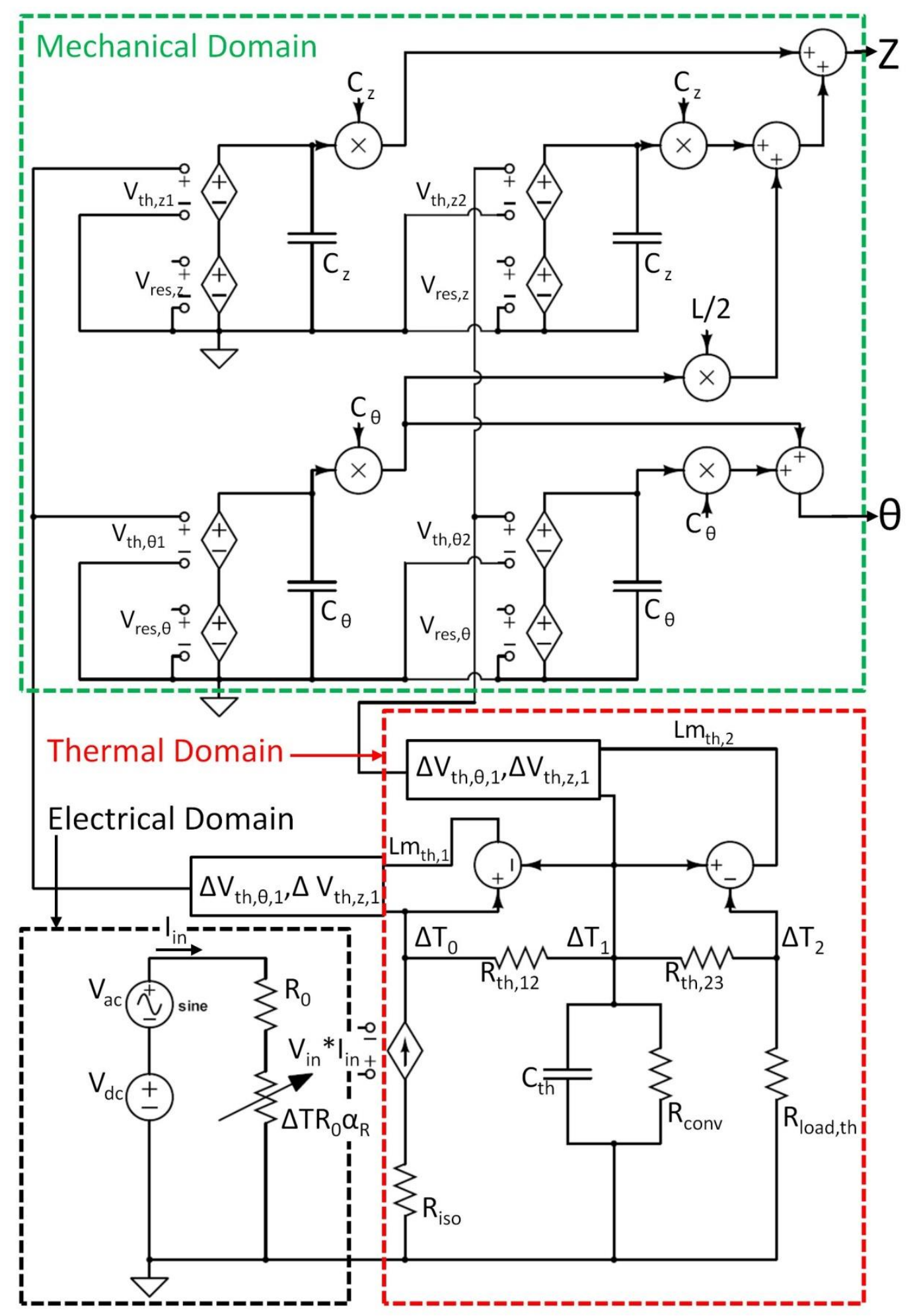




\section{Bibliography}

[1] B. G. E. Moore, “Cramming more components onto integrated circuits," vol. 38, no. 8, 1975.

[2] G. Binnig, H. Rohrer, C. Gerber, and E. Weibel, "Surface studies by scanning tunneling microscopy," Phys. Rev. Lett., vol. 49, no. 1, pp. 57-61, 1982.

[3] F. Ogletree, G. Binnig, H. Rohrer, and I. B. M. Rüshlikon, "25 Years of Scanning Probe Microscopes: How Instrumental Developments Revolutionized Surface Science and Nanotechnology Imaging and Manipulation Facility."

[4] N. Lecture, "Scanning Tunneling Microscopy-from Birth to Adolescence," vol. 26, pp. 606-614, 1987.

[5] D. M. Eigler and E. K. Schweizer, "Positioning single atoms with a scanning tunneling microscope," Nature, vol. 344, pp. 524-526, 1990.

[6] O. Read and W. L. Welch, From tin foil to stereo: evolution of the phonograph. H.W. Sams, 1959.

[7] E. A. Synge, "A suggested method for extending microscopic resolution into the ultra-microscopic region," Philos. Mag. Ser. 7, vol. 6, no. 35, pp. 356-362, 1928.

[8] F. A. Abbott, E.J.; Firestone, "Specifying surface quality: a method based on accurate measurement and comparison," Mech. Eng., vol. 55, pp. 569-572, 1933.

[9] R. Young, "The Topografiner: An Instrument for Measuring Surface Microtopography," Rev. Sci. Instrum., vol. 43, no. 7, p. 999, 1972.

[10] E. A. Ash and G. Nicholls, "Super-resolution Aperture Scanning Microscope," Nature, vol. 237, no. 5357, pp. 510-512, Jun. 1972.

[11] G. Binnig, H. Rohrer, C. Gerber, and E. Weibel, "7× 7 reconstruction on Si (111) resolved in real space," Phys. Rev. Lett., vol. 50, no. 2, 1983.

[12] W. Hofer, A. Foster, and A. Shluger, "Theories of scanning probe microscopes at the atomic scale," Rev. Mod. Phys., vol. 75, no. 4, pp. 1287-1331, Oct. 2003.

[13] J. A. Stroscio and W. J. Kaiser, Scanning Tunneling Microscopy. Elsevier Science, 1993.

[14] G. K. Binnig, "Atomic force microscope and method for imaging surfaces with atomic resolution, US Patent 4724318." Google Patents, 1988.

[15] G. Binnig, C. Quate, and C. Gerber, “Atomic force microscope," Phys. Rev. Lett., 1986.

[16] F. Giessibl and H. Bielefeldt, "Imaging of atomic orbitals with the Atomic Force Microscopeexperiments and simulations," arXiv Prepr. cond-mat/ ..., no. 111, pp. 1-21, 2001. 
[17] F. J. Giessibl, “Advances in atomic force microscopy," Rev. Mod. Phys., vol. 75, no. 3, pp. 949-983, Jul. 2003.

[18] M. a Poggi, E. D. Gadsby, L. a Bottomley, W. P. King, E. Oroudjev, and H. Hansma, "Scanning probe microscopy.," Anal. Chem., vol. 76, no. 12, pp. 3429-43, Jun. 2004.

[19] C. Williams and H. K. Wickramasinghe, "Scanning thermal profiler," Microelectron. Eng., vol. 5, pp. 509-513, 1986.

[20] A. Majumdar, "Scanning Thermal Microscopy," Annu. Rev. Mater. Sci., vol. 29, no. 1, pp. 505-585, Aug. 1999.

[21] R. Pylkki, P. Moyer, and P. West, "Scanning near-field optical microscopy and scanning thermal microscopy," Jpn. J. Appl. Phys., 1994.

[22] Y. B. Gianchandani and K. Najafi, "A silicon micromachined scanning thermal profiler with integrated elements for sensing and actuation," IEEE Trans. Electron Devices, vol. 44, no. 11, pp. 1857-1868, 1997.

[23] S. S. Yang and M. S.-C. Lu, "CMOS integrated cantilevers with sub- $\mu$ m tips for thermal sensing," 2008 3rd IEEE Int. Conf. Nano/Micro Eng. Mol. Syst., pp. 164-169, 2008.

[24] A. Hammiche, "Scanning thermal microscopy: Subsurface imaging, thermal mapping of polymer blends, and localized calorimetry," J. Vac. Sci. Technol. B Microelectron. Nanom. Struct., vol. 14, no. 2, p. 1486, Mar. 1996.

[25] J. Varesi and a. Majumdar, "Scanning Joule expansion microscopy at nanometer scales," Appl. Phys. Lett., vol. 72, no. 1, p. 37, 1998.

[26] W. P. King, T. W. Kenny, K. E. Goodson, G. Cross, M. Despont, U. Dürig, H. Rothuizen, G. K. Binnig, and P. Vettiger, "Atomic force microscope cantilevers for combined thermomechanical data writing and reading," Appl. Phys. Lett., vol. 78, no. 9, p. 1300, 2001.

[27] P. Vettiger, G. Gross, M. Despont, U. Drechsler, U. During, W. Haberle, W. P. King, M. I. Lutwyche, H. Rothuizen, R. Stutz, R. Widmer, and G. K. Binnig, "The 'Millipede'-more than one thousand tips for parallel and dense AFM data storage," 2000 Asia-Pacific Magn. Rec. Conf. Dig. APMRC2000 Mech. Manuf. Asp. HDD (Cat. No.00EX395), pp. MC1/1-MC1/2, 2000.

[28] K. J. Kim, K. Park, J. Lee, Z. M. Zhang, and W. P. King, "Nanotopographical imaging using a heated atomic force microscope cantilever probe," Sensors And Actuators, vol. 136, pp. 95-103, 2007.

[29] B. a. Nelson and W. P. King, "Measuring material softening with nanoscale spatial resolution using heated silicon probes," Rev. Sci. Instrum., vol. 78, no. 2, p. 023702, 2007.

[30] D. E. Steinhauer, C. P. Vlahacos, S. K. Dutta, B. J. Feenstra, F. C. Wellstood, and S. M. Anlage, "Quantitative imaging of sheet resistance with a scanning near-field microwave microscope," Appl. Phys. Lett., vol. 72, no. 7, p. 861, 1998. 
[31] C. Gao and X.-D. Xiang, "Quantitative microwave near-field microscopy of dielectric properties," Rev. Sci. Instrum., vol. 69, no. 11, p. 3846, 1998.

[32] M. Tabib-Azar and D. Su, "0.4 um spatial resolution with $1 \mathrm{GHz}$ (lambda= $30 \mathrm{~cm}$ ) evanescent microwave probe," Rev. Sci. ..., vol. 70, no. 3, pp. 1725-1729, 1999.

[33] K. Lai, W. Kundhikanjana, M. a. Kelly, and Z. X. Shen, "Calibration of shielded microwave probes using bulk dielectrics," Appl. Phys. Lett., vol. 93, no. 12, p. 123105, 2008.

[34] H. P. Huber, I. Humer, M. Hochleitner, M. Fenner, M. Moertelmaier, C. Rankl, a. Imtiaz, T. M. Wallis, H. Tanbakuchi, P. Hinterdorfer, P. Kabos, J. Smoliner, J. J. Kopanski, and F. Kienberger, "Calibrated nanoscale dopant profiling using a scanning microwave microscope," J. Appl. Phys., vol. 111, no. 1, p. 014301, 2012.

[35] H. K. Wickramasinghe, "Progress in scanning probe microscopy," Acta Mater., vol. 48, no. 1, pp. 347-358, Jan. 2000.

[36] T. Ando, N. Kodera, E. Takai, D. Maruyama, K. Saito, and a Toda, "A high-speed atomic force microscope for studying biological macromolecules.," Proc. Natl. Acad. Sci. U. S. A., vol. 98, no. 22, pp. 12468-72, Oct. 2001.

[37] T. R. Albrecht, S. Akamine, T. E. Carver, and C. F. Quate, "Microfabrication of cantilever styli for the atomic force microscope," J. Vac. Sci. Technol. A Vacuum, Surfaces, Film., vol. 8, no. 4, p. 3386, Jul. 1990.

[38] O. Wolter, T. Bayer, and J. Greschner, "Micromachined silicon sensors for scanning force microscopy," J. Vac. Sci. Technol. B Microelectron. Nanom. Struct., vol. 9, no. 2, p. 1353, Mar. 1991.

[39] M. Tortonese, R. C. Barrett, and C. F. Quate, "Atomic resolution with an atomic force microscope using piezoresistive detection," Appl. Phys. Lett., vol. 62, no. 8, p. 834, 1993.

[40] J. A. Harley and T. W. Kenny, "1 = F Noise Considerations for the Design and Process Optimization of Piezoresistive Cantilevers," vol. 9, no. 2, pp. 226-235, 2000.

[41] C. Kocabas and a. Aydinli, "Design and analysis of an integrated optical sensor for scanning force microscopies,” IEEE Sens. J., vol. 5, no. 3, pp. 411-418, Jun. 2005.

[42] N. Blanc, J. Brugger, and N. F. De Rooij, "Scanning force microscopy in the dynamic mode using microfabricated capacitive sensors," vol. 14, no. 2, pp. 901-905, 2007.

[43] B. W. Chui, T. D. Stowe, K. E. Goodson, T. W. Kenny, H. J. Mamin, B. D. Terris, R. P. Ried, and D. Rugar, "Low-stiffness silicon cantilevers with integrated heaters and piezoresistive sensors for high-density AFM thermomechanical data storage," J. Microelectromechanical Syst., vol. 7, no. 1, pp. 69-78, Mar. 1998. 
[44] W. P. King, "Design analysis of heated atomic force microscope cantilevers for nanotopography measurements," J. Micromechanics Microengineering, vol. 15, no. 12, pp. 2441-2448, Dec. 2005.

[45] T. Itoh, C. Lee, and T. Suga, "Deflection detection and feedback actuation using a self-excited piezoelectric $\mathrm{Pb}(\mathrm{Zr}, \mathrm{Ti}) \mathrm{O} 3$ microcantilever for dynamic scanning force microscopy," Appl. Phys. Lett., vol. 69, no. 14, p. 2036, 1996.

[46] E. Algré, Z. Xiong, and M. Faucher, "MEMS ring resonators for laserless AFM with subnanoNewton force resolution," J. Microelectromechanical Syst., pp. 1-13, 2012.

[47] M.-H. Li, J. J. Wu, and Y. B. Gianchandani, "Surface micromachined polyimide scanning thermocouple probes," J. Microelectromechanical Syst., vol. 10, no. 1, pp. 3-9, Mar. 2001.

[48] L. L. Chu, K. Takahata, P. R. Selvaganapathy, Y. B. Gianchandani, and J. L. Shohet, "A micromachined Kelvin probe with integrated actuator for microfluidic and solid-state applications," J. Microelectromechanical Syst., vol. 14, no. 4, pp. 691-698, Aug. 2005.

[49] U. Hartmann, "Magnetic Force Microscopy," Annu. Rev. Mater. Sci., vol. 29, no. 1, pp. 53-87, Aug. 1999.

[50] M. Al-Khafaji, W. Rainforth, M. Gibbs, J. E. L. Bishop, and H. A. Davies, "The effect of tip type and scan height on magnetic domain images obtained by MFM," Magn. IEEE Trans., vol. 32, no. 5, pp. 4138-4140, 1996.

[51] V. V. Talanov, A. Scherz, R. L. Moreland, and A. R. Schwartz, "A near-field scanned microwave probe for spatially localized electrical metrology," Appl. Phys. Lett., vol. 88, no. 13, p. 134106, 2006.

[52] A. N. Reznik and V. V. Talanov, "Quantitative model for near-field scanning microwave microscopy: application to metrology of thin film dielectrics," Rev. Sci. Instrum., vol. 79, no. 11, p. 113708, Nov. 2008.

[53] M. Tabib-Azar, N. S. Shoemaker, and S. Harris, "Non-destructive characterization of materials by evanescent microwaves," Meas. Sci. Technol., vol. 4, no. 5, pp. 583-590, May 1993.

[54] M. Tabib-Azar, D.-P. Su, A. Pohar, S. R. LeClair, and G. Ponchak, "0.4 $\mu \mathrm{m}$ spatial resolution with 1 $\mathrm{GHz}(\lambda=30 \mathrm{~cm})$ evanescent microwave probe," Rev. Sci. Instrum., vol. 70, no. 3, p. 1725, 1999.

[55] K. Lai, M. B. Ji, N. Leindecker, M. a. Kelly, and Z.-X. Shen, "Atomic-force-microscope-compatible near-field scanning microwave microscope with separated excitation and sensing probes.," Rev. Sci. Instrum., vol. 78, no. 6, p. 063702, Jun. 2007.

[56] Y. Yang, K. Lai, Q. Tang, W. Kundhikanjana, M. a Kelly, K. Zhang, Z. Shen, and X. Li, "Batchfabricated cantilever probes with electrical shielding for nanoscale dielectric and conductivity imaging," J. Micromechanics Microengineering, vol. 22, no. 11, p. 115040, Nov. 2012. 
[57] M. Lutwyche, C. Andreoli, G. Binnig, J. Brugger, U. Drechsler, W. Haeberle, H. Rohrer, H. Rothuizen, and P. Vettiger, "Microfabrication and parallel operation of 5×5 2D AFM cantilever arrays for data storage and imaging," Proc. MEMS 98. IEEE. Elev. Annu. Int. Work. Micro Electro Mech. Syst. An Investig. Micro Struct. Sensors, Actuators, Mach. Syst. (Cat. No.98CH36176), pp. 8-11, 1998.

[58] P. Vettiger, G. Cross, M. Despont, U. Drechsler, U. Durig, B. Gotsmann, W. Haberle, M. a. Lantz, H. E. Rothuizen, R. Stutz, and G. K. Binnig, "The 'millipede' - nanotechnology entering data storage," IEEE Trans. Nanotechnol., vol. 1, no. 1, pp. 39-55, Mar. 2002.

[59] Y. Kim, "PZT cantilever array integrated with piezoresistor sensor for high speed parallel operation of AFM," Sensors Actuators A Phys., vol. 103, no. 1-2, pp. 122-129, Jan. 2003.

[60] D. Bullen and C. Liu, "Electrostatically actuated dip pen nanolithography probe arrays," Sensors Actuators A Phys., vol. 125, no. 2, pp. 504-511, Jan. 2006.

[61] S. McNamara, A. S. Basu, J. Lee, and Y. B. Gianchandani, "Ultracompliant thermal probe array for scanning non-planar surfaces without force feedback," J. Micromechanics Microengineering, vol. 15, no. 1, pp. 237-243, Jan. 2005.

[62] D. Barrettino, S. Hafizovic, T. Volden, J. Sedivy, K. Kirstein, a. Hierlemann, and H. Baltes, "CMOS monolithic atomic force microscope," 2004 Symp. VLSI Circuits. Dig. Tech. Pap. (IEEE Cat. No.04CH37525), pp. 306-309, 2004.

[63] S. Hafizovic, D. Barrettino, T. Volden, J. Sedivy, K.-U. Kirstein, O. Brand, and A. Hierlemann, "Single-chip mechatronic microsystem for surface imaging and force response studies.," Proc. Natl. Acad. Sci. U. S. A., vol. 101, no. 49, pp. 17011-5, Dec. 2004.

[64] S. Akamine, T. R. Albrecht, M. J. Zdeblick, and C. F. Quate, "Microfabricated scanning tunneling microscope," IEEE Electron Device Lett., vol. 10, no. 11, pp. 490-492, 1989.

[65] Y. Xu, N. MacDonald, and S. Miller, "Integrated micro-[?scanning tunneling microscope," Appl. Phys. Lett., vol. 67, no. October, pp. 2305-2307, 1995.

[66] D. Jingyan and P. Ferreira, "Electrostatically Actuated Cantilever With SOI-MEMS Parallel Kinematic XY Stage," J. Microelectromechanical Syst., vol. 18, no. 3, pp. 641-651, 2009.

[67] K. Amponsah and A. Lal, "MULTIPLE TIP NANO P PROBE ACTUATORS WITH INTE GRATED JFETS Kwame Amponsah and Amit Lal," no. February, pp. 1356-1359, 2012.

[68] J. Liu, L. Draghi, J. a. Bain, T. E. Schlesinger, and G. K. Fedder, "Three-DOF CMOS-MEMS probes with embedded piezoresistive sensors," 2010 IEEE 23rd Int. Conf. Micro Electro Mech. Syst., pp. 284-287, Jan. 2010.

[69] S. M. Sze, VLSI technology. McGraw-Hill, 1983.

[70] R. C. Jäger, Introduction to Microelectronic Fabrication. Prentice Hall PTR, 2002. 
[71] M. Madou, Fundamentals of Microfabrication: The Science of Miniturization. Taylor \& Francis Limited, 2011.

[72] G. T. A. Kovacs, Micromachined Transducers Sourcebook. New York: WCB/McGraw-Hill, 1998.

[73] J. M. Bustillo, R. T. Howe, and R. S. Muller, "Surface micromachining for microelectromechanical systems," Proc. IEEE, vol. 86, no. 8, pp. 1552-1574, 1998.

[74] C. Hagleitner, a Hierlemann, D. Lange, a Kummer, N. Kerness, O. Brand, and H. Baltes, "Smart single-chip gas sensor microsystem.," Nature, vol. 414, no. 6861, pp. 293-6, Nov. 2001.

[75] J. Giner, a Uranga, J. L. Muñóz-Gamarra, E. Marigó, and N. Barniol, “A fully integrated programmable dual-band RF filter based on electrically and mechanically coupled CMOS-MEMS resonators," J. Micromechanics Microengineering, vol. 22, no. 5, p. 055020, May 2012.

[76] G. K. Fedder, R. T. Howe, and E. P. Quevy, "Technologies for Cofabricating MEMS and Electronics," Proc. IEEE, vol. 96, no. 2, pp. 306-322, Feb. 2008.

[77] H. Baltes, O. Brand, G. K. Fedder, C. Hierold, J. G. Korvink, and O. Tabata, CMOS-MEMS: Advanced Micro and Nanosystems. Wiley, 2008.

[78] R. R. Mansour, "RF MEMS-CMOS Device Integration," IEEE Microw. Mag., no. February 2012, pp. 39-56, 2013.

[79] W. et. el. Frobenius, "A microminiature solid-state capacitive blood pressure transducer with improved sensitivity," IEEE Trans. Biomed. Eng., pp. 1-10, 1973.

[80] K. Najafi and K. Wise, "An implantable multielectrode array with on-chip signal processing," SolidState Circuits, IEEE J., no. December, 1986.

[81] K. D. Wise, "WIRELESS IMPLANTABLE MICROSYSTEMS : Creating a Revolution in Health Care," pp. 26-29, 2012.

[82] F. Laermer and A. Schilp, "Method of anisotropically etching silicon," US Patent 6284148. Google Patents, p. 7, 1996.

[83] M. Offenberg and F. Larmer, "Novel process for a monolithic integrated accelerometer," SolidState Sensors Actuators, TRANSDUCERS '95, pp. 589-592, 1995.

[84] T. J. Brosnihan, J. M. Bustillo, A. P. Pisano, and R. T. Howe, "Embedded Interconnect and Electrical Isolation for High-Aspect-Ratio, \{SOI\} Inertial Instruments," in Transducers '97, pp. 637640.

[85] S. Lewis, S. Alie, T. Brosnihan, C. Core, T. Core, R. Howe, J. Geen, D. Hollocher, M. Judy, J. Memishian, K. Nunan, R. Paine, S. Sherman, B. Tsang, and B. Wachtmann, Integrated sensor and electronics processing for $>10^{\wedge} 8$ "iMEMS" inertial measurement unit components, vol. 00, no. c. leee, 2003, pp. 39.1.1-39.1.4. 
[86] J. H. Smith, S. Montague, J. J. Sniegowski, J. R. Murray, and P. J. McWhorter, Embedded micromechanical devices for the monolithic integration of MEMS with CMOS, no. 100. leee, 1995, pp. 609-612.

[87] R. N. Candler, S. Member, W. Park, H. Li, G. Yama, A. Partridge, M. Lutz, and T. W. Kenny, "Single Wafer Encapsulation of MEMS Devices," IEEE Trans. Adv. Packag., vol. 26, no. 3, pp. 227-232, 2003.

[88] R. N. Candler, M. A. Hopcroft, B. K. B. Kim, W.-T. P. W.-T. Park, R. Melamud, M. Agarwal, G. Yama, A. Partridge, M. Lutz, and T. W. Kenny, Long-Term and Accelerated Life Testing of a Novel SingleWafer Vacuum Encapsulation for MEMS Resonators, vol. 15, no. 6. IEEE-INST ELECTRICAL ELECTRONICS ENGINEERS INC, 2006, pp. 1446-1456.

[89] K. Harada, K. Ikeda, H. Kuwayama, and H. Murayama, "Various applications of resonant pressure sensor chip based on 3-D micromachining," Sensors Actuators A Phys., vol. 73, no. 3, pp. 261266, 1999.

[90] E. Eleftheriou, T. Antonakopoulos, G. K. Binnig, G. Cherubini, M. Despont, a. Dholakia, U. Durig, M. a. Lantz, H. Pozidis, H. E. Rothuizen, and P. Vettiger, "Millipede-a MEMS-based scanning-probe data-storage system," IEEE Transactions on Magnetics, vol. 39, no. 2. pp. 938-945, Mar-2003.

[91] L. J. Hornbeck, Digital Light Processing and MEMS: an overview. 1996, pp. 7-8.

[92] H. Takeuchi, E. Quevy, S. A. Bhave, T.-J. K. T.-J. King, and R. T. Howe, Ge-blade damascene process for post-CMOS integration of nano-mechanical resonators, vol. 25, no. 8. 2004, pp. 529-531.

[93] H. Takeuchi, A. Wung, X. S. X. Sun, R. T. Howe, and T.-J. K. T.-J. King, Thermal budget limits of quarter-micrometer foundry CMOS for post-processing MEMS devices, vol. 52, no. 9. 2005, pp. 2081-2086.

[94] S. Sedky, A. Witvrouw, H. Bender, and K. Baert, Experimental determination of the maximum post-process annealing temperature for standard CMOS wafers, vol. 48, no. 2. 2001, pp. 377-385.

[95] J. F. Carpentier, A. Cathelin, C. Tilhac, P. Garcia, P. Persechini, P. Conti, P. Ancey, G. Bouche, G. Caruyer, D. Belot, C. Arnaud, C. Billard, G. Parat, J. B. David, P. Vincent, M. A. Dubois, and C. Enz, A SiGe:C BiCMOS WCDMA zero-IF RF front-end using an above-IC BAW filter, no. 1. 2005.

[96] M. Aissi, E. Tournier, M. A. Dubois, G. Parat, and R. Plana, A 5.4GHz 0.35/sp/ mu/m BiCMOS FBAR Resonator Oscillator in Above-IC Technology. 2006, pp. 1228-1235.

[97] D. L. Polla, R. S. Muller, and R. M. White, Integrated multisensor chip, vol. 7, no. 4. 1986, pp. 254256.

[98] K. A. Honer and G. T. A. Kovacs, "Integration of sputtered silicon microstructures with prefabricated CMOS circuitry," Sensors Actuators A Phys., vol. 91, no. 3, pp. 386-397, 2001. 
[99] P. Alpuim, V. Chu, and J. P. Conde, "Electronic and structural properties of doped amorphous and nanocrystalline silicon deposited at low substrate temperatures by radio-frequency plasmaenhanced chemical vapor deposition," J. Vac. Sci. Technol. A Vacuum, Surfaces, Film., vol. 21, no. 4, p. 1048, 2003.

[100] J. M. Bustillo, G. K. Fedder, C. T. C. Nguyen, and R. T. Howe, "Process technology for the modular integration of CMOS and polysilicon microstructures," Microsyst. Technol., vol. 1, no. 1, pp. 3041, 1994.

[101] A. E. Franke, J. M. Heck, T.-J. K. T.-J. King, and R. T. Howe, Polycrystalline silicon-germanium films for integrated microsystems, vol. 12, no. 2. 2003, pp. 160-171.

[102] S. Sugiyama and K. E. N. Kawahata, "Tactile Image Detection Using a Ik-element Silicon Pressure Sensor Array fabncated for the detection of a high-resolution with functions for signal amphficatlon and offset," Sensors Actuators A Phys., vol. 23, pp. 397-400, 1990.

[103] P. Kruse, R. Dodson, S. Anderson, L. Kantor, M. Knipfer, T. Mcmanus, A. Wood, and T. Rezachek, "Infrared Imager Employing 160x120 Pixel Uncooled Bolometer Array," Proc. SPIE, vol. 3436, no. July 1998, pp. 572-577, 2001.

[104] J. D. Zahn, K. J. Gabriel, and G. K. Fedder, "A direct plasma etch approach to high aspect ratio polymer micromachining with applications in bioMEMS and CMOS-MEMS," Tech. Dig. MEMS 2002 IEEE Int. Conf. Fifteenth IEEE Int. Conf. Micro Electro Mech. Syst. (Cat. No.02CH37266), pp. 137-140.

[105] I. T. Rueckes and B. M. Segal, United States Patent NANOTUBE FILMS AND ARTICLES, vol. 111111, no. 12.2004 , p. 39.

[106] M. Parameswaran, H. P. Baltes, and A. M. Robinson, "Polysilicon microbridge fabrication using standard CMOS technology," in Technical Digest IEEE Solid-State Sensor and Actuator Workshop, 1988.

[107] J. S. Suehle, R. E. Cavicchi, M. Gaitan, and S. Semancik, Tin oxide gas sensor fabricated using CMOS micro-hotplates and in-situ processing, vol. 14, no. 3. 1993, pp. 118-120.

[108] Z. Olgun, O. Akar, H. Kulah, and T. Akin, An integrated thermopile structure with high responsivity using any standard CMOS process, vol. 2. 1997, pp. 1263-1266.

[109] G. K. Fedder, S. Santhanam, M. L. Reed, S. C. Eagle, D. F. Guillou, M. S. C. Lu, and L. R. Carley, Laminated high-aspect-ratio microstructures in a conventional CMOS process, vol. 57, no. 2. Elsevier, 1996, pp. 103-110.

[110] D. Westberg, O. Paul, G. I. Andersson, and H. Baltes, "Surface micromachining by sacrificial aluminium etching," J. Micromechanics Microengineering, vol. 6, no. 4, pp. 376-384, Dec. 1996.

[111] A. Jain and H. Xie, "A single-crystal silicon micromirror for large bi-directional 2D scanning applications," Sensors Actuators A Phys., vol. 130-131, pp. 454-460, 2006. 
[112] J. (Jack) Luo, J. H. He, A. Flewitt, D. F. Moore, S. M. Spearing, N. a. Fleck, and W. I. Milne, "Development of all metal electrothermal actuator and its applications," J. Microlithogr. Microfabr. Microsystems, vol. 4, no. 2, p. 023012, 2005.

[113] Y. B. Gianchandani and K. Najafi, "A silicon micromachined scanning thermal profiler with integrated elements for sensing and actuation," IEEE Trans. Electron Devices, vol. 44, no. 11, pp. 1857-1868, 1997.

[114] H. Fujita, "Microactuators and Micromachines," Proc. IEEE, vol. Vol. 86, N, pp. 1721-1732, 1998.

[115] A. Nathan and H. Baltes, Microtransducer CAD, Physical and Computational Aspects. Vienna: Vienna: Springer-Verlag, 1999.

[116] L. Que, J. S. Park, and Y. B. Gianchandani, "Bent-Beam Electro-Thermal Actuators for High Force Applications," in \{MEMS\} '99, International Conference on Micro Electro Mechanical Systems, 1999, pp. 31-36.

[117] L. Que, J.-S. Park, and Y. B. Gianchandani, "Bent-Beam Electrothermal Actuators - Part I: Single Beam and Cascaded Devices," J. Microelectromechanical Syst., vol. Vol. 10, N, pp. 247-254, 2001.

[118] A. Tuantranont, V. M. Bright, L.-A. Liew, W. Zhang, and Y. C. Lee, "Smart Phase-Only Micromirror Array Fabricated by Standard \{CMOS\} Process," in Proceedings of The13th Annual International Conference on Micro Electromechanical Systems \{(MEMS\} 2000), 2000, pp. 455-460.

[119] W. C. Chen, C. C. Chu, J. Hsieh, and W. Fang, "A Reliable Single-Layer Out-of-Plane Micromachined Thermal Actuator," Sensors Actuators A, vol. 103, pp. 48-58, 2003.

[120] J. Ji, J. Chaney, M. Kaviany, P. L. Bergstrom, and K. D. Wise, "Microactuation Based on ThermallyDriven Phase-Change," in Solid-State Sensors and Actuators, TRANSDUCERS '91, 1991, pp. 10371040.

[121] E. Carlen and C. Mastrangelo, "Electrothermally Activated Paraffin Microactuators," J. Microelectromechanical Syst., vol. Vol. 11, N, pp. 165-174, 2002.

[122] D. Johnson, "Vacuum-Deposited \{TiNi\} Shape Memory Film: Characterization and Applications in Microdevices," J. Micromech. Microeng., vol. 1, pp. 34-41, 1991.

[123] P. Krulevitch, A. P. Lee, P. B. Philip, B. Ramsey, J. C. Trevino, J. Hamilton, and M. A. Northrup, "Thin Film Shape Memory Alloy Microactuators," J. Microelectromechanical Syst., vol. Vol. 5, No, pp. 270-281, 1996.

[124] T. Ikehara, M. Tanaki, S. Shimada, and H. Matsuda, "Optically-Driven Actuator Using PhotoInduced Phase-Transition Material," in \{MEMS\} 2001, International Conference on Micro Electro Mechanical Systems, 2001, pp. 256-259. 
[125] A. Henning, J. Fitch, D. Hopkins, L. Lilly, R. Faeth, E. Falsken, and M. Zdeblick, "A Thermopneumatically Actuated Microvalve for Liquid Expansion and Proportional Control," in Solid-State Sensors and Actuators, \{TRANSDUCERS\} '97, 1997, pp. 825-828.

[126] A. Oliver, S. Vigil, and Y. Gianchandani, "Photothermal Surface-Micromachined Actuators," IEEE Trans. Electron Devices, vol. Vol. 50, N, pp. 1156-1157, 2003.

[127] T. Akiyama and K. Shono, "Controlled Stepwise Motion in Polysilicon Microstructures," J. Microelectromechanical Syst., vol. Vol. 2, No, pp. 106-110, 1993.

[128] R. Linderman and V. M. Bright, "Nanometer Precision Positioning Robots Utilizing Optimized Scratch Drive Actuators," Sensors Actuators A, vol. 91, pp. 292-300, 2001.

[129] W. C. Tang, T.-C. H. Nguyen, and R. T. Howe, "Laterally Driven Polysilicon Resonant Microstructures," in An Investigation of Micro Structures, Sensors, Actuators, Machines and Robots, 1989, pp. 53-59.

[130] W. Ye, S. Mukherjee, and N. MacDonald, "Optimal Shape Design of an Electrostatic Comb Drive in Microelectromechanical Systems," J. Microelectromechanical Syst., vol. Vol. 7, No, pp. 16-26, 1998.

[131] Y. Nemirovsky and O. Bochobza-Degani, "A Methodology and Model for the Pull-In Parameters of Electrostatic Actuators," J. Microelectromechanical Syst., vol. Vol. 10, N, pp. 601-615, 2001.

[132] R. Nadal-Guardia, A. Dehe, R. Aigner, and L. Castaner, "Current Drive Methods to Extend the Range of Travel of Electrostatic Microactuator Beyond the Voltage Pull-in-Point," J. Microelectromechanical Syst., vol. Vol. 11, N, pp. 255-263, 2002.

[133] M. Hisanaga, T. Koumura, and T. Hattori, "Fabrication of 3-Dimensionally Shaped Si Diaphragm Dynamic Focusing Mirror," in MEMS1993, International Conference on Micro Electro Mechanical Systems, 1993, pp. 30-35.

[134] C. Goldsmith, T.-H. L. amd B. Powers, W.-R. Wu, and B. Norvell, "Micromechanical Membrane Switches for Microwave Applications," in \{MTT\}-S International, 1995, vol. Vol. 1, pp. 91-94.

[135] M. Shikida, K. Sato, and T. Harada, "Fabrication of a S-Shaped Microactuator," J. Microelectromechanical Syst., vol. Vol. 6, No, pp. 18-24, 1997.

[136] J. D. Jacobson, S. H. Goodwin-Johansson, S. M. Bobbio, C. A. Bartlett, and L. Y. Yadon, "Integrated Force Arrays: Theory and Modeling of Static Operation," J. Microelectromechanical Syst., vol. Vol. 4, No, pp. 139-150, 1995.

[137] M. Yamaguchi, S. Kawamura, K. Minami, and M. Esashi, "Control of Distributed Electrostatic Microstructures," J. Micromech. Microeng., vol. 3, pp. 90-95, 1993.

[138] P. Van Kessel, L. Hornbeck, R. Meier, and M. Douglass, "A MEMS-Based Projection display," Proc. IEEE, vol. Vol. 86, N, pp. 1687-1704, 1998. 
[139] T. Higuchi, Y. Yamagata, K. Furutani, and K. Kudoh, "Precise Positioning Mechanism Utilizing Rapid Deformations of Piezoelectric Elements," in \{MEMS\} 1990, \{IEEE\} Micro Electro Mechanical Syst. Workshop, 1990, pp. 222-226.

[140] A. micro mobile mechanism using thermal expansion and its theoretical analysis, "A Micro Mobile Mechanism Using Thermal Expansion and its Theoretical Analysis," in \{MEMS\} 1994, \{IEEE\} Micro Electro Mechanical Syst. Workshop, 1994, pp. 142-147.

[141] O. Ohmichi, Y. Yamagata, and T. Higuchi, "Micro Impact Drive Mechanisms Using Optically Excited Thermal Expansion," J. Microelectromechanical Syst., vol. Vol. 6, No, pp. 200-207, 1997.

[142] D. Niarchos, "Magnetic \{MEMS\}: Key Issues and some Applications," Sensors Actuators A, vol. 106, pp. 255-262, 2003.

[143] J. Judy, R. Muller, and H. Zappe, "Magnetic Microactuation of Polysilicon Flexure Structures," J. Microelectromechanical Syst., vol. Vol. 4, No, pp. 162-169, 1995.

[144] N. Miki and I. Shimoyama, "Dynamics of a Microflight Mechanism With Magnetic Rotational Wings in an Alternating Magnetic Field," J. Microelectromechanical Syst., vol. Vol. 11, N, pp. 584591, 2002.

[145] L. Lagorce, O. Brand, and M. Allen, "Magnetic Microactuators Based on Polymer Magnets," J. Microelectromechanical Syst., vol. Vol. 8, No, pp. 2-9, 1999.

[146] T. Bourouina, E. Lebrasseur, G. Reyne, A. Debray, H. Fujita, A. Ludwig, E. Quandt, H. Muro, T. Oki, and A. Asaoka, "Integration of Two Degree-of-Freedom Magnetostrictive Actuation and Piezoresistive Detection: Application of a Two-Dimensional Optical Scanner," J. Microelectromechanical Syst., vol. Vol. 11, N, pp. 355-361, 2002.

[147] H. Savage, A. Clark, and M. Spano, "Strain-Field Relationships in Rare-Earth Iron Alloys," IEEE Trans. Magn., vol. Vol. 20, N, pp. 1449-1450, 1984.

[148] N. J. Grabhem, N. M. White, and S. P. Beeby, "Thick-Film Magnetostrictive Material for $\{$ MEMS\}," Electron. Lett., vol. Vol. 36, N, pp. 332-334, 2000.

[149] S. D. Senturia, Microsystem Design. Springer, 2000.

[150] D. Lange, O. Brand, and H. Baltes, CMOS Cantilever Sensor Systems: Atomic-Force Microscopy and Gas Sensing Applications. Springer, 2002.

[151] A. Hajjam, J. C. Wilson, A. Rahafrooz, and S. Pourkamali, "Self-sustained micromechanical resonant particulate microbalance/counters," 2011 IEEE 24th Int. Conf. Micro Electro Mech. Syst., pp. 629-632, Jan. 2011.

[152] L. Yin and G. K. Ananthasuresh, "Design of Distributed Compliant Mechanisms," Mech. Based Des. Struct. Mach., vol. 31, no. 2, pp. 151-179, Jan. 2003. 
[153] G. K. Ananthasuresh, Optimal Synthesis Methods for Mems. Kluwer Academic Publishers, 2003.

[154] A. Geisberger, A. Jungen, N. Sarkar, M. Ellis, and G. Skidmore, "Modeling Electrothermal Plastic Deformation Self-Assembly," in Technical Proceedings of the 2003 Nanotechnology Conference and Trade Show, Vol.1, 2003, pp. 482-485.

[155] N. Lobontiu and E. Garcia, Mechanics of Microelectromechanical Systems. Springer, 2005.

[156] J. Tamayo, "Study of the noise of micromechanical oscillators under quality factor enhancement via driving force control," J. Appl. Phys., vol. 97, no. 4, p. 044903, 2005.

[157] A. Rahafrooz and S. Pourkamali, "High-frequency thermally actuated electromechanical resonators with piezoresistive readout," Electron Devices, IEEE Trans., vol. 58, no. 4, pp. 12051214, 2011.

[158] R. J. Cameron, C. M. Kudsia, and R. R. Mansour, Microwave filters for communication systems: fundamentals, design, and applications. Wiley-Interscience, 2007.

[159] P. French and A. Evans, "Piezoresistance in polysilicon," Electron. Lett., vol. 20, no. 24, pp. 9991000, 1984.

[160] P. French and A. Evans, "Piezoresistance in polysilicon and its applications to strain gauges," Solid. State. Electron., vol. 32, no. 1, 1989.

[161] V. A. Gridchin, V. M. Lubimsky, and M. P. Sarina, "Piezoresistive Properties of Polysilicon Films," Sensors Actuators A, vol. 49, pp. 67-72, 1995.

[162] a A. Barlian, W.-T. Park, J. R. Mallon, A. J. Rastegar, and B. L. Pruitt, "Review: Semiconductor Piezoresistance for Microsystems.," Proc. IEEE. Inst. Electr. Electron. Eng., vol. 97, no. 3, pp. 513552, Jan. 2009.

[163] R. M. Panas, M. a. Cullinan, and M. L. Culpepper, "Design of piezoresistive-based MEMS sensor systems for precision microsystems," Precis. Eng., vol. 36, no. 1, pp. 44-54, Jan. 2012.

[164] J. Harkey and T. Kenny, "1/f noise considerations for the design and process optimization of piezoresistive cantilevers," ... Syst. J., vol. 9, no. 2, pp. 226-235, 2000.

[165] J. Liu, M. Noman, J. a. Bain, T. E. Schlesinger, and G. K. Fedder, "Polysilicon sensors for CMOSMEMS electrothermal probes," TRANSDUCERS 2009 - 2009 Int. Solid-State Sensors, Actuators Microsystems Conf., pp. 2425-2428, Jun. 2009.

[166] C. T.-C. Nguyen and R. T. Howe, "An integrated CMOS micromechanical resonator high-Q oscillator," IEEE J. Solid-State Circuits, vol. 34, no. 4, pp. 440-455, Apr. 1999.

[167] A. Rahafrooz and S. Pourkamali, "Thermal-Piezoresistive Energy Pumps in Micromechanical Resonant Structures," IEEE Trans. Electron Devices, vol. 59, no. 12, pp. 3587-3593, Dec. 2012. 
[168] A. Rahafrooz and S. Pourkamali, "ZERO BIAS OPERATION OF THERMAL-PIEZORESISTIVE MICROMECHANICAL RESONATORS VIA INTERNAL ELECTROMECHANICAL MIXING a ) b )," pp. 375-378, 2012.

[169] H. Hölscher, D. Ebeling, and U. D. Schwarz, "Theory of Q-Controlled dynamic force microscopy in air," J. Appl. Phys., vol. 99, no. 8, p. 084311, 2006.

[170] D. Rugar and P. Grutter, "Mechanical parametric amplification and thermomechanical noise squeezing," Phys. Rev. Lett., vol. 67, no. 6, 1991.

[171] K. Turner, S. Miller, and P. Hartwell, "Five parametric resonances in a microelectromechanical system," Nature, vol. 396, no. November, pp. 149-152, 1998.

[172] J. F. Rhoads, S. W. Shaw, and K. L. Turner, "The nonlinear response of resonant microbeam systems with purely-parametric electrostatic actuation," J. Micromechanics Microengineering, vol. 16, no. 5, pp. 890-899, May 2006.

[173] R. Karabalin, X. Feng, and M. Roukes, "Parametric nanomechanical amplification at very high frequency," Nano Lett., 2009.

[174] A. Geisberger, N. Sarkar, M. Ellis, and G. Skidmore, "Electrothermal Properties and Modeling of Polysilicon Microthermal Actuators," J. Microelectromechanical Syst., vol. Vol. 12, N, pp. 513523, 2003.

[175] C. Van Vroonhoven, M. Kashmiri, and K. Makinwa, "CMOS Temperature Sensors Based on Thermal Diffusion," no. October, pp. 7-10, 2009.

[176] D. Platz, D. Forchheimer, E. a Tholén, and D. B. Haviland, "Interaction imaging with amplitudedependence force spectroscopy.," Nat. Commun., vol. 4, p. 1360, Jan. 2013.

[177] H. Toshiyoshi and D. Kobayashi, "Microelectromechanical digital-to-analog converters of displacement for step motion actuators," Microelectromechanical Syst. Syst. J., vol. 9, no. 2, pp. 218-225, 2000.

[178] R. Yeh, R. Conant, and K. Pister, "Mechanical digital-to-analog converters," Dig. Tech. Pap. 10th Int. Solid-State Sensors, Actuators Microsystems Conf., 1999.

[179] S. Jang, "A model of 1/f noise in polysilicon resistors," Solid. State. Electron., vol. 33, no. 9, 1990.

[180] J. Y. W. Seto, "The electrical properties of polycrystalline silicon films," J. Appl. Phys., vol. 46, no. 12 , p. 5247, 1975.

[181] N. Lu and L. Gerzberg, "Modeling and optimization of monolithic polycrystalline silicon resistors," Electron Devices, IEEE Trans., no. I, pp. 818-830, 1981.

[182] J. C. Doll, S.-J. Park, and B. L. Pruitt, "Design optimization of piezoresistive cantilevers for force sensing in air and water.," J. Appl. Phys., vol. 106, no. 6, p. 64310, Sep. 2009. 
[183] A. S. Basu and Y. B. Gianchandani, "Nanopatterning: Surfaces feel the heat.," Nat. Nanotechnol., vol. 4, no. 10, pp. 622-3, Oct. 2009.

[184] N. Sarkar, M. Azizi, S. Fouladi, and R. R. Mansour, "Self-actuating scanning microwave microscopy probes," 2012 IEEE/MTT-S Int. Microw. Symp. Dig., pp. 1-3, Jun. 2012.

[185] N. Sarkar and R. Mansour, "A CMOS-MEMS scanning probe microscope with integrated position sensors," 2008 1st Microsystems Nanoelectron. Res. Conf., pp. 77-80, Oct. 2008.

[186] N. Sarkar and R. Mansour, "CMOS-MEMS atomic force microscope," Transducers 2011, pp. 26102613, Jun. 2011.

[187] N. Sarkar, R. R. Mansour, and K. Trainor, "Forced Oscillation and Higher Harmonic Detection in an Integrated CMOS-MEMS Scanning Probe Microscope," in 2012 Hilton Head Solid-State Sensors, Actuators, and Microsystems Workshop, 2012, no. c.

[188] N. Sarkar, G. Lee, and R. Mansour, "CMOS-MEMS dynamic FM atomic force microscope," SolidState Sensors, Actuators ..., 2013.

[189] S. Schmucker, N. Kumar, and J. Abelson, "Field-directed sputter sharpening for tailored probe materials and atomic-scale lithography," Nat. Commun., 2012.

[190] P. J. Gilgunn, "SOI-CMOS-MEMS Electrothermal Micromirror Arrays," PhD Diss., no. April, 2010.

[191] S. Park and C. Quate, "Theories of the feedback and vibration isolation systems for the scanning tunneling microscope," Rev. Sci. Instrum., 1987.

[192] C. W. De Silva, "de Silva, Clarence W. 'Vibration Design and Control'," 2000.

[193] M. J. Martin, B. H. Houston, J. W. Baldwin, and M. K. Zalalutdinov, "Damping Models for Microcantilevers, Bridges, and Torsional Resonators in the Free-Molecular-Flow Regime," J. Microelectromechanical Syst., vol. 17, no. 2, pp. 503-511, Apr. 2008.

[194] J. W. Nilsson and S. Riedel, Electric Circuits. Prentice Hall, 2010.

[195] S. T. Todd, "An Electrothermomechanical Lumped Element Model of an Electrothermal Bimorph Actuator," J. Microelectromechanical Syst., vol. 17, no. 1, pp. 213-225, Feb. 2008.

[196] J. B. J. Fourier, Théorie analytique de la chaleur. 1822.

[197] K. D. Cole, Heat Conduction Using Green's Functions. Taylor \& Francis Group, 2010.

[198] R. B. Guenther and J. W. Lee, Partial Differential Equations of Mathematical Physics and Integrated Equations. Dover Publications, Incorporated, 1988.

[199] L. Debnath and T. Myint-U, Linear partial differential equations for scientists and engineers. Birkhauser, 2007. 
[200] A. A. Geisberger and N. Sarkar, "Techniques in mems microthermal actuators and their applications," Micro, pp. 1-52.

[201] O. Ozsun, B. E. Alaca, A. D. Yalcinkaya, M. Yilmaz, M. Zervas, and Y. Leblebici, "On heat transfer at microscale with implications for microactuator design," Control, vol. 19, 2009.

[202] M. Mayyas, P. S. Shiakolas, W. H. Lee, D. Popa, and H. Stephanou, "Static and dynamic modeling of thermal microgripper," 2006 14th Mediterr. Conf. Control Autom., no. 817, pp. 1-6, Jun. 2006.

[203] B. Borovic, F. L. Lewis, D. Agonafer, E. S. Kolesar, M. M. Hossain, and D. O. Popa, "Method for Determining a Dynamical State\&\#8211;Space Model for Control of Thermal MEMS Devices," J. Microelectromechanical Syst., vol. 14, no. 5, pp. 961-970, Oct. 2005.

[204] T. Bechtold, E. B. Rudnyi, and J. G. Korvink, "Dynamic electro-thermal simulation of microsystems-a review," J. Micromechanics Microengineering, vol. 15, no. 11, pp. R17-R31, Nov. 2005.

[205] V. Székely, "On the representation of infinite-length distributed RC one-ports," Circuits Syst. IEEE Trans., vol. 38, no. 7, pp. 711-719, 1991.

[206] J. Lienemann, D. Billger, E. B. Rudnyi, A. Greiner, and J. G. Korvink, "MEMS compact modeling meets model order reduction: Examples of the application of Arnoldi methods to microsystem devices," Nsti Nanotech 2004 Vol 2 Tech. Proc., pp. 303-306, 2004.

[207] R. Freund, "Krylov-subspace methods for reduced-order modeling in circuit simulation," J. Comput. Appl. Math., vol. 123, no. 1-2, pp. 395-421, 2000.

[208] E. B. Rudnyi and J. G. Korvink, "Model order reduction for large scale finite element engineering models," ECCOMAS CFD Netherlands, 2006.

[209] D. Yan, A. Khajepour, and R. Mansour, "Modeling of Two-Hot-Arm Horizontal Thermal Actuator," J. Micromech. Microeng., vol. Vol. 13, pp. 312-322, 2003.

[210] D. Yan, A. Khajepour, and R. Mansour, "Design and Modeling of a \{MEMS\} Bidirectional Vertical Thermal Actuator," J. Micromech. Microeng., vol. Vol. 14, pp. 841-850, 2004.

[211] S. Pal, "Modeling and reliability of electrothermal micromirrors," PhD Thesis, Univ. Florida, 2011.

[212] O. Ozsun, B. E. Alaca, A. D. Yalcinkaya, M. Yilmaz, M. Zervas, and Y. Leblebici, "On heat transfer at microscale with implications for microactuator design," J. Micromechanics Microengineering, vol. 19, no. 4, p. 045020, Apr. 2009.

[213] J. Pelesko and D. Bernstein, Modeling Mems and Nems. 2003.

[214] Y. B. Gianchandani and K. Najafi, "Bent-Beam Strain Sensors," J. Microelectromechanical Syst., vol. Vol. 5 No., pp. 52-58, 1996. 
[215] D. Yan, A. Khajepour, and R. Mansour, "Modeling of Two-Hot-Arm Horizontal Thermal Actuator," J. Micromech. Microeng., vol. Vol. 13, pp. 312-322, 2003.

[216] S. Timoshenko, "Analysis of bi-metal thermostats," J. Opt. Soc. Am, no. I, pp. 233-255, 1925.

[217] S. Scott and J. Kim, "An analytical capacitance model of temperature-sensitive, largedisplacement multimorph cantilevers: numerical and experimental validation," J. Microelectromechanical Syst., pp. 1-10, 2012.

[218] P. J. Gilgunn, N. Sarkar, and G. K. Fedder, "CMOS-MEMS Lateral Electrothermal Actuators," J. Microelectromechanical Syst., vol. 17, no. 1, pp. 103-114, Feb. 2008.

[219] S. T. Todd, S. Member, H. Xie, and S. Member, "An Electrothermomechanical Lumped Element Model of an Electrothermal Bimorph Actuator," pp. 1-13, 2007.

[220] J. W. Weaver, S. P. Timoshenko, and D. H. Young, Vibration Problems in Engineering. John Wiley \& Sons, 1990.

[221] E. B. Magrab, Vibrations of Elastic Systems: With Applications to MEMS and NEMS. Springer Netherlands, 2012.

[222] S. Han, H. Benaroya, and T. Wei, "Dynamics of transversely vibrating beams using four engineering theories," J. Sound Vib., vol. 225, pp. 935-988, 1999. 